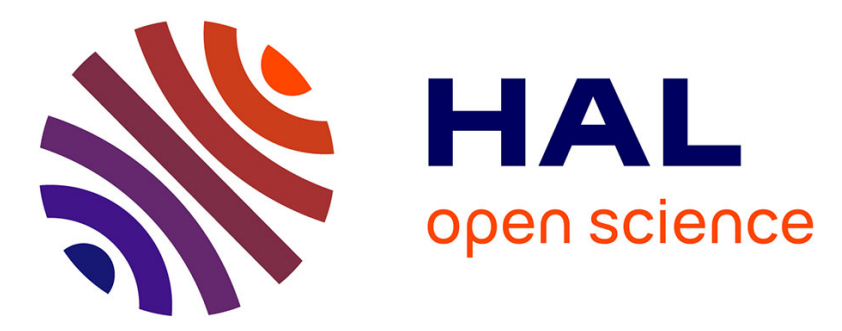

\title{
Trifluoromethyl Radical Triggered Radical Cyclization of N-Benzoyl Ynamides leading to Isoindolinones
}

Maud Cassé, Christian Nisole, Héloïse Dossmann, Yves Gimbert, Jean-Marie Fourquez, Laure Haberkorn, Ollivier Cyril, Fensterbank Louis

\section{- To cite this version:}

Maud Cassé, Christian Nisole, Héloïse Dossmann, Yves Gimbert, Jean-Marie Fourquez, et al.. Trifluoromethyl Radical Triggered Radical Cyclization of N-Benzoyl Ynamides leading to Isoindolinones. Science China Chemistry, 2019, 62, pp.1542-1546. 10.1007/s11426-019-9627-x . hal-02616094

\section{HAL Id: hal-02616094 \\ https://hal.science/hal-02616094}

Submitted on 24 May 2020

HAL is a multi-disciplinary open access archive for the deposit and dissemination of scientific research documents, whether they are published or not. The documents may come from teaching and research institutions in France or abroad, or from public or private research centers.
L'archive ouverte pluridisciplinaire HAL, est destinée au dépôt et à la diffusion de documents scientifiques de niveau recherche, publiés ou non, émanant des établissements d'enseignement et de recherche français ou étrangers, des laboratoires publics ou privés. 


\section{畛 SCIENCE CHINA \\ Chemistry}

\section{Trifluoromethyl Radical Triggered Radical Cyclization of N-Benzoyl Ynamides leading to Isoindolinones}

Fensterbank Louis, Casse Maud, Christian Nisole, Dossmann Heloise, Gimbert Yves, Fourquez Jean-Marie, Haberkorn Laure and Ollivier Cyril Citation: SCIENCE CHINA Chemistry; doi: 10.1007/s11426-019-9627-x

View online: http://engine.scichina.com/doi/10.1007/s11426-019-9627-x

Published by the Science China Press

Articles you may be interested in

A MASS SPECTROMETRIC STUDY ON THE RADICAL-INDUCED AROMATIC SUBSTITUTION BETWEEN BENZOYL PEROXIDE AND BENZENEDIOLS

Chinese Science Bulletin 37, 1891 (1992);

Trifluoromethylation recations through trifluoromethyl radical

SCIENTIA SINICA Chimica 42, 1417 (2012);

Tandem dual $\mathrm{C}\left(\mathrm{sp}^{2}\right)-\mathrm{H} / \mathrm{C}\left(\mathrm{sp}^{2} \underline{2}\right)-\mathrm{H}$ functionalization: a radical cyclization of 2-isocyanobiphenyl with ether to 6 -alkylated phenanthridine SCIENCE CHINA Chemistry 57, 1172 (2014);

ESR STUDY ON GENERATION OF N-ALKYL-p-PHENYLENEDIAMINE RADICAL CATIONS BY ULTRASOUND

Chinese Science Bulletin 35, 25 (1990);

Recent Advances in Radical-Mediated [2+2+m] Annulation of 1,n-Enynes

SCIENCE CHINA Chemistry 


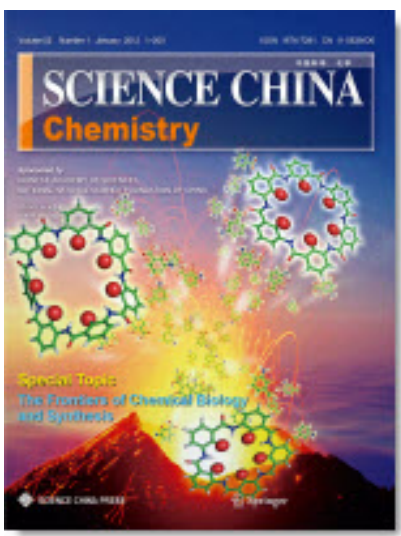

\section{Trifluoromethyl Radical Triggered Radical Cyclization of $\mathbf{N}$ - Benzoyl Ynamides leading to Isoindolinones}

\begin{tabular}{|c|c|}
\hline Journal: & SCIENCE CHINA Chemistry \\
\hline Manuscript ID & SCC-2019-0633.R1 \\
\hline Manuscript Type: & Communication \\
\hline $\begin{array}{r}\text { Date Submitted by the } \\
\text { Author: }\end{array}$ & 24-Sep-2019 \\
\hline Complete List of Authors: & $\begin{array}{l}\text { Fensterbank, Louis } \\
\text { Casse, Maud } \\
\text { Christian, Nisole } \\
\text { Dossmann, Heloise; Sorbonne Université } \\
\text { Gimbert, Yves ; Université Joseph Fourier } \\
\text { Fourquez, Jean-Marie } \\
\text { Haberkorn, Laure } \\
\text { Ollivier, Cyril; Sorbonne Université }\end{array}$ \\
\hline Keywords: & radical, photoredox chemistry, ynamide \\
\hline Speciality: & Organic Chemistry \\
\hline
\end{tabular}

\section{SCHOLARONE ${ }^{m}$ \\ Manuscripts}




\title{
Trifluoromethyl Radical Triggered Radical Cyclization of N-Benzoyl Ynamides leading to Isoindolinones
}

\author{
Maud Cassé ${ }^{1,2}$, Christian Nisole 2, Héloïse Dossmann ${ }^{1}$, Yves Gimbert ${ }^{1,3}$, Jean-Marie \\ Fourquez ${ }^{2}$, Laure Haberkorn ${ }^{2}$, Cyril Ollivier ${ }^{1^{*}}$ \& Louis Fensterbank ${ }^{1 *}$ \\ ${ }^{1}$ Sorbonne Université, CNRS, Institut Parisien de Chimie Moléculaire, IPCM, 75005 Paris, France \\ ${ }^{2}$ Institut de Recherches Servier, 78290 Croissy-sur-Seine, France \\ ${ }^{3}$ Département de Chimie Moléculaire, CNRS, Université Grenoble Alpes, 38610 Gières, France
}

Received .....; accepted ......; published online ......

\begin{abstract}
Under photocatalytic reductive conditions, trifluoromethyl radical addition onto an ynamide followed by cyclization on a benzoyl moiety produces diverse isoindolinone platforms with good yields. The selectivity of the radical cyclization, N-benzoyl vs. N-benzyl as radical acceptor and the $\mathrm{E} / \mathrm{Z}$ ratio of isomers have been rationalized by modeling.
\end{abstract}

Ynamides, trifluoromethylation, photocatalysis, cascade reactions, tandem processes, isoindolinones, modeling

\section{Introduction}

Inter- and intramolecular radical addition - cyclization tandems constitute a powerful method to assemble various scaffolds depending on the used partners [1-9]. For instance, this approach has been applied by using a trifluoromethyl radical as a trigger [10-12] to synthesize valuable fluorine containing substrates [13-16]. Because they have already proved to be versatile acceptors of electrophilic radicals like thiyl radicals (Scheme 1.1) [17-18], ynamides flanked with a

\footnotetext{
*Corresponding authors: cyril.ollivier@sorbonne-universite.fr louis.fensterbank@sorbonne-universite.fr

๑) Science China Press and Springer-Verlag Berlin Heidelberg 2015
}

pending aryl group are particularly attractive as partners since they would allow a direct access to isoindolinones bearing a vinyltrifluoromethyl moiety (Scheme 1.3). Benzoyl ynamides have previously been used as radical acceptors in cascades leading to the formation of isoindoles, isoindolinones, and pyridoisoindolones (Scheme 1.2) [19-21]. Very recently, this chemistry has been nicely extended by Evano and coworkers by using copper(I) photoredox catalysis (Scheme 1.2) [22]. These comforting reports set the ground for our project and among the specifications of the aimed synthesis, was the use of photoredox catalysis for the generation of the radical species which 
allows for sustainability as well as mild conditions to ensure molecular diversity [23-29]. We focused on generic precursors consisting of N-benzoyl ynamides. After intermolecular addition of the $\mathrm{CF}_{3}$ radical, the resulting vinyl radical $\mathbf{A}$ would undergo intramolecular addition on the pendant aromatic ring. Rearomatization involving the photocatalytic cycle would terminate the synthetic sequence and produce the isoindolinone products (Scheme 1.3).

Previous works

1) intermolecular addition of an electrophilic radical onto an ynamide [12]

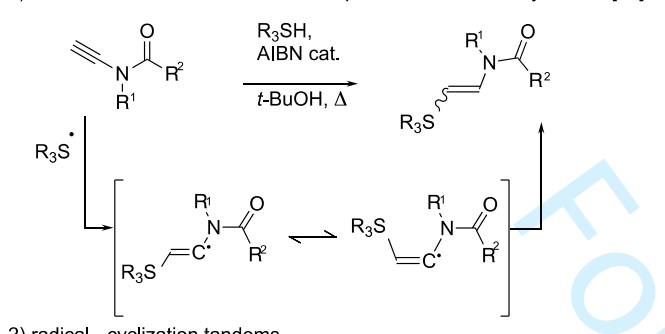

2) radical - cyclization tandems

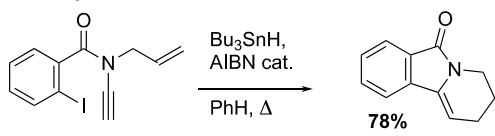

[14]

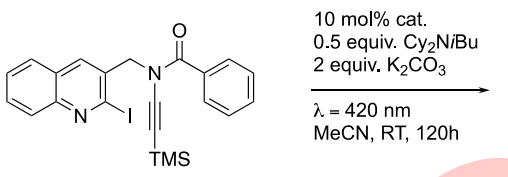

cat. $=[(\mathrm{DPE}(\mathrm{Phos})(\mathrm{bcp}) \mathrm{Cu}] \mathrm{PF}$

This work

3) intermolecular addition of $\mathrm{CF}_{3}$ radical - cyclization tandem in photoredox catalyzed conditions

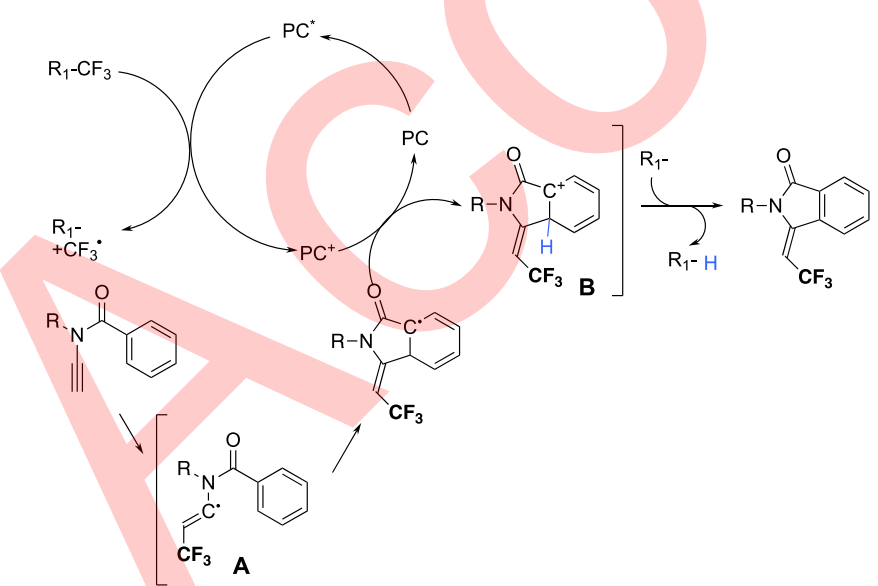

Scheme 1 Ynamides as radical acceptors: context and objective of the work

\section{Results and discussion}

To study the scope of this transformation, several terminal ynamides were prepared through Hsung's approach and conditions [30-32]. Copper-catalyzed alkynylation of the corresponding amides $\mathbf{1}$ using TIPS protected acetylene showed the best versatility with different tested substrates. Thus when using a combination of $\mathrm{CuSO}_{4} .5 \mathrm{H}_{2} \mathrm{O}, 1,10$-phenanthroline and a weak base $\left(\mathrm{K}_{3} \mathrm{PO}_{4}\right)$, no degradation was observed, despite long reaction times and the expected protected ynamides $\mathbf{2}$ were isolated in moderate to correct yields. As a representative example shown (Scheme 2), terminal ynamide 3a was obtained in $51 \%$ yield (2 steps) after deprotection of $\mathbf{2 a}$ with TBAF (Scheme 2). This synthetic pathway was applied for all the ynamide precursors 3a-q (see Supporting Information for details).

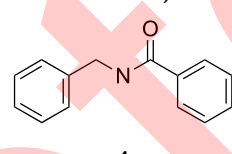

1a
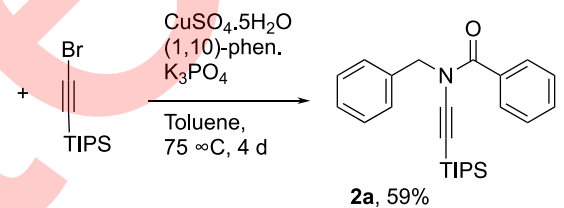

2a, $59 \%$ $\downarrow \begin{aligned} & \text { TBAF } \\ & \text { THF, } 0^{\circ} \mathrm{C}, 1 \mathrm{~h}\end{aligned}$

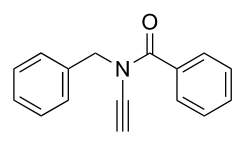

3a, $87 \%$

Scheme 2 Synthesis route for the ynamide $\mathbf{3 a}$

Our preliminary attempts of radical cyclization focused on the simplest term 3a with no substitution on the aromatic rings. Several parameters were examined such as the solvent, the source of trifluoromethyl radical and the photocatalyst (see Table 1). Acetonitrile, a traditional solvent in photoredox reaction conditions was first used as well as Togni II reagent as a trifluoromethyl radical source $[33,34]$ and we varied the photocatalyst keeping in mind the reduction potential of Togni II reagent $(-0,32$ $\mathrm{V} / \mathrm{SCE}$ in MeCN) [35]. While the use of $\mathrm{Ru}(\mathrm{bpy})_{3} \mathrm{Cl}_{2}$ (E $\mathrm{Ru}(\mathrm{II})^{*} / \mathrm{Ru}(\mathrm{III})=-0.81 \mathrm{~V} / \mathrm{SCE}$ in $\mathrm{MeCN}$ ) [36] validated the strategy, a slightly improved yield was obtained with the more reducing $\operatorname{Ir}(\mathrm{ppy})_{2}\left(\mathrm{~d}\right.$ tbpy). $\mathrm{PF}_{6} \quad(\mathrm{E}$ $\operatorname{Ir}(\mathrm{III})^{*} / \operatorname{Ir}(\mathrm{IV})=-0.96 \mathrm{~V} / \mathrm{SCE}$ in $\left.\mathrm{MeCN}\right)$. A trend which was confirmed with $\operatorname{Ir}(\text { ppy })_{3}\left(\mathrm{E} \operatorname{Ir}(\mathrm{III})^{*} / \operatorname{Ir}(\mathrm{IV})=\right.$ $1.73 \mathrm{~V} / \mathrm{SCE}$ in $\mathrm{MeCN}$ ) since the best yield of $4 \mathbf{a}$ was obtained $(71 \%)$. While rarely used in photoreductive processes [37-40], 4CzIPN could provide some interesting reactivity but in a weaker yield (50 \% of 4 a). 
We nevertheless studied other ynamide precursors 3 and obtained cyclization products 4 (Scheme 4) in moderate to good yields. The N-group was substituted by a p-methoxybenzyl (PMB) and a 2,4-dimethoxybenzyl protecting groups (4b and $4 c$ ) without trouble. Finally, the $\mathrm{N}$-methyl substituted isoindolinone $4 \mathrm{~d}$ could also be obtained in $47 \%$. We also varied the aryl group of the benzoyl moiety. The transformation was compatible with a broad range of substituents on para position such as alkyles (4e, 4i), halogens $(4 \mathbf{f}, \mathbf{4 g}, 4 \mathrm{~h})$ or other electron-withdrawing groups (nitro $\mathbf{4} \mathbf{j}$, cyano $\mathbf{4 k}$, ester $\mathbf{4 l}$ ) leading to cascade adducts in moderate to good yields $(40-61 \%)$. Pleasingly, a pyridyl moiety was amenable to this tandem process giving rise to an original heterocyclic framework (54\% of $4 \mathrm{~m}$ ). With ortho-substituted benzoyl moieties, the reaction proved to be more sluggish $(24 \%$ of $4 \mathbf{p})$ but only one regioisomer was isolated, resulting from the homolytic aromatic substitution at the non-substituted position [44].

It was also observed that the cyclisation process was favored compared to a $1.5-H$ shift on an alkyl chain $(57 \%$ of $\mathbf{4 q})$ [45].

Scheme 4 Scope of the process (E/Z ratio precised) 

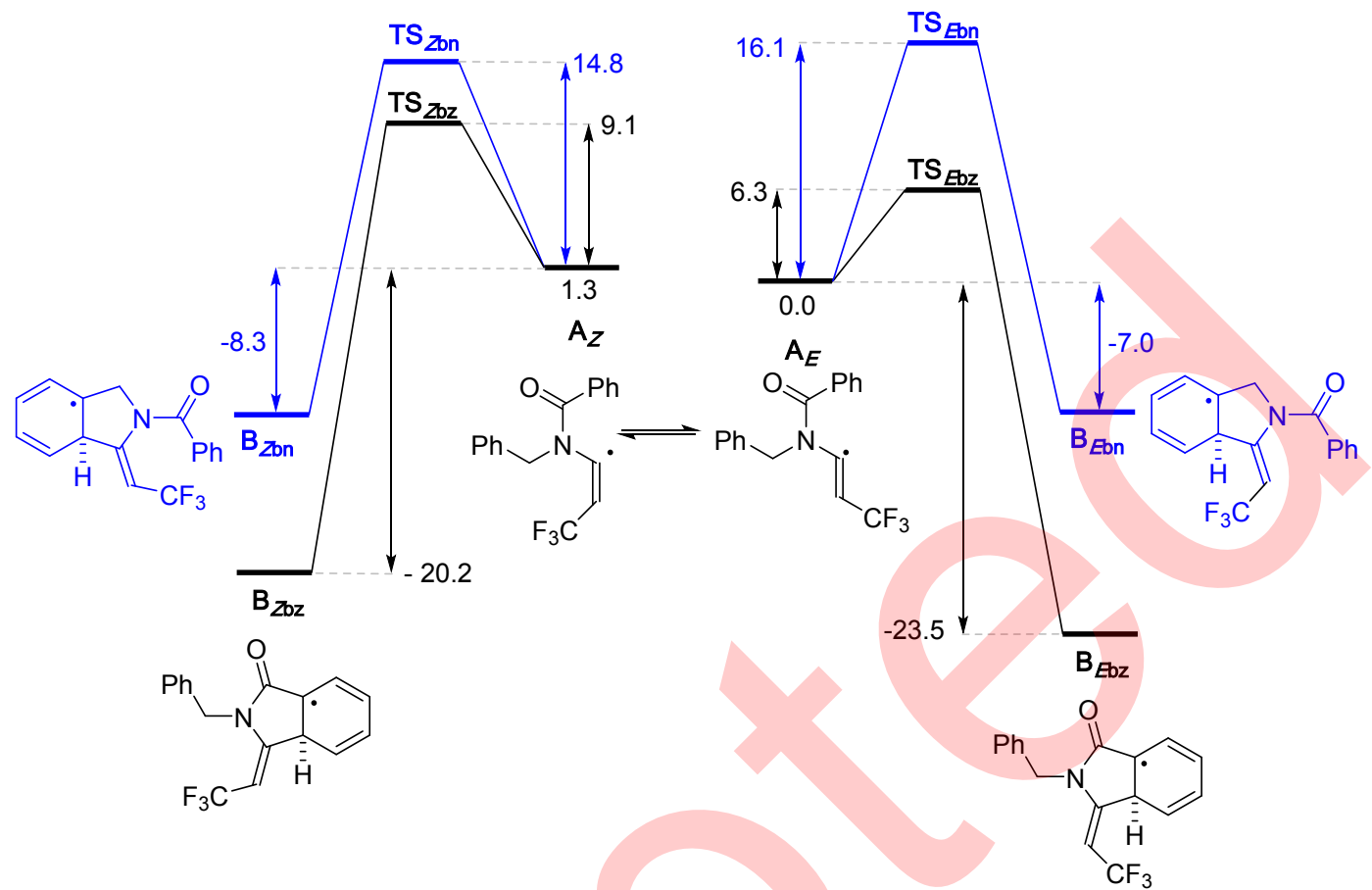

Scheme 5 Energetic profiles for cyclisation of ynamides: energies given are $\Delta \mathrm{G}$ in $\mathrm{kcal} / \mathrm{mol}$.

All these findings confirmed the validity of this approach to synthesize isoindolinones. It was also notable that the cyclization of the intermediate vinyl radical always took place on the $\mathrm{N}$-benzoyl moiety and no product resulting from the cyclization on the benzyl group was isolated. To rationalize this observation, electronic structure calculations were performed, using the PBE0-D3BJ/def2TZVP level (Scheme 5). The addition of the $\mathrm{CF}_{3}$ radical on the ynamide happens to be a very exothermic barrier-free process $(\Delta \mathrm{G}=-33.7$ $\mathrm{kcal} / \mathrm{mol}$, see SI).The $\mathrm{E}$ or $\mathrm{Z}$ configuration of the vinyl radical does not seem to influence the stability of the species, both indeed differ only by $1.3 \mathrm{kcal} / \mathrm{mol}$. From the vinyl radical $A_{E}$, the intramolecular cyclization towards the benzoyl moiety proceeds via a barrier of $6.3 \mathrm{kcal} / \mathrm{mol}$ whereas the addition of the radical on the benzyl side costs $16.1 \mathrm{kcal} / \mathrm{mol}$ (black and blue pathways, Scheme 5). The same observation was made for the vinyl radical of $\mathrm{Z}$ configuration although the difference between the barriers of the two paths is smaller (ca. 6 vs $10 \mathrm{kcal} / \mathrm{mol}$ for the E variant). Finally, cyclohexadienyl radicals of type $\mathbf{B}$, obtained after cyclization, are again much more stable than the cyclohexadienyl radicals resulting from the cyclization onto the benzyl group (by $16.5 \mathrm{kcal} / \mathrm{mol}$ for the $\mathrm{E}$ isomer and $11.9 \mathrm{kcal} / \mathrm{mol}$ for the $\mathrm{Z}$ isomer).
Considering more carefully the electronic structure and molecular orbitals (MOs) of the intermediate vinyl radical helps to shed more light on the unexpected regioselectivity of this cyclization process. The DFT-computed electronic structure of radical $\mathbf{A}$ is displayed on Figure 1 together with the Kohn-Sham orbitals. The SOMO orbital is localized mainly on the vinyl moiety, in accordance with the expected structure of this intermediate. Interestingly, the three first LUMO exhibit an electron density localized on the same region but also on the benzoyl moiety. This observation aligns with the experimental observation that the benzoyl moiety favours cyclisation. Therefore, the weaker LUMO-SOMO gap orients the radical attack on the benzoyl group only. 
September (2019) Vol. No.

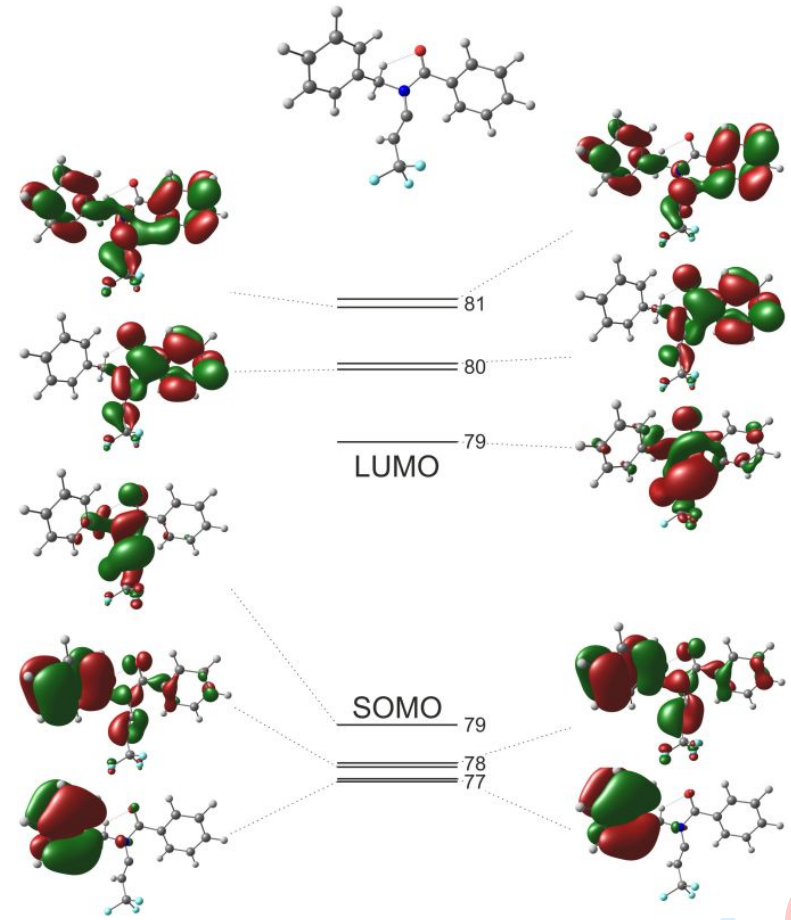

Figure 1 DFT-computed structure of $\mathbf{A}_{E}$ and corresponding Kohn-Sham orbitals.

\section{Conclusions}

In summary, we reported a new cascade reaction for the synthesis of trifluoromethyl substituted isoindolinones, involving sequential radical trifluoromethylation of ynamides followed by radical cyclization onto benzoyl moieties under photocatalytic conditions. The regioselectivity of the cyclization process (N-benzyl vs. N-benzoyl) is controlled and the $E$ isomer is formed as major diastereomer. These findings were supported by DFT calculations.

Acknowledgments We thank Sorbonne Université, CNRS and Servier for funding. The authors wish to acknowledge the analytical department of IDRS - Servier for the compounds analyses (IR, NMR, HR-MS) and the SRIMC department for the syntheses on big scale. This work was granted access to the high performance computing (HPC) resources of the HPCaVe Centre at Sorbonne Université and to the HPC resources of IDRIS under the allocation 2018-A0050810312 made by GENCI. The authors wish to acknowledge support from the ICMG Chemistry Nanobio Platform-PCECIC, Grenoble, for calculations facilities. Jérémy Forté is acknowledged for the X-ray diffraction analyses as well as Conor Dent Cullen for proofreading the manuscript.
Conflict of interest The authors declare that they have no conflict of interest.

Supporting information The supporting information is available online at http://chem.scichina.com and http://link.springer.com/journal/11426. The Y-Ray crystal structures were deposited at the Cambridge Crystallographic Data Centre with numbers CCDC 1949295 (compound 4aE) and CCDC 1949296 (compound $4 \mathrm{aZ}$ ) and can be obtained free of charge via www.ccdc.cam.ac.uk.

1 Albert M, Fensterbank L, Lacôte E, Malacria M. Topics Current Chemistry, 2006, 264, 1-62

2 Baralle A, Baroudi A, Daniel M, Fensterbank L, Goddard JP, Lacôte E, Larraufie MH., Maestri G, Malacria M, Ollivier C. Radical Cascade Reactions. In: Chatgilialoglu C, Studer A, Eds. Encyclopedia of Radicals in Chemistry, Biology and Materials. John Wiley \& Sons: Chichester, U. K., 2012, 729-766

3 Godineau E, Landais Y. Chem Eur J, 2009, 15 (13), 3044-3055

4 Liautard V, Landais Y, in:. Zhu J, Wang Q, Wang MX, Eds. Multicomponent Reactions. Wiley, 2nd Edition. 2014, 401-438

5 Chen JR, Yu XY, Xiao WJ, Synthesis, 2015, 47, 604-629

6 Zhang Y, Sun K, Lv Q, Chen X, Qu L, Yu B, Chin Chem Lett, 2019, 30, 1361-1368

7 Xuan J, Studer A, Chem Soc Rev, 2017, 46, 4329-4346

8 Huang HM, Garduno-Castro MH, Morrill C, Procter DJ, Chem Soc Rev, 2019, 48, 4626-4638

9 Dutta S, Mallick RK, Prasad R, Gandon V, Sahoo KA, Angew Chem Int Ed, 2019, 58, 2289-2294

10 Wang J, Sánchez-Roselló M, Acena JL, del Pozo C, Sorochinsky A E, Fustero S, Soloshonok VA, Liu H. Chem. Rev. 2014, 114, 2432-2506

11 Dagousset G, Carboni A, Masson G, Magnier E. Visible light-induced (per)fluoroalkylation by photoredox catalysis. In Modern Synthesis Process and Reactivity of Fluorinated Compounds: Progress in Fluorine Science; Elsevier, 2017, 389-426

12 Oh EH, Kim HJ, Han SB. Synthesis, 2018, 50, 3346-3358

13 Fuentes N, Kong W, Fernandez-Sanchez L, Merino E, Nevado C. J Am Chem Soc, 2015, 137, 964-973

14 Zheng J, Deng Z, Zhang Y, Cui S. Adv Synth Cat, 2016, 358, 746-751

15 Li Y, Lu Y, Qiu G, Ding Q. Org Lett, 2014, 16, 4240-4243

16 Noto N, Miyazawa K, Koike T, Akita M. Org Lett, 2015, 17, 3710-3713

17 Baneerjee B, Litvinov DN, Kang J, Bettale JD, Castle SL. Org Lett, 2010, 12, 2650-2652

18 Sato A, Yorimitsu H, Oshima K. Synlett, 2009, 28-31

19 Marion F, Courillon C, Malacria M. Org Lett, 2003, 5, 5095-5097

20 Marion F, Coulomb J, Servais A, Courillon C, Fensterbank L, Malacria M. Tetrahedron, 2006, 63, 3856-3871

21 Balieu S, Toutah K, Carro L, Chamoreau LM, Rousselière H, Courillon C. Tetrahedron Lett, 2011, 2876-2880

22 Baguia H, Deldaele C, Romero E, Michelet B, Evano G. Synthesis, 2018, 50, 3022-3030

23 Wang CS, Dixneuf PH, Soulé JF. Chem Rev, 2018, 118, 7532-7585

24 Staveness D, Bosque I, Stephenson CRJ. Acc Chem Res, 2016, 49, 
2295-2306

25 Shaw MH, Twilton J, MacMillan DWC. J Org Chem, 2016, 81, 6898-6926

26 Pawlowski R, Stanek F, Stodulski M, Molecules, 2019, 24, 1533-1566

27 Festa AA, Voskressensky LG, Van der Eycken EV, Chem Soc Rev, 2019, 48, 4401-4423

28 Chen JR, Hu XQ, Lu LQ, Xiao WJ, Acc Chem Res, 2016, 49, 1911-1923

29 Tanoury GJ, Synthesis, 2016, 48, 2009-2025

30 Cook AM, Wolf C. Tetrahedron Lett, 2015, 56, 2377-2392

31 Evano G, Coste A, Jouvin K. Angew Chem Int Ed, 2010, 49, 2840-2859

32 Zhang Y, Hsung RP, Tracey MR, Kurtz KCM, Vera EL. Org Lett, 2004, 6, 1151-1154

33 Charpentier J, Früh N, Togni A. Chem Rev, 2015, 115, 650-682

34 Eisenberger P, Gischig S, Togni A. Chem Eur J, 2006, 12, 2579-2586

35 For trifluoromethyl-containing compounds redox potentials: Jiang Y, Yu H, Fu Y, Liu L. Sci China Chem, 2015, 58, 673-683

36 For photocatalysts redox potentials: Prier CK, Rankic DA, MacMillan DWC. Chem Rev, 2013, 113, 5322-5363

37 For first uses in photoredox catalysis, see: Luo J, Zhang J. ACS Catal, 2016, 6, 873-877

38 For first uses in photoredox catalysis, see: Lévêque C, Chenneberg L, Corcé V, Ollivier C, Fensterbank L. Chem Comm, 2016, 52, 9877-9880

39 For a review, see: Shang TY, Lu LH, Cao Z, Liu Y, He WM, Yu B. Chem Commun, 2019, 55, 5408-5419

40 For a revision of the redox potentials, see: Vaillant FL, Garreau M, Nicolai S, Gryn'ova G, Corminboeuf C, Waser J. Chem Sci, 2018, 9, 5883-5889

41 Jacquet J, Blanchard S, Derat E, Desage El-Murr M, Fensterbank L. Chem Sci, 2016, 7, 2030-2036

42 Singh K, Staig SJ, Weaver JD. J Am Chem Soc, 2014, 136, 5275-5278

43 Lin QY, Xu XH, Qing FL. J Org Chem, 2014, 79, 10434-10446

44 Larraufie MH, Courillon C, Ollivier C, Lacôte E, Malacria M, Fensterbank L. J Am Chem Soc, 2010, 132, 4381-4387

45 Bogen S, Gulea M, Fensterbank L, Malacria M. J Org Chem, 1999, 64, 4920-4925

Table of Contents graphic<smiles>[R]N(C#C)C(=O)c1ccc([N+]([O-])([O-])[O-])cc1</smiles>

regio- and stereselective tandem
Authors are required to submit a graphic for the TOC that, in conjunction with the manuscript title, conveys to readers the essence of the work and thereby capture their attention. It may be a key structure, reaction, equation, concept, theorem, or an ingenious representation discussed in the manuscript. TOC graphic should have a size of no larger than $8 \mathrm{~cm} \times 6 \mathrm{~cm}$ and appear on the last page of the text file. 


\section{SUPPORTING INFORMATION}

\section{Trifluoromethyl Radical Triggered Radical Cyclization of N-Benzoyl Ynamides leading to Isoindolinones}

Maud Cassé ${ }^{1,2}$, Christian Nisole ${ }^{2}$, Héloïse Dossmann ', Yves Gimbert ${ }^{1,3}$, JeanMarie Fourquez ${ }^{2}$, Laure Haberkorn ${ }^{2}$, Cyril Ollivier ${ }^{10}$ \& Louis Fensterbank ${ }^{*}$

'Sorbonne Université, CNRS, Institut Parisien de Chimie Moléculaire, IPCM, 75005 Paris, France Institut de Recherches Servier, 78290 Croissy-sur-Seine, France

Département de Chimie Moléculaire, CNRS, Université Grenoble Alpes, 38610 Gières, France

E-mail : cyril.ollivier@sorbonne-universite.fr

louis.fensterbank@sorbonne-universite.fr

Table of contents

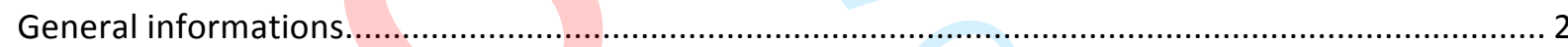

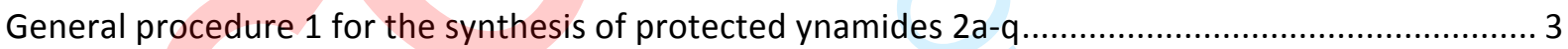

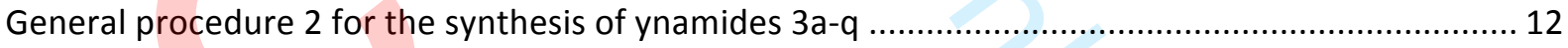

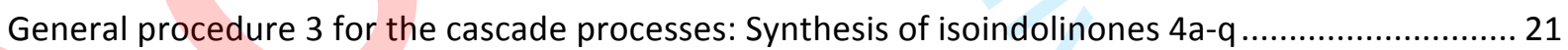

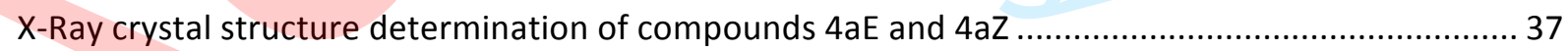

DFT calculation method, energetics data and cartesian coordinates ............................................... 41

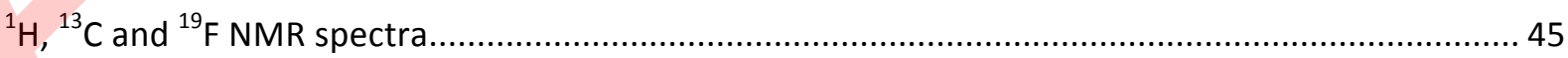




\section{General informations}

Photocatalyzed reactions were irradiated under a blue LED light array homemade apparatus. Reactions were carried out in Schlenk tubes at a distance of approximately $2 \mathrm{~cm}$ from the LEDs and an average temperature of $27^{\circ} \mathrm{C}$ was maintained by an air cooling system.
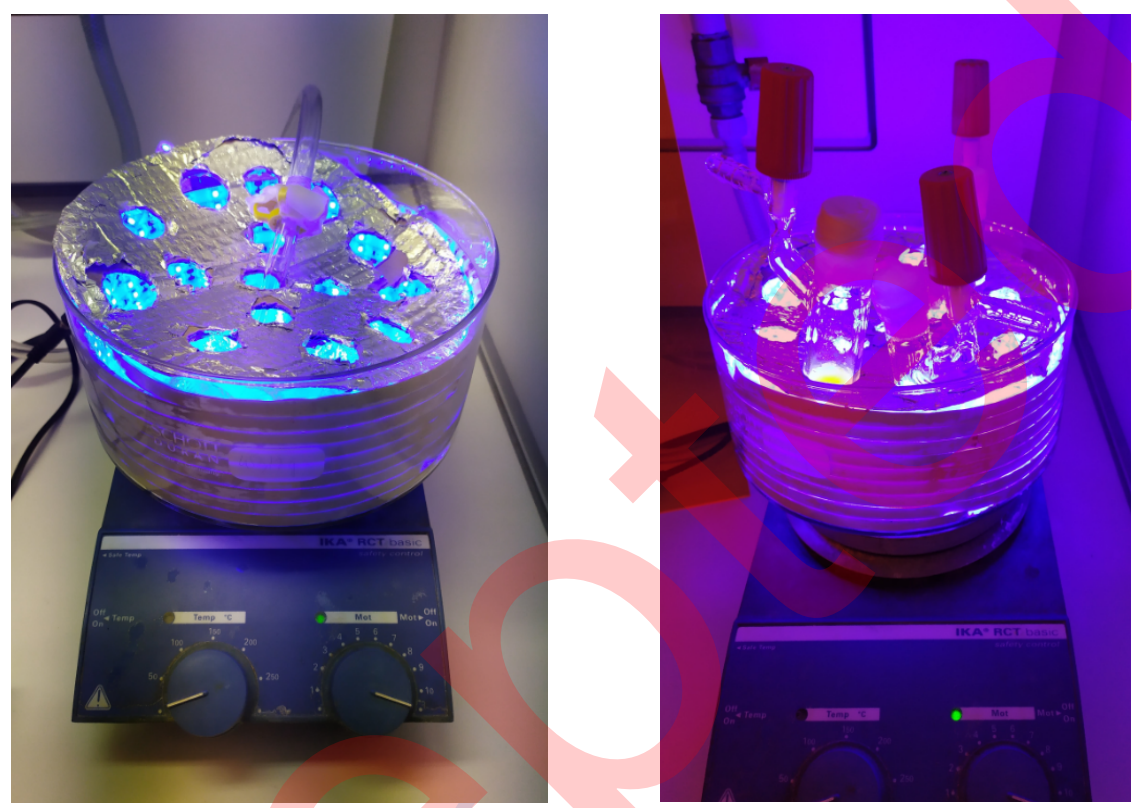

If there were issues with solubility (syntheses of compounds $\mathbf{4 j}$ and $\mathbf{4 k}$ ), reactions were heated at $40^{\circ} \mathrm{C}$ in an oil bath and irradiation occurred with a $365 \mathrm{~nm}$ Evoluchem ${ }^{\mathrm{TM}}$ LED.

All reactions were performed under an atmosphere of argon unless otherwise stated, and monitored by thin layer chromatography (T.L.C.) or UPLC-MS. T.L.C. were carried out on ready for use plates of silica gel 60 F254 (Merck) and visualization was performed under an U.V. lamp at $254 \mathrm{~nm}$. Reaction monitoring was conducted with Waters Acquity ${ }^{\circledR}$ instrument, fitted with PDA and SQD2 detectors (ESI source in positive mode). Injection volumes were 0.5 or $1 \mu \mathrm{L}$, the column was a Waters Acquity UPLC ${ }^{\circledast} \quad \mathrm{BEH}^{\mathrm{C}} \mathrm{C}_{18} 1.7 \mu \mathrm{m}$ and temperature was kept at $40^{\circ} \mathrm{C}$. Mobile phase A consisted of $\mathrm{H}_{2} \mathrm{O} / \mathrm{MeCN} /$ formic acid (98/2/0.1) and mobile phase B of MeCN/ $\mathrm{H}_{2} \mathrm{O} /$ formic acid (98/2/0.1). Gradient ran from $100 \%$ A to $100 \%$ B in 3 min. Products were purified by flash chromatography using packed silica gel columns. Unless otherwise specified, all commercially available reagents were used as received.

All the analyses were conducted by Servier analysis department. ${ }^{1} \mathrm{H}$ and ${ }^{13} \mathrm{C}$ NMR spectra were recorded on Brüker 400 or $500 \mathrm{MHz}$ machine operating at ambient temperature probe, in DMSO- $d_{6}$ or $\mathrm{CDCl}_{3} .{ }^{1} \mathrm{H}$ NMR spectra were recorded at $400 \mathrm{MHz}$ or $500 \mathrm{MHz},{ }^{13} \mathrm{C} \mathrm{NMR}$ spectra were recorded at $75 \mathrm{MHz}$ or $100 \mathrm{MHz}$ and ${ }^{19} \mathrm{~F}$ spectra were recorded at $376 \mathrm{MHz}$ on a Brüker $400 \mathrm{M} \mathrm{Hz}$ machine

Chemical shifts $(\delta)$ were quoted in parts per million (ppm). For ${ }^{1} \mathrm{H}$ NMR spectra, the chemical shifts were attributed relative to residual $\mathrm{CHCl}_{3}(7.26 \mathrm{ppm})$ or residual DMSO $(2.50 \mathrm{ppm})$. For ${ }^{13} \mathrm{C} \mathrm{NMR}$ spectra (JMOD), the attributions were done relative to residual $\mathrm{CHCl}_{3}(77.16 \mathrm{ppm}$ ) or residual DMSO (40 ppm). Coupling constants $(J)$ were quoted in Hertz $(\mathrm{Hz})$. The following abbreviations were used to give the multiplicity of the NMR signals: s: singlet, bs: broad singlet, d: doublet, t: triplet, q: quartet, quint: quintet, dd: doublet - doublet... Infrared spectra were recorded with the ATR technic on a 
Brüker Tensor 27 spectrometer. Mass spectrometry (HRMS) was performed with a Thermo-Fischer Scientific spectrometer (by ESI+: Orbitrap Velos Pro or by EI: HREIGC, HRMS magnetic Sector Ms DFS). In a few cases, the mass spectrometry was done on an Agilent Technologies (ESI; LC/MSD TOF). Melting points were measured on the Büchi Melting point B-540 apparatus.

\section{General procedure 1 for the synthesis of protected ynamides 2a-q}

Amides 1a-q were synthesized according to the literature. ${ }^{1}$

In a sealed tube (Radley) was loaded the amide 1 ( 1 equiv.), $\mathrm{CuSO}_{4} .5 \mathrm{H}_{2} \mathrm{O}$ (0.2 equiv.), 1,10phenanthroline ( 0.4 equiv.) and $\mathrm{K}_{3} \mathrm{PO}_{4}$ (2.2 equiv.) under argon. To this mixture was added the 2bromoethynyl(triisopropyl)silane ${ }^{2,3}(1.1$ equiv., $0.1 \mathrm{M})$ in toluene. The reaction mixture was capped and heated at $75^{\circ} \mathrm{C}$ for a given reaction time. The reaction mixture was cooled down to room temperature, filtrated through Celite (rinced with DCM). The filtrate was concentrated under vacuum. The crude product was purified by silica gel flash column chromatography (liquid deposit in Heptane/ $\mathrm{CH}_{2} \mathrm{Cl}_{2}$ 50/50; gradient eluent, $\mathrm{CH}_{2} \mathrm{Cl}_{2}$ in heptane/pentane or diethyl ether in pentane).

General procedure 1': use of $\mathrm{CuSO}_{4} .5 \mathrm{H}_{2} \mathrm{O}$ (0.1 equiv.), 1,10-phenanthroline (0.2 equiv.), $\mathrm{K}_{3} \mathrm{PO}_{4}$ (2 equiv.) and 2-bromoethynyl(triisopropyl)silane (1.2 equiv., $0.1 \mathrm{M})$.

\section{N-benzyl-N-(2-triisopropylsilylethynyl)benzamide 2a}<smiles>CC(C)C(C)(C)[Si](C#CCN(Cc1ccccc1)C(=O)c1ccccc1)(C(C)C)C(C)C</smiles>

Starting from N-benzylbenzamide $1 \mathrm{a}(2.83 \mathrm{~g}, 13.5 \mathrm{mmol}), \mathrm{CuSO}_{4} .5 \mathrm{H}_{2} \mathrm{O}(335 \mathrm{mg}, 1.4 \mathrm{mmol}), 1,10-$ phenanthroline $(485 \mathrm{mg}, \quad 2.7 \mathrm{mmol}), \mathrm{K}_{3} \mathrm{PO}_{4} \quad\left(\begin{array}{lllll}5.71 & \mathrm{~g}, & 26.9 \mathrm{mmol}) & \text { and 2- }\end{array}\right.$ bromoethynyl(triisopropyl)silane $(4.21 \mathrm{~g}, 16.0 \mathrm{mmol})$ under argon, using General procedure $\mathbf{1}^{\prime}$ and heating at $75^{\circ} \mathrm{C}$ for 3.5 days. The crude was purified to afford N-benzyl-N-(2triisopropylsilylethynyl)benzamide $2 \mathrm{a}$ as a yellow oil $(3.10 \mathrm{~g}, 59 \%$ yield). Spectral data are in accordance with those reported in the literature. ${ }^{4}$

IR (neat): $2167,1676,881,745 \mathrm{~cm}^{-1}$

${ }^{1} \mathbf{H}$ NMR $\left(400 \mathrm{MHz}\right.$, DMSO-d $\left.d_{6}\right) \delta \mathrm{ppm} 7.7(\mathrm{~d}, J=7.7 \mathrm{~Hz}, 2 \mathrm{H}), 7.55(\mathrm{tt}, J=7.5,1.5 \mathrm{~Hz}, 1 \mathrm{H}), 7.45(\mathrm{t}, J=$ $7.6 \mathrm{~Hz}, 2 \mathrm{H}), 7.4(\mathrm{~m}, 5 \mathrm{H}), 4.85(\mathrm{~s}, 2 \mathrm{H}), 0.8(\mathrm{~s}, 21 \mathrm{H})$

\footnotetext{
${ }^{1}$ For the synthesis of the amides, see: Couty S, Liegault B, Meyer C, Cossy J. Tetrahedron 2006, 62, 3882-3895

${ }^{2}$ Nicolai S, Sedigh-Zadeh R, Waser J. J. Org. Chem. 2013, 78, 3783-3801

${ }^{3}$ Rubin Y, Lin SS, Knobler CB, Anthony J, Boldi AM, Diederich F. J. Am. Chem. Soc. 1991, 113, 6943-6949

${ }^{4}$ Zhang X, Zhang Y, Huang J, Hsung RP, Tracey MR, Kurtz KCM, Oppenheimer J, Petersen ME, Sagamanova IK, Shen L, Tracey MR. J. Org. Chem. 2006, 71, 4170-4177
} 
${ }^{13} \mathrm{C}$ NMR $\left(125 \mathrm{MHz}, \mathrm{DMSO}-d_{6}\right) \delta \mathrm{ppm} 170.6,136.2,133.8,131.7,128.9(2 \mathrm{C}), 128.8(2 \mathrm{C}), 128.6(2 \mathrm{C})$, $128.5(2 \mathrm{C}), 128.4,99.4,71.5,52.3,18.7(6 \mathrm{C}), 11(3 \mathrm{C})$

\section{$\mathrm{N}$-[(4-methoxyphenyl)methyl]-N-(2-triisopropylsilylethynyl)benzamide 2b}<smiles>COc1ccc(CN(C#CN(C(=O)c2ccccc2)[Si](C(C)C)(C(C)C)C(C)C)C(C)C)cc1</smiles>

Starting from N-[(4-methoxyphenyl)methyl]benzamide $\mathbf{1 b}(6.04 \mathrm{~g}, 25 \mathrm{mmol}), \mathrm{CuSO}_{4} .5 \mathrm{H}_{2} \mathrm{O}(445 \mathrm{mg}$, $2.5 \mathrm{mmol}), 1,10$-phenanthroline $(902 \mathrm{mg}, 5.0 \mathrm{mmol}), \mathrm{K}_{3} \mathrm{PO}_{4}(6.71 \mathrm{~g}, 50.1 \mathrm{mmol})$ and 2bromoethynyl(triisopropyl)silane $(7.85 \mathrm{~g}, 30.0 \mathrm{mmol})$ under argon, using General procedure $\mathbf{1}^{\prime}$ and heating at $75^{\circ} \mathrm{C}$ for 4.5 days. The crude was purified to afford $\mathrm{N}$-[(4-methoxyphenyl)methyl]- $\mathrm{N}-(2-$ triisopropylsilylethynyl)benzamide $\mathbf{2 b}$ as a yellow oil (1.87 g, $18 \%$ yield).

IR (neat): $2171,1678 \mathrm{~cm}^{-1}$

${ }^{1} \mathbf{H}$ NMR $\left(500 \mathrm{MHz}\right.$, DMSO- $\left.d_{6}\right) \delta \mathrm{ppm} 7.69(\mathrm{~d}, J=7.5 \mathrm{~Hz}, 2 \mathrm{H}), 7.52(\mathrm{t}, J=7.5 \mathrm{~Hz}, 1 \mathrm{H}), 7.43(\mathrm{t}, J=7.5$ $\mathrm{Hz}, 2 \mathrm{H}), 7.32(\mathrm{~d}, J=8.0 \mathrm{~Hz}, 2 \mathrm{H}), 6.92(\mathrm{~d}, J=8.0 \mathrm{~Hz}, 2 \mathrm{H}), 4.75(\mathrm{~s}, 2 \mathrm{H}), 3.74(\mathrm{~s}, 3 \mathrm{H}), 0.82(\mathrm{~s}, 18 \mathrm{H}), 0.82$ $(\mathrm{s}, 3 \mathrm{H})$

${ }^{13} \mathrm{C}$ NMR (125 MHz, DMSO- $\left.d_{6}\right) \delta \mathrm{ppm} 170.9,160,134.2,132.2,130.5(2 \mathrm{C}), 128.5(2 \mathrm{C}), 128.3(2 \mathrm{C})$, $128.1,114.3(2 \mathrm{C}), 100,71.6,55.6,51.9,18.8(6 \mathrm{C}), 11.1$ (3C)

HRMS (ESI): calcd. for $\mathrm{C}_{26} \mathrm{H}_{36} \mathrm{~N} \mathrm{O}$ Si $\left([\mathrm{M}+\mathrm{H}]^{+}\right) 422.2510$, found 422.2511

\section{$\mathrm{N}$-[(2,4-dimethoxyphenyl)methyl]-N-(2-triisopropylsilylethynyl)benzamide 2c}<smiles>COc1ccc(CN(C#CC(C)(C(C)C)C(C)C)C(=O)c2ccccc2)c(OC)c1</smiles>

Starting from N-[(2,4-dimethoxyphenyl)methyl]benzamide 1c $(940 \mathrm{mg}, 3 \mathrm{mmol})$, Cul $(57 \mathrm{mg}, 0.3$ mmol), 1,10-phenanthroline (108 $\mathrm{mg}, 0.6 \mathrm{mmol}), \mathrm{K}_{3} \mathrm{PO}_{4}(1.27 \mathrm{~g}, 6 \mathrm{mmol})$ and 2bromoethynyl(triisopropyl)silane (941 $\mathrm{mg}, 3.6 \mathrm{mmol}$ ) under argon, using General procedure $\mathbf{1}^{\prime}$ and heating at $75^{\circ} \mathrm{C}$ for 4 days. The crude was purified to afford $\mathrm{N}-[(2,4-$ dimethoxyphenyl)methyl]-N-(2triisopropylsilylethynyl)benzamide $\mathbf{2 c}$ as a yellow oil ( $423 \mathrm{mg}, 30 \%$ yield).

IR (neat): 2170, 1681, 1612, 1591, $1512 \mathrm{~cm}^{-1}$

${ }^{1} \mathrm{H}$ NMR $\left(500 \mathrm{MHz}, \mathrm{DMSO}-d_{6}\right) \delta \mathrm{ppm} 7.65(\mathrm{dd}, J=7.6,1.4 \mathrm{~Hz}, 2 \mathrm{H}), 7.5(\mathrm{tt}, J=7.4,1.3 \mathrm{~Hz}, 1 \mathrm{H}), 7.42(\mathrm{t}$, $J=7.5 \mathrm{~Hz}, 2 \mathrm{H}), 7.2(\mathrm{~d}, J=8.4 \mathrm{~Hz}, 1 \mathrm{H}), 6.58(\mathrm{~d}, J=2.4 \mathrm{~Hz}, 1 \mathrm{H}), 6.49(\mathrm{dd}, J=8.3,2.4 \mathrm{~Hz}, 1 \mathrm{H}), 4.71(\mathrm{~s}, 2$ H), $3.77 / 3.75(2 \mathrm{~s}, 6 \mathrm{H}), 0.76(\mathrm{~d}, 18 \mathrm{H}), 0.75(\mathrm{~m}, 3 \mathrm{H})$ 
${ }^{13} \mathrm{C}$ NMR $\left(125 \mathrm{MHz}\right.$, DMSO- $\left.d_{6}\right) \delta \mathrm{ppm} 170.7,161.3,159,134.4,131.8,131.5,128.5(2 \mathrm{C}), 128.4(2 \mathrm{C})$, $115.6,105.01,99.3,98.8,71.3,56,55.7,47.1,18.7$ (6C), 11.02 (3C)

HRMS (ESI): calcd. for $\mathrm{C}_{27} \mathrm{H}_{37} \mathrm{NO}_{3} \mathrm{Si}\left([\mathrm{M}]^{+}\right)$451.2537, found 451.2518

\section{N-methyl-N-(2-triisopropylsilylethynyl)benzamide 2d}<smiles>CC(C)[Si](C#CN(C)C(=O)c1ccccc1)(C(C)C)C(C)C</smiles>

Starting from N-methyl-N-(2-triisopropylsilylethynyl)benzamide $1 \mathrm{~d}(700 \mathrm{mg}, 5.18 \mathrm{mmol}), \mathrm{CuSO}_{4} .5 \mathrm{H}_{2} \mathrm{O}$ (129 mg, $0.52 \mathrm{mmol}), 1,10$-phenanthroline (187 mg, $1.0 \mathrm{mmol}), \mathrm{K}_{3} \mathrm{PO}_{4}(2.20 \mathrm{~g}, 10.4 \mathrm{mmol})$ and 2bromoethynyl(triisopropyl)silane $(1.49 \mathrm{~g}, 5.7 \mathrm{mmol}, 1.1$ equiv.) under argon, using General procedure $1^{\prime}$ and heating at $75^{\circ} \mathrm{C}$ for 4 days. The crude was purified to afford N-methyl- $\mathrm{N}-(2-$ triisopropylsilylethynyl)benzamide $\mathbf{2} \mathbf{d}$ as a yellow oil $(682 \mathrm{mg}, 42 \%$ yield). Spectral data are in accordance with those reported in the literature ${ }^{4}$.

IR (neat): $2167,1680,1603,1580 \mathrm{~cm}^{-1}$

${ }^{1} \mathbf{H}$ NMR $\left(400 \mathrm{MHz}, \mathrm{CDCl}_{3}\right) \delta \mathrm{ppm}$ 7.81-7.76 (m, $\left.2 \mathrm{H}\right), 7.48-7.41(\mathrm{~m}, 1 \mathrm{H}), 7.40-7.33(\mathrm{~m}, 2 \mathrm{H}), 3.35(\mathrm{~s}, 3$ $\mathrm{H}), 0.94(\mathrm{~s}, 21 \mathrm{H})$

${ }^{13} \mathrm{C}$ NMR $\left(100 \mathrm{MHz}, \mathrm{CDCl}_{3}\right) \delta \mathrm{ppm} 171.4,133.8,131.7,128.7$ (2C), 127.9 (2C), 100.3, 70.0, 37.9, 18.6 (6C), $11.4(3 \mathrm{C})$

\section{N-benzyl-4-methyl-N-(2-triisopropylsilylethynyl)benzamide 2e}<smiles>CC(C)C(C)C(C)C</smiles>

Starting from N-benzyl-4-methyl-benzamide 1 e $(406 \mathrm{mg}, 1.8 \mathrm{mmol}), \mathrm{CuSO}_{4} .5 \mathrm{H}_{2} \mathrm{O}(71 \mathrm{mg}, 0.3 \mathrm{mmol}$, 0.15 equiv.), 1,10-phenanthroline (144 mg, $0.8 \mathrm{mmol}), \mathrm{K}_{3} \mathrm{PO}_{4}(1.02 \mathrm{~g}, 4.8 \mathrm{mmol})$ and 2bromoethynyl(triisopropyl)silane $(523 \mathrm{mg}, 2.0 \mathrm{mmol}$ ) under argon, using General procedure 1 and heating at $75^{\circ} \mathrm{C}$ for 4.5 days. The crude was purified to afford N-benzyl-4-methyl-N-(2triisopropylsilylethynyl)benzamide $\mathbf{2 e}$ as a colorless oil (335 $\mathrm{mg}, 41 \%$ yield).

IR (neat): 2166, 1676, 749, $675 \mathrm{~cm}^{-1}$

${ }^{1}$ H NMR $\left(400 \mathrm{MHz}, \mathrm{CDCl}_{3}\right) \delta$ ppm $7.76(\mathrm{~d}, J=8.1 \mathrm{~Hz}, 2 \mathrm{H}), 7.44(\mathrm{~d}, J=7.5 \mathrm{~Hz}, 2 \mathrm{H}), 7.35(\mathrm{~m}, 3 \mathrm{H}), 7.15$ $(\mathrm{d}, J=7.9 \mathrm{~Hz}, 2 \mathrm{H}), 4.85(\mathrm{~s}, 2 \mathrm{H}), 2.35(\mathrm{~s}, 3 \mathrm{H}), 0.88(\mathrm{~s}, 18 \mathrm{H}), 0.87(\mathrm{~s}, 3 \mathrm{H})$ 
${ }^{13} \mathrm{C}$ NMR $\left(100 \mathrm{MHz}, \mathrm{CDCl}_{3}\right) \delta \mathrm{ppm} 170.5,141.5,136.2,130.6,128.8,128.4,128.4,127.8,99.4,72.1$, 53.1, 41.6, 21.5, $18.4(6 \mathrm{C}), 11.4(3 \mathrm{C})$

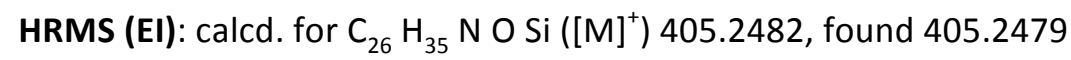

\section{N-benzyl-4-chloro-N-(2-triisopropylsilylethynyl)benzamide $2 f$}<smiles>CC(C)C(C)[Si](C#CCN(Cc1ccccc1)C(=O)c1ccc(Cl)cc1)(C(C)C)C(C)C</smiles>

Starting from N-benzyl-4-chloro-benzamide 1 f $\left(310 \mathrm{mg}, 1.3 \mathrm{mmol}\right.$ ), $\mathrm{CuSO}_{4} .5 \mathrm{H}_{2} \mathrm{O}(70 \mathrm{mg}, 0.3 \mathrm{mmol}$ ), 1,10-phenanthroline $(100 \mathrm{mg}, \quad 0.6 \mathrm{mmol}), \mathrm{K}_{3} \mathrm{PO}_{4}(0.71 \mathrm{~g}, \quad 3.0 \mathrm{mmol})$ and 2bromoethynyl(triisopropyl)silane $(370 \mathrm{mg}, 1.4 \mathrm{mmol}$ ) under argon, using General procedure 1 and heating at $75^{\circ} \mathrm{C}$ for 3 days. The crude was purified to afford N-benzyl-4-chloro-N-(2triisopropylsilylethynyl)benzamide $\mathbf{2 f}$ as a colorless oil ( $366 \mathrm{mg}, 67 \%$ yield).

IR (neat): 2168, 1681, 752, 699, $676 \mathrm{~cm}^{-1}$

${ }^{1}$ H NMR (400 MHz, DMSO- $\left.d_{6}\right) \delta$ ppm $7.73(d, J=8.3 \mathrm{~Hz}, 2 \mathrm{H}), 7.51(\mathrm{~d}, J=8.3 \mathrm{~Hz}, 2 \mathrm{H}), 7.35(\mathrm{~m}, 5 \mathrm{H})$, $4.82(\mathrm{~s}, 2 \mathrm{H}), 0.81(\mathrm{~s}, 18 \mathrm{H}), 0.81(\mathrm{~s}, 3 \mathrm{H})$

${ }^{13} \mathrm{C}$ NMR (100 MHz, DMSO- $\left.d_{6}\right) \delta \mathrm{ppm} 168.9,135.4,135,131.2,129.4$ (2C), 127.8 (2C), 127.7 (2C), $127.6(2 \mathrm{C}), 127.3,98.6,71,51.4,17.8(6 \mathrm{C}), 10.1(3 \mathrm{C})$

HRMS (EI): calcd. for $\mathrm{C}_{25} \mathrm{H}_{32} \mathrm{Cl} \mathrm{N} \mathrm{O} \mathrm{Si}\left([\mathrm{M}]^{+}\right)$425.1936, found 425.1939

\section{$\mathrm{N}$-benzyl-4-fluoro-N-(2-triisopropylsilylethynyl)benzamide 2g}<smiles>CC#C[Si](C(=O)c1ccc(F)cc1)(C(C)C)C(C)(C)C(C)C</smiles>

Starting from N-benzyl-4-fluoro-benzamide $1 \mathrm{~g}(2.1 \mathrm{~g}, 9.1 \mathrm{mmol}), \mathrm{CuSO}_{4} .5 \mathrm{H}_{2} \mathrm{O}(161 \mathrm{mg}, 0.9 \mathrm{mmol})$, 1,10-phenanthroline $327 \mathrm{mg}, \quad 1.8 \mathrm{mmol}), \mathrm{K}_{3} \mathrm{PO}_{4}(2.43 \mathrm{~g}, 18.2 \mathrm{mmol})$ and 2bromoethynyl(triisopropyl)silane $(2.61 \mathrm{~g}, 10.0 \mathrm{mmol}, 1.1$ equiv.) under argon, using General procedure $1^{\prime}$ and heating at $75^{\circ} \mathrm{C}$ for 3.5 days. The crude was purified to afford N-benzyl-4-fluoro- $\mathrm{N}$ (2-triisopropylsilylethynyl)benzamide $\mathbf{2 g}$ as a colorless oil ( $1.72 \mathrm{~g}$, $46 \%$ yield).

IR (neat): 2166, 1677, $1604 \mathrm{~cm}^{-1}$

${ }^{1}$ H NMR $\left(400 \mathrm{MHz}\right.$, DMSO- $\left.d_{6}\right) \delta$ ppm $7.8(\mathrm{~m}, 2 \mathrm{H}), 7.4(\mathrm{~m}, 5 \mathrm{H}), 7.3(\mathrm{~m}, 2 \mathrm{H}), 4.8(\mathrm{~s}, 2 \mathrm{H}), 0.8(\mathrm{~m}, 21 \mathrm{H})$

${ }^{13} \mathrm{C}$ NMR (100 MHz, DMSO- $\left.d_{6}\right) \delta \mathrm{ppm} 169.7,164.1$ (d, $\left.J=250 \mathrm{~Hz}\right), 136.1,131.4(\mathrm{~d}, J=9.0 \mathrm{~Hz}, 2 \mathrm{C})$, $130.4(d, J=3.1 \mathrm{~Hz}), 128.9(2 \mathrm{C}), 128.9(2 \mathrm{C}), 128.5,115.6(\mathrm{~d}, J=22.3 \mathrm{~Hz}, 2 \mathrm{C}), 99.3,71.7,52.3,18.7$ (6C), $11(3 C)$ 
${ }^{19}$ F NMR $\left(376 \mathrm{MHz}\right.$, DMSO- $\left.d_{6}\right) \delta \mathrm{ppm}-108.5$

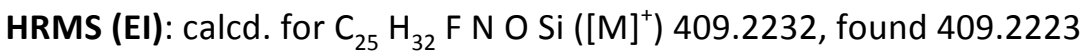

\section{N-benzyl-4-(trifluoromethyl)-N-(2-triisopropylsilylethynyl)benzamide $2 \mathrm{~h}$}<smiles>CC(C)[Si](C#CN(Cc1ccccc1)C(=O)c1ccc(C(F)(F)F)cc1)(C(C)C)C(C)C</smiles>

Starting from N-benzyl-4-(trifluoromethyl)benzamide $1 \mathrm{~h}(350 \mathrm{mg}, 1.3 \mathrm{mmol}), \mathrm{CuSO}_{4} .5 \mathrm{H}_{2} \mathrm{O}(70 \mathrm{mg}, 0.3$ $\mathrm{mmol})$, 1,10-phenanthroline $(100 \mathrm{mg}, 0.6 \mathrm{mmol}), \mathrm{K}_{3} \mathrm{PO}_{4}(0.71 \mathrm{~g}, 3.0 \mathrm{mmol})$ and 2bromoethynyl(triisopropyl)silane $(370 \mathrm{mg}, 1.4 \mathrm{mmol}$ ) under argon using General procedure 1 and heating at $75^{\circ} \mathrm{C}$ for 3 days. The crude was purified to afford N-benzyl-4-(trifluoromethyl)- $\mathrm{N}-(2-$ triisopropylsilylethynyl)benzamide $\mathbf{2 h}$ as a colorless oil ( $355 \mathrm{mg}, 61 \%$ yield).

IR (neat): $2169,1684,1170,1131,744,698,676 \mathrm{~cm}^{-1}$

${ }^{1}$ H NMR (400 MHz, DMSO- $\left.d_{6}\right) \delta$ ppm $7.87(\mathrm{~d}, J=8.3 \mathrm{~Hz}, 2 \mathrm{H}), 7.81(\mathrm{~d}, J=8.3 \mathrm{~Hz}, 2 \mathrm{H}), 7.44-7.31(\mathrm{~m}, 5$ $\mathrm{H}), 4.85(\mathrm{~s}, 2 \mathrm{H}), 0.75(\mathrm{~s}, 18 \mathrm{H}), 0.75(\mathrm{~s}, 3 \mathrm{H})$

${ }^{13} \mathrm{C}$ NMR $\left(100 \mathrm{MHz}, \mathrm{DMSO}-d_{6}\right) \delta \mathrm{ppm} 169.8,138.3,135.9,131.4$ (q, J = $\left.32 \mathrm{~Hz}\right), 129.0(4 \mathrm{C}), 129.0(2 \mathrm{C})$, $128.4,125.7$ (q, $J=3.8 \mathrm{~Hz}, 2 \mathrm{C}), 124.4(q, J=273 \mathrm{~Hz}), 98.7,72.4,51.8,18.2(6 \mathrm{C}), 10.9$ (3C)

${ }^{19}$ F NMR $\left(376 \mathrm{MHz}, \mathrm{DMSO}-d_{6}\right) \delta \mathrm{ppm}-61.7$

HRMS (ESI): calcd. for $\mathrm{C}_{26} \mathrm{H}_{33} \mathrm{~F}_{3} \mathrm{~N} O \mathrm{Si}\left([\mathrm{M}+\mathrm{H}]^{+}\right) 460.2278$, found 460.228

\section{N-benzyl-4-tert-butyl-N-(2-triisopropylsilylethynyl)benzamide 2i}<smiles>C#C[Si](C(=O)c1ccc(C(C)(C)C)cc1)(C(C)C)C(C)C</smiles>

Starting from N-benzyl-4-tert-butyl-benzamide $1 \mathrm{i}(340 \mathrm{mg}, 1.3 \mathrm{mmol}), \mathrm{CuSO}_{4} .5 \mathrm{H}_{2} \mathrm{O}(70 \mathrm{mg}, 0.3$ $\mathrm{mmol})$, 1,10-phenanthroline (100 mg, $0.6 \mathrm{mmol}), \mathrm{K}_{3} \mathrm{PO}_{4}(0.71 \mathrm{~g}, 3.0 \mathrm{mmol})$ and 2bromoethynyl(triisopropyl)silane $(370 \mathrm{mg}, 1.4 \mathrm{mmol}$ ) under argon, using General procedure 1 and heating at $75^{\circ} \mathrm{C}$ for 3 days. The crude was purified to afford N-benzyl-4-tert-butyl- $\mathrm{N}-(2-$ triisopropylsilylethynyl)benzamide $\mathbf{2} \mathbf{i}$ as a colorless oil ( $215 \mathrm{mg}, 38 \%$ yield).

IR (neat): $2167,1678,770,747,697 \mathrm{~cm}^{-1}$

${ }^{1}$ H NMR (500 MHz, DMSO-d $\left.d_{6}\right) \delta$ ppm $7.64(\mathrm{~d}, J=8.3 \mathrm{~Hz}, 2 \mathrm{H}), 7.44$ (d, $\left.J=8.3 \mathrm{~Hz}, 2 \mathrm{H}\right), 7.41-7.30(\mathrm{~m}, 5$ $\mathrm{H}), 4.81(\mathrm{~s}, 2 \mathrm{H}), 1.28(\mathrm{~s}, 9 \mathrm{H}), 0.8(\mathrm{~s}, 18 \mathrm{H}), 0.8(\mathrm{~s}, 3 \mathrm{H})$ 
${ }^{13} \mathrm{C}$ NMR $\left(125 \mathrm{MHz}, \mathrm{DMSO}-d_{6}\right) \delta \mathrm{ppm} 170.7,154.3,136.1,130.5,128.8(2 \mathrm{C}), 128.7(2 \mathrm{C}), 128.3(2 \mathrm{C})$, $128.2,125.2(2 \mathrm{C}), 99,71.4,52.2,34.6,31.1(3 \mathrm{C}), 18.8(6 \mathrm{C}), 10.9$ (3C)

HRMS (EI): calcd. for $\mathrm{C}_{29} \mathrm{H}_{41} \mathrm{~N} O \mathrm{Si}\left([\mathrm{M}]^{+}\right)$447.2952, found 447.2942

\section{N-benzyl-4-nitro-N-(2-triisopropylsilylethynyl)benzamide 2j<smiles>CC(C)[Si](C#CN(Cc1ccccc1)C(=O)c1ccc([N+](=O)[O-])cc1)(C(C)C)C(C)C</smiles>

Starting from N-benzyl-4-nitro-benzamide $1 \mathrm{j}(320 \mathrm{mg}, 1.3 \mathrm{mmol}), \mathrm{CuSO}_{4} .5 \mathrm{H}_{2} \mathrm{O}(70 \mathrm{mg}, 0.3 \mathrm{mmol})$, 1,10-phenanthroline $(100 \mathrm{mg}, 0.6 \mathrm{mmol}), \quad \mathrm{K}_{3} \mathrm{PO}_{4}(0.71 \mathrm{~g}, \quad 3.0 \mathrm{mmol})$ and 2bromoethynyl(triisopropyl)silane $(370 \mathrm{mg}, 1.4 \mathrm{mmol}$ ) under argon, using General procedure 1 and heating at $75^{\circ} \mathrm{C}$ for 3 days. The crude was purified to afford N-benzyl-4-nitro-N-(2triisopropylsilylethynyl)benzamide $\mathbf{2} \mathbf{j}$ as a colorless oil ( $408 \mathrm{mg}, 73 \%$ yield).

IR (neat): 2169, 1680, 1525, 1346, 851, 719, $698 \mathrm{~cm}^{-1}$

${ }^{1} \mathrm{H}$ NMR $\left(500 \mathrm{MHz}, \mathrm{DMSO}-d_{6}\right) \delta \mathrm{ppm} 8.3(\mathrm{~d}, J=8.5 \mathrm{~Hz}, 2 \mathrm{H}), 7.95(\mathrm{~d}, J=8.5 \mathrm{~Hz}, 2 \mathrm{H}), 7.43-7.37(\mathrm{~m}, 4$ H), $7.36(\mathrm{t}, J=6.9 \mathrm{~Hz}, 1 \mathrm{H}), 4.85(\mathrm{~s}, 2 \mathrm{H}), 0.75(\mathrm{~s}, 18 \mathrm{H}), 0.75(\mathrm{~s}, 3 \mathrm{H})$

${ }^{13} \mathrm{C}$ NMR $\left(125 \mathrm{MHz}, \mathrm{DMSO}-d_{6}\right) \delta$ ppm $168.3,148,139.4,134.7,128.7$ (2C), 127.9 (2C), 127.9 (2C), $127.4,122.8(2 \mathrm{C}), 97.4,71.7,50.9,17.5$ (6C), 9.9 (3C)

HRMS (EI): calcd. for $\mathrm{C}_{25} \mathrm{H}_{32} \mathrm{~N}_{2} \mathrm{O}_{3} \mathrm{Si}\left([\mathrm{M}]^{+}\right) 436.2177$, found 436.2179

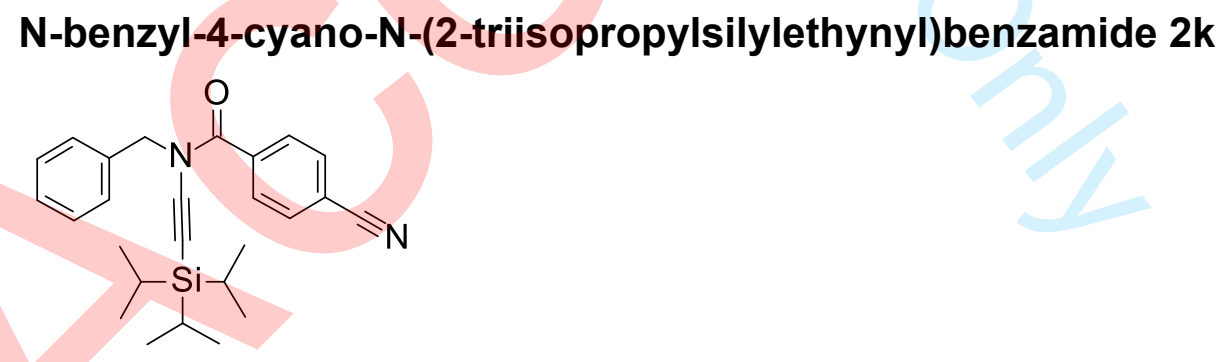

Starting from N-benzyl-4-cyano-N-ethynyl-benzamide $1 \mathbf{k}(360 \mathrm{mg}, 1.5 \mathrm{mmol}), \mathrm{CuSO}_{4} .5 \mathrm{H}_{2} \mathrm{O}(53 \mathrm{mg}$, $0.3 \mathrm{mmol}$ ), 1,10-phenanthroline (110 mg, $0.6 \mathrm{mmol}), \mathrm{K}_{3} \mathrm{PO}_{4}(0.48 \mathrm{~g}, 3.6 \mathrm{mmol}, 2.4$ equiv.) and 2bromoethynyl(triisopropyl)silane (390 $\mathrm{mg}, 30.0 \mathrm{mmol}, 0.8$ equiv.) under argon, using General procedure 1 and heating at $75^{\circ} \mathrm{C}$ for 3 days. The crude was purified to afford N-benzyl-4-cyano- $\mathrm{N}-(2-$ triisopropylsilylethynyl)benzamide $\mathbf{2 k}$ as a colorless oil ( $232 \mathrm{mg}, 37 \%$ yield).

IR (neat): 2231, 2169, 1679, 1609, $1273 \mathrm{~cm}^{-1}$

${ }^{1} \mathrm{H}$ NMR $\left(400 \mathrm{MHz}, \mathrm{DMSO}-d_{6}\right) \delta \mathrm{ppm} 7.97$ (d, $\left.J=8.4 \mathrm{~Hz}, 2 \mathrm{H}\right), 7.88(\mathrm{~d}, J=8.4 \mathrm{~Hz}, 2 \mathrm{H}), 7.35(\mathrm{~m}, 5 \mathrm{H})$, $4.82(\mathrm{~s}, 2 \mathrm{H}), 0.8(\mathrm{~m}, 21 \mathrm{H})$

${ }^{13} \mathrm{C}$ NMR (100 MHz, DMSO- $d_{6}$ ) $\delta$ ppm 169.6, 138.7, 135.8, 132.8, 129.1 (2C), 129.0 (2C), $128.9(2 \mathrm{C})$, $128.5,128.5,118.5,113.8,98.5,72.6,52,18.7$ (6C), 11 (3C) 
HRMS (EI): calcd. for $\mathrm{C}_{26} \mathrm{H}_{32} \mathrm{~N}_{2} \mathrm{O}$ Si $\left([\mathrm{M}]^{+}\right)$416.2278, found 416.2279

\section{methyl 4-[benzyl(2-triisopropylsilylethynyl)carbamoyl]benzoate 2I}<smiles>COC(=O)c1ccc(C(=O)N(C#CC(C)C(C)C)Cc2ccccc2)cc1</smiles>

Starting from methyl 4-(benzylcarbamoyl)benzoate $1 \mathrm{l}\left(340 \mathrm{mg}, 1.3 \mathrm{mmol}\right.$ ), $\mathrm{CuSO}_{4} .5 \mathrm{H}_{2} \mathrm{O}(70 \mathrm{mg}, 0.3$ $\mathrm{mmol})$, 1,10-phenanthroline $(100 \mathrm{mg}, 0.6 \mathrm{mmol}), \mathrm{K}_{3} \mathrm{PO}_{4}(0.71 \mathrm{~g}, 3.0 \mathrm{mmol})$ and 2bromoethynyl(triisopropyl)silane $(370 \mathrm{mg}, 1.4 \mathrm{mmol}$ ) under argon, using General procedure 1 and heating at $75^{\circ} \mathrm{C}$ for 3 days. The crude was purified to afford methyl 4-[benzyl(2triisopropylsilylethynyl)carbamoyl]benzoate $\mathbf{2 l}$ as a colorless oil (315 $\mathrm{mg}, 55 \%$ yield).

IR (neat): $2168,1728,1678,1271,1106,727,699,675 \mathrm{~cm}^{-1}$

${ }^{1}$ H NMR $\left(500 \mathrm{MHz}\right.$, DMSO- $\left.d_{6}\right) \delta \mathrm{ppm} 8.00 / 7.82(2 \mathrm{~d}, J=8.2 \mathrm{~Hz}, 4 \mathrm{H}), 7.43-7.31(\mathrm{~m}, 5 \mathrm{H}), 4.84(\mathrm{~s}, 2 \mathrm{H})$, $3.88(\mathrm{~s}, 3 \mathrm{H}), 0.78(\mathrm{~s}, 18 \mathrm{H}), 0.78(\mathrm{~s}, 3 \mathrm{H})$

${ }^{13}$ C NMR (125 MHz, DMSO-d $\left.d_{6}\right) \delta$ ppm 169, 164.9, 137.5, 135, 131.1, 128.3 (2C), 127.9 (2C), $127.8(2 \mathrm{C})$, $127.6(2 \mathrm{C}), 127.4,97.8,71.4,51.9,51,17.5(6 \mathrm{C}), 10.9$ (3C)

HRMS (EI): calcd. for $\mathrm{C}_{27} \mathrm{H}_{35} \mathrm{~N} \mathrm{O}_{3} \mathrm{Si}\left([\mathrm{M}]^{+}\right)$449.2381, found 449.2378

\section{N-benzyl-N-(2-triisopropylsilylethynyl)pyridine-4-carboxamide $2 \mathrm{~m}$}<smiles>CC(C)[Si](C(C)C)(C(C)C)C(C)C</smiles>

Starting from N-benzylpyridine-4-carboxamide $1 \mathrm{~m}(382 \mathrm{mg}, 1.8 \mathrm{mmol}), \mathrm{CuSO}_{4} .5 \mathrm{H}_{2} \mathrm{O}(71 \mathrm{mg}, 0.3$ mmol, 0.15 equiv.), 1,10-phenanthroline (144 mg, $0.8 \mathrm{mmol}), \mathrm{K}_{3} \mathrm{PO}_{4}(1.02 \mathrm{~g}, 4.8 \mathrm{mmol})$ and 2bromoethynyl(triisopropyl)silane $(523 \mathrm{mg}, 2.0 \mathrm{mmol}$ ) under argon, using General procedure 1 and heating at $75^{\circ} \mathrm{C}$ for 4.5 days. The crude was purified to afford N-benzyl-N-(2triisopropylsilylethynyl)pyridine-4-carboxamide $\mathbf{2 m}$ as a yellow oil ( $514 \mathrm{mg}$, $73 \%$ yield).

IR (neat): $2170,1683,753,673 \mathrm{~cm}^{-1}$

${ }^{1} \mathrm{H}$ NMR $\left(400 \mathrm{MHz}, \mathrm{CDCl}_{3}\right) \delta \mathrm{ppm} 8.68(\mathrm{~d}, J=4.9 \mathrm{~Hz}, 2 \mathrm{H}), 7.6(\mathrm{~d}, J=4.8 \mathrm{~Hz}, 2 \mathrm{H}), 7.43(\mathrm{dd}, J=7.6,1.8$ $\mathrm{Hz}, 2 \mathrm{H}), 7.35(\mathrm{~m}, 3 \mathrm{H}), 4.86(\mathrm{~s}, 2 \mathrm{H}), 0.89(\mathrm{~s}, 18 \mathrm{H}), 0.89(\mathrm{~s}, 3 \mathrm{H})$

${ }^{13} \mathrm{C} \mathrm{NMR}\left(100 \mathrm{MHz}, \mathrm{CDCl}_{3}\right) \delta \mathrm{ppm} 168.6,149.8$ (2C), 141.4, 135.2, 129.0 (2C), 128.7 (2C), 128.3, 122.9 (2C), 97.7, 73.9, 52.9, 18.9 (6C), 11.6 (3C)

HRMS (EI): calcd. for $\mathrm{C}_{24} \mathrm{H}_{32} \mathrm{~N}_{2} \mathrm{O} \mathrm{Si}\left([\mathrm{M}]^{+}\right)$392.2278, found 392.228 


\section{N-benzyl-N-(2-triisopropylsilylethynyl)-1,3-benzothiazole-6-carboxamide 2n}<smiles>CC(C)[Si](C#CN(Cc1ccccc1)C(=O)c1ccc2ncsc2c1)(C(C)C)C(C)C</smiles>

Starting from N-benzyl-1,3-benzothiazole-6-carboxamide $1 \mathrm{n}\left(340 \mathrm{mg}, 1.3 \mathrm{mmol}\right.$ ), $\mathrm{CuSO}_{4} .5 \mathrm{H}_{2} \mathrm{O}$ (70 $\mathrm{mg}, 0.3 \mathrm{mmol}), 1,10$-phenanthroline $(100 \mathrm{mg}, 0.6 \mathrm{mmol}), \mathrm{K}_{3} \mathrm{PO}_{4}(0.71 \mathrm{~g}, 3.0 \mathrm{mmol})$ and 2bromoethynyl(triisopropyl)silane $(370 \mathrm{mg}, 1.4 \mathrm{mmol}$ ) under argon, using General procedure 1 and heating at $75^{\circ} \mathrm{C}$ for 3 days. The crude was purified to afford N-benzyl-N-(2-triisopropylsilylethynyl)1,3-benzothiazole-6-carboxamide $\mathbf{2 n}$ as a colorless oil ( $401 \mathrm{mg}, 70 \%$ yield).

IR (neat): 2167, 1674, 877, 740, 698, $675 \mathrm{~cm}^{-1}$

${ }^{1} \mathrm{H}$ NMR $\left(500 \mathrm{MHz}\right.$, DMSO-d $\left.d_{6}\right) \delta \mathrm{ppm} 9.53(\mathrm{~s}, 1 \mathrm{H}), 8.6(\mathrm{~d}, J=1.4 \mathrm{~Hz}, 1 \mathrm{H}), 8.13(\mathrm{~d}, J=8.5 \mathrm{~Hz}, 1 \mathrm{H}), 7.83$ (dd, $J=8.5,1.6 \mathrm{~Hz}, 1 \mathrm{H}), 7.44(\mathrm{~d}, J=7.4 \mathrm{~Hz}, 2 \mathrm{H}), 7.39(\mathrm{t}, J=7.3 \mathrm{~Hz}, 2 \mathrm{H}), 7.34(\mathrm{t}, J=7.3 \mathrm{~Hz}, 1 \mathrm{H}), 4.88$ $(\mathrm{s}, 2 \mathrm{H}), 0.74(\mathrm{~s}, 18 \mathrm{H}), 0.74(\mathrm{~s}, 3 \mathrm{H})$

${ }^{13}$ C NMR $\left(125 \mathrm{MHz}\right.$, DMSO- $\left.d_{6}\right) \delta$ ppm 170.3, 159.5, 154.9, 136.1, 133.6, 130.9, $129.0(2 \mathrm{C}), 128.9$ (2C), $128.4,126.5,123.5,123.1,99.3,71.8,52.3,18.6(6 \mathrm{C}), 11(3 \mathrm{C})$

HRMS (EI): calcd. for $\mathrm{C}_{26} \mathrm{H}_{32} \mathrm{~N}_{2} \mathrm{O} S \mathrm{Si}\left([\mathrm{M}]^{+}\right)$448.1999, found 448.1977

\section{N-benzyl-3,5-difluoro-N-(2-triisopropylsilylethynyl)benzamide 20}<smiles>CC(C)[Si](C#CN(Cc1ccccc1)C(=O)c1cc(F)cc(F)c1)(C(C)C)C(C)C</smiles>

Starting from N-benzyl-3,5-difluoro-benzamide 10 (310 mg, $1.3 \mathrm{mmol}$ ), $\mathrm{CuSO}_{4} .5 \mathrm{H}_{2} \mathrm{O}(70 \mathrm{mg}, 0.3$ $\mathrm{mmol})$, 1,10-phenanthroline (100 $\mathrm{mg}, 0.6 \mathrm{mmol}), \mathrm{K}_{3} \mathrm{PO}_{4}(0.71 \mathrm{~g}, 3.0 \mathrm{mmol})$ and 2bromoethynyl(triisopropyl)silane $(370 \mathrm{mg}, 1.4 \mathrm{mmol}$ ) under argon, using General procedure 1 and heating at $75^{\circ} \mathrm{C}$ for 3 days. The crude was purified to afford N-benzyl-3,5-difluoro- $\mathrm{N}-(2-$ triisopropylsilylethynyl)benzamide $\mathbf{2 0}$ as a colorless oil ( $333 \mathrm{mg}, 61 \%$ yield).

IR (neat): 2169, 1680, 1594, 751, 698, $675 \mathrm{~cm}^{-1}$

${ }^{1}$ H NMR $\left(400 \mathrm{MHz}\right.$, DMSO-d $\left.d_{6}\right) \delta$ ppm $7.46(\mathrm{~m}, 2 \mathrm{H}), 7.39(\mathrm{~m}, 1 \mathrm{H}), 7.36(\mathrm{~m}, 5 \mathrm{H}), 4.82(\mathrm{~s}, 2 \mathrm{H}), 0.81(\mathrm{~s}$, $18 \mathrm{H}), 0.81(\mathrm{~s}, 3 \mathrm{H})$

${ }^{13} \mathrm{C}$ NMR $\left(100 \mathrm{MHz}, \mathrm{DMSO}-d_{6}\right) \delta \mathrm{ppm} 168.5,162.4$ (dd, $\left.J=247.9,12.4 \mathrm{~Hz}, 2 \mathrm{C}\right), 137.5(\mathrm{t}, J=9.2 \mathrm{~Hz})$, $135.8,129.2(4 \mathrm{C}), 128.5,112.1(\mathrm{~d}, J=27.2 \mathrm{~Hz}, 2 \mathrm{C}), 107.0(\mathrm{t}, J=25.9 \mathrm{~Hz}), 98.6,72.7,52.2,18.8(6 \mathrm{C})$, $11.1(3 \mathrm{C})$ 
${ }^{19}$ F NMR (376 MHz, DMSO- $\left.d_{6}\right) \delta-108.9 \mathrm{ppm}$

HRMS (EI): calcd. for $\mathrm{C}_{25} \mathrm{H}_{31} \mathrm{~F}_{2} \mathrm{~N} O \mathrm{Si}\left([\mathrm{M}]^{+}\right)$427.2137, found 427.2135

\section{$\mathrm{N}$-benzyl-2-methoxy-N-(2-triisopropylsilylethynyl)benzamide 2p}<smiles>COc1ccccc1C(=O)N(C#C[Si](C(C)C)(C(C)C)C(C)C)Cc1ccccc1</smiles>

Starting from N-benzyl-2-methoxy-benzamide $1 p$ (434 mg, $1.8 \mathrm{mmol}$ ), $\mathrm{CuSO}_{4} .5 \mathrm{H}_{2} \mathrm{O}(71 \mathrm{mg}, 0.3 \mathrm{mmol}$, 0.15 equiv.), 1,10-phenanthroline (144 mg, $0.8 \mathrm{mmol}), \mathrm{K}_{3} \mathrm{PO}_{4}(1.02 \mathrm{~g}, 4.8 \mathrm{mmol})$ and 2bromoethynyl(triisopropyl)silane $(523 \mathrm{mg}, 2.0 \mathrm{mmol}$ ) under argon, using General procedure 1 and heating at $75^{\circ} \mathrm{C}$ for 9.5 days. The crude was purified to afford $\mathrm{N}$-benzyl-2-methoxy- $\mathrm{N}-(2-$ triisopropylsilylethynyl)benzamide $2 p$ as a colorless oil ( $447 \mathrm{mg}, 59 \%$ yield).

IR (neat): 2172, 1686, 1251, 750, $675 \mathrm{~cm}^{-1}$

${ }^{1} \mathbf{H}$ NMR $\left(400 \mathrm{MHz}\right.$, DMSO-d $\left.d_{6}\right) \delta \mathrm{ppm} 7.38(\mathrm{~m}, 5 \mathrm{H}), 7.33(\mathrm{~m}, 1 \mathrm{H}), 7.29(\mathrm{dd}, J=8.4,1.5 \mathrm{~Hz}, 1 \mathrm{H}), 7.08$ $(\mathrm{d}, J=8.0 \mathrm{~Hz}, 1 \mathrm{H}), 6.97(\mathrm{t}, J=7.7 \mathrm{~Hz}, 1 \mathrm{H}), 4.82(\mathrm{~s}, 2 \mathrm{H}), 3.79(\mathrm{~s}, 3 \mathrm{H}), 0.73(\mathrm{~s}, 18 \mathrm{H}), 0.73(\mathrm{~s}, 3 \mathrm{H})$

${ }^{13} \mathrm{C}$ NMR $\left(100 \mathrm{MHz}, \mathrm{DMSO}-d_{6}\right) \delta$ ppm 170.1, 156.1, 136.2, 131.8, 128.8 (2C), 128.4 (2C), 128.1, 127.9, $125.2,120.7,111.9,99.1,70,56.1,51.3,18.6(6 C), 11(3 C)$

HRMS (EI): calcd. for $\mathrm{C}_{26} \mathrm{H}_{35} \mathrm{~N} \mathrm{O}_{2} \mathrm{Si}\left([\mathrm{M}]^{+}\right)$421.2432, found 421.2416

\section{N-(3-methoxypropyl)-N-(2-triisopropylsilylethynyl)benzamide 2q}

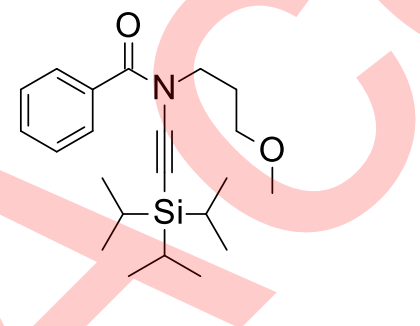

Starting from $\mathrm{N}$-(3-methoxypropyl)benzamide 1q $(500 \mathrm{mg}, 2.6 \mathrm{mmol}), \mathrm{CuSO}_{4} .5 \mathrm{H}_{2} \mathrm{O}(129 \mathrm{mg}, 0.5$ $\mathrm{mmol}), 1,10$-phenanthroline $(187 \mathrm{mg}, 1.0 \mathrm{mmol}), \mathrm{K}_{3} \mathrm{PO}_{4}(0.69 \mathrm{~g}, 5.2 \mathrm{mmol}, 2.0$ equiv.) and 2bromoethynyl(triisopropyl)silane $(0.74 \mathrm{~g}, 2.8 \mathrm{mmol})$ under argon, using General procedure 1 and heating at $75^{\circ} \mathrm{C}$ for 5 days. The crude was purified to afford $\mathrm{N}$-(3-methoxypropyl)-N-(2triisopropylsilylethynyl)benzamide $\mathbf{2 q}$ as a yellow oil ( $189 \mathrm{mg}, 20 \%$ yield).

IR (neat): $2165,1678,1278,1118,709,673 \mathrm{~cm}^{-1}$

${ }^{1} \mathrm{H}$ NMR $\left(500 \mathrm{MHz}\right.$, DMSO- $\left.d_{6}\right) \delta \mathrm{ppm} 7.67(\mathrm{~d}, J=7.6 \mathrm{~Hz}, 2 \mathrm{H}), 7.52(\mathrm{t}, J=7.5 \mathrm{~Hz}, 1 \mathrm{H}), 7.43(\mathrm{t}, J=7.4$ $\mathrm{Hz}, 2 \mathrm{H}), 3.7(\mathrm{t}, J=6.8 \mathrm{~Hz}, 2 \mathrm{H}), 3.44(\mathrm{t}, J=6.3 \mathrm{~Hz}, 2 \mathrm{H}), 3.24(\mathrm{~s}, 3 \mathrm{H}), 1.95$ (quint, $J=6.6 \mathrm{~Hz}, 2 \mathrm{H}), 0.9(\mathrm{~s}$, $18 \mathrm{H}), 0.9(\mathrm{~s}, 3 \mathrm{H})$ 
${ }^{13} \mathrm{C}$ NMR $\left(100 \mathrm{MHz}, \mathrm{DMSO}-d_{6}\right) \delta \mathrm{ppm} 170.9,134.2,131.6,128.5(2 \mathrm{C}), 128.4(2 \mathrm{C}), 99.6,71.3,69.5$, $58.6,46.4,27.7,18.8(6 \mathrm{C}), 11.2(3 \mathrm{C})$

HRMS (ESI): calcd. for $\mathrm{C}_{22} \mathrm{H}_{36} \mathrm{~N} \mathrm{O}_{2} \mathrm{Si}\left([\mathrm{M}+\mathrm{H}]^{+}\right)$374.2437, found 374.2512

\section{General procedure 2 for the synthesis of ynamides 3a-q}

To a solution of the protected ynamide 2 (1 equiv.) in tetrahydrofuran $(0.2 \mathrm{M})$ under argon at $0^{\circ} \mathrm{C} /-$ $5^{\circ} \mathrm{C}$ was added tetrabutylammonium fluoride ( $1 \mathrm{M}$ in THF, 1.2 equiv.) dropwise.

The reaction mixture was stirred at $0^{\circ} \mathrm{C}$ for the given amount of time.

After completion of the reaction, a mixture of an aqueous solution of $\mathrm{NH}_{4} \mathrm{Cl}(10 \% \mathrm{w} / \mathrm{w})$ was added, the aqueous phase was extracted three times with diethyl ether. After washing with a saturated solution of $\mathrm{NaCl}$, the organic phases were dried over $\mathrm{Na}_{2} \mathrm{SO}_{4}$ and concentrated.

The crude was purified either by trituration in pentane at $-20^{\circ} \mathrm{C}$ (if $400 \mathrm{mg}$ of crude obtained, $4 \mathrm{~mL}$ of pentane was added) or by silica gel flash column chromatography (gradient eluent, EtOAc in heptane).

\section{N-benzyl-N-ethynyl-benzamide $3 a$}<smiles>C#CN(Cc1ccccc1)C(=O)c1ccccc1</smiles>

Starting from N-benzyl-N-(2-triisopropylsilylethynyl)benzamide 2 a (1.33 g, $3.4 \mathrm{mmol}$ ), TBAF 1M/THF $\left(4.1 \mathrm{~mL}, 4.1 \mathrm{mmol}\right.$ ) under argon, using General procedure 2 and stirring at $0^{\circ} \mathrm{C}$ for $1 \mathrm{~h}$. The crude was purified by silica gel flash column chromatography to afford N-benzyl-N-ethynyl-benzamide 3a as a white solid (699 mg, $87 \%$ yield). Spectral data are in accordance with those reported in the literature. $^{5}$

IR (neat): $3242,2146,1649 \mathrm{~cm}^{-1}$

${ }^{1} \mathbf{H}$ NMR $(400 \mathrm{MHz}, \text { DMSO-d })_{6} \delta \mathrm{ppm} 7.73(\mathrm{~d}, J=7.7 \mathrm{~Hz}, 2 \mathrm{H}), 7.55(\mathrm{t}, J=7.5 \mathrm{~Hz}, 1 \mathrm{H}), 7.48(\mathrm{t}, J=7.5 \mathrm{~Hz}$, $2 \mathrm{H}), 7.4(\mathrm{~m}, 4 \mathrm{H}), 7.32(\mathrm{~m}, 1 \mathrm{H}), 4.82(\mathrm{~s}, 2 \mathrm{H}), 3.82(\mathrm{~s}, 1 \mathrm{H})$

${ }^{13} \mathrm{C}$ NMR $\left(125 \mathrm{MHz}, \mathrm{DMSO}-d_{6}\right) \delta \mathrm{ppm} 170.6,136.5,133.7,132,129.0(2 \mathrm{C}), 128.6(2 \mathrm{C}), 128.5$ (2C), $128.4(2 \mathrm{C}), 128.3,78.9,64.2,52.3$

HRMS (ESI): calcd. for $\mathrm{C}_{16} \mathrm{H}_{14} \mathrm{NO}\left([\mathrm{M}+\mathrm{H}]^{+}\right)$236.1070, found 236.1071

$m p: 45.5-48^{\circ} \mathrm{C}$

\footnotetext{
${ }^{5}$ B. Witulski, T. Stengel, Angew. Chem. Int. Ed. 1998, 37, 489
} 


\section{N-ethynyl-N-[(4-methoxyphenyl)methyl]benzamide 3b}<smiles>C=CN(Cc1ccc(OC)cc1)C(=O)c1ccccc1</smiles>

Starting from $\mathrm{N}$-[(4-methoxyphenyl)methyl]-N-(2-triisopropylsilylethynyl)benzamide 2 b (1.87 g, 4.4 $\mathrm{mmol})$, TBAF $1 \mathrm{M} / \mathrm{THF}(5.3 \mathrm{~mL}, 5.3 \mathrm{mmol})$ under argon, using General procedure 2 and stirring at $0^{\circ} \mathrm{C}$ for $10 \mathrm{~min}$. The crude was purified by silica gel flash column chromatography to afford $\mathrm{N}$-ethynyl-N[(4-methoxyphenyl)methyl]benzamide $\mathbf{3 b}$ as a white solid ( $806 \mathrm{mg}, 69 \%$ yield).

IR (neat): 3242, 2142, $1645 \mathrm{~cm}^{-1}$

${ }^{1} \mathrm{H}$ NMR $\left(500 \mathrm{MHz}, \mathrm{CDCl}_{3}\right) \delta \mathrm{ppm} 7.8(\mathrm{~d}, J=7.6 \mathrm{~Hz}, 2 \mathrm{H}), 7.48(\mathrm{t}, J=7.4 \mathrm{~Hz}, 1 \mathrm{H}), 7.39(\mathrm{~m}, 4 \mathrm{H}), 6.9(\mathrm{~d}, J$ $=8.2 \mathrm{~Hz}, 2 \mathrm{H}), 4.8(\mathrm{~s}, 2 \mathrm{H}), 3.81(\mathrm{~s}, 3 \mathrm{H}), 2.75(\mathrm{~s}, 1 \mathrm{H})$

${ }^{13} \mathrm{C}$ NMR $\left(125 \mathrm{MHz}, \mathrm{CDCl}_{3}\right) \delta \mathrm{ppm} 170.75,159.51,133.41,131.45,130.26$ (2C), 128.68 (2C), 128.06, $127.8(2 \mathrm{C}), 113.9(2 \mathrm{C}), 78.62,61.7,55.27,52.25$

HRMS (ESI): calcd. for $\mathrm{C}_{17} \mathrm{H}_{16} \mathrm{~N} \mathrm{O}_{2}\left([\mathrm{M}+\mathrm{H}]^{+}\right)$266.1176, found 266.1177

$\mathrm{mp}: 86.8-88.7^{\circ} \mathrm{C}$

\section{$\mathrm{N}$-[(2,4-dimethoxyphenyl)methyl]-N-ethynyl-benzamide 3c}<smiles>C#CN(Cc1ccc(OC)cc1OC)C(=O)c1ccccc1</smiles>

Starting from N-[(2,4-dimethoxyphenyl)methyl]-N-(2-triisopropylsilylethynyl)benzamide 2c (405 mg, $0.90 \mathrm{mmol})$, TBAF $1 \mathrm{M} / \mathrm{THF}(1.08 \mathrm{~mL}, 1.08 \mathrm{mmol})$ under argon, using General procedure 2 and stirring at $0^{\circ} \mathrm{C}$ for $30 \mathrm{~min}$. The crude was purified by trituration in pentane to afford $\mathrm{N}-[(2,4-$ dimethoxyphenyl)methyl]-N-ethynyl-benzamide $3 \mathrm{c}$ as a beige solid (198 $\mathrm{mg}, 75 \%$ yield).

IR (neat): $3244,2131,1660 \mathrm{~cm}^{-1}$

${ }^{1}$ H NMR (500 MHz, DMSO- $\left.d_{6}\right) \delta$ ppm $7.67(\mathrm{~d}, J=7.4 \mathrm{~Hz}, 2 \mathrm{H}), 7.54(\mathrm{t}, J=7.4 \mathrm{~Hz}, 1 \mathrm{H}), 7.47(\mathrm{t}, J=7.4$ $\mathrm{Hz}, 2 \mathrm{H}), 7.18(\mathrm{~d}, J=8.3 \mathrm{~Hz}, 1 \mathrm{H}), 6.6(\mathrm{~d}, J=2.3 \mathrm{~Hz}, 1 \mathrm{H}), 6.53(\mathrm{dd}, J=8.3,2.3 \mathrm{~Hz}, 1 \mathrm{H}), 4.7(\mathrm{~s}, 2 \mathrm{H}), 3.78$ $(\mathrm{s}, 3 \mathrm{H}), 3.77(\mathrm{~s}, 3 \mathrm{H}), 3.65(\mathrm{~s}, 1 \mathrm{H})$

${ }^{13}$ C NMR (125 MHz, DMSO-d $\left.d_{6}\right) \delta$ ppm 170.6, 161, 158.9, 134, 131.8, 130.9, 128.5 (4C), 116, 105, 99, $78.7,64,56,55.6,47.3$

HRMS (EI): calcd. for $\mathrm{C}_{18} \mathrm{H}_{17} \mathrm{~N} \mathrm{O}_{3}\left([\mathrm{M}]^{+}\right)$295.1203, found 295.1201 


\section{N-ethynyl-N-methyl-benzamide 3d}<smiles>C#CN(C)C(=O)c1ccccc1</smiles>

Starting from $\mathrm{N}$-methyl-N-(2-triisopropylsilylethynyl)benzamide $2 \mathrm{~d}(500 \mathrm{mg}, 1.6 \mathrm{mmol}$ ), TBAF $1 \mathrm{M} / \mathrm{THF}\left(2.10 \mathrm{~mL}, 2.1 \mathrm{mmol}, 1.3\right.$ equiv.) under argon, using General procedure 2 and stirring at $0^{\circ} \mathrm{C}$ for $60 \mathrm{~min}$. The crude was purified by silica gel flash column chromatography to afford N-ethynyl-Nmethyl-benzamide $\mathbf{3 d}$ as a yellow oil ( $180 \mathrm{mg}, 71 \%$ yield).

IR (neat): 2138, 1647, $704 \mathrm{~cm}^{-1}$

${ }^{1} \mathrm{H}$ NMR $\left(500 \mathrm{MHz}\right.$, DMSO- $\left.d_{6}\right) \delta \mathrm{ppm} 7.7(\mathrm{~d}, J=7.6 \mathrm{~Hz}, 2 \mathrm{H}), 7.56(\mathrm{t}, J=7.4 \mathrm{~Hz}, 1 \mathrm{H}), 7.48(\mathrm{t}, J=7.6 \mathrm{~Hz}$, $2 \mathrm{H}), 3.85$ (s, $1 \mathrm{H}), 3.25(\mathrm{~s}, 3 \mathrm{H})$

${ }^{13}$ C NMR $\left(125 \mathrm{MHz}\right.$, DMSO- $\left.d_{6}\right) \delta \mathrm{ppm} 170.9,134.2,132,128.6(2 \mathrm{C}), 128.5(2 \mathrm{C}), 62.9,62.9,37.8$

HRMS (ESI): calcd. for $\mathrm{C}_{10} \mathrm{H}_{9} \mathrm{~N} \mathrm{O}\left([\mathrm{M}+\mathrm{H}]^{+}\right)$160.0757, found 160.0757

$\mathrm{mp}: 42.1-45.5^{\circ} \mathrm{C}$

\section{N-benzyl-N-ethynyl-4-methyl-benzamide $3 e$}<smiles>C=CN(Cc1ccccc1)C(=O)c1ccc(C)cc1</smiles>

Starting from N-benzyl-4-methyl-N-(2-triisopropylsilylethynyl)benzamide 2 e (335 $\mathrm{mg}, 0.83 \mathrm{mmol}$ ), TBAF $1 \mathrm{M} / \mathrm{THF}(0.99 \mathrm{~mL}, 1.0 \mathrm{mmol})$ under argon, using General procedure 2 and stirring at $0^{\circ} \mathrm{C}$ for 30 min. The crude was purified by silica gel flash column chromatography to afford N-benzyl-N-ethynyl4-methyl-benzamide $3 e$ as a white solid ( $142 \mathrm{mg}$, $69 \%$ yield).

IR (neat): 3246, 2138, $1664 \mathrm{~cm}^{-1}$

${ }^{1}$ H NMR (400 MHz, DMSO- $\left.d_{6}\right) \delta$ ppm $7.65(\mathrm{~d}, J=8.0 \mathrm{~Hz}, 2 \mathrm{H}), 7.39(\mathrm{~m}, 4 \mathrm{H}), 7.34(\mathrm{~m}, 1 \mathrm{H}), 7.28(\mathrm{~d}, J=$ $7.9 \mathrm{~Hz}, 2 \mathrm{H}), 4.82(\mathrm{~s}, 2 \mathrm{H}), 3.81(\mathrm{~s}, 1 \mathrm{H}), 2.36(\mathrm{~s}, 3 \mathrm{H})$

${ }^{13} \mathrm{C}$ NMR $\left(100 \mathrm{MHz}, \mathrm{DMSO}-d_{6}\right) \delta \mathrm{ppm} 170.5,142.2,136.7,130.9,129.2(4 \mathrm{C}), 128.8(2 \mathrm{C}), 128.4(2 \mathrm{C})$, 128.2, 79.3, 63.9, 52.1, 21

HRMS (ESI): calcd. for $\mathrm{C}_{17} \mathrm{H}_{16} \mathrm{NO}\left([\mathrm{M}+\mathrm{H}]^{+}\right)$250.1226, found 250.1227

$\mathrm{mp}: 57.7-58.3^{\circ} \mathrm{C}$ 


\section{N-benzyl-4-chloro-N-ethynyl-benzamide $3 f$<smiles>C=CN(Cc1ccccc1)C(=O)c1ccc(Cl)cc1</smiles>

Starting from N-benzyl-4-chloro-N-(2-triisopropylsilylethynyl)benzamide $2 \mathrm{f}$ (366 $\mathrm{mg}, 0.86 \mathrm{mmol}$ ), TBAF 1M/THF (1.0 mL, $1.0 \mathrm{mmol})$ under argon, using General procedure 2 and stirring at $0^{\circ} \mathrm{C}$ for 40 $\mathrm{min}$. The crude was purified by silica gel flash column chromatography to afford $\mathrm{N}$-benzyl-4-chloro- $\mathrm{N}$ ethynyl-benzamide $\mathbf{3 f}$ as a white solid ( $145 \mathrm{mg}, 63 \%$ yield).

IR (neat): 3242, 2136, $1670 \mathrm{~cm}^{-1}$

${ }^{1}$ H NMR (400 MHz, DMSO-d $\left.d_{6}\right) \delta$ ppm $7.77(\mathrm{~d}, J=8.3 \mathrm{~Hz}, 2 \mathrm{H}), 7.56(\mathrm{~d}, J=8.4 \mathrm{~Hz}, 2 \mathrm{H}), 7.4 / 7.35(2 \mathrm{~m}, 5$ $\mathrm{H}), 4.83(\mathrm{~s}, 2 \mathrm{H}), 3.87(\mathrm{~s}, 1 \mathrm{H})$

${ }^{13} \mathrm{C}$ NMR $\left(100 \mathrm{MHz}\right.$, DMSO- $\left.d_{6}\right) \delta \mathrm{ppm} 169.7,136.7,136.3,132.4,130.6(2 \mathrm{C}), 129.4(2 \mathrm{C}), 129.0(2 \mathrm{C})$, $128.7(2 \mathrm{C}), 128.4,78.6,64.6,52.3$

HRMS (EI): calcd. for $\mathrm{C}_{16} \mathrm{H}_{12} \mathrm{Cl} \mathrm{N} \mathrm{O}([\mathrm{M}]+)$ 269.0602, found 269.0602

mp: $64-66^{\circ} \mathrm{C}$

\section{N-benzyl-N-ethynyl-4-fluoro-benzamide $3 \mathbf{g}$}<smiles>C=CN(Cc1ccccc1)C(=O)c1ccc(F)cc1</smiles>

Starting from N-benzyl-4-fluoro- $\mathrm{N}$-(2-triisopropylsilylethynyl)benzamide $\mathbf{2 g}(1.72 \mathrm{~g}, 4.20 \mathrm{mmol})$, TBAF $1 \mathrm{M} / \mathrm{THF}(5.0 \mathrm{~mL}, 5.0 \mathrm{mmol})$ under argon, using General procedure 2 and stirring at $0^{\circ} \mathrm{C}$ for $40 \mathrm{~min}$. The crude was purified by trituration in pentane to afford N-benzyl-N-ethynyl-4-fluoro-benzamide $\mathbf{3 g}$ as a white solid (742 $\mathrm{mg}, 70 \%$ yield).

IR (neat): 3242, 2147, 1649, $1604 \mathrm{~cm}^{-1}$

${ }^{1} \mathrm{H}$ NMR (400 MHz, DMSO-d 6 ) $\delta$ ppm $7.88(\mathrm{dd}, J=8.9,5.6 \mathrm{~Hz}, 2 \mathrm{H}), 7.35(\mathrm{~m}, 5 \mathrm{H}), 7.35(\mathrm{t}, J=8.8 \mathrm{~Hz}, 2$ H), $4.86(\mathrm{~s}, 2 \mathrm{H}), 3.9(\mathrm{~s}, 1 \mathrm{H})$

${ }^{13} \mathrm{C}$ NMR $\left(100 \mathrm{MHz}, \mathrm{dmso}-d_{6}\right) \delta \mathrm{ppm} 169.6,164.2(\mathrm{~d}, J=249.5 \mathrm{~Hz}), 136.4,131.6(\mathrm{~d}, J=9.3 \mathrm{~Hz}, 2 \mathrm{C})$, $130.1(\mathrm{~d}, J=3.0 \mathrm{~Hz}), 129.0(2 \mathrm{C}), 128.5(2 \mathrm{C}), 128.3,115.6(\mathrm{~d}, J=22.1 \mathrm{~Hz}, 2 \mathrm{C}), 78.8,64.4,52.3$

${ }^{19}$ F NMR $\left(376 \mathrm{MHz}\right.$, DMSO- $\left.d_{6}\right) \delta \mathrm{ppm}-108.0$

HRMS (EI): calcd. for $\mathrm{C}_{16} \mathrm{H}_{12} \mathrm{~F} \mathrm{~N} \mathrm{O}\left([\mathrm{M}]^{+}\right)$253.0897, found 253.0895

$\mathrm{mp}: 63.7-66.1^{\circ} \mathrm{C}$ 


\section{N-benzyl-N-ethynyl-4-(trifluoromethyl)benzamide $3 \mathrm{~h}$}<smiles>C#CN(Cc1ccccc1)C(=O)c1ccc(C(F)(F)F)cc1</smiles>

Starting from N-benzyl-4-(trifluoromethyl)-N-(2-triisopropylsilylethynyl)benzamide $\mathbf{2 h}$ (355 mg, 0.77 $\mathrm{mmol}), \operatorname{TBAF} 1 \mathrm{M} / \mathrm{THF}(0.93 \mathrm{~mL}, 0.9 \mathrm{mmol})$ under argon, using General procedure 2 and stirring at $0^{\circ} \mathrm{C}$ for $40 \mathrm{~min}$. The crude was purified by silica gel flash column chromatography to afford N-benzyl$\mathrm{N}$-ethynyl-4-(trifluoromethyl)benzamide $3 \mathrm{~h}$ as a white solid (139 $\mathrm{mg}, 59 \%$ yield).

IR (neat): 3238, 2135, 1664, 1319, 1136, $1124 \mathrm{~cm}^{-1}$

${ }^{1} \mathrm{H}$ NMR $\left(400 \mathrm{MHz}\right.$, DMSO- $\left.d_{6}\right) \delta \mathrm{ppm} 7.93(\mathrm{~d}, J=7.9 \mathrm{~Hz}, 2 \mathrm{H}), 7.87(\mathrm{~d}, J=7.9 \mathrm{~Hz}, 2 \mathrm{H}), 7.4(\mathrm{~m}, 4 \mathrm{H})$, $7.35(\mathrm{~m}, 1 \mathrm{H}), 4.85(\mathrm{~s}, 2 \mathrm{H}), 3.88(\mathrm{~s}, 1 \mathrm{H})$

${ }^{13} \mathrm{C}$ NMR (100 MHz, DMSO-d $\left.d_{6}\right) \delta$ ppm 169.6, 137.8, 136.2, 131.6 (q, J = 32.0 Hz), 129.4 (2C), 129.1 (2C), $128.5(2 \mathrm{C}), 128.4,125.6(q, J=3.7 \mathrm{~Hz}, 2 \mathrm{C}), 124.0$ (q, J = 271.9 Hz), 78.3, 64.9, 52.2

${ }^{19}$ F NMR $\left(376 \mathrm{MHz}, \mathrm{DMSO}-d_{6}\right) \delta \mathrm{ppm}-61.4$

HRMS (EI): calcd. for $\mathrm{C}_{17} \mathrm{H}_{12} \mathrm{~F}_{3} \mathrm{NO}\left([\mathrm{M}]^{+}\right)$303.0866, found 303.0869

$\mathrm{mp}: 67-69^{\circ} \mathrm{C}$

\section{N-benzyl-4-tert-butyl-N-ethynyl-benzamide $3 \mathbf{i}$}<smiles>C=CN(Cc1ccccc1)C(=O)c1ccc(C(C)(C)C)cc1C</smiles>

Starting from N-benzyl-4-tert-butyl-N-(2-triisopropylsilylethynyl)benzamide $\mathbf{2 i}$ ( $215 \mathrm{mg}, 0.48 \mathrm{mmol}$ ), TBAF $1 \mathrm{M} / \mathrm{THF}(0.58 \mathrm{~mL}, 0.6 \mathrm{mmol})$ under argon, using General procedure 2 and stirring at $0^{\circ} \mathrm{C}$ for 40 min. The crude was purified by silica gel flash column chromatography to afford N-benzyl-4-tertbutyl-N-ethynyl-benzamide $3 \mathbf{i}$ as a colorless oil ( $82 \mathrm{mg}, 59 \%$ yield).

IR (neat): $3230,2137,1672 \mathrm{~cm}^{-1}$

${ }^{1} \mathrm{H}$ NMR $\left(400 \mathrm{MHz}\right.$, DMSO- $\left.d_{6}\right) \delta \mathrm{ppm} 7.71(\mathrm{~d}, J=8.2 \mathrm{~Hz}, 2 \mathrm{H}), 7.5(\mathrm{~d}, J=8.2 \mathrm{~Hz}, 2 \mathrm{H}), 7.39(\mathrm{~m}, 4 \mathrm{H})$, $7.34(\mathrm{~m}, 1 \mathrm{H}), 4.82(\mathrm{~s}, 2 \mathrm{H}), 3.85(\mathrm{~s}, 1 \mathrm{H}), 1.3(\mathrm{~s}, 9 \mathrm{H})$

${ }^{13} \mathrm{C}$ NMR $\left(125 \mathrm{MHz}, \mathrm{DMSO}-d_{6}\right) \delta \mathrm{ppm} 170.7,155.1,136.9,131.1,129.0$ (2C), $128.8(2 \mathrm{C}), 128.4(2 \mathrm{C})$, $128.2,125.3$ (2C), 79.4, 64, 52.3, 35.3, 31.4 (3C)

HRMS (EI): calcd. for $\mathrm{C}_{20} \mathrm{H}_{21} \mathrm{~N} \mathrm{O}\left([\mathrm{M}]^{+}\right)$291.1618, found 291.1608 


\section{N-benzyl-N-ethynyl-4-nitro-benzamide 3j}<smiles>C#CN(Cc1ccccc1)C(=O)c1ccc([N+](=O)[O-])cc1</smiles>

Starting from N-benzyl-4-nitro-N-(2-triisopropylsilylethynyl)benzamide $2 \mathrm{j}$ ( $408 \mathrm{mg}, 0.93 \mathrm{mmol}$ ), TBAF 1M/THF (1.1 mL, $1.1 \mathrm{mmol}$ ) under argon, using General procedure 2 and stirring at $0^{\circ} \mathrm{C}$ for $25 \mathrm{~min}$. The crude was purified by silica gel flash column chromatography to afford N-benzyl-N-ethynyl-4nitro-benzamide $\mathbf{3} \mathbf{j}$ as a beige solid ( $170 \mathrm{mg}, 65 \%$ yield).

IR (neat): $3236,2133,1668,1602,1523,1350 \mathrm{~cm}^{-1}$

${ }^{1}$ H NMR $(400 \mathrm{MHz}$, DMSO-d 6 ) $\delta$ ppm $8.32(\mathrm{~d}, J=8.5 \mathrm{~Hz}, 2 \mathrm{H}), 7.98(\mathrm{~d}, J=8.6 \mathrm{~Hz}, 2 \mathrm{H}), 7.41(\mathrm{~m}, 4 \mathrm{H})$, $7.35(\mathrm{~m}, 1 \mathrm{H}), 4.86(\mathrm{~s}, 2 \mathrm{H}), 3.9(\mathrm{~s}, 1 \mathrm{H})$

${ }^{13} \mathrm{C}$ NMR (125 MHz, DMSO- $\left.d_{6}\right) \delta$ ppm 169.7, 149.3, 139.8, 136.1, $130.0(2 \mathrm{C}), 129.1(2 \mathrm{C}), 128.6(2 \mathrm{C})$, $128.4,123.9(2 \mathrm{C}), 78.6,65.1,52.3$

HRMS (EI): calcd. for $\mathrm{C}_{16} \mathrm{H}_{12} \mathrm{~N}_{2} \mathrm{O}_{3}\left([\mathrm{M}]^{+}\right)$280.0842, found 280.083

$\mathrm{mp}: 42.9-44.4^{\circ} \mathrm{C}$

\section{N-benzyl-4-cyano-N-ethynyl-benzamide 3k}<smiles>C#CN(Cc1ccccc1)C(=O)c1ccc(C#N)cc1</smiles>

Starting from N-benzyl-4-cyano-N-(2-triisopropylsilylethynyl)benzamide $2 \mathrm{k}$ (239 $\mathrm{mg}, 0.57 \mathrm{mmol}$ ), TBAF 1M/THF (0.69 mL, $0.7 \mathrm{mmol})$ under argon, using General procedure 2 and stirring at $0^{\circ} \mathrm{C}$ for 40 min. The crude was purified by trituration in pentane to afford N-benzyl-4-cyano- $\mathrm{N}$-ethynylbenzamide $3 \mathbf{k}$ as a white solid (116 $\mathrm{mg}, 78 \%$ yield).

IR (neat): 3232, 2235, 2137, 1670, $1609 \mathrm{~cm}^{-1}$

${ }^{1} \mathrm{H}$ NMR $\left(400 \mathrm{MHz}, \mathrm{DMSO}-d_{6}\right) \delta \mathrm{ppm} 8.0(\mathrm{~d}, J=8.2 \mathrm{~Hz}, 2 \mathrm{H}), 7.9(\mathrm{~d}, J=8.2 \mathrm{~Hz}, 2 \mathrm{H}), 7.35(\mathrm{~m}, 5 \mathrm{H}), 4.86$ (s, $2 \mathrm{H}), 3.9(\mathrm{~s}, 1 \mathrm{H})$

${ }^{13} \mathrm{C}$ NMR $\left(100 \mathrm{MHz}\right.$, DMSO-d $\left.d_{6}\right) \delta \mathrm{ppm} 169.5,138.1,135.9,132.8$ (2C), 129.3 (2C), 129.0 (2C), 128.5 (2C), 128.4, 118.6, 114.2, 78.1, 65, 52.2

HRMS (EI): calcd. for $\mathrm{C}_{17} \mathrm{H}_{12} \mathrm{~N}_{2} \mathrm{O}$ ([M] $\left.]^{+}\right) 260.0944$, found 260.0929

$\mathrm{mp}: 114-118^{\circ} \mathrm{C}$ 


\section{methyl 4-[benzyl(ethynyl)carbamoyl]benzoate 3I}<smiles>C#CN(Cc1ccccc1)C(=O)c1ccc(C(=O)OC)cc1</smiles>

Starting from methyl 4-[benzyl(2-triisopropylsilylethynyl)carbamoyl]benzoate 2l (315 mg, 0.70 $\mathrm{mmol}), \operatorname{TBAF} 1 \mathrm{M} / \mathrm{THF}(0.8 \mathrm{~mL}, 0.8 \mathrm{mmol})$ under argon, using General procedure 2 and stirring at $0^{\circ} \mathrm{C}$ for $40 \mathrm{~min}$. The crude was purified by silica gel flash column chromatography to afford methyl 4[benzyl(ethynyl)carbamoyl]benzoate $\mathbf{3 l}$ as a yellow solid (121 mg, $59 \%$ yield).

IR (neat): 3230, 2137, 1718, $1674 \mathrm{~cm}^{-1}$

${ }^{1} \mathbf{H}$ NMR $\left(400 \mathrm{MHz}\right.$, DMSO- $\left.d_{6}\right) \delta \mathrm{ppm} 8.04(\mathrm{~d}, J=8.2 \mathrm{~Hz}, 2 \mathrm{H}), 7.84(\mathrm{~d}, J=8.3 \mathrm{~Hz}, 2 \mathrm{H}), 7.4(\mathrm{~m}, 4 \mathrm{H})$, $7.35(\mathrm{~m}, 1 \mathrm{H}), 4.85(\mathrm{~s}, 2 \mathrm{H}), 3.89(\mathrm{~s}, 3 \mathrm{H}), 3.85(\mathrm{~s}, 1 \mathrm{H})$

${ }^{13} \mathrm{C}$ NMR $\left(100 \mathrm{MHz}, \mathrm{DMSO}-d_{6}\right) \delta \mathrm{ppm} 170.9,166.6,138.9,136.2,132.3,129.4$ (2C), 129.0 (2C), 128.9 (2C), $128.5(2 \mathrm{C}), 128.2,78.2,64.7,52.9,52.3$

HRMS (EI): calcd. for $\mathrm{C}_{18} \mathrm{H}_{15} \mathrm{~N} \mathrm{O}_{3}\left([\mathrm{M}]^{+}\right)$293.1046, found 293.1034

$m p: 93.3-95.3^{\circ} \mathrm{C}$

\section{N-benzyl-N-ethynyl-pyridine-4-carboxamide 3m}<smiles>C#CN(Cc1ccccc1)C(=O)c1ccncc1</smiles>

Starting from $\mathrm{N}$-benzyl-N-(2-triisopropylsilylethynyl)pyridine-4-carboxamide $\mathbf{2 m}(514 \mathrm{mg}, 1.31$ $\mathrm{mmol})$, TBAF $1 \mathrm{M} / \mathrm{THF}(1.6 \mathrm{~mL}, 1.6 \mathrm{mmol})$ under argon, using General procedure 2 and stirring at $0^{\circ} \mathrm{C}$ for $30 \mathrm{~min}$. The crude was purified by trituration in pentane to afford $\mathrm{N}$-benzyl-N-ethynyl-pyridine-4carboxamide $3 \mathrm{~m}$ as a white solid (179 $\mathrm{mg}, 58 \%$ yield).

IR (neat): 3233, 2139, 1674, 831, 739, $696 \mathrm{~cm}^{-1}$

${ }^{1} \mathrm{H}$ NMR $\left(400 \mathrm{MHz}\right.$, DMSO- $\left.d_{6}\right) \delta \mathrm{ppm} 8.74(\mathrm{~d}, J=4.9 \mathrm{~Hz}, 2 \mathrm{H}), 7.65(\mathrm{~d}, J=4.7 \mathrm{~Hz}, 2 \mathrm{H}), 7.4(\mathrm{~m}, 4 \mathrm{H})$, $7.35(\mathrm{~m}, 1 \mathrm{H}), 4.84(\mathrm{~s}, 2 \mathrm{H}), 3.9(\mathrm{~s}, 1 \mathrm{H})$

${ }^{13} \mathrm{C}$ NMR (100 MHz, DMSO- $d_{6}$ ) $\delta$ ppm 169.2, 150.2 (2C), 141.4, 136.2, 129.1 (2C), 128.5 (2C), 128.4, $122.1(2 \mathrm{C}), 77.9,64.8,51.6$

HRMS (ESI): calcd. for $\mathrm{C}_{15} \mathrm{H}_{13} \mathrm{~N}_{2} \mathrm{O}\left([\mathrm{M}+\mathrm{H}]^{+}\right)$237.1022, found 237.1022

mp: $62.5-64.5^{\circ} \mathrm{C}$ 


\section{N-benzyl-N-ethynyl-1,3-benzothiazole-6-carboxamide 3n}<smiles>C#CN(Cc1ccccc1)C(=O)c1ccc2c(c1)SCN2</smiles>

Starting from N-benzyl-N-(2-triisopropylsilylethynyl)-1,3-benzothiazole-6-carboxamide 2 n (401 mg, $0.89 \mathrm{mmol})$, TBAF $1 \mathrm{M} / \mathrm{THF}(1.07 \mathrm{~mL}, 1.07 \mathrm{mmol})$ under argon, using General procedure 2 and stirring at $0^{\circ} \mathrm{C}$ for $25 \mathrm{~min}$. The crude was purified by silica gel flash column chromatography to N-benzyl-Nethynyl-1,3-benzothiazole-6-carboxamide $3 \mathrm{n}$ as a yellow solid (178 $\mathrm{mg}$, $68 \%$ yield).

IR (neat): 3234, 2137, $1668 \mathrm{~cm}^{-1}$

${ }^{1} \mathrm{H}$ NMR $\left(400 \mathrm{MHz}\right.$, DMSO- $\left.d_{6}\right) \delta \mathrm{ppm} 9.56(\mathrm{~s}, 1 \mathrm{H}), 8.62(\mathrm{~d}, J=1.5 \mathrm{~Hz}, 1 \mathrm{H}), 8.17(\mathrm{~d}, J=8.5 \mathrm{~Hz}, 1 \mathrm{H})$, $7.88(\mathrm{dd}, J=8.4,1.7 \mathrm{~Hz}, 1 \mathrm{H}), 7.42(\mathrm{~m}, 4 \mathrm{H}), 7.36(\mathrm{~m}, 1 \mathrm{H}), 4.88(\mathrm{~s}, 2 \mathrm{H}), 3.84(\mathrm{~s}, 1 \mathrm{H})$

${ }^{13} \mathrm{C}$ NMR $\left(100 \mathrm{MHz}\right.$, DMSO-d $\left.d_{6}\right) \delta \mathrm{ppm} 170.3,160,155,136.4,133.8,130.7,129.1(2 \mathrm{C}), 128.5(2 \mathrm{C})$, $128.3,126.6,123.7,122.9,78.7,64.3,52.3$

HRMS (EI): calcd. for $\mathrm{C}_{17} \mathrm{H}_{12} \mathrm{~N}_{2} \mathrm{O}$ S ([M] $\left.]^{+}\right)$292.0665, found 292.0657

$\mathrm{mp}: 125.7-127.7^{\circ} \mathrm{C}$

\section{$\mathrm{N}$-benzyl-N-ethynyl-3,5-difluoro-benzamide 30}<smiles>C=CN(Cc1ccccc1)C(=O)c1cc(F)cc(F)c1</smiles>

Starting from N-benzyl-3,5-difluoro- $\mathrm{N}$-(2-triisopropylsilylethynyl)benzamide 20 (333 $\mathrm{mg}, 0.78 \mathrm{mmol}$ ), TBAF 1M/THF $(0.93 \mathrm{~mL}, 0.9 \mathrm{mmol})$ under argon, using General procedure 2 and stirring at $0^{\circ} \mathrm{C}$ for 40 min. The crude was purified by silica gel flash column chromatography to afford $\mathrm{N}$-benzyl-N-ethynyl3,5-difluoro-benzamide $\mathbf{3 0}$ as a white solid (73 mg, $35 \%$ yield).

IR (neat): 3247, 2137, 1674, 1622, $1591 \mathrm{~cm}^{-1}$

${ }^{1}{ }_{\text {H NMR }}(400 \mathrm{MHz}$, DMSO-d 6 ) $\delta$ ppm $7.45(\mathrm{~m}, 3 \mathrm{H}), 7.4(\mathrm{~m}, 4 \mathrm{H}), 7.35(\mathrm{~m}, 1 \mathrm{H}), 4.83(\mathrm{~s}, 2 \mathrm{H}), 3.92(\mathrm{~s}, 1$ H)

${ }^{13} \mathrm{C}$ NMR $\left(100 \mathrm{MHz}, \mathrm{DMSO}-d_{6}\right) \delta \mathrm{ppm} 168.3,162.3(\mathrm{dd}, J=248.2,12.7 \mathrm{~Hz}, 2 \mathrm{C}), 137.3(\mathrm{t}, J=9.2 \mathrm{~Hz})$, $136.1,129.0(2 \mathrm{C}), 128.5(2 \mathrm{C}), 128.2,112.2(\mathrm{~d}, J=27.3 \mathrm{~Hz}, 2 \mathrm{C}), 107.2(\mathrm{t}, J=25.6 \mathrm{~Hz}), 78.1,65.1,52.5$

${ }^{19}$ F NMR $\left(376 \mathrm{MHz}\right.$, DMSO- $\left.d_{6}\right) \delta \mathrm{ppm}-108.8$

HRMS (EI): calcd. for $\mathrm{C}_{16} \mathrm{H}_{11} \mathrm{~F}_{2} \mathrm{~N} O$ ([M] $\left.]^{+}\right)$271.0803, found 271.0775

$\mathrm{mp}: 87.7-91.4^{\circ} \mathrm{C}$ 


\section{N-benzyl-N-ethynyl-2-methoxy-benzamide 3p}<smiles>C=CN(Cc1ccccc1)C(=O)c1ccccc1OC</smiles>

Starting from N-benzyl-2-methoxy-N-(2-triisopropylsilylethynyl)benzamide $2 \mathrm{p}$ (447 mg, $1.06 \mathrm{mmol}$ ), TBAF 1M/THF (1.27 mL, $1.3 \mathrm{mmol})$ under argon, using General procedure 2 and stirring at $0^{\circ} \mathrm{C}$ for 40 min. The crude was purified by trituration in pentane to afford N-benzyl-N-ethynyl-2-methoxybenzamide $3 p$ as a white solid ( $200 \mathrm{mg}, 71 \%$ yield).

IR (neat): 3245, 2146, 1672, 751, $703 \mathrm{~cm}^{-1}$

${ }^{1} \mathrm{H}$ NMR $\left(400 \mathrm{MHz}\right.$, DMSO- $\left.d_{6}\right) \delta \mathrm{ppm} 7.45$ (ddd, $\left.J=8.0,7.7,1.7 \mathrm{~Hz}, 1 \mathrm{H}\right), 7.38(\mathrm{~m}, 5 \mathrm{H}), 7.31(\mathrm{dd}, J=$ 7.8, 1.6 Hz, $1 \mathrm{H}), 7.12(\mathrm{~d}, J=8.2 \mathrm{~Hz}, 1 \mathrm{H}), 7.01(\mathrm{t}, J=7.5 \mathrm{~Hz}, 1 \mathrm{H}), 4.82(\mathrm{~s}, 2 \mathrm{H}), 3.82(\mathrm{~s}, 3 \mathrm{H}), 3.55(\mathrm{bs}, 1$ H)

${ }^{13} \mathrm{C}$ NMR $\left(100 \mathrm{MHz}\right.$, DMSO- $\left.d_{6}\right) \delta \mathrm{ppm} 170.1,156.4,136.5,132,128.8(2 \mathrm{C}), 127.9(2 \mathrm{C}), 128.3,127.9$, $124.8,120.8,112.1,78.2,62.8,56.3,51$

HRMS (EI): calcd. for $\mathrm{C}_{17} \mathrm{H}_{15} \mathrm{~N} \mathrm{O}_{2}\left([\mathrm{M}]^{+}\right)$265.1097, found 265.1096

\section{N-ethynyl-N-(3-methoxypropyl)benzamide 3q}<smiles>C=CN(CCCOC)C(=O)c1ccccc1</smiles>

Starting from $\mathrm{N}$-(3-methoxypropyl)- $\mathrm{N}$-(2-triisopropylsilylethynyl)benzamide $2 \mathrm{q}$ (189 $\mathrm{mg}, 0.51 \mathrm{mmol}$ ), TBAF $1 \mathrm{M} / \mathrm{THF}(0.61 \mathrm{~mL}, 0.6 \mathrm{mmol})$ under argon, using General procedure 2 and stirring at $0^{\circ} \mathrm{C}$ for $1 \mathrm{~h} 30$. The crude was purified by silica gel flash column chromatography to afford N-ethynyl-N-(3methoxypropyl)benzamide $\mathbf{3 q}$ as a yellow oil ( $86 \mathrm{mg}$, $78 \%$ yield).

IR (neat): 2136, 1671, $1601 \mathrm{~cm}^{-1}$

${ }^{1} \mathrm{H}$ NMR (500 MHz, dmso-d6) $\delta$ ppm 7.68 (d, J = 7.5 Hz, $\left.2 \mathrm{H}\right), 7.54$ (t, J = 7.3 Hz, $1 \mathrm{H}$ ), 7.47 (t, J = $7.5 \mathrm{~Hz}$, $2 \mathrm{H}$ ), $3.85(\mathrm{~s}, 1 \mathrm{H}), 3.69(\mathrm{t}, J=6.9 \mathrm{~Hz}, 2 \mathrm{H}), 3.42(\mathrm{t}, J=6.2 \mathrm{~Hz}, 2 \mathrm{H}), 3.24(\mathrm{~s}, 3 \mathrm{H}), 1.81$ (quint, $J=6.6 \mathrm{~Hz}$, $2 \mathrm{H})$

${ }^{13} \mathrm{C}$ NMR (100 MHz, dmso-d6) $\delta$ ppm 170.5, 134.2, 131.7 (2C), 128.3 (2C), 128.2, 78.7, 69.5, 63.7, $58.5,46.5,27.7$

HRMS (ESI): calcd. for $\mathrm{C}_{13} \mathrm{H}_{15} \mathrm{~N} \mathrm{O}_{2}\left([\mathrm{M}+\mathrm{H}]^{+}\right)$218.1181, found 218.1176 


\section{General procedure 3 for the cascade processes: Synthesis of isoindolinones 4a-q}

In a $10 \mathrm{~mL}$ Schlenk tube (prealably dried under vacuum) under argon, were loaded the ynamide 3 (1 equiv.), Togni I reagent (3,3-dimethyl-1-(trifluoromethyl)-1lambda3,2-benziodoxole) (1.2 equiv.) and $\operatorname{Ir}(\mathrm{ppy})_{3}(0.025$ equiv.). The tube was degassed with 3 vacuum/argon cycles. Anhydrous acetonitrile on molecular sieves $(0.1 \mathrm{M})$ followed by dichloromethane $(5 \% \mathrm{v} / \mathrm{v})$ were added under argon. The reaction mixture was freeze pumped once to deoxygenate the solvent. The reaction mixture was irradiated under blue LED at $27^{\circ} \mathrm{C}$ approximately for the given amount of time. After completion of the reaction, the reaction mixture was concentrated under vacuum. The crude was purified by silica gel flash column chromatography (solid deposit on Celite, gradient eluent using a mixture of EtOAc $2 \% / \mathrm{CH}_{2} \mathrm{Cl}_{2} 1 \%$ in heptane). The separation was tricky, the by-product of Togni I was eluted close to the expected product.

\section{(3E/Z)-2-benzyl-3-(2,2,2-trifluoroethylidene)isoindolin-1-one 4a}<smiles>O=C1c2ccccc2/C(=C\C(F)(F)F)N1Cc1ccccc1</smiles>

In a $25 \mathrm{~mL}$ Schlenk tube, starting from N-benzyl-N-ethynyl-benzamide $3 a(250 \mathrm{mg}, 1.06 \mathrm{mmol}$ ), Togni I reagent $(421 \mathrm{mg}, 1.3 \mathrm{mmol})$ and $\operatorname{Ir}(\mathrm{ppy})_{3}(17.5 \mathrm{mg}, 0.03 \mathrm{mmol})$, using General procedure 3 and stirring at $27^{\circ} \mathrm{C}$ for $5 \mathrm{~h}$. The crude was purified by silica gel flash column chromatography to afford a mixture of (3E/Z)-2-benzyl-3-(2,2,2-trifluoroethylidene)isoindolin-1-one 4a as a pale yellow oil (213 $\mathrm{mg}, 64 \%$ yield) (E/Z mixture $81 / 19$ ).

(3E)-2-benzyl-3-(2,2,2-trifluoroethylidene)isoindolin-1-one $4 a E$

IR (neat): $1732,1656,1319,1109 \mathrm{~cm}^{-1}$ (mixture 81/19 E/Z)

${ }^{1}$ H NMR $\left(400 \mathrm{MHz}\right.$, DMSO- $\left.d_{6}\right) \delta \mathrm{ppm} 7.96 / 7.94(2 \mathrm{~d}, J=8.2 \mathrm{~Hz}, 2 \mathrm{H}), 7.85 / 7.77(2 \mathrm{t}, J=7.6 \mathrm{~Hz}, 2 \mathrm{H})$, $7.35(\mathrm{~m}, 3 \mathrm{H}), 7.27(\mathrm{~m}, 2 \mathrm{H}), 5.93(\mathrm{q}, J=9.5 \mathrm{~Hz}, 1 \mathrm{H}), 5.05(\mathrm{~s}, 2 \mathrm{H})$

${ }^{13} \mathrm{C}$ NMR (100 MHz, DMSO- $d_{6}$ ) $\delta$ ppm 166.4, 144.5 (q, $\left.J=6.3 \mathrm{~Hz}\right), 136,134.1,132.4,131.9(2 \mathrm{C}), 129.2$ (2C), 127.5, $127.4(2 \mathrm{C}), 125.5,124.8(q, J=268.6 \mathrm{~Hz}), 124.1,95.9(q, J=38.0 \mathrm{~Hz}), 42.6$

$167.7,141.4,136.9,136.5,132.2,129 / 128.4,125.7,123.9,122.2,92.2,44.7$

${ }^{19}$ F NMR $\left(376 \mathrm{MHz}\right.$, DMSO- $\left.d_{6}\right) \delta \mathrm{ppm}-51.87$

HRMS (ESI): calcd. for $\mathrm{C}_{17} \mathrm{H}_{12} \mathrm{~F}_{3} \mathrm{~N} \mathrm{O}\left([\mathrm{M}+\mathrm{H}]^{+}\right)$304.0944, found 304.0942

mp: $94.6-101.9^{\circ} \mathrm{C}$

(3Z)-2-benzyl-3-(2,2,2-trifluoroethylidene)isoindolin-1-one $4 a Z$

${ }^{1}$ H NMR (400 MHz, DMSO-d $\left.)_{6}\right) \delta$ ppm $8.23(\mathrm{~d}, J=7.8 \mathrm{~Hz}, 1 \mathrm{H}), 7.89(\mathrm{~d}, J=7.6 \mathrm{~Hz}, 1 \mathrm{H}), 7.73$ (t, $J=7.5$ $\mathrm{Hz}, 2 \mathrm{H}), 7.31(\mathrm{~m}, 3 \mathrm{H}), 7.07(\mathrm{~d}, J=7.5 \mathrm{~Hz}, 2 \mathrm{H}), 6.37$ (q, J = $10.1 \mathrm{~Hz}, 1 \mathrm{H}), 5.12(\mathrm{~s}, 2 \mathrm{H})$

${ }^{13} \mathrm{C}$ NMR $\left(100 \mathrm{MHz}\right.$, DMSO- $\left.d_{6}\right) \delta \mathrm{ppm} 167.7,141.4$ (q, $\left.J=6.6 \mathrm{~Hz}\right), 136.9(2 \mathrm{C}), 136.5,132.2(2 \mathrm{C}), 129$ (2C), 128.4, $125.7(2 \mathrm{C}), 123.9,122.9$ ( $\mathrm{q}, J=268.6 \mathrm{~Hz}), 122.2,92.2(\mathrm{q}, J=38.0 \mathrm{~Hz}), 44.7$ 
${ }^{19}$ F NMR $\left(376 \mathrm{MHz}\right.$, DMSO- $\left.d_{6}\right) \delta \mathrm{ppm}-48.85$

\section{(3E/Z)-2-[(4-methoxyphenyl)methyl]-3-(2,2,2-trifluoroethylidene)isoindolin-1-one} 4b<smiles>COc1ccc(CN2C(=O)c3ccccc3/C2=C\C(F)(F)F)cc1</smiles>

Starting from N-[(2,4-dimethoxyphenyl)methyl]-N-ethynyl-benzamide $\mathbf{3 b}$ (106 mg, $0.4 \mathrm{mmol}$ ), Togni I reagent $(158 \mathrm{mg}, 0.48 \mathrm{mmol})$ and $\operatorname{Ir}(\mathrm{ppy})_{3}(6.6 \mathrm{mg}, 0.01 \mathrm{mmol})$, using General procedure 3 and stirring at $27^{\circ} \mathrm{C}$ for $5.5 \mathrm{~h}$. The crude was purified by silica gel flash column chromatography to afford a mixture of (3E/Z)-2-[(4-methoxyphenyl)methyl]-3-(2,2,2-trifluoroethylidene)isoindolin-1-one $\mathbf{4 b}$ as a pale yellow oil ( $78 \mathrm{mg}, 59 \%$ yield) (E/Z mixture 60/40).

(3E)-2-[(4-methoxyphenyl)methyl]-3-(2,2,2-trifluoroethylidene)isoindolin-1-one $4 b E$ IR (neat): 1728, 1655, 1244, $1086 \mathrm{~cm}^{-1}$ (mixture 60/40 E/Z)

${ }^{1} \mathbf{H}$ NMR $\left(500 \mathrm{MHz}\right.$, DMSO- $\left.d_{6}\right) \delta \mathrm{ppm} 7.95(\mathrm{~d}, J=8.1 \mathrm{~Hz}, 1 \mathrm{H}), 7.93(\mathrm{~d}, J=8.1 \mathrm{~Hz}, 1 \mathrm{H}), 7.84(\mathrm{t}, J=7.7$ $\mathrm{Hz}, 1 \mathrm{H}), 7.76(\mathrm{t}, J=7.5 \mathrm{~Hz}, 1 \mathrm{H}), 7.23(\mathrm{~d}, J=8.3 \mathrm{~Hz}, 2 \mathrm{H}), 6.91(\mathrm{~d}, J=8.5 \mathrm{~Hz}, 2 \mathrm{H}), 5.95(\mathrm{q}, J=9.4 \mathrm{~Hz}, 1$ $\mathrm{H}), 4.97(\mathrm{~s}, 2 \mathrm{H}), 3.73(\mathrm{~s}, 3 \mathrm{H})$

${ }^{13} \mathrm{C}$ NMR (125 MHz, DMSO- $\left.d_{6}\right) \delta \mathrm{ppm} 166.5,159.1,144.4(\mathrm{q}, J=6.3 \mathrm{~Hz}), 134,132.1,132.1,130.1$, $128.7(2 \mathrm{C}), 128.3,123.7 / 125.8$ ( $\mathrm{q}, J=267.9 \mathrm{~Hz}), 124.3,124,114.3(2 \mathrm{C}), 95.9$ ( $\mathrm{q}, J=37.9 \mathrm{~Hz}$ ), 55.4, 42.1

${ }^{19}$ F NMR (376 MHz, DMSO- $\left.d_{6}\right) \delta \mathrm{ppm}-51.8$

HRMS (ESI): calcd. for $\mathrm{C}_{18} \mathrm{H}_{15} \mathrm{~F}_{3} \mathrm{~N} \mathrm{O}_{2}\left([\mathrm{M}+\mathrm{H}]^{+}\right)$334.1049, found 334.105

mp: $76.5-84.2^{\circ} \mathrm{C}$ (mixture $60 / 40 \mathrm{E} / \mathrm{Z}$ )

(3Z)-2-[(4-methoxyphenyl)methyl]-3-(2,2,2-trifluoroethylidene)isoindolin-1-one $4 b Z$

${ }^{1} \mathrm{H}$ NMR $\left(400 \mathrm{MHz}\right.$, DMSO- $\left.d_{6}\right) \delta \mathrm{ppm} 8.22(\mathrm{~d}, J=7.8 \mathrm{~Hz}, 1 \mathrm{H}), 7.87(\mathrm{~d}, J=7.5 \mathrm{~Hz}, 1 \mathrm{H}), 7.81(\mathrm{t}, J=7.9$ $\mathrm{Hz}, 1 \mathrm{H}), 7.73(\mathrm{t}, J=7.5 \mathrm{~Hz}, 1 \mathrm{H}), 7(\mathrm{~d}, J=8.5 \mathrm{~Hz}, 2 \mathrm{H}), 6.86(\mathrm{~d}, J=8.7 \mathrm{~Hz}, 2 \mathrm{H}), 6.35(\mathrm{q}, J=10.04 \mathrm{~Hz}, 1$ $\mathrm{H}), 5.04(\mathrm{~s}, 2 \mathrm{H}), 3.71(\mathrm{~s}, 3 \mathrm{H})$

${ }^{13} \mathrm{C}$ NMR $\left(100 \mathrm{MHz}\right.$, DMSO- $\left.d_{6}\right) \delta \mathrm{ppm} 168.4,158.5,142$ (q, $\left.J=6.5 \mathrm{~Hz}\right), 137.3,134.2,132,128.8,127.1$, $127(2 \mathrm{C}), 123.8(q, J=266.9 \mathrm{~Hz}), 123.8,122,114.4(2 \mathrm{C}), 91.9(q, J=37.9 \mathrm{~Hz}), 55.5,44.2$

${ }^{19}$ F NMR $\left(376 \mathrm{MHz}\right.$, DMSO- $\left.d_{6}\right) \delta \mathrm{ppm}-48.8$ 


\section{(3E/Z)-2-[(2,4-dimethoxyphenyl)methyl]-3-(2,2,2-trifluoroethylidene)isoindolin-1-} one 4c<smiles>COc1ccc(CN2C(=O)c3ccccc3/C2=C\C(F)(F)F)c(OC)c1</smiles>

Starting from N-[(2,4-dimethoxyphenyl)methyl]-N-ethynyl-benzamide $3 \mathrm{c}(82 \mathrm{mg}, 0.28 \mathrm{mmol})$, Togni I reagent $(110 \mathrm{mg}, 0.33 \mathrm{mmol})$ and $\operatorname{Ir}(\mathrm{ppy})_{3}(4.6 \mathrm{mg}, 0.01 \mathrm{mmol})$, using General procedure 3 and stirring at $27^{\circ} \mathrm{C}$ for $3 \mathrm{~h}$. The crude was purified by silica gel flash column chromatography to afford (3E)-2-[(2,4-dimethoxyphenyl)methyl]-3-(2,2,2-trifluoroethylidene)isoindolin-1-one 4cE as a white solid (39 mg) and (3Z)-2-[(2,4-dimethoxyphenyl)methyl]-3-(2,2,2-trifluoroethylidene)isoindolin-1-one $4 c Z$ as a white solid (18 $\mathrm{mg})$ (E/Z ratio 70/30, $57 \%$ overall yield).

(3E)-2-[(2,4-dimethoxyphenyl)methyl]-3-(2,2,2-trifluoroethylidene)isoindolin-1-one 4cE IR (neat): 1723, 1659, 1614, 1258, $1093 \mathrm{~cm}^{-1}$

${ }^{1} \mathbf{H}$ NMR $\left(500 \mathrm{MHz}\right.$, DMSO- $\left.d_{6}\right) \delta \mathrm{ppm} 7.97 / 7.93(2 \mathrm{~d}, J=7.6 \mathrm{~Hz}, 2 \mathrm{H}), 7.86 / 7.79(2 \mathrm{t}, J=7.6 \mathrm{~Hz}, 2 \mathrm{H}), 6.9$ $(\mathrm{d}, J=8.5 \mathrm{~Hz}, 1 \mathrm{H}), 6.63(\mathrm{~d}, J=2.4 \mathrm{~Hz}, 1 \mathrm{H}), 6.5(\mathrm{dd}, J=8.4 \mathrm{~Hz}, 1 \mathrm{H}), 5.85(\mathrm{q}, J=9.5 \mathrm{~Hz}, 1 \mathrm{H}), 4.9(\mathrm{~s}, 2$ $\mathrm{H}), 3.85 / 3.75(\mathrm{~s}, 6 \mathrm{H})$

${ }^{13}$ C NMR $\left(125 \mathrm{MHz}\right.$, DMSO- $\left.d_{6}\right) \delta \mathrm{ppm} 166.4,160.6,157.9,145$ (q, $\left.J=6.8 \mathrm{~Hz}\right), 134,132.3,132.1,129.9$, 129, $125(q, J=267.6 \mathrm{~Hz}), 124.6,124,116,105.6,98.9,95(q, J=37.8 \mathrm{~Hz}), 56,55.7,37.6$

${ }^{19}$ F NMR $\left(376 \mathrm{MHz}, \mathrm{DMSO}-d_{6}\right) \delta \mathrm{ppm}-51.8$

HRMS (ESI): calcd. for $\mathrm{C}_{19} \mathrm{H}_{17} \mathrm{~F}_{3} \mathrm{~N} \mathrm{O}_{3}\left([\mathrm{M}+\mathrm{H}]^{+}\right)$364.1155, found 364.1157

mp: $132.0-134.8^{\circ} \mathrm{C}$

(3Z)-2-[(2,4-dimethoxyphenyl)methyl]-3-(2,2,2-trifluoroethylidene)isoindolin-1-one 4cZ IR (neat): 1719, 1660, 1610, 1134, $1095 \mathrm{~cm}^{-1}$

${ }^{1}$ H NMR $\left(400 \mathrm{MHz}\right.$, DMSO-d $\left.d_{6}\right) \delta$ ppm $8.22(\mathrm{~d}, J=7.7 \mathrm{~Hz}, 1 \mathrm{H}), 7.87(\mathrm{~d}, J=7.5 \mathrm{~Hz}, 1 \mathrm{H}), 7.81(\mathrm{tt}, J=7.7$, $1.1 \mathrm{~Hz}, 1 \mathrm{H}), 7.73(\mathrm{t}, J=7.5 \mathrm{~Hz}, 1 \mathrm{H}), 6.59(\mathrm{df}, J=2.3 \mathrm{~Hz}, 1 \mathrm{H}), 6.49(\mathrm{~d}, J=8.3 \mathrm{~Hz}, 1 \mathrm{H}), 6.37(\mathrm{dd}, J=8.4$, $2.3 \mathrm{~Hz}, 1 \mathrm{H}), 6.33(\mathrm{q}, J=10.0 \mathrm{~Hz}, 1 \mathrm{H}), 4.97(\mathrm{sl}, 2 \mathrm{H}), 3.83(\mathrm{~s}, 3 \mathrm{H}), 3.71(\mathrm{~s}, 3 \mathrm{H})$

${ }^{13}$ C NMR (75 MHz, DMSO-d $\left.d_{6}\right) \delta$ ppm 168.1, 159.6, 157.1, 141.9 (q, $\left.J=6.4 \mathrm{~Hz}\right),, 137.3,134,132,127$, $125.4,123.7(q, J=267.8 \mathrm{~Hz}), 123.7,121.9,116.5,104.6,98.5,91.8(q, J=37.6 \mathrm{~Hz}), 56,55.6,40.3$

${ }^{19}$ F NMR $\left(376 \mathrm{MHz}\right.$, DMSO- $\left.d_{6}\right) \delta \mathrm{ppm}-49.3$

HRMS (ESI): calcd. for $\mathrm{C}_{19} \mathrm{H}_{17} \mathrm{~F}_{3} \mathrm{~N} \mathrm{O}_{3}\left([\mathrm{M}+\mathrm{H}]^{+}\right)$364.1155, found 364.1157

$\mathrm{mp}: 137.0-139.5^{\circ} \mathrm{C}$ 


\section{(3E/Z)-2-methyl-3-(2,2,2-trifluoroethylidene)isoindolin-1-one 4d}<smiles>CN1C(=O)c2ccccc2/C1=C\C(F)(F)F</smiles>

Starting from N-ethynyl- $\mathrm{N}$-methyl-benzamide $3 \mathrm{~d}(64 \mathrm{mg}, 0.4 \mathrm{mmol})$, Togni I reagent $(139 \mathrm{mg}, 0.42$ $\mathrm{mmol})$ and $\operatorname{Ir}(\mathrm{ppy})_{3}(6.6 \mathrm{mg}, 0.01 \mathrm{mmol})$, using General procedure 3 and stirring at $27^{\circ} \mathrm{C}$ for $4 \mathrm{~h}$. The crude was purified by silica gel flash column chromatography to afford (3E)-2-methyl-3-(2,2,2trifluoroethylidene)isoindolin-1-one $3 \mathrm{dE}$ as a white solid (27 mg) and (3z)-2-methyl-3-(2,2,2trifluoroethylidene)isoindolin-1-one $3 \mathrm{dZ}$ as a white solid ( $16 \mathrm{mg}$ ) (E/Z ratio $63 / 37,47 \%$ overall yield).

\section{(3E)-2-methyl-3-(2,2,2-trifluoroethylidene)isoindolin-1-one 4dE} IR (neat): 1716, 1654, 1336, $1124 \mathrm{~cm}^{-1}$

${ }^{1} \mathrm{H}$ NMR $\left.(500 \mathrm{MHz} \text {, DMSO-d })_{6}\right) \delta \mathrm{ppm} 7.95(\mathrm{~d}, J=7.8 \mathrm{~Hz}, 1 \mathrm{H}), 7.85$ (d, $\left.J=7.5 \mathrm{~Hz}, 1 \mathrm{H}\right), 7.8(\mathrm{td}, J=7.5$, $0.7 \mathrm{~Hz}, 1 \mathrm{H}), 7.72(\mathrm{td}, J=7.5,0.7 \mathrm{~Hz}, 1 \mathrm{H}), 5.99(\mathrm{q}, J=9.4 \mathrm{~Hz}, 1 \mathrm{H}), 3.21(\mathrm{~s}, 3 \mathrm{H})$

${ }^{13} \mathrm{C}$ NMR $\left(125 \mathrm{MHz}\right.$, DMSO- $\left.d_{6}\right) \delta \mathrm{ppm} 165.5,145.1$ (q, $J=6.5 \mathrm{~Hz}$ ), 133.2, 131.6, 131.5, 129.7, 124.3 (q, $J=268.4 \mathrm{~Hz}), 123.9,123.2,94.6(q, J=37.6 \mathrm{~Hz}), 26$

${ }^{19}$ F NMR $\left(376 \mathrm{MHz}\right.$, DMSO- $\left.d_{6}\right) \delta \mathrm{ppm}-51.8$

HRMS (ESI): calcd. for $\mathrm{C}_{11} \mathrm{H}_{9} \mathrm{~F}_{3} \mathrm{NO}\left([\mathrm{M}+\mathrm{H}]^{+}\right)$228.0631, found 228.0632

$\mathrm{mp}: 100.5-105.3^{\circ} \mathrm{C}$

(3Z)-2-methyl-3-(2,2,2-trifluoroethylidene)isoindolin-1-one $4 d Z$ IR (neat): $1721,1655,1121,1090 \mathrm{~cm}^{-1}$

${ }^{1}$ H NMR $\left.(500 \mathrm{MHz}, \text { DMSO-d })_{6}\right) \delta$ ppm $8.14(\mathrm{~d}, J=7.6 \mathrm{~Hz}, 1 \mathrm{H}), 7.82(\mathrm{~d}, J=7.4 \mathrm{~Hz}, 1 \mathrm{H}), 7.76(\mathrm{td}, J=7.6$, $1.1 \mathrm{~Hz}, 1 \mathrm{H}), 7.68(\mathrm{td}, J=7.5,0.6 \mathrm{~Hz}, 1 \mathrm{H}), 6.32(\mathrm{q}, J=7.7 \mathrm{~Hz}, 1 \mathrm{H}), 3.32\left(\mathrm{q}^{6},, J=2.3 \mathrm{~Hz}, 3 \mathrm{H}\right)$

${ }^{13} \mathrm{C}$ NMR $\left(125 \mathrm{MHz}\right.$, DMSO- $\left.d_{6}\right) \delta \mathrm{ppm} 167.9,143.3$ (q, $\left.J=6.3 \mathrm{~Hz}\right), 136.9,133.8,131.5,127.4,123.9$ (q, $J=268.0 \mathrm{~Hz}$ ), 123.2, 121.6, $91.4(q, J=37.3 \mathrm{~Hz}), 28.1$

${ }^{19}$ F NMR $\left(376 \mathrm{MHz}, \mathrm{DMSO}-d_{6}\right) \delta \mathrm{ppm}-47.1$

HRMS (ESI): calcd. for $\mathrm{C}_{11} \mathrm{H}_{9} \mathrm{~F}_{3} \mathrm{NO}\left([\mathrm{M}+\mathrm{H}]^{+}\right)$228.0631, found 228.0632

mp: $105.5-107.0^{\circ} \mathrm{C}$

\footnotetext{
${ }^{6}$ Through space coupling
} 
(3E/Z)-2-benzyl-5-methyl-3-(2,2,2-trifluoroethylidene)isoindolin-1-one 4e<smiles>Cc1ccc2c(c1)/C(=C\C(F)(F)F)N(Cc1ccccc1)C2=O</smiles>

Starting from N-benzyl-N-ethynyl-4-methyl-benzamide $3 e(100 \mathrm{mg}, 0.4 \mathrm{mmol})$, Togni I reagent (158 $\mathrm{mg}, 0.48 \mathrm{mmol})$ and $\operatorname{Ir}(\mathrm{ppy})_{3}(6.6 \mathrm{mg}, 0.01 \mathrm{mmol})$, using General procedure 3 and stirring at $27^{\circ} \mathrm{C}$ for $5.3 \mathrm{~h}$. The crude was purified by silica gel flash column chromatography to afford a mixture of (3E/Z)2-benzyl-5-methyl-3-(2,2,2-trifluoroethylidene)isoindolin-1-one $4 \mathrm{e}$ as a white solid (66 mg, $52 \%$ yield) (E/Z mixture 58/42).

(3E)-2-benzyl-5-methyl-3-(2,2,2-trifluoroethylidene)isoindolin-1-one 4eE IR (neat): 1718, 1654, 1317, 1257, 1157, $1134 \mathrm{~cm}^{-1}$

${ }^{1} \mathrm{H}$ NMR $\left(500 \mathrm{MHz}\right.$, DMSO-d $\left.d_{6}\right) \delta \mathrm{ppm} 7.82(\mathrm{~d}, J=7.7 \mathrm{~Hz}, 1 \mathrm{H}), 7.73(\mathrm{~s}, 1 \mathrm{H}), 7.59(\mathrm{~d}, J=7.7 \mathrm{~Hz}, 1 \mathrm{H})$, 7.38-7.22 (m, $5 \mathrm{H}), 5.88(\mathrm{q}, J=9.5 \mathrm{~Hz}, 1 \mathrm{H}), 5.03(\mathrm{~s}, 2 \mathrm{H}), 2.5(\mathrm{~s}, 3 \mathrm{H}$, under the DMSO peak)

${ }^{13} \mathrm{C}$ NMR $\left(100 \mathrm{MHz}, \mathrm{DMSO}-d_{6}\right) \delta \mathrm{ppm} 166.5,144.6$ (q, $\left.J=6.4 \mathrm{~Hz}\right), 137.7,136.5,133,132.5,129.3(4 \mathrm{C})$, $125.9,127.4,126.8(q, J=268.8 \mathrm{~Hz}), 124.6,123.9,95.7(q, J=37.8 \mathrm{~Hz}), 42.4,22.6$

${ }^{19}$ F NMR $\left(376 \mathrm{MHz}, \mathrm{DMSO}-d_{6}\right) \delta \mathrm{ppm}-51.7$

HRMS (ESI): calcd. for $\mathrm{C}_{18} \mathrm{H}_{15} \mathrm{~F}_{3} \mathrm{~N} \mathrm{O}\left([\mathrm{M}+\mathrm{H}]^{+}\right)$318.1111, found 318.1098

(3Z)-2-benzyl-5-methyl-3-(2,2,2-trifluoroethylidene)isoindolin-1-one 4eZ

${ }^{1}$ H NMR $\left(500 \mathrm{MHz}, \mathrm{DMSO}-d_{6}\right) \delta \mathrm{ppm} 8.06(\mathrm{~s}, 1 \mathrm{H}), 7.78(\mathrm{~d}, J=7.6 \mathrm{~Hz}, 1 \mathrm{H}), 7.55(\mathrm{~d}, J=7.8 \mathrm{~Hz}, 1 \mathrm{H})$, 7.36-7.21 (m, $3 \mathrm{H}), 7.06(\mathrm{~d}, J=7.5 \mathrm{~Hz}, 2 \mathrm{H}), 6.29(\mathrm{q}, J=9.9 \mathrm{~Hz}, 1 \mathrm{H}), 5.09(\mathrm{~s}, 2 \mathrm{H}), 2.5(\mathrm{~s}, 3 \mathrm{H}$, under the DMSO peak)

${ }^{13} \mathrm{C}$ NMR (100 MHz, DMSO-d $\left.d_{6}\right) \delta$ ppm 168.4, $142.1(\mathrm{q}, J=6.5 \mathrm{~Hz}), 137.6,137.2,137.1,132.5,129.0$ (2C), 126.5, 125.2, 124.8 (q, J = 267.9 Hz), 124.7, 123.6, 122.2, 91.4 (q, J = 37.6 Hz), 44.8, 22.5

${ }^{19}$ F NMR $\left(376 \mathrm{MHz}, \mathrm{DMSO}-d_{6}\right) \delta \mathrm{ppm}-48.8$

(3E/Z)-2-benzyl-5-chloro-3-(2,2,2-trifluoroethylidene)isoindolin-1-one 4f<smiles>CCCc1ccccc1CN1C(=O)c2ccc(Cl)cc2/C1=C\C(F)(F)F</smiles>

Starting from N-benzyl-4-chloro-N-ethynyl-benzamide $\mathbf{3 f}(108 \mathrm{mg}, 0.4 \mathrm{mmol})$, Togni I reagent (158 $\mathrm{mg}, 0.48 \mathrm{mmol})$ and $\operatorname{Ir}(\mathrm{ppy})_{3}(6.6 \mathrm{mg}, 0.01 \mathrm{mmol})$, using General procedure 3 and stirring at $27^{\circ} \mathrm{C}$ for $4.7 \mathrm{~h}$. The crude was purified by silica gel flash column chromatography to afford (3E)-2-benzyl-5chloro-3-(2,2,2-trifluoroethylidene)isoindolin-1-one $\mathbf{4 f E}$ as a white solid (50 mg) and (3Z)-2-benzyl-5- 
chloro-3-(2,2,2-trifluoroethylidene)isoindolin-1-one $\mathbf{4 f Z}$ as a white solid (27 mg) (E/Z ratio 63/37, 57\% overall yield).

(3E)-2-benzyl-5-chloro-3-(2,2,2-trifluoroethylidene)isoindolin-1-one 4fE IR (neat): 1309, 1654, 1309, 1269, 1249, $1128 \mathrm{~cm}^{-1}$

${ }^{1} \mathrm{H}$ NMR (400 MHz, DMSO- $\left.d_{6}\right) \delta \mathrm{ppm} 7.97(\mathrm{~d}, J=8.1 \mathrm{~Hz}, 1 \mathrm{H}), 7.86(\mathrm{dd}, J=8.1,1.7 \mathrm{~Hz}, 1 \mathrm{H}), 7.83$ (bs, 1 H), $7.35(\mathrm{t}, J=7.5 \mathrm{~Hz}, 2 \mathrm{H}), 7.31-7.25(\mathrm{~m}, 3 \mathrm{H}), 6.03(\mathrm{q}, J=9.4 \mathrm{~Hz}, 1 \mathrm{H}), 5.05(\mathrm{~s}, 2 \mathrm{H})$

${ }^{13} \mathrm{C}$ NMR (100 MHz, DMSO-d $\left.d_{6}\right) \delta$ ppm 165.4, 143.3 (q, $\left.J=6.4 \mathrm{~Hz}\right), 138.8,136.1,133.7,132.6,129.2$ (2C), 128.8, 128.4, 127.7 (2C), 126, 124.6 (q, J = 269.1 Hz), 124.3, 96.9 (q, J = 37.5 Hz), 42.7

${ }^{19}$ F NMR $\left(376 \mathrm{MHz}\right.$, DMSO- $\left.d_{6}\right) \delta \mathrm{ppm}-51.8$

HRMS (ESI): calcd. for $\mathrm{C}_{17} \mathrm{H}_{12} \mathrm{Cl} \mathrm{F}_{3} \mathrm{NO}\left([\mathrm{M}+\mathrm{H}]^{+}\right)$338.0554, found 338.0556

$\mathrm{mp}: 102.6-110.3^{\circ} \mathrm{C}$

(3Z)-2-benzyl-5-chloro-3-(2,2,2-trifluoroethylidene)isoindolin-1-one 4fZ IR (neat): 1722, 1660, 1301, 1274, 1255, $1134 \mathrm{~cm}^{-1}$

${ }^{1} \mathrm{H}$ NMR $\left(400 \mathrm{MHz}, \mathrm{DMSO}-d_{6}\right) \delta \mathrm{ppm} 8.45(\mathrm{df}, J=1.5 \mathrm{~Hz}, 1 \mathrm{H}), 7.89(\mathrm{~d}, J=8.1 \mathrm{~Hz}, 1 \mathrm{H}), 7.78(\mathrm{dd}, J=$ $8.1,1.6 \mathrm{~Hz}, 1 \mathrm{H}), 7.3(\mathrm{t}, J=7.4 \mathrm{~Hz}, 2 \mathrm{H}), 7.23(\mathrm{t}, J=7.3 \mathrm{~Hz}, 1 \mathrm{H}), 7.07(\mathrm{t}, J=7.5 \mathrm{~Hz}, 2 \mathrm{H}), 6.48(\mathrm{q}, J=9.9$ $\mathrm{Hz}, 1 \mathrm{H}), 5.1(\mathrm{~s}, 2 \mathrm{H})$

${ }^{13} \mathrm{C}$ NMR $\left(100 \mathrm{MHz}\right.$, DMSO- $\left.d_{6}\right) \delta \mathrm{ppm} 168.2,141.3(\mathrm{q}, J=6.4 \mathrm{~Hz}), 139.8,139.3,137.6,132.2,128.9$ (2C), 127.4, 126.1, $125.8(2 \mathrm{C}), 125.5,123.6$ (q, J = 268.8 Hz), 122.4, $93.4(q, J=37.5 \mathrm{~Hz}), 44.9$

${ }^{19}$ F NMR $\left(376 \mathrm{MHz}, \mathrm{DMSO}-d_{6}\right) \delta \mathrm{ppm}-49.1$

HRMS (ESI): calcd. for $\mathrm{C}_{17} \mathrm{H}_{12} \mathrm{Cl} \mathrm{F}_{3} \mathrm{~N} \mathrm{O}\left([\mathrm{M}+\mathrm{H}]^{+}\right)$338.0554, found 338.0556

$\mathrm{mp}: 138.2-146.4^{\circ} \mathrm{C}$

\section{(3E/Z)-2-benzyl-5-fluoro-3-(2,2,2-trifluoroethylidene)isoindolin-1-one 4g}<smiles>O=C1c2ccc(F)cc2/C(=C\C(F)(F)F)N1Cc1ccccc1</smiles>

Starting from N-benzyl-N-ethynyl-4-fluoro-benzamide $3 \mathrm{~g}(101 \mathrm{mg}, 0.4 \mathrm{mmol})$, Togni I reagent (158 $\mathrm{mg}, 0.48 \mathrm{mmol}$ ) and $\operatorname{Ir}(\mathrm{ppy})_{3}(6.6 \mathrm{mg}, 0.01 \mathrm{mmol})$, using General procedure 3 (in $\mathrm{CH}_{2} \mathrm{Cl}_{2}$ ) and stirring at $27^{\circ} \mathrm{C}$ for $6.5 \mathrm{~h}$. The crude was purified by silica gel flash column chromatography to afford a mixture of (3E/Z)-2-benzyl-5-fluoro-3-(2,2,2-trifluoroethylidene)isoindolin-1-one $\mathbf{4 g}$ as a yellow oil (74 $\mathrm{mg}, 58 \%$ yield) (E/Z mixture 62/38).

(3E)-2-benzyl-5-fluoro-3-(2,2,2-trifluoroethylidene)isoindolin-1-one 4gE IR (neat): 1730, 1658, $1109 \mathrm{~cm}^{-1}$ 
${ }^{1} \mathrm{H}$ NMR $\left(500 \mathrm{MHz}, \mathrm{DMSO}-\mathrm{d}_{6}\right) \delta \mathrm{ppm} 8.01(\mathrm{dd}, J=8.4,5.2 \mathrm{~Hz}, 1 \mathrm{H}), 7.65(\mathrm{td}, J=8.6 \mathrm{~Hz}, 1 \mathrm{H}), 7.6(\mathrm{~d}, J=$ $8.7 \mathrm{~Hz}, 1 \mathrm{H}), 7.35(\mathrm{t}, J=7.4 \mathrm{~Hz}, 2 \mathrm{H}), 7.28(\mathrm{~m}, 3 \mathrm{H}), 6.02(\mathrm{q}, J=9.4 \mathrm{~Hz}, 1 \mathrm{H}), 5.05(\mathrm{~s}, 2 \mathrm{H})$

${ }^{13}$ C NMR $\left(125 \mathrm{MHz}\right.$, DMSO- $\left.d_{6}\right) \delta \mathrm{ppm} 165.5,165.5(\mathrm{~d}, J=250.1 \mathrm{~Hz}), 143.4(q, J=6.3 \mathrm{~Hz}), 136.5,134.3$ (2C), 129.1 (2C), $127.7(2 \mathrm{C}), 126.4,126.2,124.4$ (q, $J=268.0 \mathrm{~Hz}$ ), 119.9 (d, $J=23.5 \mathrm{~Hz}, 2 \mathrm{C}$ ), 112.1, 96.5 $(q, J=37.7 \mathrm{~Hz}), 42.7$

${ }^{19}$ F NMR $\left(376 \mathrm{MHz}\right.$, DMSO- $\left.d_{6}\right) \delta \mathrm{ppm}-51.9,-105$

HRMS (EI) : calcd. for $\mathrm{C}_{17} \mathrm{H}_{11} \mathrm{~F}_{4} \mathrm{~N} O\left([\mathrm{M}]^{+}\right)$321.0777, found 321.0772

(3Z)-2-benzyl-5-fluoro-3-(2,2,2-trifluoroethylidene)isoindolin-1-one $4 \mathrm{gz}$

${ }^{1} \mathrm{H}$ NMR $\left(400 \mathrm{MHz}, \mathrm{DMSO}-d_{6}\right) \delta \mathrm{ppm} 8.2(\mathrm{dd}, J=8.8,2.2 \mathrm{~Hz}, 1 \mathrm{H}), 7.94(\mathrm{dd}, J=8.5 \mathrm{~Hz}, 1 \mathrm{H}), 7.57(\mathrm{td}, J$ $=8.6,2.3 \mathrm{~Hz}, 1 \mathrm{H}), 7.3(\mathrm{t}, J=7.6 \mathrm{~Hz}, 2 \mathrm{H}), 7.23(\mathrm{t}, J=7.4 \mathrm{~Hz}, 1 \mathrm{H}), 7.07(\mathrm{~d}, J=7.5 \mathrm{~Hz}, 2 \mathrm{H}), 6.42(\mathrm{q}, J=$ $10.0 \mathrm{~Hz}, 1 \mathrm{H}), 5.1(\mathrm{~s}, 2 \mathrm{H})$

${ }^{13}$ C NMR (100 MHz, DMSO- $\left.d_{6}\right) \delta$ ppm 166.8, $165.5(d, J=249.9 \mathrm{~Hz}), 140.7(q, J=6.4 \mathrm{~Hz}), 139.9(\mathrm{~d}, J=$ $11.1 \mathrm{~Hz}, 136.4,128.7(2 \mathrm{C}), 127.1,126.5(\mathrm{~d}, J=10.1 \mathrm{~Hz}), 123.8(2 \mathrm{C}), 123.8(\mathrm{~d},, 123.6$ (q, J = 268.3 Hz), $119.6(d, J=24.1 \mathrm{~Hz}), 109.5(\mathrm{~d}, J=26.3 \mathrm{~Hz}), 93.1(\mathrm{q}, J=37.8 \mathrm{~Hz}), 44.9$

${ }^{19}$ F NMR (376 MHz, dmso-d6) $\delta$ ppm -49.1,-105

HRMS (ESI): calcd. for $\mathrm{C}_{17} \mathrm{H}_{12} \mathrm{~F}_{4} \mathrm{NO}\left([\mathrm{M}+\mathrm{H}]^{+}\right) 322.0850$, found 322.0852

\section{(3E/Z)-2-benzyl-3-(2,2,2-trifluoroethylidene)-5-(trifluoromethyl)isoindolin-1-one $4 \mathrm{~h}$}<smiles></smiles>

Starting from N-benzyl-N-ethynyl-4-(trifluoromethyl)benzamide $3 \mathrm{~h}$ (121 mg, $0.4 \mathrm{mmol}$ ), Togni I reagent $(158 \mathrm{mg}, 0.48 \mathrm{mmol})$ and $\operatorname{Ir}(\mathrm{ppy})_{3}(6.6 \mathrm{mg}, 0.01 \mathrm{mmol})$, using General procedure 3 and stirring at $27^{\circ} \mathrm{C}$ for $4.7 \mathrm{~h}$. The crude was purified by silica gel flash column chromatography to afford (3E)-2-benzyl-3-(2,2,2-trifluoroethylidene)-5-(trifluoromethyl)isoindolin-1-one $\mathbf{4 h E}$ as a colorless oil (65 mg) and (3Z)-2-benzyl-3-(2,2,2-trifluoroethylidene)-5-(trifluoromethyl)isoindolin-1-one $\mathbf{4 h Z}$ as a yellow oil ( $8 \mathrm{mg})$ (E/Z mixture $90 / 10,45 \%$ overall yield).

(3E)-2-benzyl-3-(2,2,2-trifluoroethylidene)-5-(trifluoromethyl)isoindolin-1-one $4 \mathrm{hE}$ IR (neat): 1733, 1313, 1120, $1103 \mathrm{~cm}^{-1}$

${ }^{1}$ H NMR (500 MHz, DMSO-d 6 ) $\delta$ ppm $8.17(\mathrm{~s}, 2 \mathrm{H}), 8.11(\mathrm{sl}, 1 \mathrm{H}), 7.35(\mathrm{t}, J=7.4 \mathrm{~Hz}, 2 \mathrm{H}), 7.3(\mathrm{~m}, 3 \mathrm{H})$, $6.13(q, J=9.3 \mathrm{~Hz}, 1 \mathrm{H}), 5.1(\mathrm{~s}, 2 \mathrm{H})$

${ }^{13} \mathrm{C}$ NMR $\left(125 \mathrm{MHz}\right.$, DMSO- $\left.d_{6}\right) \delta \mathrm{ppm} 165.5,143.3(\mathrm{q}, J=6.2 \mathrm{~Hz}), 136.2,133.7,133.5,132.6,129.7$ $(\mathrm{bq}, J=3.3 \mathrm{~Hz}$ ), $129(2 \mathrm{C}), 127.6(2 \mathrm{C}), 127.6,125,124.5(\mathrm{q}, J=268.6 \mathrm{~Hz}), 124$ (q, $J=272.7 \mathrm{~Hz}), 120.7$ $(\mathrm{bq}, J=5.0 \mathrm{~Hz}), 97.4(\mathrm{q}, J=37.6 \mathrm{~Hz}), 42.9$

${ }^{19}$ F NMR $\left(376 \mathrm{MHz}\right.$, DMSO- $\left.d_{6}\right) \delta \mathrm{ppm}-51.9,-61.6$ 
HRMS (EI) : calcd. for $\mathrm{C}_{18} \mathrm{H}_{11} \mathrm{~F}_{6} \mathrm{~N} O\left([\mathrm{M}]^{+}\right)$371.0739, found 371.0730

(3Z)-2-benzyl-3-(2,2,2-trifluoroethylidene)-5-(trifluoromethyl)isoindolin-1-one $4 \mathrm{hZ}$ IR (neat): 1724, 1660, 1323, 1130, 1110, $1097 \mathrm{~cm}^{-1}$

${ }^{1} \mathrm{H}$ NMR $\left(500 \mathrm{MHz}\right.$, DMSO- $\left.d_{6}\right) \delta \mathrm{ppm} 8.78(\mathrm{~s}, 1 \mathrm{H}), 8.08(\mathrm{bs}, 2 \mathrm{H}), 7.3(\mathrm{t}, J=7.5 \mathrm{~Hz}, 2 \mathrm{H}), 7.24(\mathrm{t}, J=7.3$ $\mathrm{Hz}, 1 \mathrm{H}), 7.1(\mathrm{~d}, J=765 \mathrm{~Hz}, 2 \mathrm{H}), 6.69(\mathrm{q}, J=10.09 \mathrm{~Hz}, 1 \mathrm{H}), 5.14(\mathrm{~s}, 2 \mathrm{H})$

${ }^{13} \mathrm{C}$ NMR $\left(100 \mathrm{MHz}, \mathrm{DMSO}-d_{6}\right) \delta \mathrm{ppm} 167.1,141(\mathrm{q}, J=6.1 \mathrm{~Hz}), 138,136.7,133.9(\mathrm{q}, J=32.0 \mathrm{~Hz})$, $130.8,128.8(\mathrm{bq}, J=3.7 \mathrm{~Hz}), 128.6(2 \mathrm{C}), 127,126(2 \mathrm{C}), 124.5,124.2(\mathrm{q}, J=272.8 \mathrm{~Hz}), 123.6(\mathrm{q}, J=$ $268.5 \mathrm{~Hz}), 119.4(\mathrm{bq}, J=3.8 \mathrm{~Hz}), 93.8(\mathrm{q}, J=37.6 \mathrm{~Hz}), 45$

${ }^{19}$ F NMR $\left(376 \mathrm{MHz}, \mathrm{DMSO}-d_{6}\right) \delta \mathrm{ppm}-49.2,-61$

HRMS (EI): calcd. for $\mathrm{C}_{18} \mathrm{H}_{11} \mathrm{~F}_{6} \mathrm{NO}\left([\mathrm{M}]^{+}\right)$371.0739, found 371.0737

\section{(3E/Z)-2-benzyl-5-tert-butyl-3-(2,2,2-trifluoroethylidene)isoindolin-1-one 4i}<smiles>CC(C)(C)c1ccc2c(c1)C(=CC(F)(F)F)N(Cc1ccccc1)C2=O</smiles>

Starting from N-benzyl-4-tert-butyl-N-ethynyl-benzamide $3 \mathbf{i}(77 \mathrm{mg}, 0.26 \mathrm{mmol}$ ), Togni I reagent (105 $\mathrm{mg}, 0.32 \mathrm{mmol})$ and $\operatorname{Ir}(\mathrm{ppy})_{3}(4.3 \mathrm{mg}, 0.007 \mathrm{mmol})$, using General procedure 3 and stirring at $27^{\circ} \mathrm{C}$ for $3.8 \mathrm{~h}$. The crude was purified by silica gel flash column chromatography to afford (3E)-2-benzyl-5tert-butyl-3-(2,2,2-trifluoroethylidene)isoindolin-1-one $4 \mathrm{iE}$ as a pale yellow oil (25 $\mathrm{mg}$ ) and (3Z)-2benzyl-5-tert-butyl-3-(2,2,2-trifluoroethylidene)isoindolin-1-one $4 \mathrm{iZ}$ as a pale yellow oil $(18 \mathrm{mg})(\mathrm{E} / \mathrm{Z}$ mixture $56 / 44,40 \%$ overall yield).

\section{(3E)-2-benzyl-5-tert-butyl-3-(2,2,2-trifluoroethylidene)isoindolin-1-one 4iE}

IR (neat): 1726, 1654, 1313, 1253, $1126 \mathrm{~cm}^{-1}$

${ }^{1}$ H NMR $\left(400 \mathrm{MHz}, \mathrm{DMSO}-d_{6}\right) \delta \mathrm{ppm} 8(\mathrm{sb}, 1 \mathrm{H}), 7.85(\mathrm{~d}, J=8.0 \mathrm{~Hz}, 1 \mathrm{H}), 7.82(\mathrm{~d}, J=8.0,1.5 \mathrm{~Hz}, 1 \mathrm{H})$, 7.4-7.2 (m, $5 \mathrm{H}), 5.9(\mathrm{q}, J=9.6 \mathrm{~Hz}, 1 \mathrm{H}), 5.03(\mathrm{~s}, 2 \mathrm{H}), 1.32(\mathrm{~s}, 9 \mathrm{H})$

${ }^{13}$ C NMR $\left(100 \mathrm{MHz}\right.$, DMSO- $\left.d_{6}\right) \delta \mathrm{ppm} 166.5,157.2,144.7$ (q, $\left.J=6.1 \mathrm{~Hz}\right), 136.6,132.4,129.8,129.2$ (2C), 127.9, 127.4, $127.3(2 \mathrm{C}), 123.9$ (q, $J=268.3 \mathrm{~Hz}$ ), 123.5, 121.3, 95.4 (q, J = 37.8 Hz), 42.5, 35.7, $31.2(3 \mathrm{C})$

${ }^{19}$ F NMR $\left(376 \mathrm{MHz}, \mathrm{DMSO}-d_{6}\right) \delta \mathrm{ppm}-51.9$

HRMS (ESI): calcd. for $\mathrm{C}_{21} \mathrm{H}_{22} \mathrm{~F}_{3} \mathrm{~N} \mathrm{O}\left([\mathrm{M}+\mathrm{H}]^{+}\right) 360.1570$, found 360.157 
(3Z)-2-benzyl-5-tert-butyl-3-(2,2,2-trifluoroethylidene)isoindolin-1-one 4iz IR (neat): 1728, 1660, 1267, 1251, 1197, $1128 \mathrm{~cm}^{-1}$

${ }^{1}$ H NMR (400 MHz, DMSO-d $\left.d_{6}\right) \delta$ ppm $8.3(\mathrm{sb}, 1 \mathrm{H}), 7.8(\mathrm{db}, J=7.9 \mathrm{~Hz}, 1 \mathrm{H}$ ), $7.76(\mathrm{dd}, J=8.0,1.4 \mathrm{~Hz}, 1$ H), $7.3(\mathrm{t}, J=7.7 \mathrm{~Hz}, 2 \mathrm{H}), 7.23(\mathrm{t}, J=7.4 \mathrm{~Hz}, 1 \mathrm{H}), 7.05(\mathrm{~d}, J=7.6 \mathrm{~Hz}, 2 \mathrm{H}), 6.5(\mathrm{q}, J=10.1 \mathrm{~Hz}, 1 \mathrm{H}), 5.1$ $(\mathrm{s}, 2 \mathrm{H}), 1.4(\mathrm{~s}, 9 \mathrm{H})$

${ }^{13}$ C NMR (100 MHz, DMSO-d $\left.d_{6}\right) \delta$ ppm 169, 158, 142.2 (q, $\left.J=6.5 \mathrm{~Hz}\right), 137.5,137.2,129,128.9(2 \mathrm{C})$, $127.3,125.8(2 \mathrm{C}), 124.8,124.0(\mathrm{q}, J=268.3 \mathrm{~Hz}), 123.6,119.3,91.5(\mathrm{q}, J=37.4 \mathrm{~Hz}), 44.5,35.9,31.5$ (3C)

${ }^{19}$ F NMR $\left(376 \mathrm{MHz}, \mathrm{DMSO}-d_{6}\right) \delta \mathrm{ppm}-48.7$

HRMS (ESI): calcd. for $\mathrm{C}_{21} \mathrm{H}_{21} \mathrm{~F}_{3} \mathrm{~N} \mathrm{O}\left([\mathrm{M}+\mathrm{H}]^{+}\right) 360.1570$, found 360.157

\section{(3E/Z)-2-benzyl-5-nitro-3-(2,2,2-trifluoroethylidene)isoindolin-1-one 4j}<smiles>O=C1c2ccc([N+](=O)[O-])cc2/C(=C\C(F)(F)F)N1Cc1ccccc1</smiles>

Starting from N-benzyl- $\mathrm{N}$-ethynyl-4-nitro-benzamide $3 \mathbf{j}(112 \mathrm{mg}, 0.4 \mathrm{mmol})$, Togni I reagent $(158 \mathrm{mg}$, $0.48 \mathrm{mmol})$ and $\operatorname{Ir}(\mathrm{ppy})_{3}(6.6 \mathrm{mg}, 0.01 \mathrm{mmol})$, using General procedure 3 and stirring at $27^{\circ} \mathrm{C}$ for $22 \mathrm{~h}$ and then at $40^{\circ} \mathrm{C}$ for $5.5 \mathrm{~h}$. The crude was purified by silica gel flash column chromatography to afford a mixture of (3E/Z)-2-benzyl-5-nitro-3-(2,2,2-trifluoroethylidene)isoindolin-1-one $\mathbf{4} \mathbf{j}$ as a white solid (80 mg, $52 \%$ yield) (E/Z mixture 55/45).

(3E)-2-benzyl-5-nitro-3-(2,2,2-trifluoroethylidene)isoindolin-1-one 4jE IR (neat): 1730, 1656, 1533, 1340, 1116, 779, $738 \mathrm{~cm}^{-1}$ (mixture 70/30 E/Z)

${ }^{1} \mathrm{H}$ NMR (400 MHz, DMSO-d 6 ) $\delta$ ppm 8.63 (df, $J=1.5 \mathrm{~Hz}, 1 \mathrm{H}$ ), 8.59 (dd, $\left.J=8.3,1.8 \mathrm{~Hz}, 1 \mathrm{H}\right), 8.19$ (d, $J$ $=8.3 \mathrm{~Hz}, 1 \mathrm{H}), 7.39-7.3(\mathrm{~m}, 5 \mathrm{H}), 6.18(\mathrm{q}, J=9.3 \mathrm{~Hz}, 1 \mathrm{H}), 5.1(\mathrm{~s}, 2 \mathrm{H})$

${ }^{13} \mathrm{C}$ NMR $\left(100 \mathrm{MHz}\right.$, DMSO- $\left.d_{6}\right) \delta \mathrm{ppm} 164.5,151.1,143(\mathrm{q}, J=6.0 \mathrm{~Hz}), 134.6,132.8,128.9,128.7(2 \mathrm{C})$, $127.6(2 \mathrm{C}), 127.6,125.9,125.9,124.4(\mathrm{q}, J=268.9 \mathrm{~Hz}), 119.9,97.6$ (q, $J=37.8 \mathrm{~Hz}), 43.5$

${ }^{19}$ F NMR $\left(376 \mathrm{MHz}\right.$, DMSO- $\left.d_{6}\right) \delta \mathrm{ppm}-51.6$

HRMS (ESI): calcd. for $\mathrm{C}_{17} \mathrm{H}_{11} \mathrm{~F}_{3} \mathrm{~N}_{2} \mathrm{O}\left([\mathrm{M}+\mathrm{H}]^{+}\right)$3498.0795, found 349.0796

(3Z)-2-benzyl-5-nitro-3-(2,2,2-trifluoroethylidene)isoindolin-1-one 4jZ

${ }^{1} \mathrm{H}$ NMR $\left(400 \mathrm{MHz}\right.$, DMSO- $\left.d_{6}\right) \delta \mathrm{ppm} 9.21(\mathrm{df}, J=1.8 \mathrm{~Hz}, 1 \mathrm{H}), 8.52(\mathrm{dd}, J=8.26,1.9 \mathrm{~Hz}, 1 \mathrm{H}), 8.13(\mathrm{~d}, J$ $=8.3 \mathrm{~Hz}, 1 \mathrm{H}), 7.31(\mathrm{~m}, 2 \mathrm{H}), 7.23(\mathrm{t}, J=7.3 \mathrm{~Hz}, 1 \mathrm{H}), 7.11(\mathrm{~d}, J=7.4 \mathrm{~Hz}, 2 \mathrm{H}), 6.81(\mathrm{q}, J=10.0 \mathrm{~Hz}, 1 \mathrm{H})$, $5.14(\mathrm{~s}, 2 \mathrm{H})$

${ }^{13} \mathrm{C}$ NMR $\left(100 \mathrm{MHz}\right.$, DMSO- $\left.d_{6}\right) \delta \mathrm{ppm} 167.1,151.6,140.6(\mathrm{q}, J=6.5 \mathrm{~Hz}), 138.5,135.9,132.2,128.0$ (2C), 127.6, 127.2, 125.9 (2C), 125.3, 124.4 (q, J = 267.2 Hz), 117, 95 (q, $J=37.6 \mathrm{~Hz}$ ), 45.6

${ }^{19}$ F NMR $\left(376 \mathrm{MHz}, \mathrm{DMSO}-d_{6}\right) \delta \mathrm{ppm}-49.3$

HRMS (ESI): calcd. for $\mathrm{C}_{17} \mathrm{H}_{11} \mathrm{~F}_{3} \mathrm{~N}_{2} \mathrm{O}\left([\mathrm{M}+\mathrm{H}]^{+}\right)$3498.0795, found 349.0796 


\section{(3E/Z)-2-benzyl-1-oxo-3-(2,2,2-trifluoroethylidene)isoindoline-5-carbonitrile 4k}<smiles>N#Cc1ccc2c(c1)C(=CC(F)(F)F)N(Cc1ccccc1)C2=O</smiles>

Starting from N-benzyl-4-cyano-N-ethynyl-benzamide 3k (104 mg, $0.4 \mathrm{mmol})$, Togni I reagent (158 $\mathrm{mg}, 0.48 \mathrm{mmol})$ and $\operatorname{Ir}(\mathrm{ppy})_{3}(6.6 \mathrm{mg}, 0.01 \mathrm{mmol})$, using General procedure 3 and stirring at $27^{\circ} \mathrm{C}$ for $6 \mathrm{~h}$ and at $40^{\circ} \mathrm{C}$ for $15 \mathrm{~h}$. The crude was purified by silica gel flash column chromatography to afford a mixture of (3E)-2-benzyl-1-oxo-3-(2,2,2-trifluoroethylidene)isoindoline-5-carbonitrile $4 \mathbf{k}$ as a white solid (64 mg) (E/Z mixture 66/34, $56 \%$ overall yield).

(3E)-2-benzyl-1-oxo-3-(2,2,2-trifluoroethylidene)isoindoline-5-carbonitrile 4kE IR (neat): 2233, 1735, 1654, 1107, 704, $661 \mathrm{~cm}^{-1}$

${ }^{1} \mathrm{H}$ NMR $\left(500 \mathrm{MHz}, \mathrm{DMSO}-d_{6}\right) \delta \mathrm{ppm} 8.27(\mathrm{~d}, J=7.8 \mathrm{~Hz}, 1 \mathrm{H}), 8.16(\mathrm{bs}, 1 \mathrm{H}), 8.13(\mathrm{~d}, J=7.8 \mathrm{~Hz}, 1 \mathrm{H})$, $7.35(\mathrm{t}, J=7.4 \mathrm{~Hz}, 2 \mathrm{H}), 7.28(\mathrm{~m}, 3 \mathrm{H}), 6.13(\mathrm{q}, J=9.3 \mathrm{~Hz}, 1 \mathrm{H}), 5.09(\mathrm{~s}, 2 \mathrm{H})$

${ }^{13} \mathrm{C}$ NMR $\left(125 \mathrm{MHz}\right.$, DMSO- $\left.d_{6}\right) \delta$ ppm 165.1, $142.9(\mathrm{q}, J=6.2 \mathrm{~Hz}), 136.4,136.1,133.9,132.6,129.1$ (2C), 128.1, $127.4(2 \mathrm{C}), 125.4,125.3,124.4$ (q, J = 269.7 Hz), 118.5, 116.3, 97.6 (q, J = 37.7 Hz), 43

${ }^{19}$ F NMR (376 MHz, DMSO- $\left.d_{6}\right) \delta \mathrm{ppm}-51.8$

HRMS (EI): calcd. for $\mathrm{C}_{18} \mathrm{H}_{11} \mathrm{~F}_{3} \mathrm{~N}_{2} \mathrm{O}\left([\mathrm{M}]^{+}\right) 328.0823$, found 328.0818

mp: $122.6-132.0^{\circ} \mathrm{C}$ (mixture $65 / 35 \mathrm{E} / \mathrm{Z}$ )

(3Z)-2-benzyl-1-oxo-3-(2,2,2-trifluoroethylidene)isoindoline-5-carbonitrile 4kZ IR (neat): 2231, 1727, 1662, $1094 \mathrm{~cm}^{-1}$

${ }^{1} \mathrm{H}$ NMR $\left(400 \mathrm{MHz}\right.$, DMSO- $\left.d_{6}\right) \delta \mathrm{ppm} 8.84(\mathrm{bs}, 1 \mathrm{H}), 8.18$ (dd, $\left.J=7.9,1.2 \mathrm{~Hz}, 1 \mathrm{H}\right), 8.07(\mathrm{~d}, J=7.8 \mathrm{~Hz}, 1$ H), $7.31(\mathrm{t}, J=7.4 \mathrm{~Hz}, 2 \mathrm{H}), 7.23(\mathrm{t}, J=7.3 \mathrm{~Hz}, 1 \mathrm{H}), 7.1(\mathrm{~d}, J=7.5 \mathrm{~Hz}, 2 \mathrm{H}), 6.53(\mathrm{q}, J=9.9 \mathrm{~Hz}, 1 \mathrm{H}), 5.12$ $(\mathrm{s}, 2 \mathrm{H})$

${ }^{13} \mathrm{C}$ NMR $\left(125 \mathrm{MHz}\right.$, DMSO- $\left.d_{6}\right) \delta \mathrm{ppm} 167,140.8$ (q, J = 6.5 Hz), 137.6, 136.6, 135.5, 130.6, $128.7(2 \mathrm{C})$, $127.3,126.5,125.9(2 \mathrm{C}), 124.9,123.5$ (q, $J=268.9 \mathrm{~Hz}), 118.3,116.2,94.1$ (q, J = 37.6 Hz), 43.8

${ }^{19}$ F NMR $\left(376 \mathrm{MHz}, \mathrm{DMSO}-d_{6}\right) \delta \mathrm{ppm}-49.2$

methyl carboxylate 4I

(3E/Z)-2-benzyl-1-oxo-3-(2,2,2-trifluoroethylidene)isoindoline-5-<smiles>COC(=O)c1ccc2c(c1)/C(=C\C(F)(F)F)N(Cc1ccccc1)C2=O</smiles>

Starting from methyl 4-[benzyl(ethynyl)carbamoyl]benzoate 31 (107 mg, $0.36 \mathrm{mmol})$, Togni I reagent $(145 \mathrm{mg}, 0.44 \mathrm{mmol})$ and $\operatorname{Ir}(\mathrm{ppy})_{3}(6 \mathrm{mg}, 0.09 \mathrm{mmol})$, using General procedure 3 and stirring at $27^{\circ} \mathrm{C}$ 
for $22 \mathrm{~h}$. The crude was purified by silica gel flash column chromatography to afford a mixture of methyl (3E/Z)-2-benzyl-1-oxo-3-(2,2,2-trifluoroethylidene)isoindoline-5-carboxylate $4 \mathrm{I}$ as a yellow solid (81 mg, $61 \%$ yield) (E/Z mixture 63/37).

methyl (3E)-2-benzyl-1-oxo-3-(2,2,2-trifluoroethylidene)isoindoline-5-carboxylate 4IE

${ }^{1} \mathrm{H}$ NMR $\left(500 \mathrm{MHz}, \mathrm{DMSO}-d_{6}\right) \delta \mathrm{ppm} 8.52(\mathrm{~s}, 1 \mathrm{H}), 8.31(\mathrm{dd}, J=7.9,1.2 \mathrm{~Hz}, 1 \mathrm{H}), 8.07(\mathrm{~d}, J=7.9 \mathrm{~Hz}, 1$ H), $7.36(\mathrm{t}, J=7.4 \mathrm{~Hz}, 2 \mathrm{H}), 7.3(\mathrm{~m}, 3 \mathrm{H}), 6.08(\mathrm{q}, J=9.4 \mathrm{~Hz}, 1 \mathrm{H}), 5.08(\mathrm{~s}, 2 \mathrm{H}), 3.94(\mathrm{~s}, 3 \mathrm{H})$

${ }^{13} \mathrm{C}$ NMR $\left(100 \mathrm{MHz}\right.$, DMSO- $\left.d_{6}\right) \delta \mathrm{ppm} 165.6,165.6,143.8$ (q, $\left.J=6.2 \mathrm{~Hz}\right), 136.3,134.2,133.5,132.8$, $132.2,129.2(2 \mathrm{C}), 128(2 \mathrm{C}), 128,125.1,124.9$ (q, $J=268.0 \mathrm{~Hz}), 124.9,96.6(q, J=37.4 \mathrm{~Hz}), 52.8,42.6$

${ }^{19}$ F NMR $\left(376 \mathrm{MHz}, \mathrm{DMSO}-d_{6}\right) \delta \mathrm{ppm}-51.7$

HRMS (ESI): calcd. for $\mathrm{C}_{19} \mathrm{H}_{15} \mathrm{~F}_{3} \mathrm{~N} \mathrm{O}_{3}\left([\mathrm{M}+\mathrm{H}]^{+}\right)$362.0999, found 362.1001

methyl (3Z)-2-benzyl-1-oxo-3-(2,2,2-trifluoroethylidene)isoindoline-5-carboxylate 4IZ

${ }^{1} \mathrm{H}$ NMR $\left(500 \mathrm{MHz}, \mathrm{DMSO}-d_{6}\right) \delta \mathrm{ppm} 8.82(\mathrm{~s}, 1 \mathrm{H}), 8.26(\mathrm{dd}, J=7.8,1.2 \mathrm{~Hz}, 1 \mathrm{H}), 8(\mathrm{~d}, J=7.9 \mathrm{~Hz}, 1 \mathrm{H})$, $7.3(\mathrm{~m}, 2 \mathrm{H}), 7.23(\mathrm{t}, J=7.3 \mathrm{~Hz}, 1 \mathrm{H}), 7.1(\mathrm{~d}, J=7.6 \mathrm{~Hz}, 2 \mathrm{H}), 6.67(\mathrm{q}, J=10.01 \mathrm{~Hz}, 1 \mathrm{H}), 5.13(\mathrm{~s}, 2 \mathrm{H})$, $3.95(\mathrm{~s}, 3 \mathrm{H})$

${ }^{13} \mathrm{C}$ NMR $\left(100 \mathrm{MHz}, \mathrm{DMSO}-d_{6}\right) \delta \mathrm{ppm} 167.6,166,141.1(\mathrm{q}, J=6.4 \mathrm{~Hz}), 137.6,136.8,134.7,132.5$, 130.7, $129.1(2 \mathrm{C}), 127.2,125.5(2 \mathrm{C}), 124.8,124.0(\mathrm{q}, J=268.3 \mathrm{~Hz}), 122.9,94(q, J=37.4 \mathrm{~Hz}$ ), 53.8, 44.7

${ }^{19}$ F NMR $\left(376 \mathrm{MHz}, \mathrm{DMSO}-d_{6}\right) \delta-49.1 \mathrm{ppm}$

\section{(3E/Z)-2-benzyl-3-(2,2,2-trifluoroethylidene)pyrrolo[3,4-c]pyridin-1-one 4m}

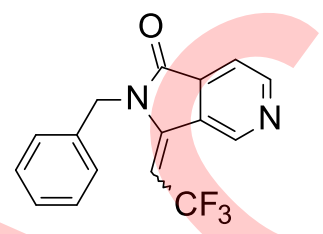

Starting from N-benzyl-N-ethynyl-pyridine-4-carboxamide $3 \mathrm{~m} \mathrm{(95} \mathrm{mg,} 0.4 \mathrm{mmol}$ ), Togni I reagent $(158 \mathrm{mg}, 0.48 \mathrm{mmol})$ and $\operatorname{Ir}(\mathrm{ppy})_{3}(6.6 \mathrm{mg}, 0.01 \mathrm{mmol})$, using General procedure 3 and stirring at $27^{\circ} \mathrm{C}$ for $20 \mathrm{~h}$. The crude was purified by silica gel flash column chromatography to afford (3E)-2benzyl-3-(2,2,2-trifluoroethylidene)pyrrolo[3,4-c]pyridin-1-one $4 \mathrm{mE}$ as a yellow solid (40 $\mathrm{mg}$ ) and (3Z)-2-benzyl-3-(2,2,2-trifluoroethylidene)pyrrolo[3,4-c]pyridin-1-one $4 \mathrm{mz}$ as a yellow oil $(28 \mathrm{mg})$ (E/Z mixture $73 / 27,54 \%$ overall yield).

(3E)-2-benzyl-3-(2,2,2-trifluoroethylidene)pyrrolo[3,4-c]pyridin-1-one $4 \mathrm{mE}$ IR (neat): 1728, 1660, 1334, $1105 \mathrm{~cm}^{-1}$

${ }^{1} \mathrm{H}$ NMR (400 MHz, DMSO- $d_{6}$ ) $\delta$ ppm 9.15 (bs, $1 \mathrm{H}$ ), 9 (d, $\left.J=4.8 \mathrm{~Hz}, 1 \mathrm{H}\right), 7.97$ (dd, $J=4.9,1.2 \mathrm{~Hz}, 1 \mathrm{H}$ ), $7.36(\mathrm{t}, J=7.2 \mathrm{~Hz}, 2 \mathrm{H}), 7.3(\mathrm{~m}, 3 \mathrm{H}), 6.14(\mathrm{q}, J=9.2 \mathrm{~Hz}, 1 \mathrm{H}), 5.1(\mathrm{~s}, 2 \mathrm{H})$

${ }^{13} \mathrm{C}$ NMR $\left(100 \mathrm{MHz}\right.$, DMSO- $\left.d_{6}\right) \delta \mathrm{ppm} 165.1,152.9,145.4,142.7$ (q, $\left.J=6.3 \mathrm{~Hz}\right), 137.1,136,129.2(2 \mathrm{C})$, 128.0, 127.4 (2C), 127.3, $124.4(q, J=268.3 \mathrm{~Hz}), 117.7,97.2(q, J=37.6 \mathrm{~Hz}), 42.8$ 
${ }^{19}$ F NMR (376 MHz, dmso-d6) $\delta$ ppm -52.5

HRMS (ESI): calcd. for $\mathrm{C}_{16} \mathrm{H}_{12} \mathrm{~F}_{3} \mathrm{~N}_{2} \mathrm{O}\left([\mathrm{M}+\mathrm{H}]^{+}\right)$305.0896, found 305.0894

(3Z)-2-benzyl-3-(2,2,2-trifluoroethylidene)pyrrolo[3,4-c]pyridin-1-one $4 \mathrm{mZ}$ IR (neat): $1728,1662,1126-1100 \mathrm{~cm}^{-1}$

${ }^{1} \mathbf{H}$ NMR (400 MHz, DMSO-d $\left.d_{6}\right) \delta$ ppm $9.55(\mathrm{~s}, 1 \mathrm{H}), 9(\mathrm{~d}, J=4.9 \mathrm{~Hz}, 1 \mathrm{H}), 7.9(\mathrm{dd}, J=4.9,1.1 \mathrm{~Hz}, 1 \mathrm{H})$, $7.35(\mathrm{t}, J=7.5 \mathrm{~Hz}, 2 \mathrm{H}), 7.27(\mathrm{t}, J=7.4 \mathrm{~Hz}, 1 \mathrm{H}$ ), 7.11 (d, J = 7.4 Hz, $2 \mathrm{H}), 6.5$ (q, J=10.0 Hz $1 \mathrm{H}$ ), 5.17 (s, $2 \mathrm{H})$

${ }^{13} \mathrm{C}$ NMR $\left(100 \mathrm{MHz}\right.$, DMSO- $\left.d_{6}\right) \delta \mathrm{ppm} 167.4,152.5,144,140.5(\mathrm{q}, J=6.6 \mathrm{~Hz}), 136.5,134.3,131.9,129$ (2C), 127.5, $126(2 \mathrm{C}), 123$ (q, $J=268.9 \mathrm{~Hz}), 117.5,94(q, J=37.6 \mathrm{~Hz}), 45$

${ }^{19}$ F NMR (376 MHz, dmso-d6) $\delta$ ppm -49.2

HRMS (ESI): calcd. for $\mathrm{C}_{16} \mathrm{H}_{12} \mathrm{~F}_{3} \mathrm{~N}_{2} \mathrm{O}\left([\mathrm{M}+\mathrm{H}]^{+}\right)$305.0896, found 305.0896

\section{(6E/Z)-7-benzyl-6-(2,2,2-trifluoroethylidene)pyrrolo[3,4-g][1,3]benzothiazol-8- one $4 \mathrm{n}$ and (5E/Z)-6-benzyl-5-(2,2,2-trifluoroethylidene)pyrrolo[3,4- f][1,3]benzothiazol-7-one $4 n$ '}<smiles>O=C1c2c(ccc3ncsc23)C(=CC(F)(F)F)N1Cc1ccccc1</smiles>

Starting from N-benzyl-N-ethynyl-1,3-benzothiazole-6-carboxamide $3 \mathrm{n}$ (117 mg, $0.4 \mathrm{mmol}$ ), Togni I reagent $(158 \mathrm{mg}, 0.48 \mathrm{mmol})$ and $\operatorname{lr}(\mathrm{ppy})_{3}(6.6 \mathrm{mg}, 0.01 \mathrm{mmol})$, using General procedure 3 and stirring at $27^{\circ} \mathrm{C}$ for $22 \mathrm{~h}$. The crude was purified by silica gel flash column chromatography to afford (6E)-7-benzyl-6-(2,2,2-trifluoroethylidene)pyrrolo[3,4-g][1,3]benzothiazol-8-one $4 \mathrm{nE}$ as a white solid (33 mg), (6Z)-7-benzyl-6-(2,2,2-trifluoroethylidene)pyrrolo[3,4-g][1,3]benzothiazol-8-one $4 \mathrm{nz}$ as a white solid (19 mg) (E/Z mixture 63/37, $36 \%$ overall yield) and a mixture of (5E/Z)-6-benzyl-5-(2,2,2trifluoroethylidene)pyrrolo[3,4-f][1,3]benzothiazol-7-one $\mathbf{4 n}$ ' as a white solid (22 $\mathrm{mg}, 15 \%$ yield) (E/Z mixture 78/22).

(6E)-7-benzyl-6-(2,2,2-trifluoroethylidene)pyrrolo[3,4-g][1,3]benzothiazol-8-one 4nE IR (neat): 3070, 1724, 1652, 1099, 717, 692, $675 \mathrm{~cm}^{-1}$

${ }^{1} \mathbf{H}$ NMR $\left(500 \mathrm{MHz}\right.$, DMSO- $\left.d_{6}\right) \delta \mathrm{ppm} 9.79(\mathrm{~s}, 1 \mathrm{H}), 8.47(\mathrm{~d}, J=8.2 \mathrm{~Hz}, 1 \mathrm{H}), 8.06(\mathrm{~d}, J=8.2 \mathrm{~Hz}, 1 \mathrm{H})$, $7.31(\mathrm{t}, J=7.4 \mathrm{~Hz}, 2 \mathrm{H}), 7.23(\mathrm{t}, J=7.3 \mathrm{~Hz}, 1 \mathrm{H}), 7.14(\mathrm{~d}, J=7.5 \mathrm{~Hz}, 2 \mathrm{H}), 5.79(\mathrm{q}, J=9.6 \mathrm{~Hz}, 1 \mathrm{H}), 5.17$ $(\mathrm{s}, 2 \mathrm{H})$

${ }^{13} \mathrm{C}$ NMR $\left(125 \mathrm{MHz}, \mathrm{DMSO}-d_{6}\right) \delta \mathrm{ppm} 168,160.2,158.8,142.5$ (q, $\left.J=6.3 \mathrm{~Hz}\right), 136.8,132,128.9(2 \mathrm{C})$, $127.4,127.1,126.8,126.3$ (q, $J=268.7 \mathrm{~Hz}$ ), $125.8(2 \mathrm{C}), 125.5,121.7,94.5$ (q, J = 38.4 Hz), 45.2

${ }^{19}$ F NMR $\left(376 \mathrm{MHz}\right.$, DMSO- $\left.d_{6}\right) \delta \mathrm{ppm}-48.9$

HRMS (ESI): calcd. for $\mathrm{C}_{18} \mathrm{H}_{11} \mathrm{~F}_{3} \mathrm{O} \mathrm{S}\left([\mathrm{M}+\mathrm{H}]^{+}\right)$361.0617, found 361.0619 
mp: $171.7-174.9^{\circ} \mathrm{C}$

(6Z)-7-benzyl-6-(2,2,2-trifluoroethylidene)pyrrolo[3,4-g][1,3]benzothiazol-8-one 4nZ IR (neat): 3060, 1714, 1649, 1110, 777, 713, $678 \mathrm{~cm}^{-1}$

${ }^{1} \mathrm{H}$ NMR $\left(400 \mathrm{MHz}\right.$, DMSO- $\left.d_{6}\right) \delta \mathrm{ppm} 9.7(\mathrm{~s}, 1 \mathrm{H}), 8.52(\mathrm{~d}, J=8.2 \mathrm{~Hz}, 1 \mathrm{H}), 8.1(\mathrm{~d}, J=8.2 \mathrm{~Hz}, 1 \mathrm{H}), 7.37$ $(\mathrm{t}, J=7.4 \mathrm{~Hz}, 2 \mathrm{H}), 7.3(\mathrm{~m}, 3 \mathrm{H}), 6.09(\mathrm{q}, J=9.9 \mathrm{~Hz}, 1 \mathrm{H}), 5.16(\mathrm{~s}, 2 \mathrm{H})$

${ }^{13} \mathrm{C}$ NMR $\left(75 \mathrm{MHz}, \mathrm{DMSO}-d_{6}\right) \delta \mathrm{ppm} 166,159.8,158.6,143.1(\mathrm{q}, J=6.2 \mathrm{~Hz}), 136.4,130.2,129.3(2 \mathrm{C})$, $129,128.3(2 \mathrm{C}), 128.1,127.6,127.5,124.1(\mathrm{q}, J=267.3 \mathrm{~Hz}), 121.8,98.7(\mathrm{q}, J=38.0 \mathrm{~Hz}), 43$

${ }^{19}$ F NMR $\left(376 \mathrm{MHz}, \mathrm{DMSO}-d_{6}\right) \delta \mathrm{ppm}-46.8$

HRMS (ESI): calcd. for $\mathrm{C}_{18} \mathrm{H}_{11} \mathrm{~F}_{3} \mathrm{O} \mathrm{S}\left([\mathrm{M}+\mathrm{H}]^{+}\right)$361.0617, found 361.0618

$\mathrm{mp}: 181.5-185.7^{\circ} \mathrm{C}$

(5E)-6-benzyl-5-(2,2,2-trifluoroethylidene)pyrrolo[3,4-f][1,3]benzothiazol-7-one 4n'E IR (neat): 3076, 1724, 1651, 1132, 1099, 839, 732, $692 \mathrm{~cm}^{-1}$ (mixture 78/22 E/Z)

${ }^{1}$ H NMR $\left(500 \mathrm{MHz}, \mathrm{DMSO}-d_{6}\right) \delta \mathrm{ppm} 9.7(\mathrm{~s}, 1 \mathrm{H}), 8.86(\mathrm{~s}, 1 \mathrm{H}), 8.51(\mathrm{~s}, 1 \mathrm{H}), 7.36(\mathrm{t}, J=7.4 \mathrm{~Hz}, 2 \mathrm{H}), 7.3$ $(\mathrm{m}, 3 \mathrm{H}), 5.97(\mathrm{q}, J=9.5 \mathrm{~Hz}, 1 \mathrm{H}), 5.11(\mathrm{~s}, 2 \mathrm{H})$

${ }^{13} \mathrm{C}$ NMR $\left(125 \mathrm{MHz}\right.$, DMSO- $\left.d_{6}\right) \delta \mathrm{ppm} 165.9,161.9,156.5,144.1(\mathrm{q}, J=6.2 \mathrm{~Hz}), 137.8,136.7,129.5$ (2C), 129.5, $127.9(2 \mathrm{C}), 127.7,126.7,125.1$ (q, $J=268.9 \mathrm{~Hz}), 119.8,119.3,95.6$ (q, $J=37.7 \mathrm{~Hz}), 43.1$

${ }^{19}$ F NMR $\left(376 \mathrm{MHz}\right.$, DMSO-d $\left.d_{6}\right) \delta \mathrm{ppm}-52.1$

HRMS (ESI): calcd. for $\mathrm{C}_{18} \mathrm{H}_{11} \mathrm{~F}_{3} \mathrm{OS}\left([\mathrm{M}+\mathrm{H}]^{+}\right)$361.0617, found 361.0619

(5Z)-6-benzyl-5-(2,2,2-trifluoroethylidene)pyrrolo[3,4-f][1,3]benzothiazol-7-one 4n'Z

${ }^{1} \mathrm{H}$ NMR $\left(500 \mathrm{MHz}\right.$, DMSO- $\left.d_{6}\right) \delta \mathrm{ppm} 9.69(\mathrm{~s}, 1 \mathrm{H}), 9(\mathrm{~s}, 1 \mathrm{H}), 8.8(\mathrm{~s}, 1 \mathrm{H}), 7.32(\mathrm{~m}, 2 \mathrm{H}), 7.24(\mathrm{t}, J=7.3$ $\mathrm{Hz}, 1 \mathrm{H}), 7.1(\mathrm{~d}, J=7.5 \mathrm{~Hz}, 2 \mathrm{H}), 6.58(\mathrm{q}, J=10.1 \mathrm{~Hz}, 1 \mathrm{H}), 5.16(\mathrm{~s}, 2 \mathrm{H})$

${ }^{13} \mathrm{C}$ NMR $\left(125 \mathrm{MHz}, \mathrm{DMSO}-d_{6}\right) \delta \mathrm{ppm} 168.1,161.9,156.9,142$ (q, $\left.J=6.4 \mathrm{~Hz}\right), 137.6,137,135.1,127.4$ (2C), 127.4, $124.8(2 \mathrm{C}), 124.5,124$ (q, J = 268.2 Hz), 119.6, 116.7, 91.4 (q, J = 37.7 Hz), 45.2

${ }^{19}$ F NMR $\left(376 \mathrm{MHz}, \mathrm{DMSO}-d_{6}\right) \delta \mathrm{ppm}-48.5$

\section{(3E/Z)-2-benzyl-4,6-difluoro-3-(2,2,2-trifluoroethylidene)isoindolin-1-one 40}<smiles>O=C1c2cc(F)cc(F)c2/C(=C\C(F)(F)F)N1Cc1ccccc1</smiles>

Starting from N-benzyl-N-ethynyl-3,5-difluoro-benzamide 30 (54 mg, $0.2 \mathrm{mmol}$ ), Togni I reagent (79 $\mathrm{mg}, 0.24 \mathrm{mmol})$ and $\operatorname{Ir}(\mathrm{ppy})_{3}(3.3 \mathrm{mg}, 0.005 \mathrm{mmol})$, using General procedure 3 and stirring at $27^{\circ} \mathrm{C}$ for $12 \mathrm{~h}$. The crude was purified by silica gel flash column chromatography to afford (3E)-2-benzyl-4,6difluoro-3-(2,2,2-trifluoroethylidene)isoindolin-1-one $4 \mathrm{oE}$ as a colorless oil (22 mg) and (3Z)-2-benzyl- 
4,6-difluoro-3-(2,2,2-trifluoroethylidene)isoindolin-1-one $4 \mathrm{oZ}$ as a colorless oil (22 mg) (E/Z mixture $50 / 50,62 \%$ overall yield).

(3E)-2-benzyl-4,6-difluoro-3-(2,2,2-trifluoroethylidene)isoindolin-1-one 4oE IR (neat): 1720, 1658, 1336, $1112 \mathrm{~cm}^{-1}$

${ }^{1} \mathrm{H}$ NMR $\left(400 \mathrm{MHz}\right.$, DMSO- $\left.d_{6}\right) \delta \mathrm{ppm} 7.78(\mathrm{td}, J=10.2,2.3 \mathrm{~Hz}, 1 \mathrm{H}), 7.73(\mathrm{dd}, J=6.6,2.3 \mathrm{~Hz}, 1 \mathrm{H}), 7.36$ $(\mathrm{t}, J=7.4 \mathrm{~Hz}, 2 \mathrm{H}), 7.28(\mathrm{~m}, 3 \mathrm{H}), 6(\mathrm{q}, J=9.1 \mathrm{~Hz}, 1 \mathrm{H}), 5.08(\mathrm{~s}, 2 \mathrm{H})$

${ }^{13} \mathrm{C}$ NMR (75 MHz, DMSO- $d_{6}$ ) $\delta$ ppm 165.1 (dd, $\left.J=255.5,10.9 \mathrm{~Hz}\right), 164.8,157.5$ (dd, $J=262.3,13.0$ $\mathrm{Hz}, 139.7(\mathrm{bq}, J=5.7 \mathrm{~Hz}), 136.3,134.7,129.2(2 \mathrm{C}), 127.9,126.9(2 \mathrm{C}), 123.7(\mathrm{q}, J=267.7 \mathrm{~Hz}), 115.1$, $110.1(\mathrm{t}, J=27.6 \mathrm{~Hz}), 108.3(\mathrm{dd}, J=24.1,3.2 \mathrm{~Hz}), 99.4(\mathrm{q}, J=38.9 \mathrm{~Hz}), 42.6$

${ }^{19}$ F NMR $\left(376 \mathrm{MHz}\right.$, DMSO- $\left.d_{6}\right) \delta \mathrm{ppm}-50.8,-100.4,-102.2$

HRMS (EI): calcd. for $\mathrm{C}_{17} \mathrm{H}_{10} \mathrm{~F}_{5} \mathrm{~N} \mathrm{O}\left([\mathrm{M}]^{+}\right)$339.0677, found 339.067

(3Z)-2-benzyl-4,6-difluoro-3-(2,2,2-trifluoroethylidene)isoindolin-1-one 4oZ IR (neat): 3091, 3068, 3033, 1739, 1656, 1097, 761, 729, $692 \mathrm{~cm}^{-1}$

${ }^{1} \mathrm{H}$ NMR (400 MHz, DMSO- $\left.d_{6}\right) \delta$ ppm 7.84 (td, $\left.J=10.1,2.1 \mathrm{~Hz}, 1 \mathrm{H}\right), 7.73(\mathrm{dd}, J=9.9,2.1 \mathrm{~Hz}, 1 \mathrm{H}), 7.31$ $(\mathrm{t}, J=7.4 \mathrm{~Hz}, 2 \mathrm{H}), 7.24(\mathrm{t}, J=7.2 \mathrm{~Hz}, 1 \mathrm{H}), 7.11(\mathrm{~d}, J=7.5 \mathrm{~Hz}, 2 \mathrm{H}), 6.02\left(\mathrm{qd}^{7}, J=9.9,4.2 \mathrm{~Hz}, 1 \mathrm{H}\right), 5.11$ $(\mathrm{s}, 2 \mathrm{H})$

${ }^{13} \mathrm{C}$ NMR $\left(75 \mathrm{MHz}\right.$, DMSO- $\left.d_{6}\right) \delta \mathrm{ppm} 166.5,164.9$ (dd, $J=254.1,11.0 \mathrm{~Hz}$ ), 157.9 (dd, $J=258.5,13.0$ $\mathrm{Hz}), 138.8(\mathrm{bq}, J=6.4 \mathrm{~Hz}), 136.3,131.8,128.6(2 \mathrm{C}), 127.3,125.5(2 \mathrm{C}), 123.2(\mathrm{q}, J=268.7 \mathrm{~Hz}), 119.2$, $109.9(\mathrm{t}, J=26.4 \mathrm{~Hz}), 108.1(\mathrm{dd}, J=24.6,4.2 \mathrm{~Hz}), 95.4(\mathrm{bq}, J=37.8 \mathrm{~Hz}), 45.1$

${ }^{19}$ F NMR $\left(376 \mathrm{MHz}\right.$, DMSO- $\left.d_{6}\right) \delta \mathrm{ppm}-49.5,-103.2,-111.7$

HRMS (EI): calcd. for $\mathrm{C}_{17} \mathrm{H}_{10} \mathrm{~F}_{5} \mathrm{~N} \mathrm{O}\left([\mathrm{M}+\mathrm{H}]^{+}\right)$339.0677, found 339.0675

\section{(3E/Z)-2-benzyl-7-methoxy-3-(2,2,2-trifluoroethylidene)isoindolin-1-one 4p}<smiles>COc1cccc2c1C(=O)N(Cc1ccccc1)C2=O</smiles>

Starting from N-benzyl-N-ethynyl-2-methoxy-benzamide 3p (106 mg, $0.4 \mathrm{mmol}$ ), Togni I reagent (158 $\mathrm{mg}, 0.48 \mathrm{mmol})$ and $\operatorname{Ir}(\mathrm{ppy})_{3}(6.6 \mathrm{mg}, 0.01 \mathrm{mmol})$, using General procedure 3 and stirring at $27^{\circ} \mathrm{C}$ for $20 \mathrm{~h}$. The crude was purified by silica gel flash column chromatography to afford (3E)-2-benzyl-7methoxy-3-(2,2,2-trifluoroethylidene)isoindolin-1-one 4pE as a white solid (13 mg) and (3Z)-2-benzyl7-methoxy-3-(2,2,2-trifluoroethylidene)isoindolin-1-one $4 \mathrm{pZ}$ as a white solid (10 mg) (E/Z mixture $52 / 48,24 \%$ overall yield).

(3E)-2-benzyl-7-methoxy-3-(2,2,2-trifluoroethylidene)isoindolin-1-one 4pE

\footnotetext{
${ }^{7}$ Through space coupling
} 
IR (neat): 1724, 1643, 1292/1249, 1105, 758, $700 \mathrm{~cm}^{-1}$

${ }^{1}$ H NMR (400 MHz, DMSO-d $\left.d_{6}\right) \delta$ ppm $7.75(\mathrm{t}, J=7.9 \mathrm{~Hz}, 1 \mathrm{H}), 7.49(\mathrm{~d}, J=7.3 \mathrm{~Hz}, 1 \mathrm{H}), 7.45$ (d, $J=8.4$ $\mathrm{Hz}, 1 \mathrm{H}), 7.35(\mathrm{t}, J=7.4 \mathrm{~Hz}, 2 \mathrm{H}), 7.30-7.21(\mathrm{~m}, 3 \mathrm{H}), 5.68(\mathrm{q}, J=9.6 \mathrm{~Hz}, 1 \mathrm{H}), 5.05(\mathrm{~s}, 2 \mathrm{H}), 3.91(\mathrm{~s}, 3 \mathrm{H})$

${ }^{13}$ C NMR $\left(75 \mathrm{MHz}, \mathrm{DMSO}-d_{6}\right) \delta$ ppm 166.4, 156, 141.7 (q, $\left.J=6.6 \mathrm{~Hz}\right), 136.9,134.8,131.9,129.0(2 \mathrm{C})$, $127.7,127.2(2 \mathrm{C}), 121.2(\mathrm{q}, J=268.0 \mathrm{~Hz}), 119.4,117.6,115.8,98.1(q, J=39.3 \mathrm{~Hz}), 55.9,42.5$

${ }^{19}$ F NMR $\left(376 \mathrm{MHz}, \mathrm{DMSO}-d_{6}\right) \delta \mathrm{ppm}-48.7$

HRMS (ESI): calcd. for $\mathrm{C}_{18} \mathrm{H}_{15} \mathrm{~F}_{3} \mathrm{~N} \mathrm{O}_{2}\left([\mathrm{M}+\mathrm{H}]^{+}\right)$334.1049, found 334.105

$\mathrm{mp}: 124.0-127.1^{\circ} \mathrm{C}$

(3Z)-2-benzyl-7-methoxy-3-(2,2,2-trifluoroethylidene)isoindolin-1-one 4pZ IR (neat): 1726, 1651, 1247, 1080, 748, 731, $694 \mathrm{~cm}^{-1}$

${ }^{1} \mathbf{H}$ NMR (400 MHz, DMSO- $\left.d_{6}\right) \delta \mathrm{ppm} 7.71(\mathrm{t}, J=7.8 \mathrm{~Hz}, 1 \mathrm{H}), 7.50(\mathrm{~m}, 2 \mathrm{H}), 7.3(\mathrm{t}, J=7.5 \mathrm{~Hz}, 2 \mathrm{H}), 7.22$ $(\mathrm{t}, J=7.4 \mathrm{~Hz}, 1 \mathrm{H}), 7.06(\mathrm{~d}, J=7.7 \mathrm{~Hz}, 2 \mathrm{H}), 6.5(\mathrm{q}, J=10.5 \mathrm{~Hz}, 1 \mathrm{H}), 6.1(\mathrm{~s}, 2 \mathrm{H}), 4.04(\mathrm{~s}, 3 \mathrm{H})$

${ }^{13} \mathrm{C}$ NMR $\left(75 \mathrm{MHz}\right.$, DMSO- $\left.d_{6}\right) \delta \mathrm{ppm} 168.1,156,141.5(\mathrm{q}, J=6.8 \mathrm{~Hz}), 136.8,133.6,132.7(\mathrm{q}, J=267.2$ $\mathrm{Hz}), 129.2,127.8(2 \mathrm{C}), 127.5,126.4(2 \mathrm{C}), 122,117.4,116,94.6$ (q, $J=37.6 \mathrm{~Hz}), 56.8,44.7$

${ }^{19}$ F NMR $\left(376 \mathrm{MHz}, \mathrm{DMSO}-d_{6}\right) \delta \mathrm{ppm}-48.6$

HRMS (ESI): calcd. for $\mathrm{C}_{18} \mathrm{H}_{15} \mathrm{~F}_{3} \mathrm{~N} \mathrm{O}_{2}\left([\mathrm{M}+\mathrm{H}]^{+}\right) 334.1049$, found 334.1049

$m p: 135.4-136.8^{\circ} \mathrm{C}$

(3E/Z)-2-(3-methoxypropyl)-3-(2,2,2-trifluoroethylidene)isoindolin-1-one 4q<smiles>COCCCN1C(=O)c2ccccc2/C1=C\C(F)(F)F</smiles>

Starting from N-ethynyl-N-(3-methoxypropyl)benzamide $3 \mathbf{q}(80 \mathrm{mg}, 0.37 \mathrm{mmol})$, Togni I reagent (146 $\mathrm{mg}, 0.44 \mathrm{mmol})$ and $\operatorname{Ir}(\mathrm{ppy})_{3}(6.1 \mathrm{mg}, 0.009 \mathrm{mmol})$, using General procedure 3 and stirring at $27^{\circ} \mathrm{C}$ for $2.7 \mathrm{~h}$. The crude was purified by silica gel flash column chromatography to afford a mixture of (3E/Z)2-(3-methoxypropyl)-3-(2,2,2-trifluoroethylidene)isoindolin-1-one $\mathbf{4 q}$ as a beige solid $(60 \mathrm{mg}, 57 \%$ yield) (E/Z mixture 76/24).

(3E)-2-(3-methoxypropyl)-3-(2,2,2-trifluoroethylidene)isoindolin-1-one 4qE IR (neat): $1728,1655 \mathrm{~cm}^{-1}$ (mixture 78/22 E/Z)

${ }^{1} \mathrm{H}$ NMR (500 MHz, DMSO-d $\left.d_{6}\right) \delta \mathrm{ppm} 7.96(\mathrm{~d}, J=7.8 \mathrm{~Hz}, 1 \mathrm{H}), 7.86(\mathrm{~d}, J=7.4 \mathrm{~Hz}, 1 \mathrm{H}), 7.81(\mathrm{~m}, 1 \mathrm{H})$, $7.73(\mathrm{t}, J=7.5 \mathrm{~Hz}, 1 \mathrm{H}), 6.04(\mathrm{q}, J=9.5 \mathrm{~Hz}, 1 \mathrm{H}), 3.82(\mathrm{t}, J=7 \mathrm{~Hz}, 2 \mathrm{H}), 3.35(\mathrm{~m}, 2 \mathrm{H}), 3.22(\mathrm{~s}, 3 \mathrm{H}), 1.81$ $(\mathrm{m}, 2 \mathrm{H})$

${ }^{13} \mathrm{C}$ NMR $\left(100 \mathrm{MHz}, \mathrm{DMSO}-d_{6}\right) \delta \mathrm{ppm} 166.3,144.4(\mathrm{q}, J=6.2 \mathrm{~Hz}), 133.7,132,131.9,130.1,125.2$ (q, $J$ $=267.9 \mathrm{~Hz}), 124.5,123.5,94.8(q, J=37.6 \mathrm{~Hz}), 69.6,58.3,36.8,28.1$ 
${ }^{19}$ F NMR (376 MHz, DMSO- $\left.d_{6}\right) \delta$ ppm -51.6

HRMS (ESI): calcd. for $\mathrm{C}_{14} \mathrm{H}_{15} \mathrm{~F}_{3} \mathrm{~N} \mathrm{O}_{2}([\mathrm{M}+\mathrm{H}]+)$ 286.1049, found 286.1051

(3Z)-2-(3-methoxypropyl)-3-(2,2,2-trifluoroethylidene)isoindolin-1-one 4qZ

${ }^{1} \mathbf{H}$ NMR (500 MHz, DMSO- $\left.d_{6}\right) \delta \mathrm{ppm} 8.15(\mathrm{~d}, J=7.6 \mathrm{~Hz}, 1 \mathrm{H}), 7.81(\mathrm{~m}, 1 \mathrm{H}), 7.76(\mathrm{t}, J=7.6 \mathrm{~Hz}, 1 \mathrm{H})$, $7.68(\mathrm{t}, J=7.4 \mathrm{~Hz}, 1 \mathrm{H}), 6.29(\mathrm{q}, J=10.2 \mathrm{~Hz}, 1 \mathrm{H}), 3.88(\mathrm{t}, J=7.6 \mathrm{~Hz}, 2 \mathrm{H}), 3.35(\mathrm{~m}, 2 \mathrm{H}), 3.21(\mathrm{~s}, 3 \mathrm{H})$, $1.8(\mathrm{~m}, 2 \mathrm{H})$

${ }^{13} \mathrm{C}$ NMR $\left(100 \mathrm{MHz}\right.$, DMSO- $\left.d_{6}\right) \delta \mathrm{ppm} 168.4,142.1$ (q, $\left.J=6.5 \mathrm{~Hz}\right), 137,133.3,132,127.4,124.2(\mathrm{q}, J=$ $267.9 \mathrm{~Hz}), 123,121.7,91.1(\mathrm{q}, J=37.6 \mathrm{~Hz}), 69.7,58.3,39.5,28.1$

${ }^{19}$ F NMR $\left(376 \mathrm{MHz}, \mathrm{DMSO}-d_{6}\right) \delta \mathrm{ppm}-49$ 


\section{$\mathrm{X}$-Ray crystal structure determination of compounds $4 \mathrm{aE}$ and $4 \mathrm{aZ}$}

A single crystal of each compound (4aE and $4 \mathrm{aZ}$ ) was selected, mounted onto a cryoloop and transferred into a cold nitrogen gas stream. Intensity data were collected with a Bruker Kappa-APEXII diffractometer using graphite-monochromated Mo-Ka radiation $(\lambda=0.71073 \AA$ $)$. Data collection was performed with the Bruker APEXII suite. Unit-cell parameters determination, integration and data reduction were carried out with SAINT program. SADABS was used for scaling and absorption corrections. The structures were solved with SHELXS-2014 ${ }^{1}$ for $4 \mathrm{aZ}$ or SHELXT-2014 $4^{2}$ for $4 \mathrm{aE}$, and refined by full-matrix least-squares methods with SHELXL-2014 ${ }^{3}$ using the Olex 2 software package ${ }^{4}$. All non-hydrogen atoms were refined anisotropically. The structures were deposited at the Cambridge Crystallographic Data Centre with numbers CCDC 1949295 to 1949296 and can be obtained free of charge via www.ccdc.cam.ac.uk.

X-Ray crystal structure of compound $4 \mathrm{aE}$ (E isomer) 


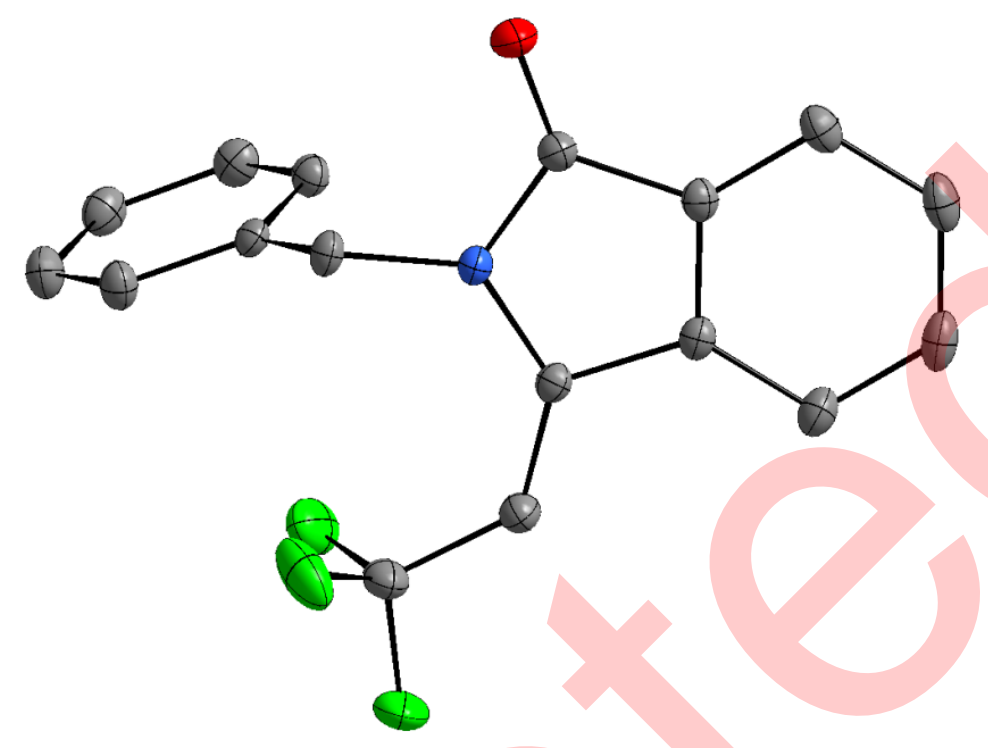

X-Ray crystal structure of compound $\mathbf{4 a Z}$ (Z isomer) 


\section{Crystal data and structure refinement for $4 \mathrm{aE}$}

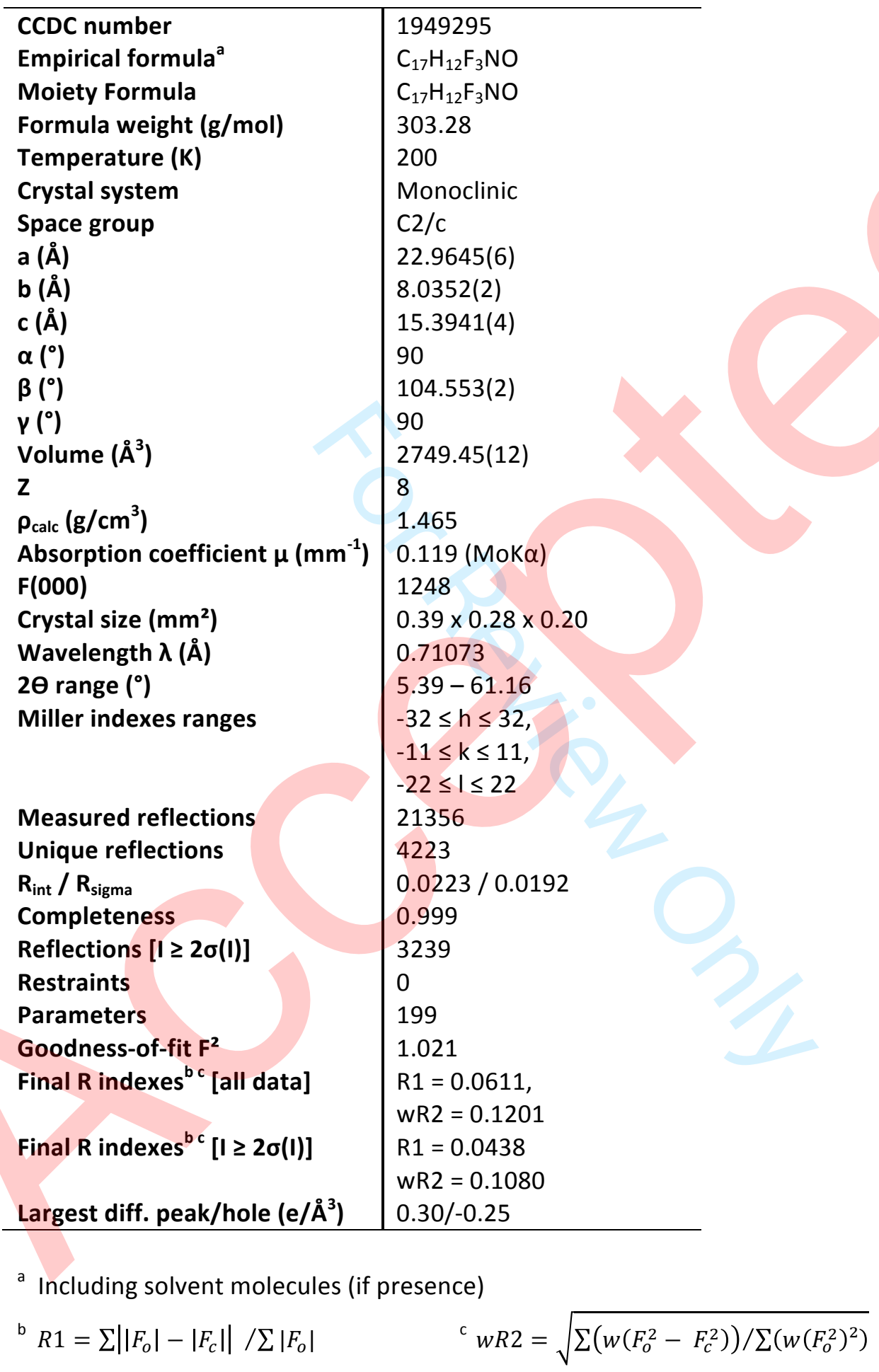




\section{Crystal data and structure refinement for $4 a z$}

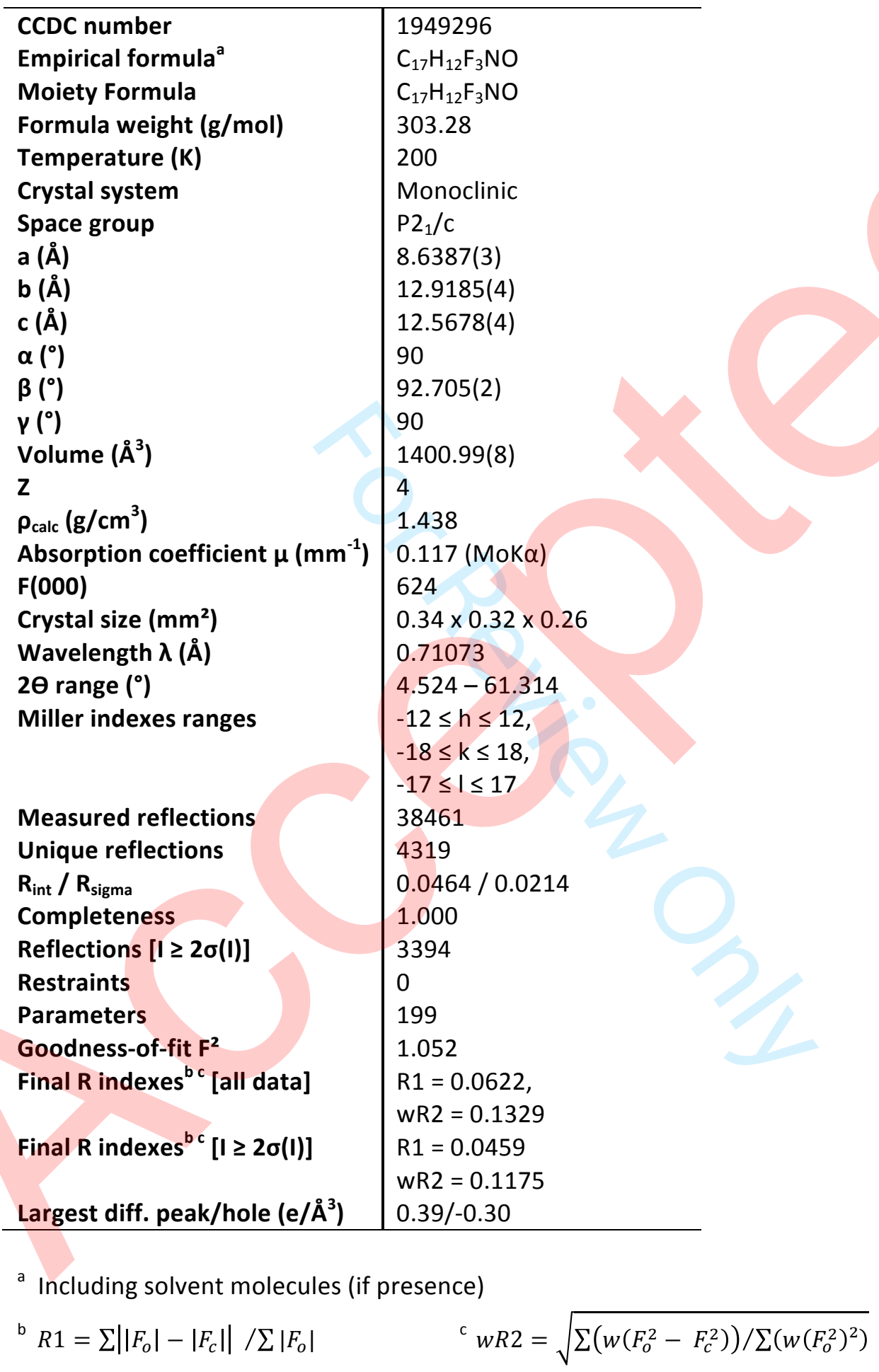




\section{DFT calculation method, energetics data and cartesian coordinates}

All calculations were performed using the Gaussian 09 software package ${ }^{8}$. Optimization of geometries, frequency and single point energy calculations were done using the PBEO functional including the D3BJ dispersion term ${ }^{9,10}$ with the def2-TZVP basis set ${ }^{11}$. The effect of solvation (in $\mathrm{MeCN}$ ) was modeled using the 'SMD' model ${ }^{12}$. The nature of all stationary points was confirmed by analyzing the harmonic vibrational frequencies.

Table 1. Energetics data $(\mathrm{kcal} / \mathrm{mol})$ relative to Scheme 5 obtained at the PBEO-D3BJ/def2-TZVP level of theory.

\begin{tabular}{|l|r|r|r|r|}
\hline $\begin{array}{l}\text { PBE0-D3BJ/ } \\
\text { Def2-TZVP }\end{array}$ & $\Delta \mathrm{E}$ & $\Delta \mathrm{E}+\mathrm{ZPE}$ & $\Delta \mathrm{G}$ & $\Delta \mathrm{G}_{\mathrm{MeCN}}$ \\
\hline $\mathbf{A}_{\mathrm{E}}{ }^{a}$ & 0.0 & 0.0 & 0.0 & 0.0 \\
\hline $\mathbf{A}_{\mathrm{Z}}{ }^{a}$ & 0.4 & 0.8 & 1.3 & 2.1 \\
\hline $\mathbf{T S}_{\mathrm{Ebz}}{ }^{a}$ & 5.5 & 4.9 & 6.3 & 5.7 \\
\hline $\mathbf{B}_{\mathrm{Ebz}}{ }^{a}$ & -26.1 & -25.4 & -23.5 & -26.1 \\
\hline $\mathbf{T S}_{\mathrm{Eb}}{ }^{a}$ & 12.3 & 10.9 & 13.5 & 12.8 \\
\hline $\mathbf{B}_{\mathrm{Eb}}{ }^{a}$ & -12.5 & -12.2 & -9.8 & -12.4 \\
\hline $\mathbf{T S}_{\mathrm{Zbz}}{ }^{b}$ & 9.4 & 8.4 & 9.1 & 6.9 \\
\hline $\mathbf{B}_{\mathrm{Zbz}}{ }^{b}$ & -21.8 & -21.6 & -20.2 & -24.9 \\
\hline $\mathbf{T S}_{\mathrm{Zb}}{ }^{b}$ & 13.7 & 12.7 & 14.8 & 12.0 \\
\hline $\mathbf{B}_{\mathrm{Zb}}{ }^{b}$ & -10.6 & -10.7 & -8.3 & -13.1 \\
\hline${ }^{2}$ & & &
\end{tabular}

$a$ energies relative to $\mathbf{A}_{E} ; b$ energies relative to $A_{Z}$

\footnotetext{
${ }^{8}$ Gaussian 09, Revision D.01, Frisch MJ, Trucks GW, Schlegel HB, Scuseria GE, Robb MA, Cheeseman JR, Scalmani G, Barone V, Mennucci B, Petersson GA, Nakatsuji H, Caricato M, Li X, Hratchian HP, Izmaylov AF, Bloino J, Zheng G, Sonnenberg JL, Hada M, Ehara M, Toyota K, Fukuda R, Hasegawa J, Ishida M, Nakajima T, Honda Y, Kitao O, Nakai H, Vreven T, Montgomery JA, Jr. Peralta JE, Ogliaro F, Bearpark M, Heyd JJ, Brothers E, Kudin KN, Staroverov VN, Kobayashi R, Normand J, Raghavachari K, Rendell A, Burant JC, lyengar SS, Tomasi J, Cossi M, Rega N, Millam JM, Klene M, Knox JE, Cross JB, Bakken V, Adamo C, Jaramillo J, Gomperts R, Stratmann RE, Yazyev O, Austin AJ, Cammi R, Pomelli C, Ochterski JW, Martin RL, Morokuma K, Zakrzewski VG, Voth GA, Salvador P, Dannenberg JJ, Dapprich S, Daniels AD, Farkas Ö, Foresman JB, Ortiz JV, Cioslowski J, Fox DJ. Gaussian, Inc., Wallingford CT, 2009

${ }^{9}$ Perdew JP, Burke K, Ernzerhof M. Phys. Rev. Lett. 1996, 77, 3865-3868

${ }^{10}$ Adamo C, Barone V. J. Chem. Phys. 1999, 110, 6158

${ }^{11}$ Weigend F, Ahlrichs R, Chem. Phys., 2005, 7, 3297

${ }^{12}$ Marenich AV, Cramer CJ, Truhlar DG. J. Phys. Chem. 2009,113, 6378
} 
Cartesian coordinates of all compounds involved in this work

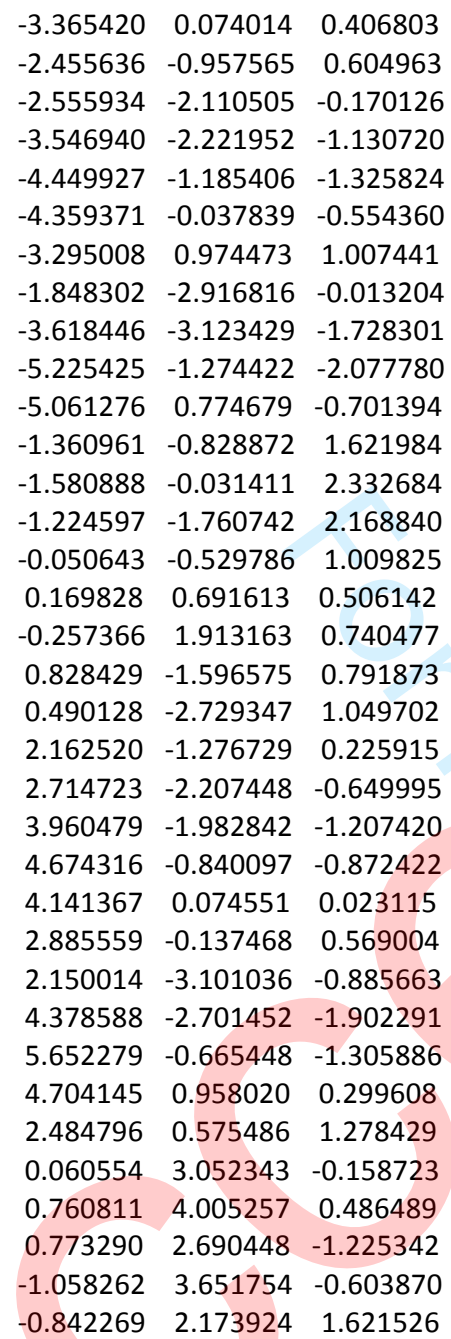

$\begin{array}{rrr}2.421950 & -0.840678 & 0.234932 \\ 3.121130 & -1.994297 & -0.108972 \\ 4.369331 & -1.899711 & -0.697597 \\ 4.939089 & -0.653010 & -0.919133 \\ 4.258681 & 0.497550 & -0.550049 \\ 2.998804 & 0.407741 & 0.019852 \\ 2.670098 & -2.958282 & 0.092023 \\ 4.903437 & -2.799567 & -0.978826 \\ 5.920501 & -0.578911 & -1.373252 \\ 4.710691 & 1.470332 & -0.702955 \\ 2.479370 & 1.308777 & 0.319569 \\ -0.706135 & 2.962914 & -0.056699 \\ -2.051832 & 3.049196 & -0.032969 \\ -0.310723 & 2.824736 & 1.216832 \\ -0.258398 & 4.148325 & -0.488353\end{array}$

TS $_{\mathrm{Ebz}}$ Frequencies -- -367.5841

\begin{tabular}{llrr}
6 & 3.560159 & 0.378455 & -0.638144 \\
6 & 2.573951 & -0.589361 & -0.499780 \\
6 & 2.578946 & -1.405438 & 0.627073 \\
6 & 3.552536 & -1.249382 & 1.599208 \\
6 & 4.535507 & -0.279434 & 1.454690 \\
6 & 4.539602 & 0.533104 & 0.332415 \\
1 & 3.560422 & 1.020520 & -1.513559 \\
1 & 1.817907 & -2.170308 & 0.737178 \\
1 & 3.546581 & -1.889314 & 2.474002 \\
1 & 5.295665 & -0.157921 & 2.217480 \\
1 & 5.302309 & 1.293860 & 0.213252 \\
6 & 1.526337 & -0.763507 & -1.563875 \\
1 & 1.609161 & 0.030838 & -2.310010 \\
1 & 1.643674 & -1.727767 & -2.062991 \\
7 & 0.164645 & -0.755043 & -1.045042 \\
6 & -0.421359 & 0.378294 & -0.589935 \\
6 & 0.062502 & 1.586402 & -0.361963 \\
6 & -0.548869 & -1.951425 & -0.949279 \\
8 & -0.073948 & -3.025425 & -1.228997 \\
6 & -1.882117 & -1.683028 & -0.379976 \\
6 & -2.387751 & -2.493409 & 0.614783 \\
6 & -3.461786 & -2.055086 & 1.382012 \\
6 & -4.004450 & -0.791921 & 1.156591 \\
6 & -3.528009 & 0.015517 & 0.141570 \\
6 & -2.448968 & -0.413323 & -0.651293 \\
1 & -1.903064 & -3.442065 & 0.814197 \\
1 & -3.858257 & -2.684222 & 2.169465 \\
1 & -4.833521 & -0.449440 & 1.765261 \\
1 & -3.997468 & 0.967912 & -0.066266 \\
1 & -2.326984 & 0.039743 & -1.630738 \\
6 & -0.776232 & 2.745259 & 0.032719 \\
9 & -2.042400 & 2.657232 & -0.403349 \\
9 & -0.843049 & 2.913368 & 1.365928 \\
9 & -0.266665 & 3.884967 & -0.464251 \\
1 & 1.131674 & 1.786473 & -0.385847 \\
& & & \\
\hline
\end{tabular}

$\begin{array}{lrrr}\mathbf{B}_{\text {Ebz }} & & & \\ 6 & 3.565688 & 0.115946 & -0.658995 \\ 6 & 2.476641 & -0.713236 & -0.425428 \\ 6 & 2.374448 & -1.368403 & 0.796495 \\ 6 & 3.345850 & -1.193337 & 1.768215 \\ 6 & 4.432641 & -0.363865 & 1.528304 \\ 6 & 4.542162 & 0.289416 & 0.311023 \\ 1 & 3.649737 & 0.635124 & -1.608863 \\ 1 & 1.529977 & -2.022131 & 0.985814\end{array}$


$\mathrm{TS}_{\mathrm{Eb}}$ Frequencies -- -405.3270

$\begin{array}{llll}6 & 2.493770 & -0.380783 & -0.682944 \\ 6 & 2.188027 & -1.707646 & -0.291212 \\ 6 & 2.780906 & -2.256753 & 0.822448 \\ 6 & 3.721632 & -1.526135 & 1.548422 \\ 6 & 4.031559 & -0.221877 & 1.173599 \\ 6 & 3.444176 & 0.346982 & 0.061178 \\ 1 & 2.296817 & -0.066298 & -1.704176 \\ 1 & 2.491255 & -3.249119 & 1.151148 \\ 1 & 4.195084 & -1.968376 & 2.416801 \\ 1 & 4.757028 & 0.344207 & 1.746243 \\ 1 & 3.715257 & 1.346682 & -0.252121 \\ 6 & 0.960471 & -2.255378 & -0.926277 \\ 1 & 1.051838 & -2.363318 & -2.012053 \\ 1 & 0.646940 & -3.213769 & -0.517958 \\ 7 & -0.075191 & -1.246676 & -0.611810 \\ 6 & 0.376183 & 0.031602 & -0.516024 \\ 6 & -0.159348 & 1.238362 & -0.569884 \\ 6 & -1.400344 & -1.678166 & -0.664384 \\ 8 & -1.665512 & -2.735881 & -1.189110 \\ 6 & -2.436649 & -0.843508 & -0.006617 \\ 6 & -2.223648 & -0.268813 & 1.242517 \\ 6 & -3.244806 & 0.429164 & 1.863453 \\ 6 & -4.473042 & 0.572590 & 1.232380 \\ 6 & -4.688090 & -0.003144 & -0.011743 \\ 6 & -3.676622 & -0.724228 & -0.624042 \\ 1 & -1.259574 & -0.369414 & 1.726331 \\ 1 & -3.081094 & 0.866748 & 2.841055 \\ 1 & -5.266774 & 1.130886 & 1.715138 \\ 1 & -5.648716 & 0.102835 & -0.501697 \\ 1 & -3.834690 & -1.204101 & -1.582393 \\ 6 & 0.652760 & 2.474532 & -0.415196 \\ 9 & -0.121607 & 3.558095 & -0.564930 \\ 9 & 1.246512 & 2.582679 & 0.786833 \\ 9 & 1.644728 & 2.579911 & -1.324805\end{array}$

$\begin{array}{lrrr}6 & 1.921892 & -0.217183 & -0.643206 \\ 6 & 2.366934 & -1.581360 & -0.209283 \\ 6 & 3.665304 & -1.886927 & 0.025627 \\ 6 & 4.657631 & -0.891989 & -0.069570 \\ 6 & 4.277304 & 0.448688 & -0.290186 \\ 6 & 2.987194 & 0.815015 & -0.507050 \\ 1 & 1.651556 & -0.274370 & -1.721788 \\ 1 & 3.936034 & -2.890104 & 0.339702 \\ 1 & 5.696351 & -1.141138 & 0.103326 \\ 1 & 5.041081 & 1.218320 & -0.269600 \\ 1 & 2.741898 & 1.850911 & -0.684779 \\ 6 & 1.165707 & -2.419833 & 0.045734 \\ 1 & 0.943246 & -3.135797 & -0.753656 \\ 1 & 1.231495 & -2.987777 & 0.977015 \\ 7 & 0.093501 & -1.426200 & 0.116675 \\ 6 & 0.576005 & -0.114080 & 0.057477 \\ 6 & -0.069863 & 0.930882 & 0.578334 \\ 1 & -0.992999 & 0.778509 & 1.118568 \\ 6 & -1.191409 & -1.895536 & 0.304756 \\ 8 & -1.360285 & -3.045646 & 0.652910 \\ 6 & -2.346135 & -1.011994 & -0.013247 \\ 6 & -3.453249 & -1.048642 & 0.825605 \\ 6 & -4.572140 & -0.286194 & 0.531169 \\ 6 & -4.600248 & 0.488883 & -0.618779 \\ 6 & -3.507703 & 0.500929 & -1.475486 \\ 6 & -2.379727 & -0.239841 & -1.170120 \\ 1 & -3.425126 & -1.680893 & 1.704964 \\ 1 & -5.427327 & -0.303675 & 1.196396 \\ 1 & -5.477420 & 1.080977 & -0.852452 \\ 1 & -3.534001 & 1.093747 & -2.382097 \\ 1 & -1.522754 & -0.220371 & -1.832901 \\ 6 & 0.290182 & 2.362351 & 0.428352 \\ 9 & 0.613039 & 2.700364 & -0.837353 \\ 9 & -0.753261 & 3.128768 & 0.775516 \\ 9 & 1.322406 & 2.753227 & 1.197008\end{array}$

$\mathrm{TS}_{\mathrm{Zbz}}$ Frequencies -- -338.5570

$\begin{array}{llll}6 & 1.597330 & -1.261953 & 0.859718 \\ 6 & 2.005406 & -0.910154 & -0.417484 \\ 6 & 3.315683 & -1.172296 & -0.802731 \\ 6 & 4.202328 & -1.773923 & 0.073767 \\ 6 & 3.788161 & -2.124051 & 1.351285 \\ 6 & 2.483909 & -1.866111 & 1.739590 \\ 1 & 0.582590 & -1.059909 & 1.182294 \\ 1 & 3.644995 & -0.903478 & -1.801680 \\ 1 & 5.220075 & -1.972223 & -0.242138 \\ 1 & 4.479935 & -2.596028 & 2.039031 \\ 1 & 2.150054 & -2.134323 & 2.735337 \\ 6 & 1.088154 & -0.246321 & -1.412518 \\ 1 & 1.441057 & 0.757310 & -1.651580 \\ 1 & 1.077742 & -0.829395 & -2.335763 \\ 7 & -0.296806 & -0.147718 & -0.974016 \\ 6 & -0.821298 & 0.898009 & -0.305811 \\ 6 & -0.509291 & 2.080926 & 0.190860 \\ 1 & -1.298232 & 2.672440 & 0.642581 \\ 6 & -1.119735 & -1.273661 & -1.158123 \\ 8 & -0.726815 & -2.283002 & -1.682050 \\ 6 & -2.440968 & -1.026424 & -0.549648 \\ 6 & -3.021396 & -1.963278 & 0.279384 \\ 6 & -4.069904 & -1.586854 & 1.113647 \\ 6 & -4.511615 & -0.266752 & 1.123174\end{array}$




\begin{abstract}
$0.672262 \quad 0.270536$
$\begin{array}{ll}0.308612 & -0.580208\end{array}$

$\begin{array}{ll}-2.967755 & 0.304274\end{array}$

$-2.3159301 .772357$

$0.021384 \quad 1.783788$

$\begin{array}{ll}1.678270 & 0.232621\end{array}$

$0.933700-1.445055$

$2.746090 \quad 0.195463$

1.9949120 .727826

$3.077647-1.044933$

3.8840240 .898556
\end{abstract}

B$$
6
$$$$
6
$$$$
6
$$

$-1.624781 \quad 0.745710$

1.419090

1.911294

3.285638

4.152568

3.654233

2.286091

0.351527

3.680919

5.221692

4.330874

1.886448

1.010880

1.406471

0.977965

$-2.841231$

$-2.881807$

$-3.533409$

$-4.199258$

$-4.167593$

$-3.519638$

$-2.647216$

$-3.497305$

$-4.723005$

$-4.680658$

$-3.545219$

$-1.834164$

$-1.951517$

$-1.754770$

$-0.586311$

$-0.714139$

0.016154

$-0.417828$

0.525161

0.601773

1.520265

1.136168

2.086962

3.423030

3.806890

2.856167

0.093009

1.784205

4.167600

4.849915

3.133818

1.393411

1.469513

1.758502

2.332111 $\begin{array}{ll}-0.985704 & -0.381705\end{array}$

$\begin{array}{ll}-0.947528 & -0.589234\end{array}$

$-1.5393850 .313226$

$\begin{array}{ll}-2.177079 & 1.440728\end{array}$

$\begin{array}{ll}-2.216088 & 1.653579\end{array}$

$\begin{array}{ll}-1.664482 & 0.926900\end{array}$

$-0.439823-1.463544$

$\begin{array}{lll}-1.497365 & 0.139562\end{array}$

$\begin{array}{ll}-2.637916 & 2.150800\end{array}$

$-2.710454 \quad 2.531565$

$-0.356869-1.415855$

$0.606140-1.733538$

$-1.008281-2.293508$

$\begin{array}{lll}0.901798 & 0.122722\end{array}$

$-0.354954 \quad 0.781322$

$\begin{array}{lll}-1.419406 & 0.203390\end{array}$

$\begin{array}{lll}-1.259882 & -1.013444\end{array}$

$-0.032280-1.670237$

$1.048871-1.105630$

$\begin{array}{lll}1.796207 & 0.708645\end{array}$

$\begin{array}{ll}-2.393982 & 0.678751\end{array}$

$-2.098594-1.455973$

$0.082556-2.618200$

$2.016762-1.592491$

$-0.496154 \quad 1.831979$

$\begin{array}{ll}0.221825 & 2.649786\end{array}$

$-1.493953 \quad 2.256245$

$\begin{array}{ll}-0.204012 & 1.089042\end{array}$

$0.773435 \quad 0.165574$

$\begin{array}{lll}1.721953 & -0.390120\end{array}$

$2.319475-1.185210$

$-1.016549 \quad 1.344143$

$\begin{array}{ll}-1.607432 & 2.395590\end{array}$

$\begin{array}{ll}-1.195891 & 0.259137\end{array}$

$-1.325435-1.071170$

$-1.594302-2.041141$

$-1.715535-1.689093$

$\begin{array}{ll}-1.592776 & -0.360685\end{array}$

$\begin{array}{ll}-1.348932 & 0.613707\end{array}$

$-1.229539-1.347371$

$-1.706107-3.075505$

$-1.911906-2.451948$

$-1.692333-0.084652$

$-1.268521 \quad 1.657438$

$2.141237-0.005657$

3.4833410 .035502

$1.690355 \quad 1.201636$

$1.741243-0.878949$

$\begin{array}{llrc}7 & -0.363739 & -0.197864 & -1.002545 \\ 6 & -0.931803 & 0.859193 & -0.326176 \\ 6 & -0.428145 & 2.012063 & 0.132676 \\ 1 & -1.124943 & 2.686946 & 0.610082 \\ 6 & -1.252453 & -1.269106 & -1.216149 \\ 8 & -0.950412 & -2.287938 & -1.788696 \\ 6 & -2.499523 & -0.886190 & -0.572193 \\ 6 & -3.510776 & -1.694242 & -0.144061 \\ 6 & -4.420425 & -1.193618 & 0.795823 \\ 6 & -4.195018 & 0.070954 & 1.401208 \\ 6 & -3.200496 & 0.900195 & 0.995332 \\ 6 & -2.416736 & 0.550289 & -0.218665 \\ 1 & -3.544952 & -2.730274 & -0.462237 \\ 1 & -5.232566 & -1.816019 & 1.149340 \\ 1 & -4.821930 & 0.364068 & 2.235899 \\ 1 & -3.037236 & 1.853702 & 1.482671 \\ 1 & -2.869992 & 1.122966 & -1.056313 \\ 6 & 0.945533 & 2.568228 & 0.062186 \\ 9 & 1.883751 & 1.794276 & 0.623309 \\ 9 & 1.362934 & 2.805199 & -1.203247 \\ 9 & 0.982813 & 3.749120 & 0.696730\end{array}$

$\mathrm{TS}_{\mathrm{Zb}}$ Frequencies -- -412.0166

$\begin{array}{lrrr}\mathbf{B}_{\text {Zb }} & & & \\ 6 & 2.364082 & 0.783511 & 0.032339 \\ 6 & 2.848393 & -0.390532 & -0.749780 \\ 6 & 3.908373 & -1.134812 & -0.354237 \\ 6 & 4.525885 & -0.870864 & 0.887453 \\ 6 & 3.938197 & 0.050462 & 1.783160 \\ 6 & 2.865281 & 0.808419 & 1.434819 \\ 1 & 2.729726 & 1.705929 & -0.464447 \\ 1 & 4.240432 & -1.978031 & -0.951106 \\ 1 & 5.383693 & -1.452452 & 1.199376 \\ 1 & 4.341767 & 0.132705 & 2.786427 \\ 1 & 2.416064 & 1.498461 & 2.139352 \\ 6 & 1.841887 & -0.656355 & -1.812673 \\ 1 & 1.991767 & -0.045173 & -2.712305 \\ 1 & 1.768469 & -1.697526 & -2.120701 \\ 7 & 0.608279 & -0.254050 & -1.120704 \\ 6 & 0.858711 & 0.770945 & -0.221108 \\ 6 & 0.054962 & 1.717307 & 0.272176 \\ 1 & 0.489811 & 2.416720 & 0.975197 \\ 6 & -0.540960 & -0.999179 & -1.353286 \\ 8 & -0.668843 & -1.589070 & -2.402696 \\ 6 & -1.508992 & -1.165144 & -0.239312 \\ 6 & -1.089180 & -1.292778 & 1.080086 \\ 6 & -2.013428 & -1.554033 & 2.077950 \\ 6 & -3.358952 & -1.671563 & 1.763728 \\ 6 & -3.778813 & -1.553060 & 0.445527 \\ 6 & -2.854925 & -1.315286 & -0.555274 \\ 1 & -0.038236 & -1.202947 & 1.327458 \\ 1 & -1.682184 & -1.663710 & 3.103842 \\ 1 & -4.082778 & -1.862428 & 2.547708 \\ 1 & -4.829440 & -1.650803 & 0.199085 \\ 1 & -3.160289 & -1.236746 & -1.591319 \\ 6 & -1.338977 & 2.067030 & -0.091939 \\ 9 & -1.450319 & 3.407427 & -0.166330 \\ 9 & -1.718285 & 1.582647 & -1.282689 \\ 9 & -2.254203 & 1.672426 & 0.807924\end{array}$




\section{${ }^{1} \mathrm{H},{ }^{13} \mathrm{C}$ and ${ }^{19} \mathrm{~F}$ NMR spectra}

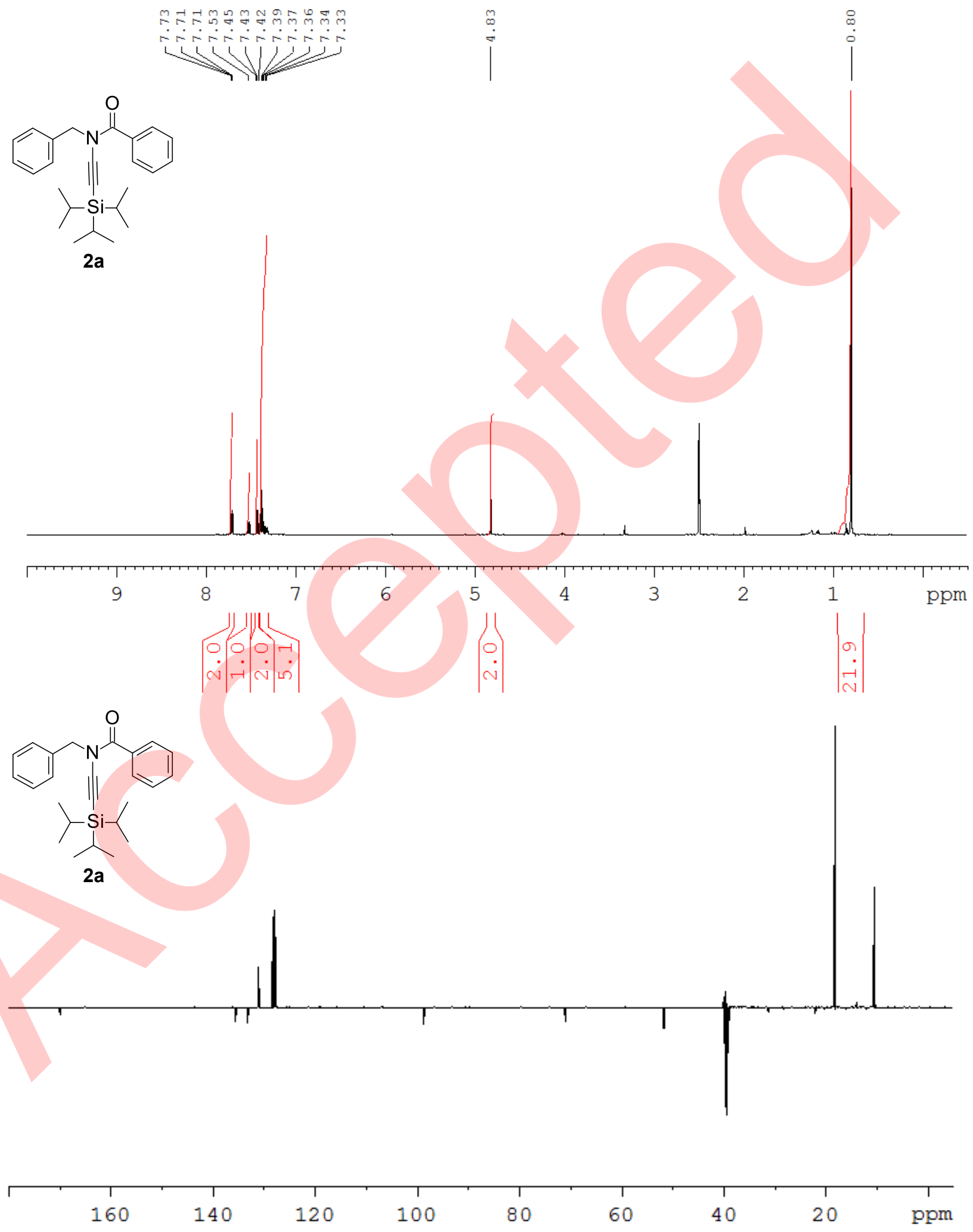



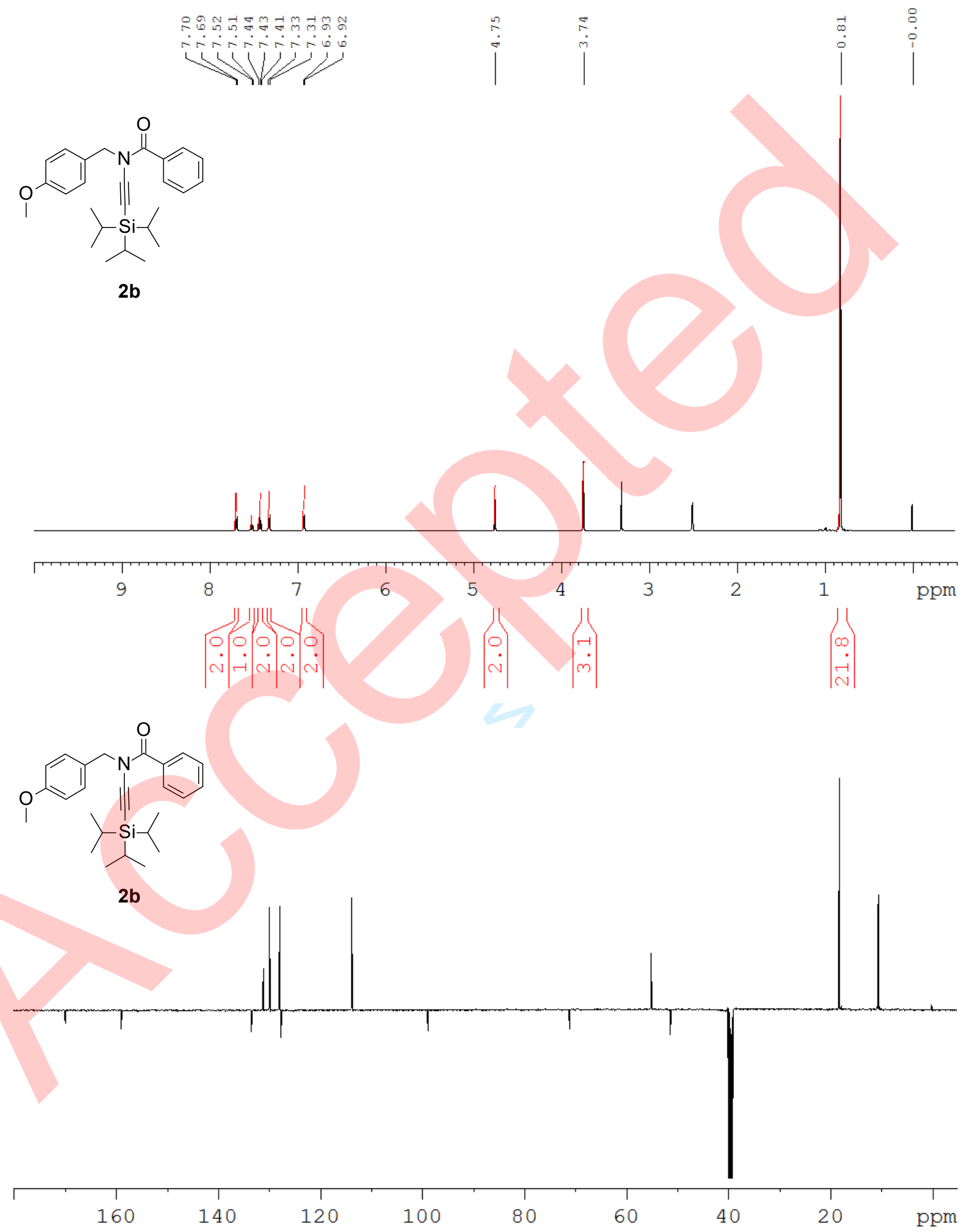

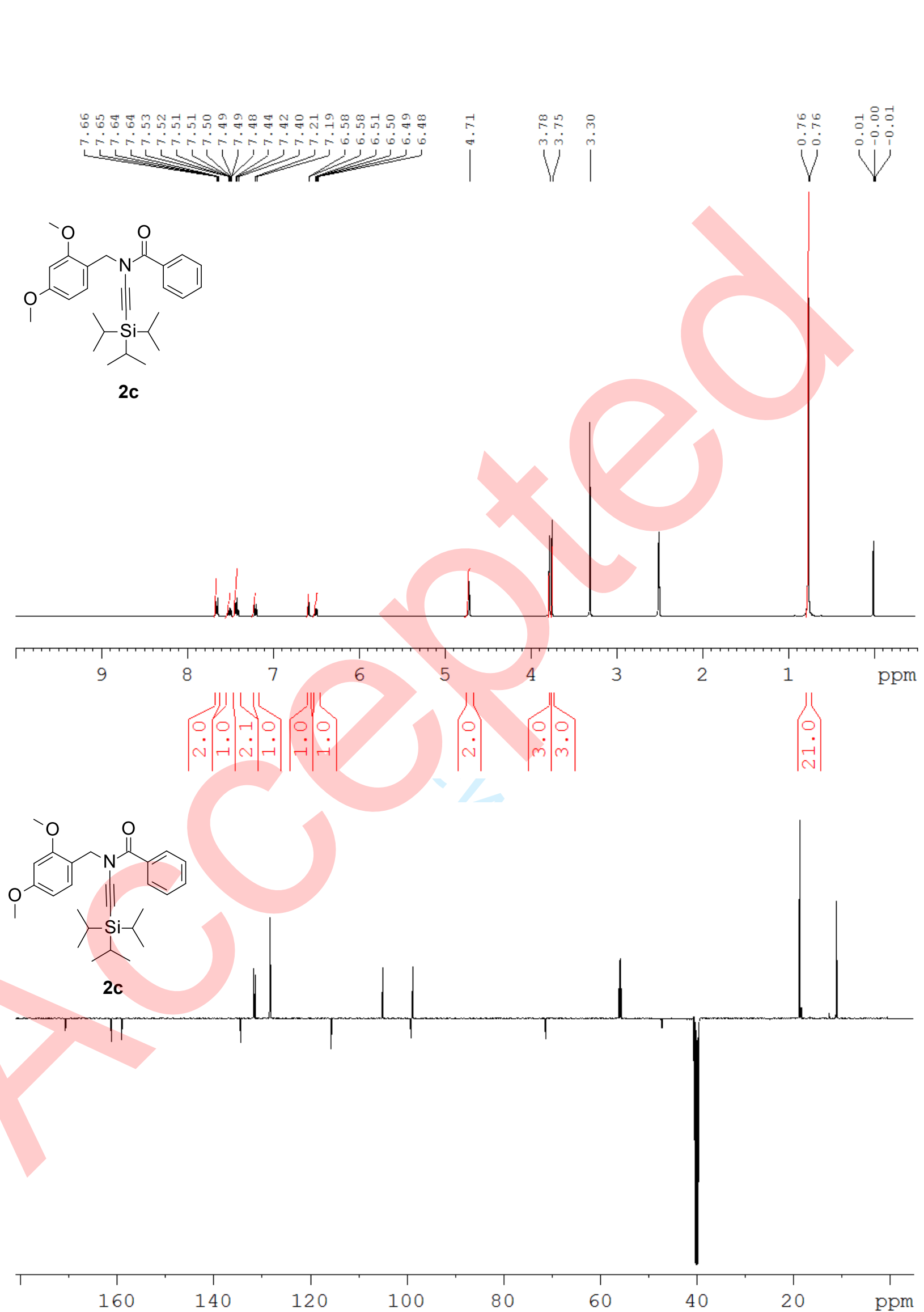


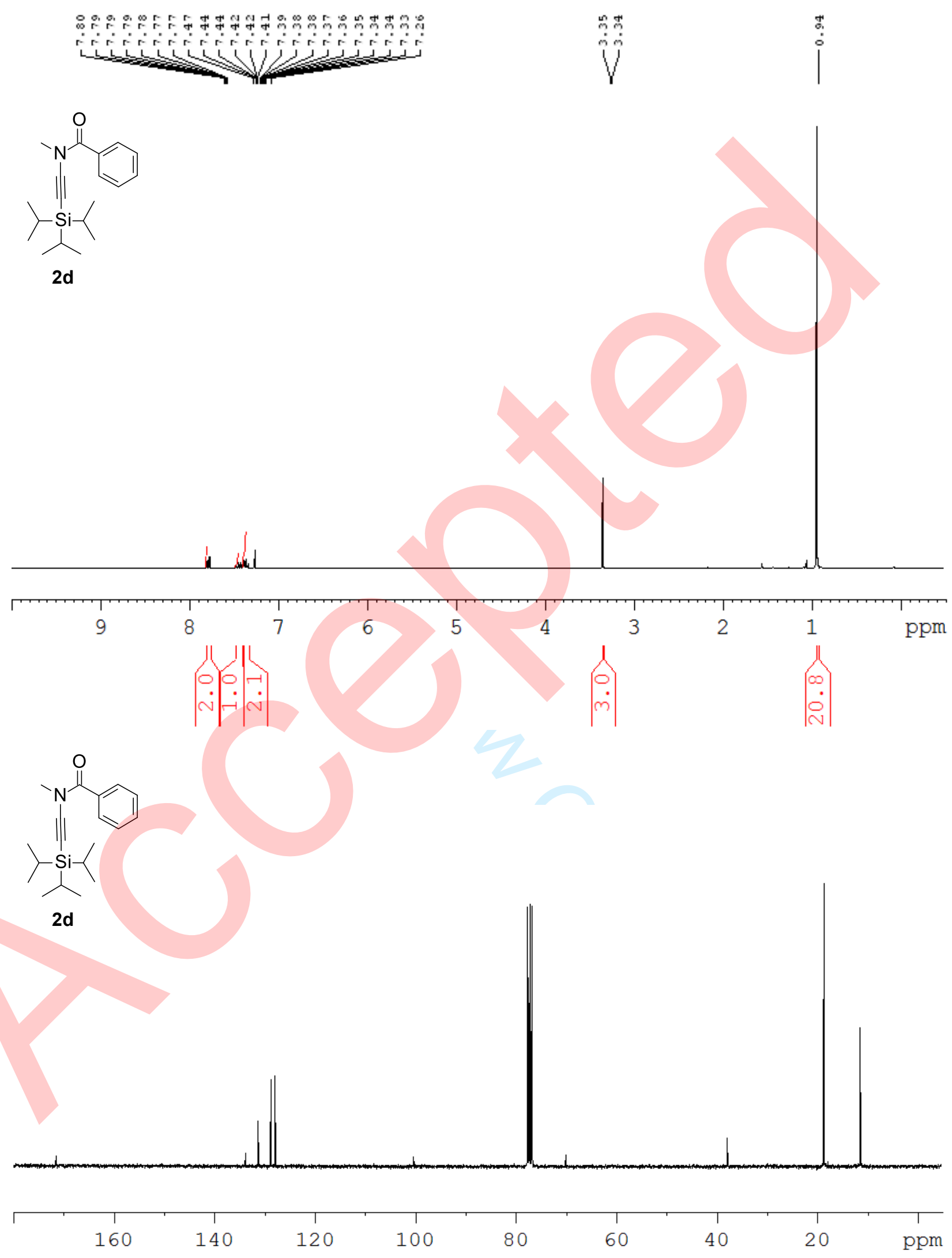




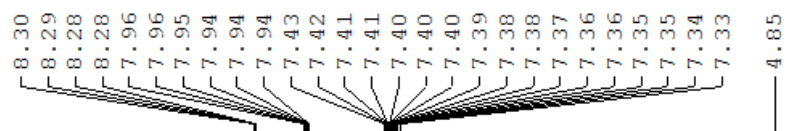<smiles>Cc1ccc(CN(C#Cc2ccccc2)C(=O)c2ccc(C)cc2)cc1</smiles>

$2 \mathrm{e}$
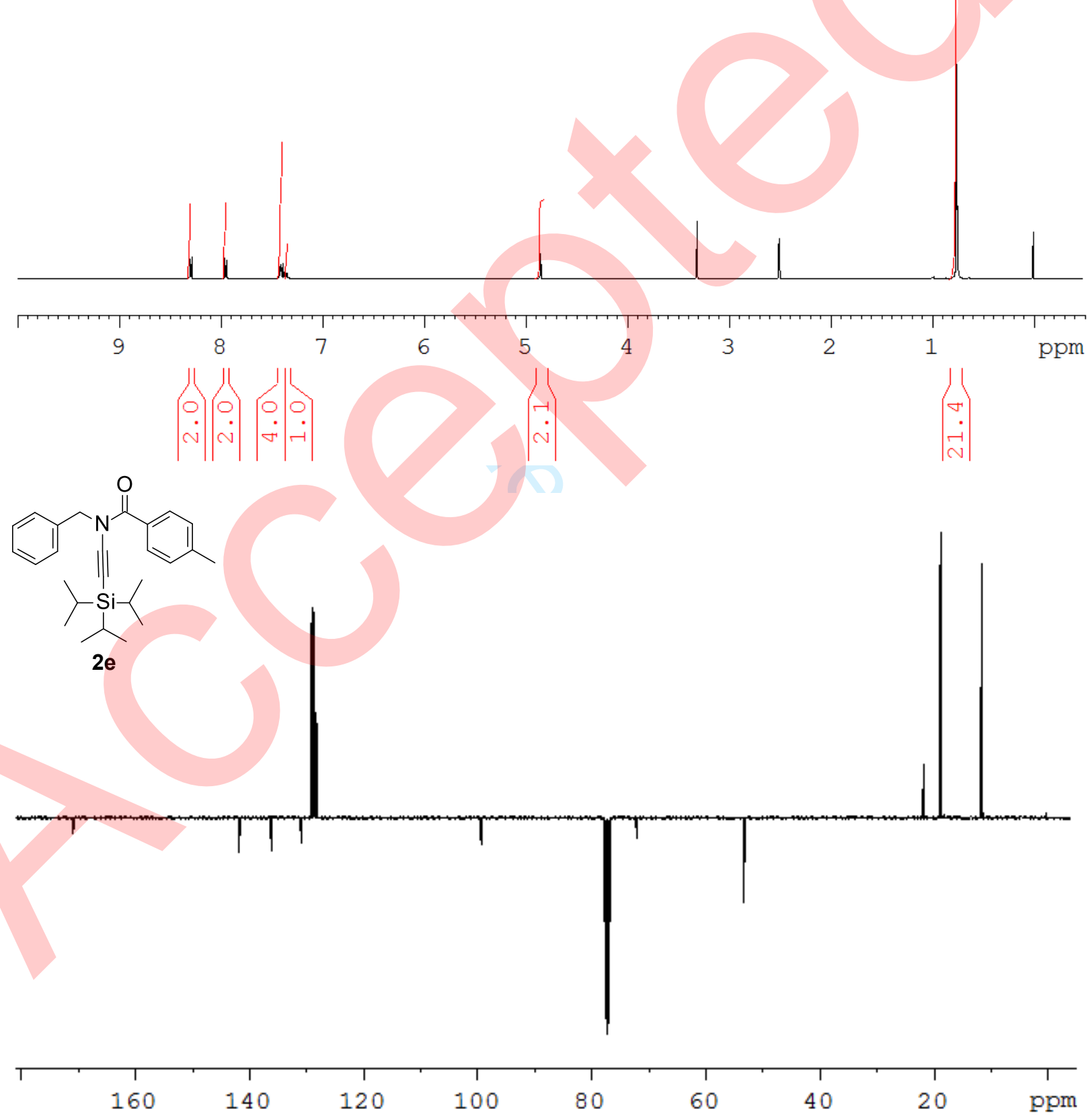


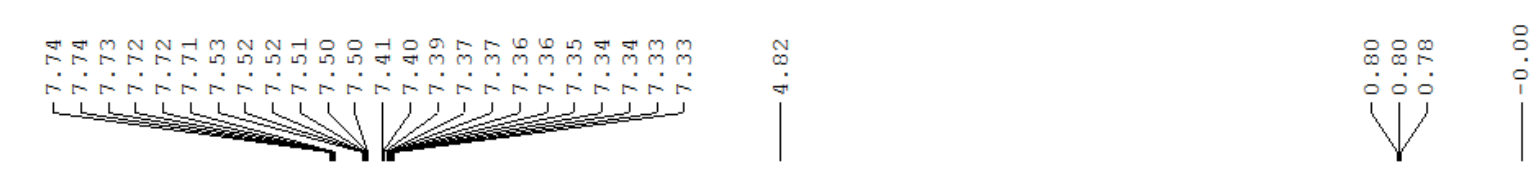<smiles>[Z17]C(C)C(C(C)C)[Si](C#CN(Cc1ccccc1)C(=O)c1ccc(Cl)cc1)(C(C)C)C(C)C</smiles>
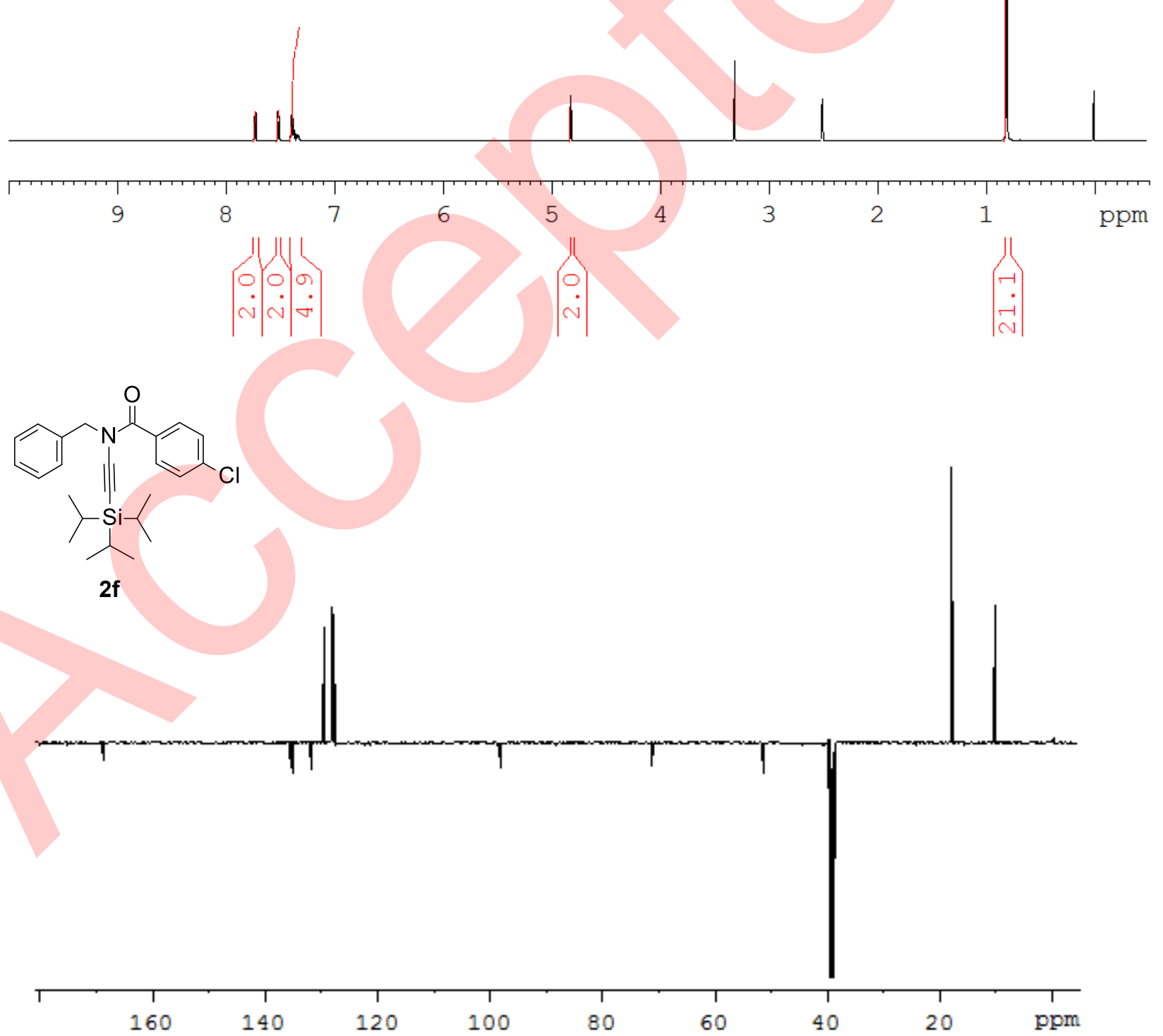


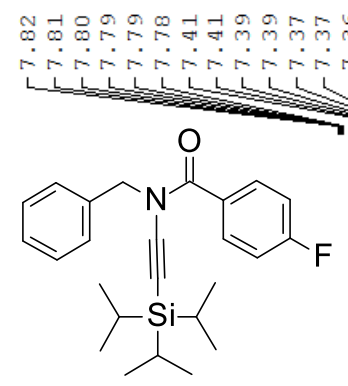

$2 \mathrm{~g}$
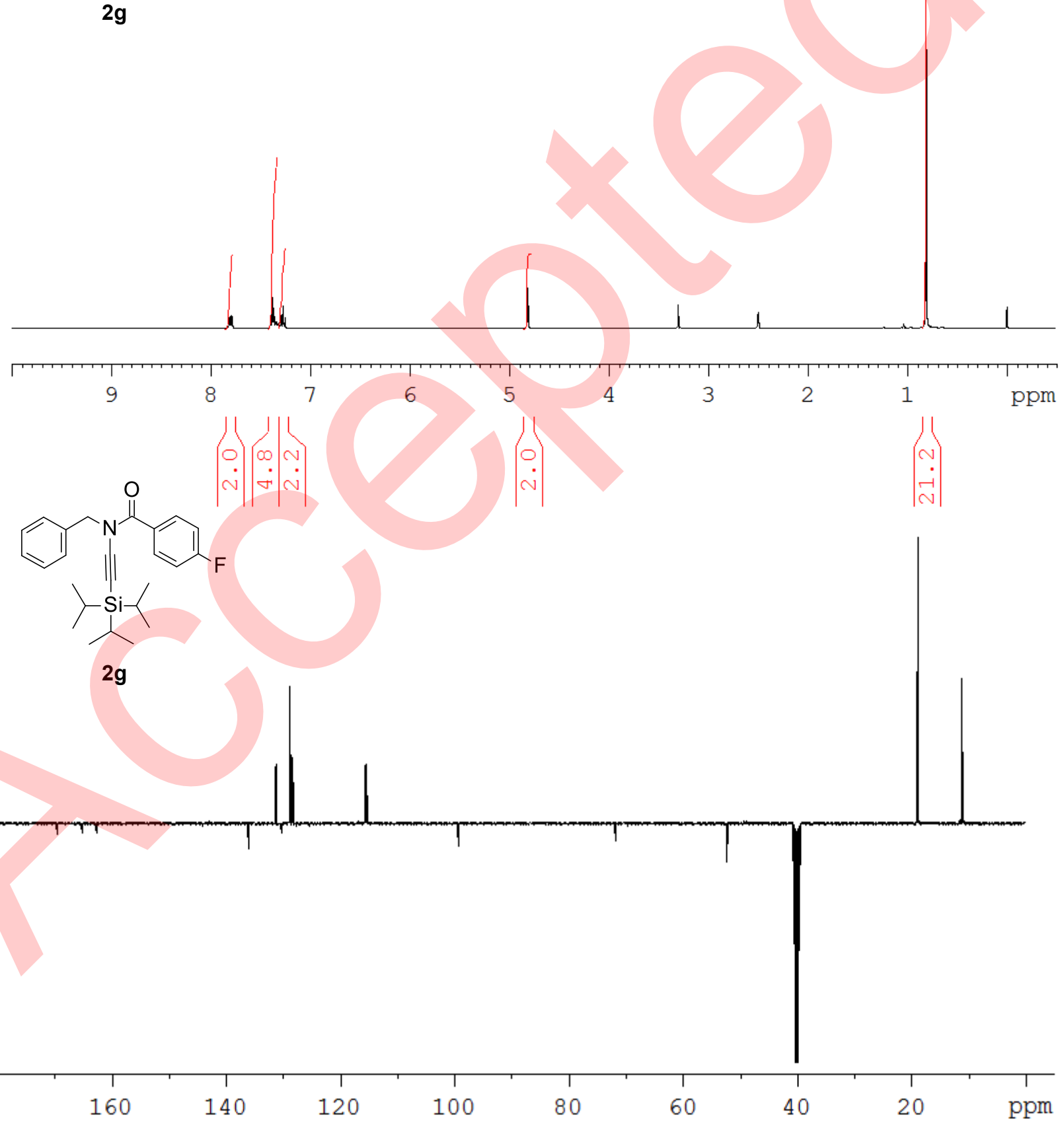


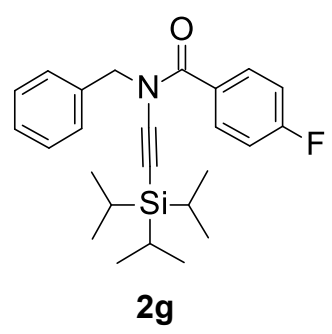

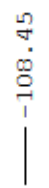

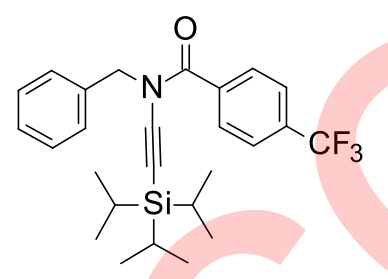

2h

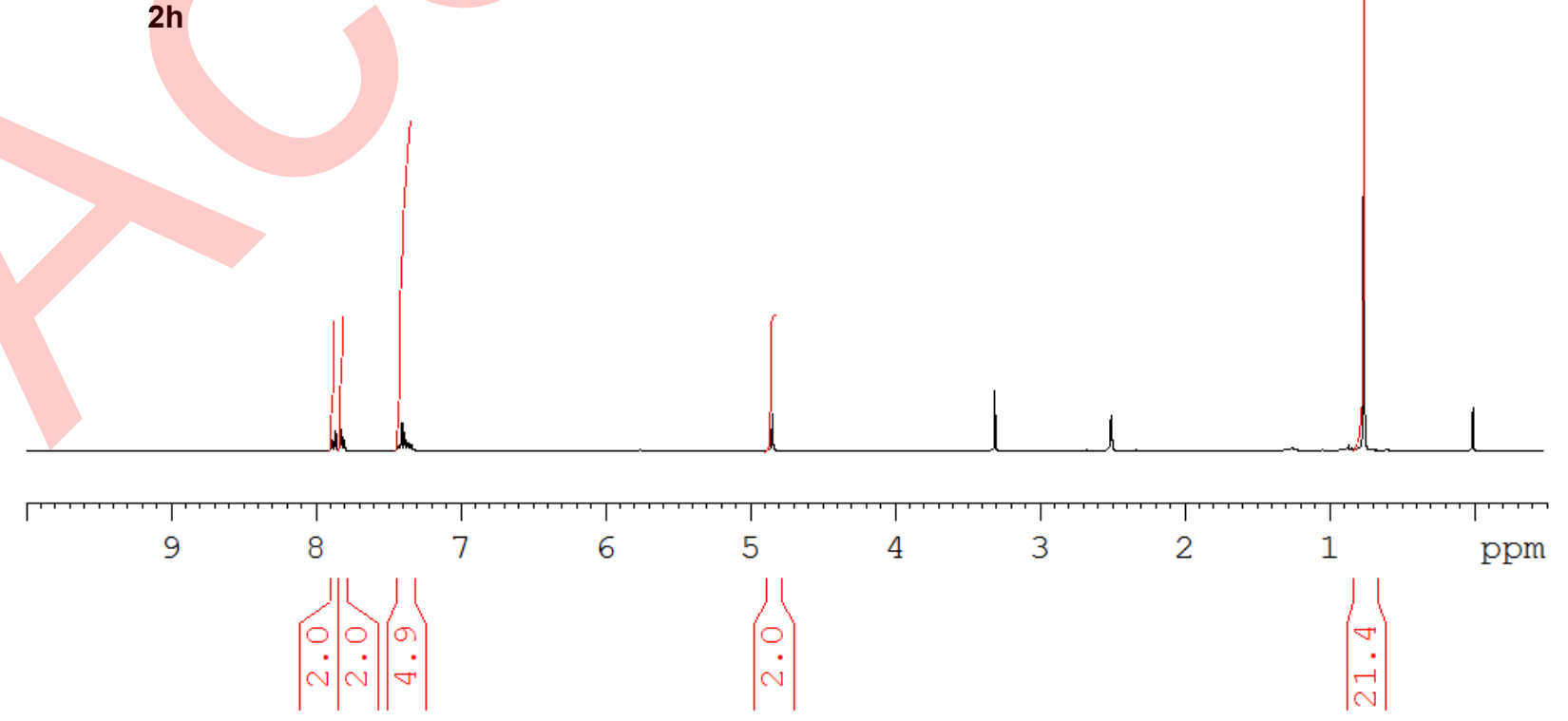




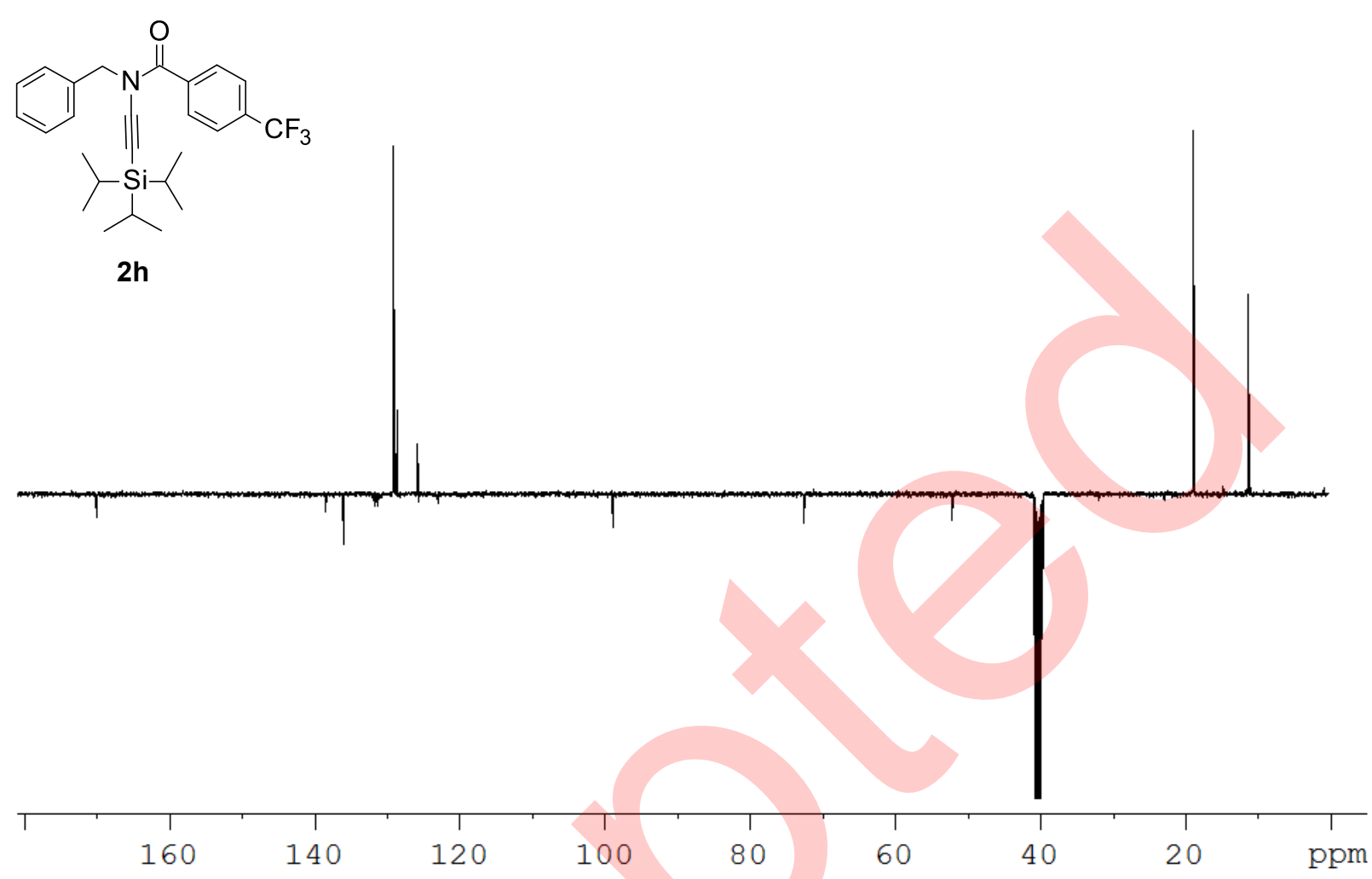




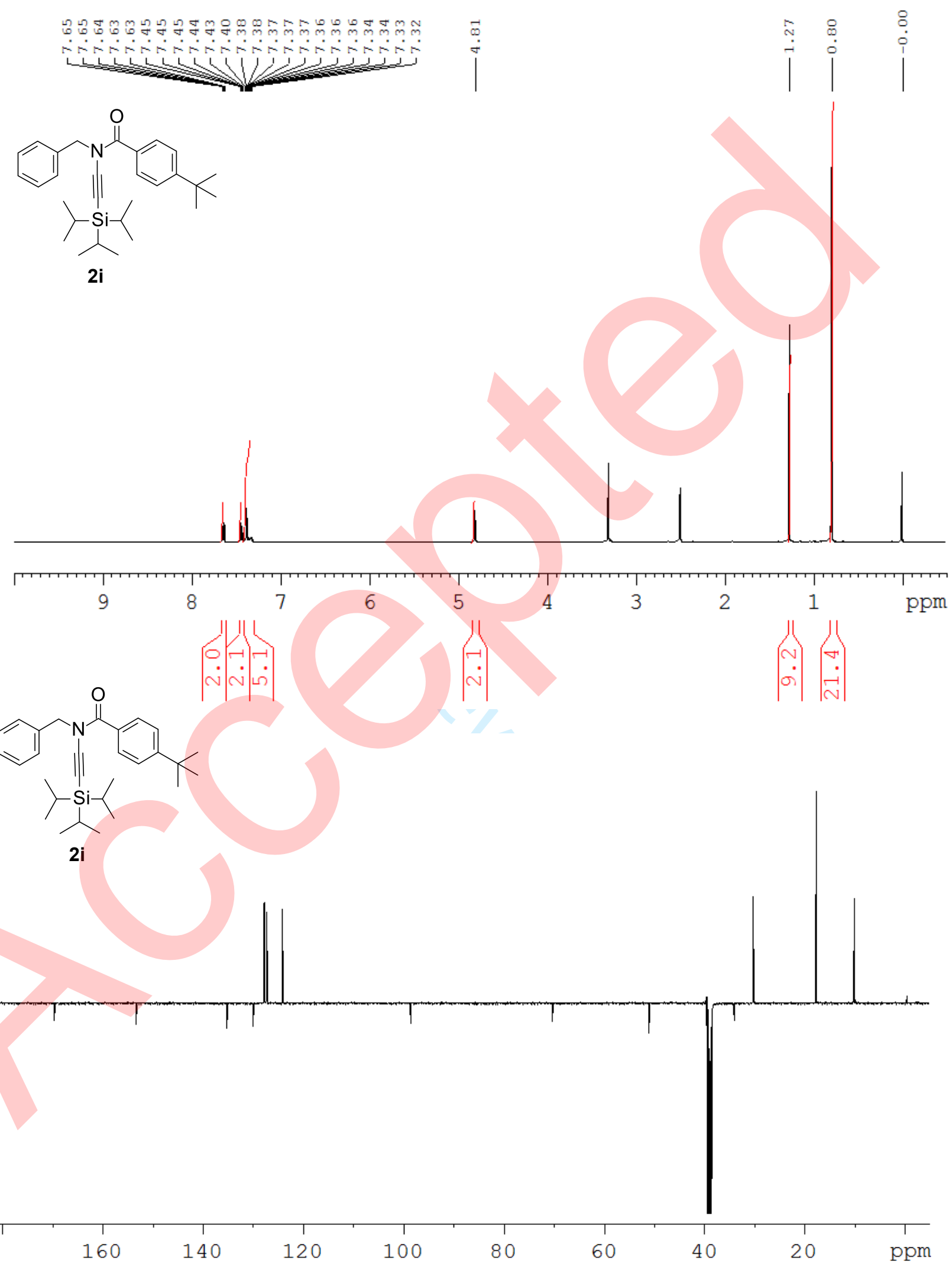



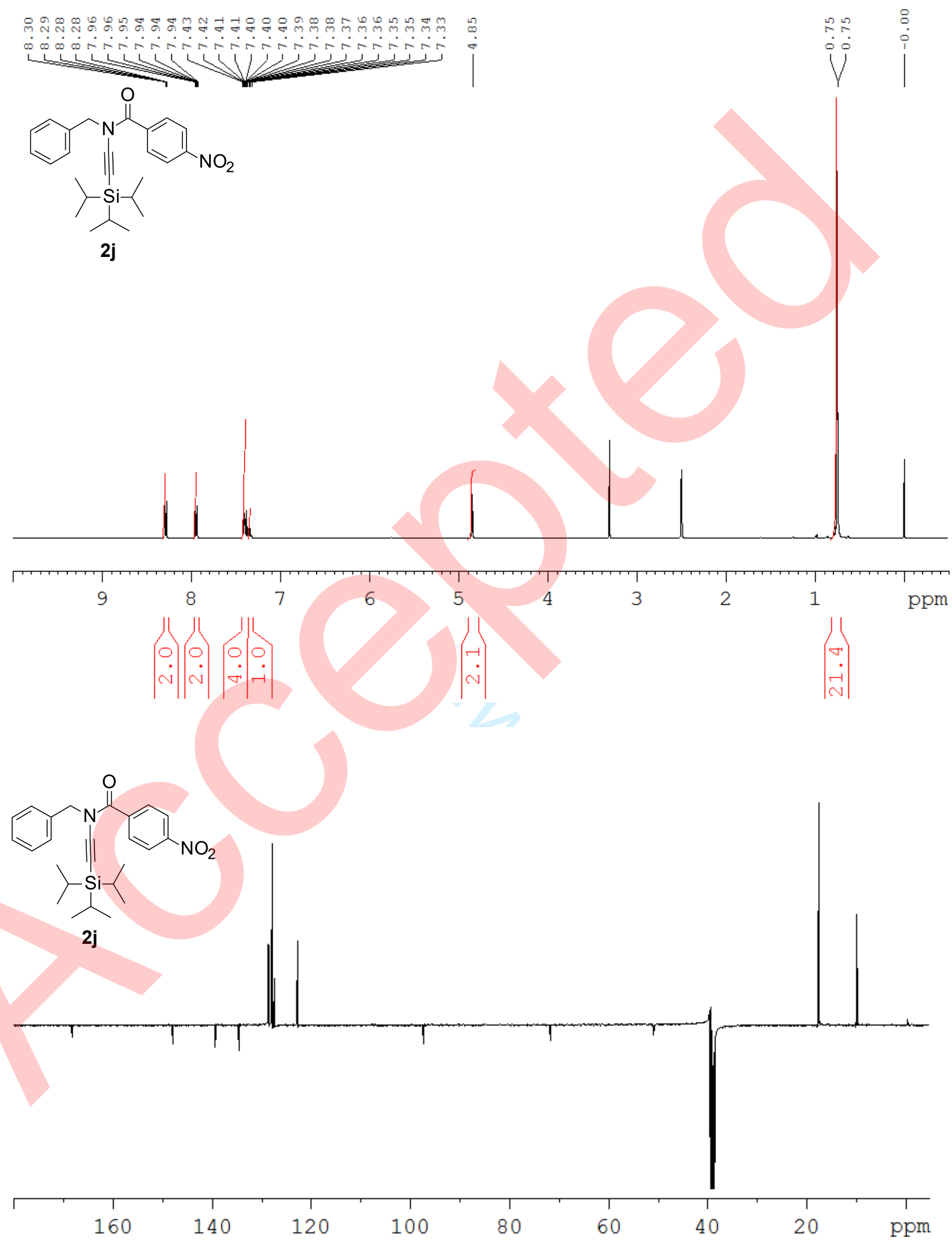


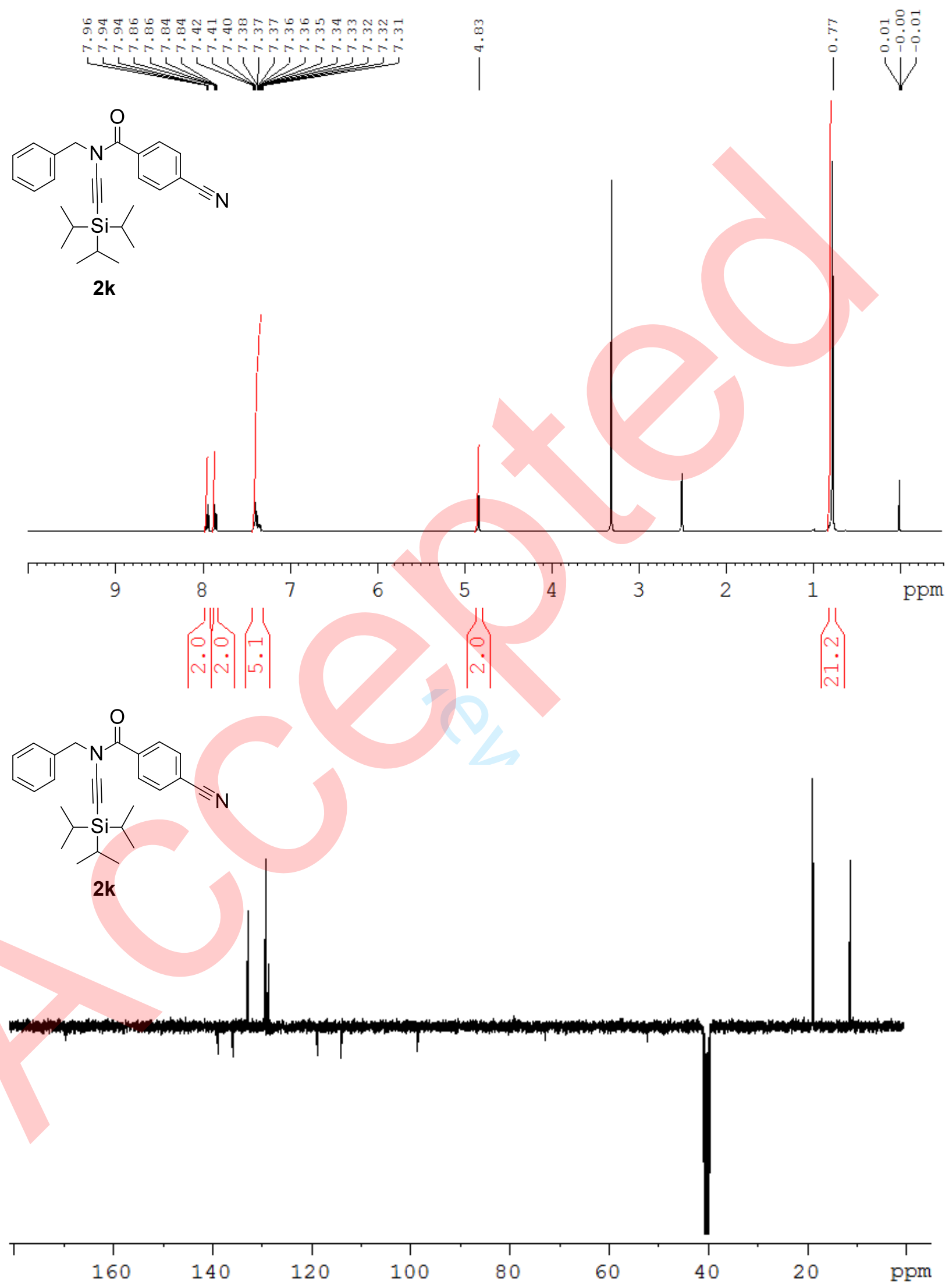




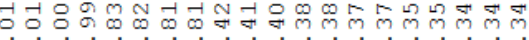

onsing

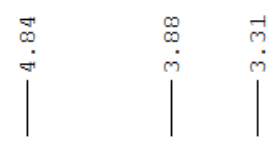

눈<smiles>COC(=O)c1ccc(C(=O)N(C#CN(Cc2ccccc2)C(C)C)C(C)C)cc1</smiles>

2I
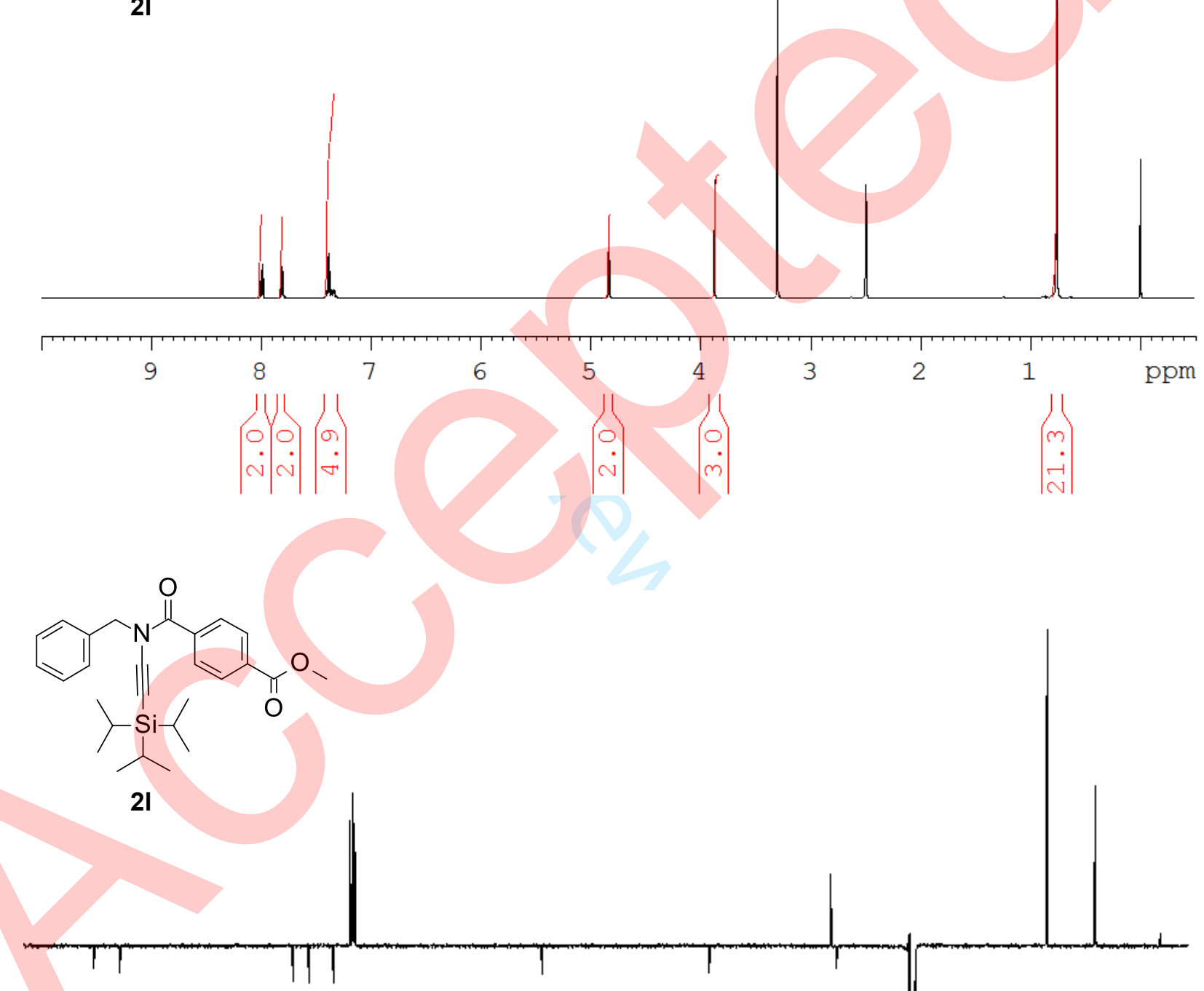

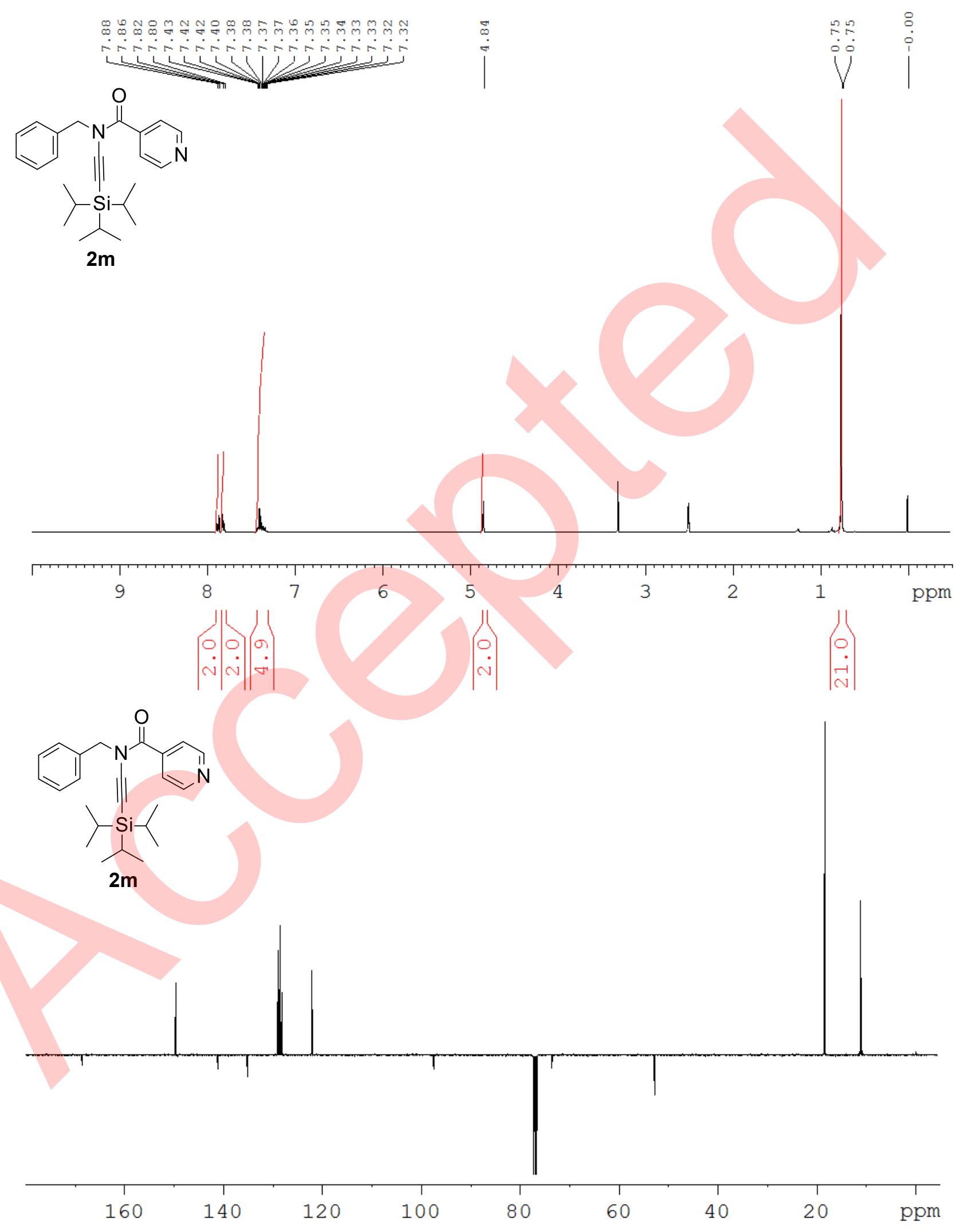


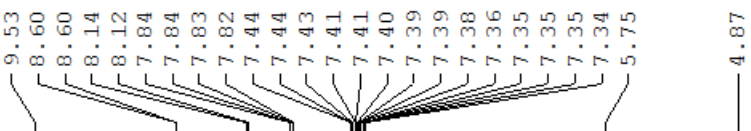<smiles>CC(C)[Si](C#CN(Cc1ccccc1)C(=O)c1ccc2c(c1)SCN2)(C(C)C)C(C)C</smiles>

2n

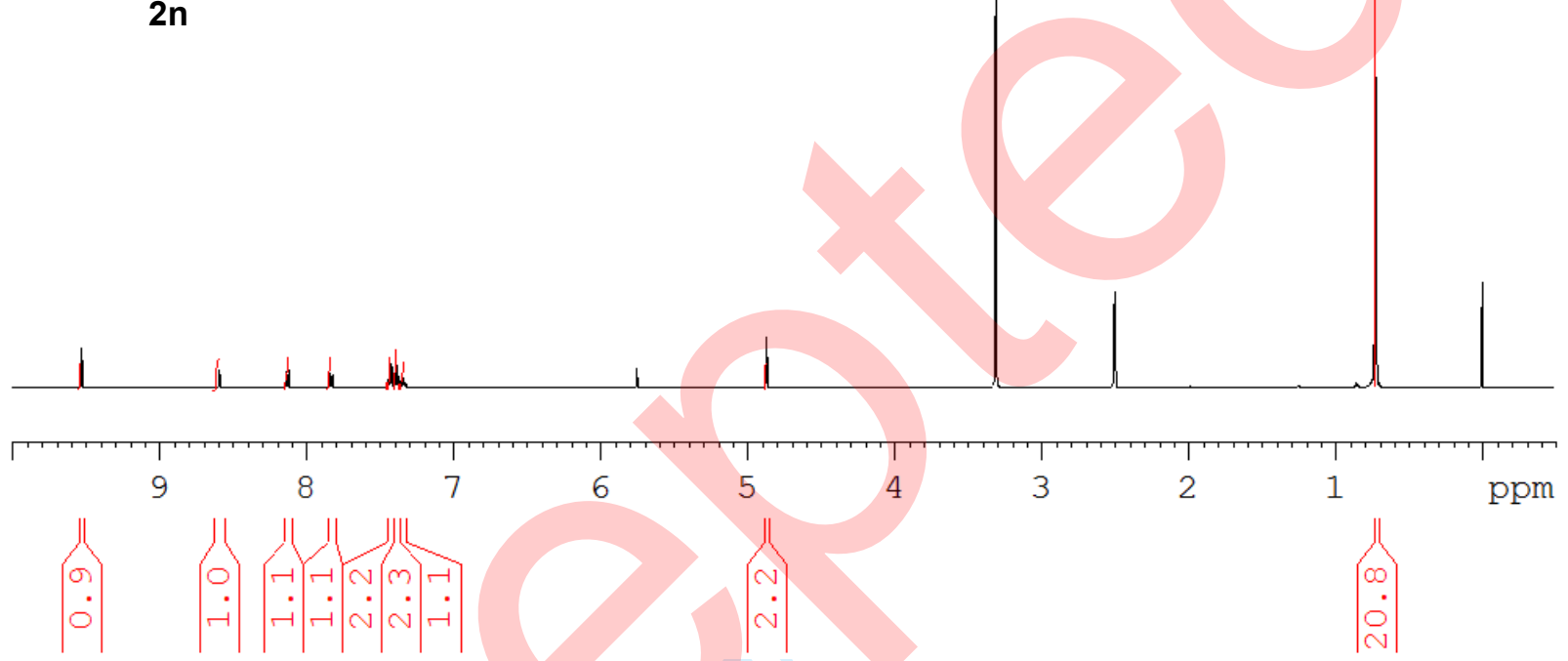<smiles>CC(C)[Si](C#CN(Cc1ccccc1)C(=O)c1ccc2ncsc2c1)(C(C)C)C(C)C</smiles>

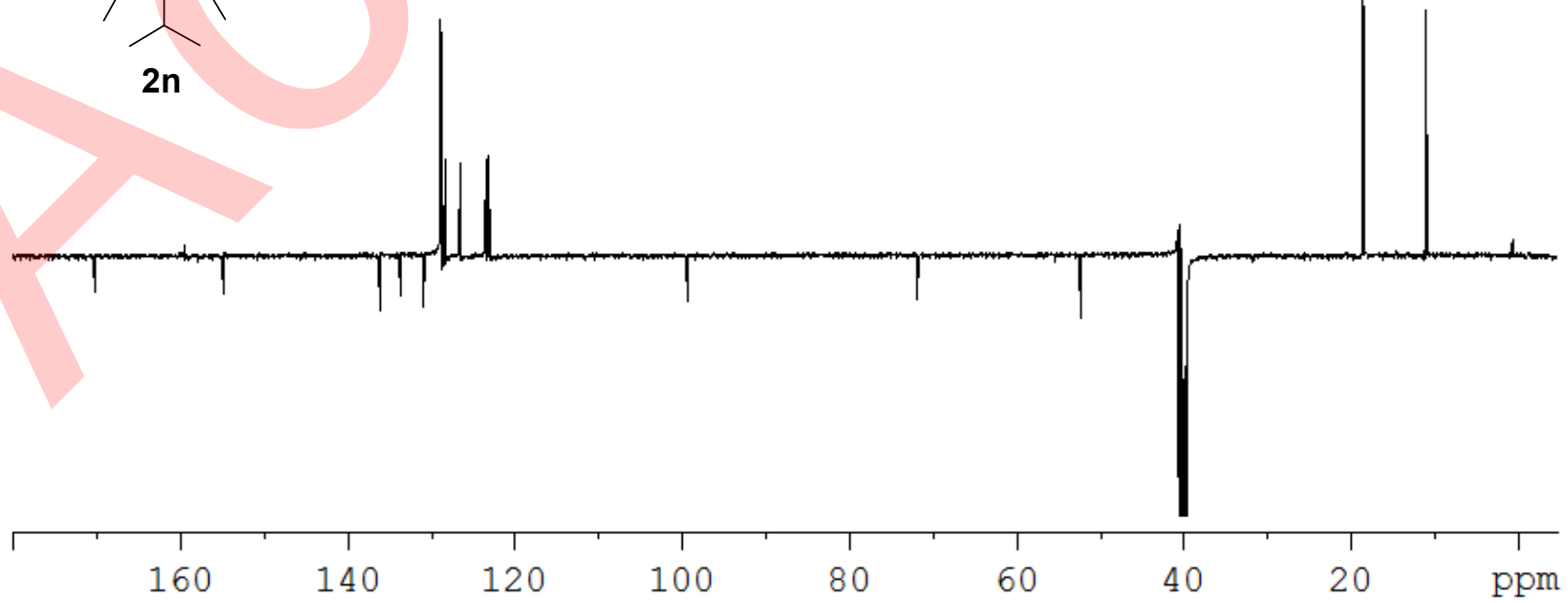




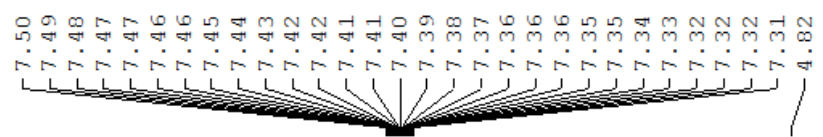<smiles>CC(C)[Si](C#CN(Cc1ccccc1)C(=O)c1cc(F)cc(F)c1)(C(C)C)C(C)C</smiles>

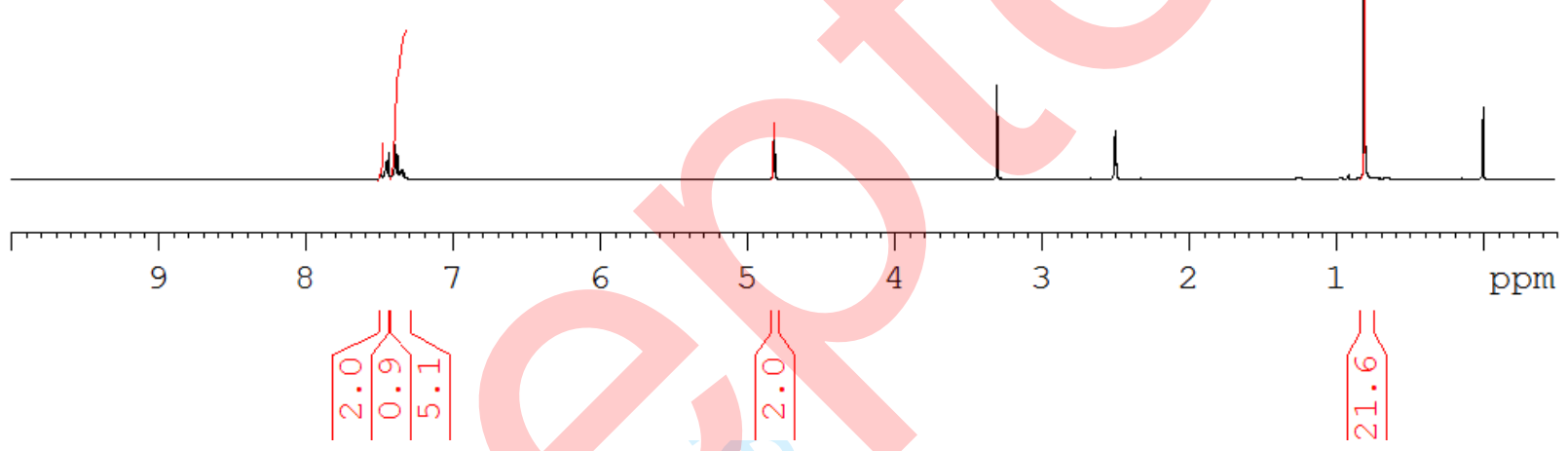<smiles>CC(C)[Si](C#CN(Cc1ccccc1)C(=O)c1cc(F)cc(F)c1)(C(C)C)C(C)C</smiles>
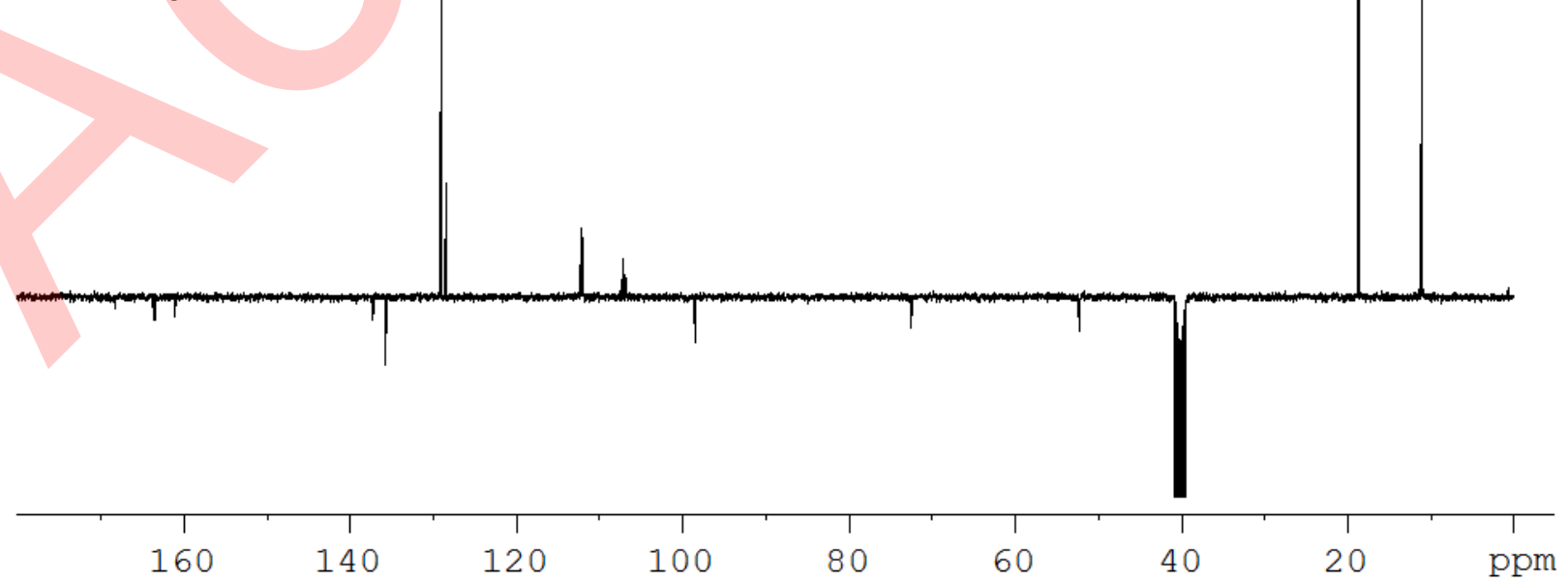


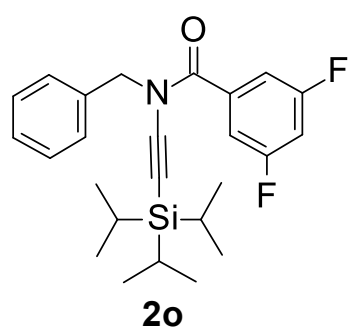

$$
\stackrel{\infty}{\infty}
$$
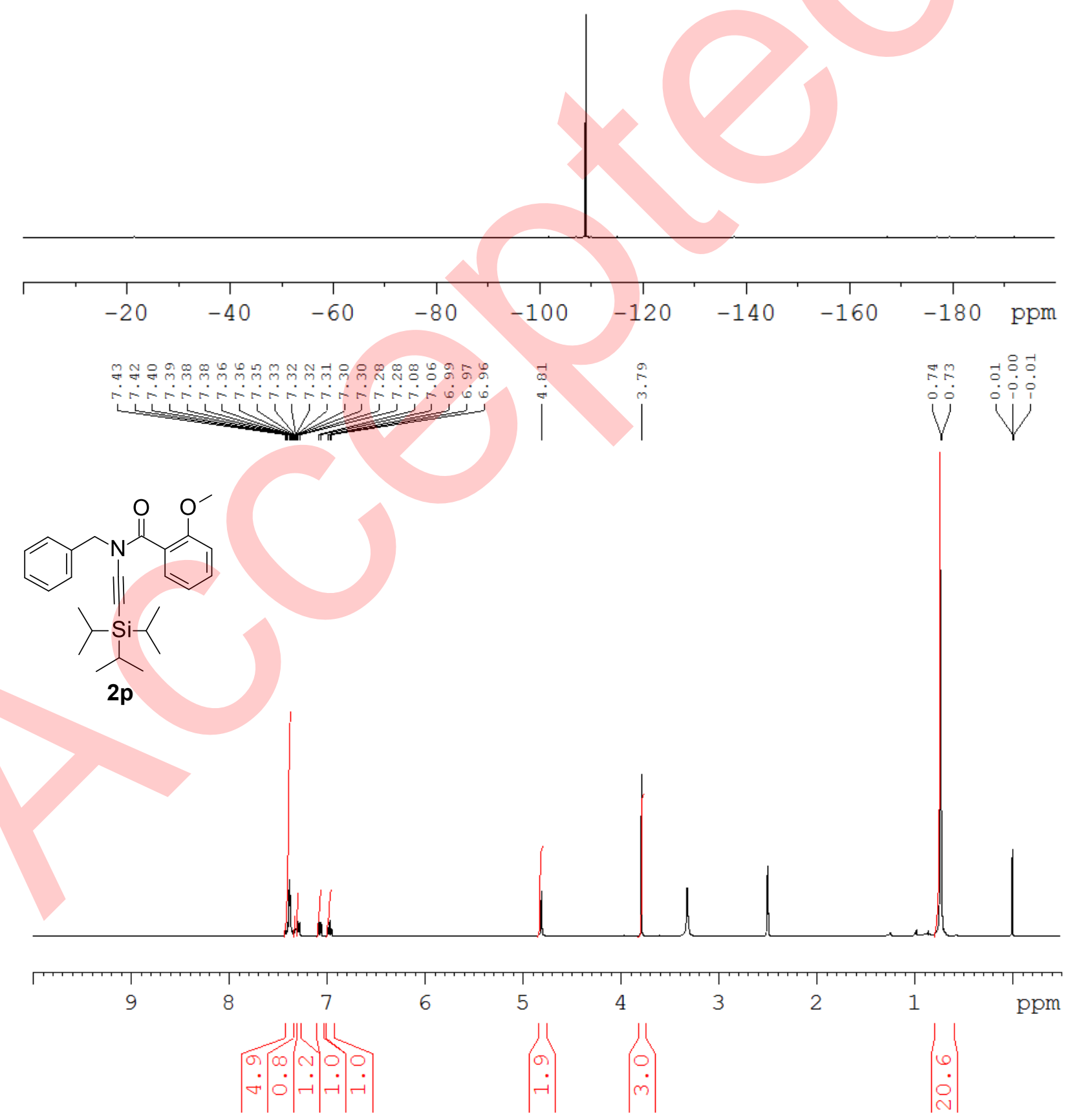

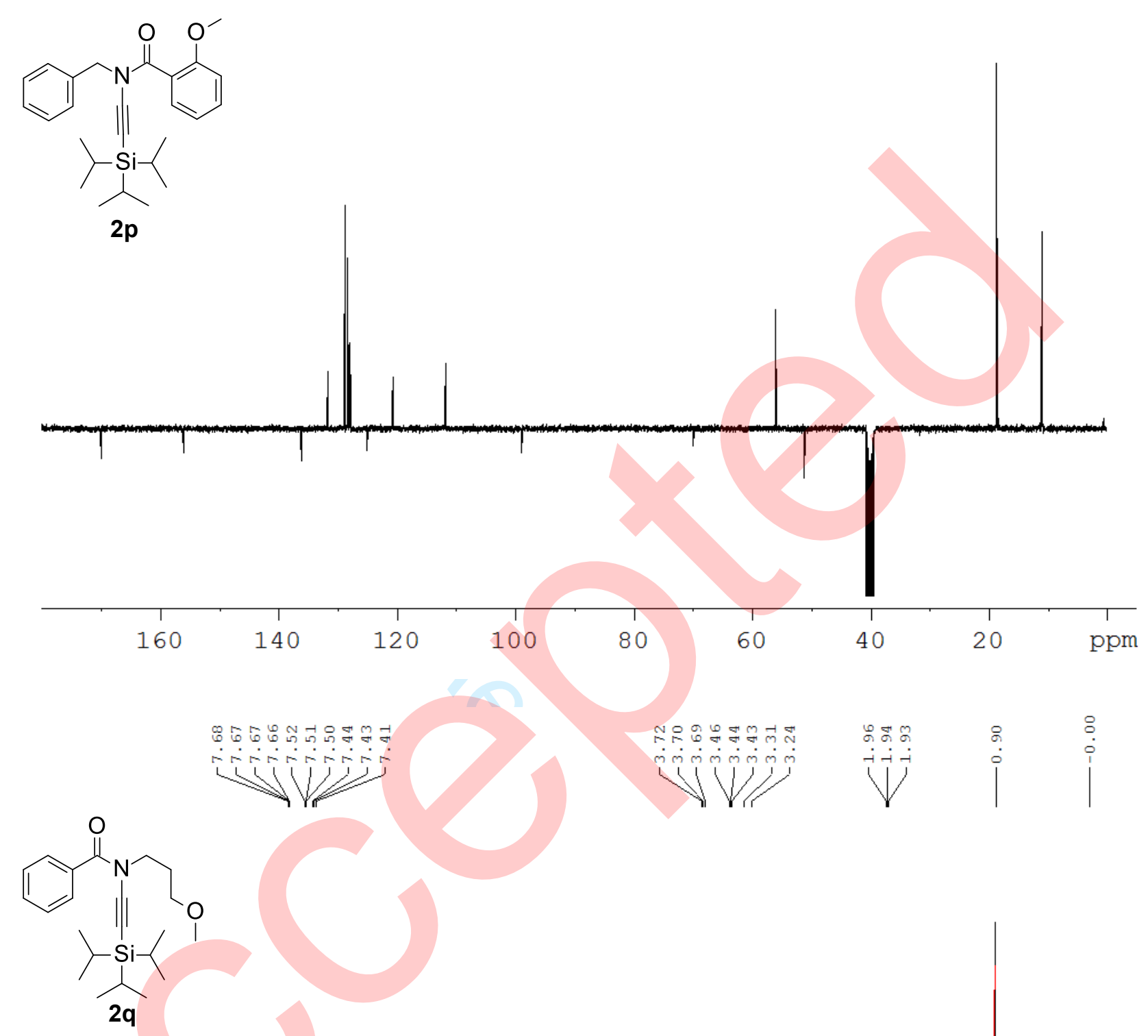

41

42

43 

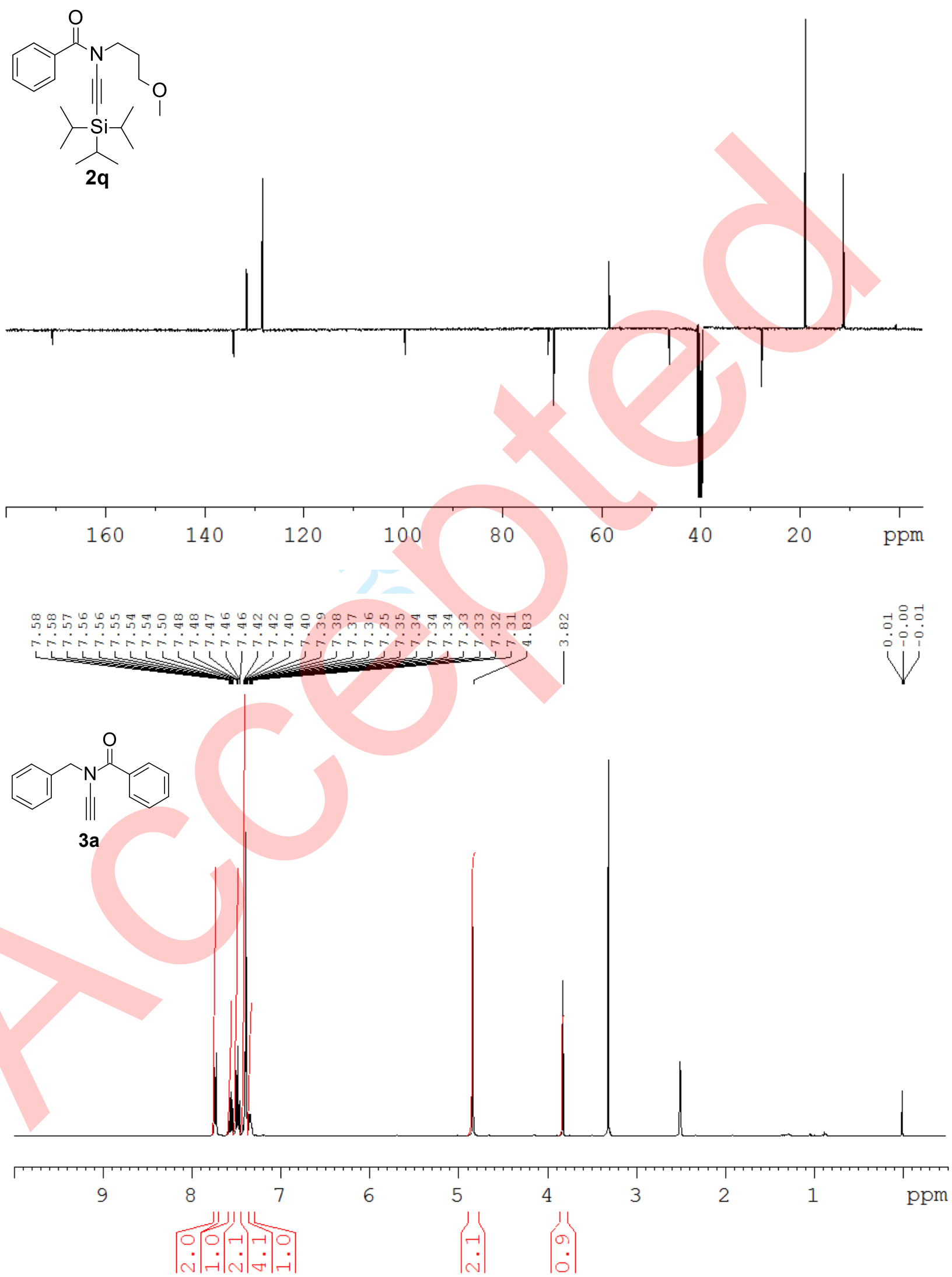

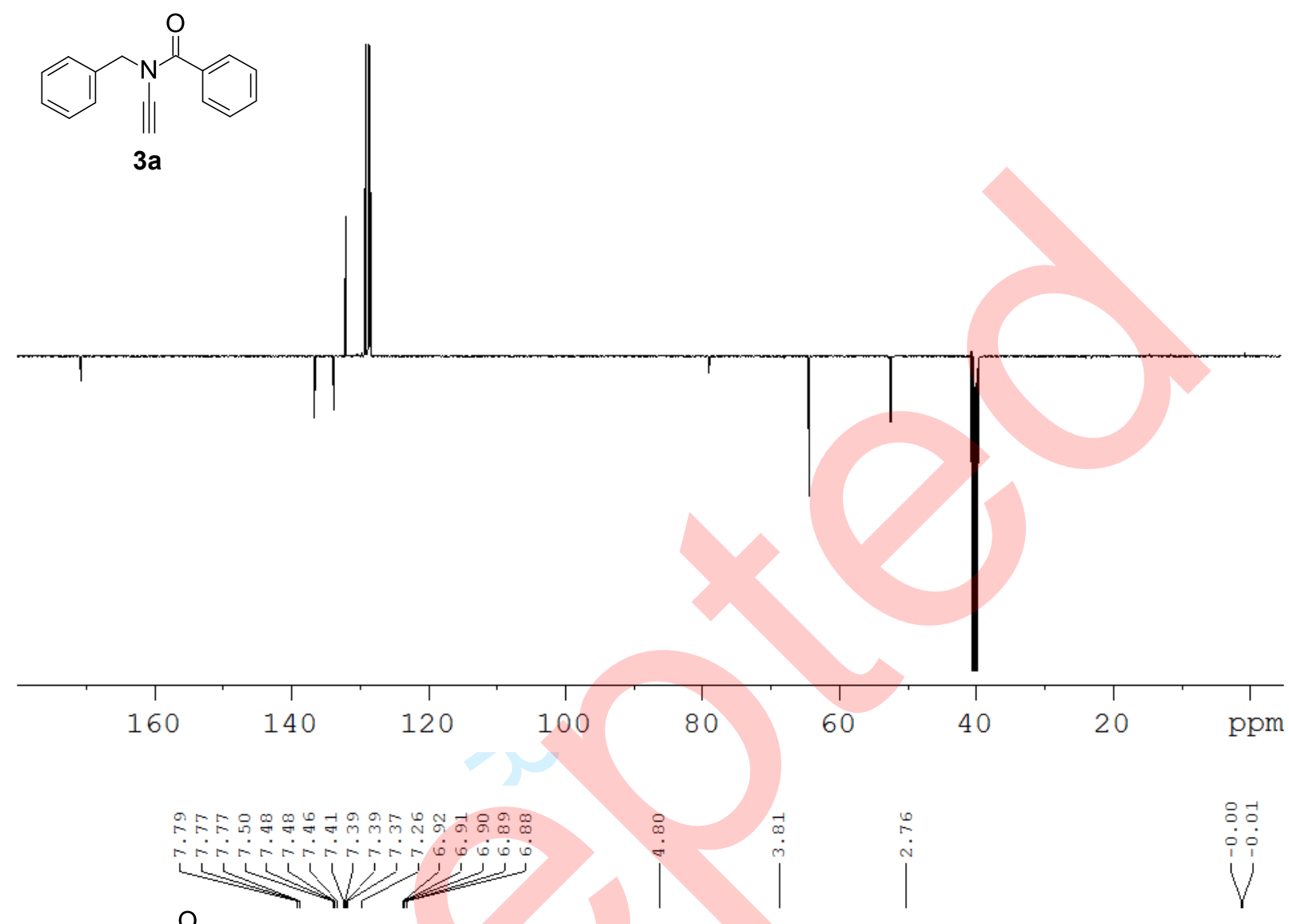<smiles>C#CN(Cc1ccc(OC)cc1)C(=O)c1ccccc1</smiles>

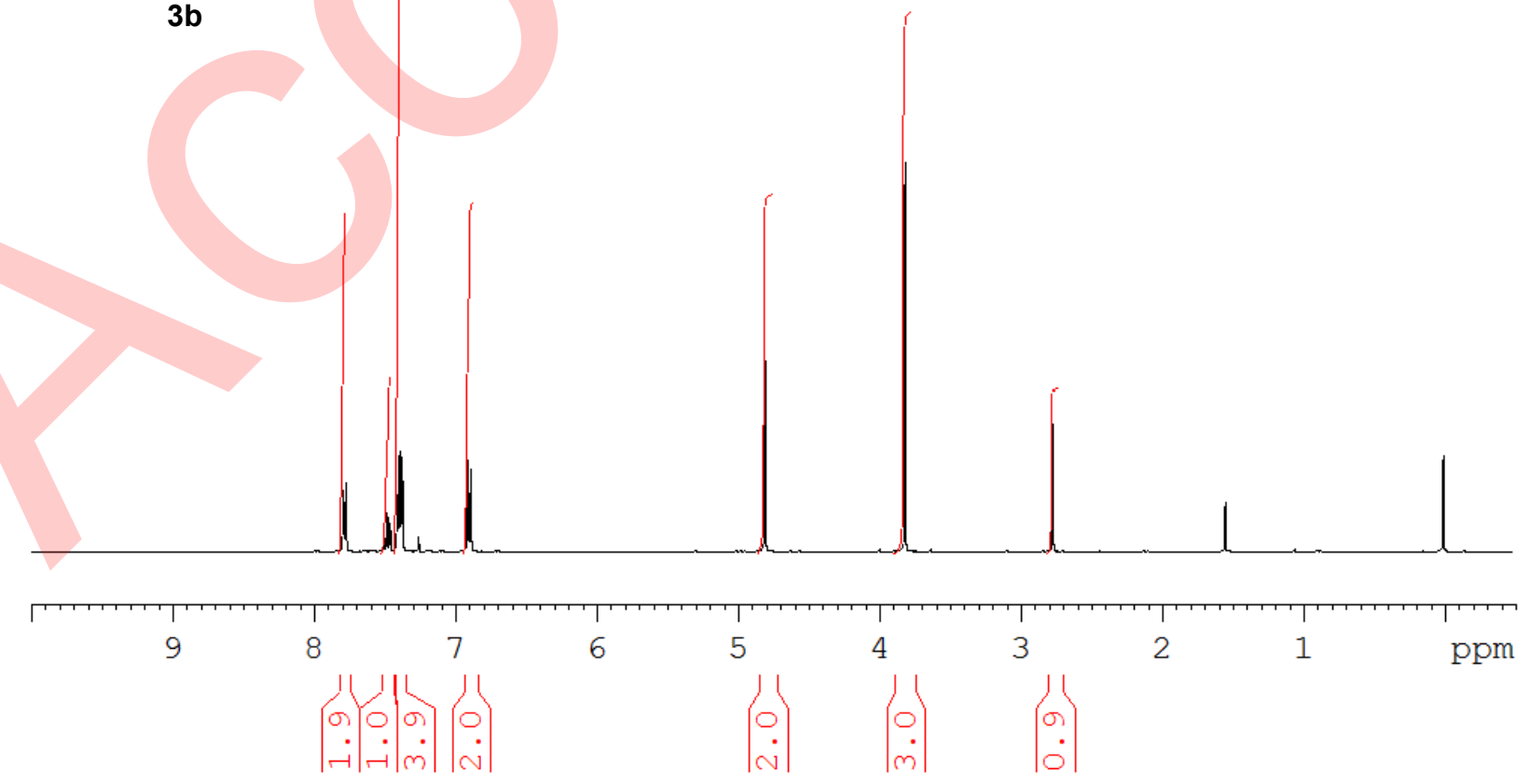


<smiles>C#CN(Cc1ccc(OC)cc1)C(=O)c1ccccc1</smiles>

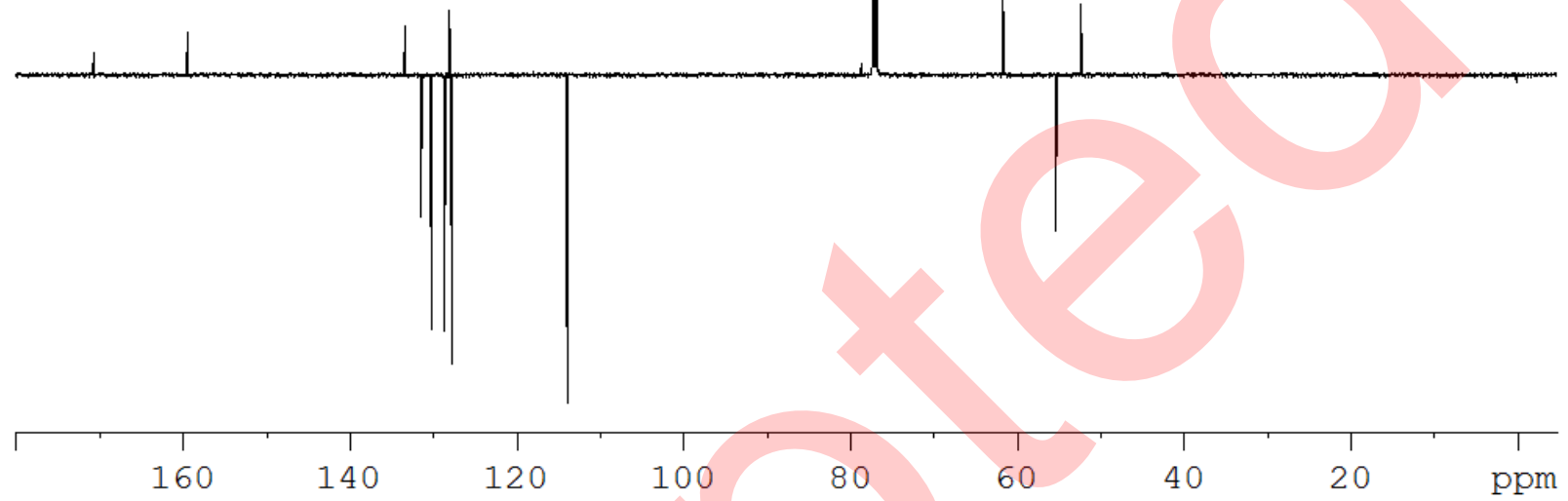

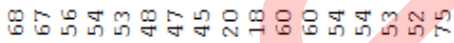

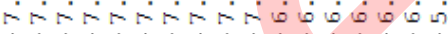

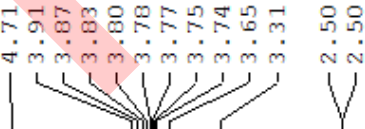

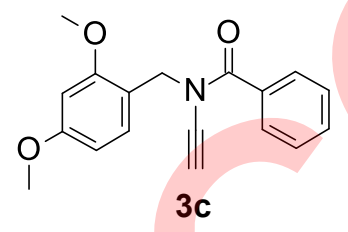
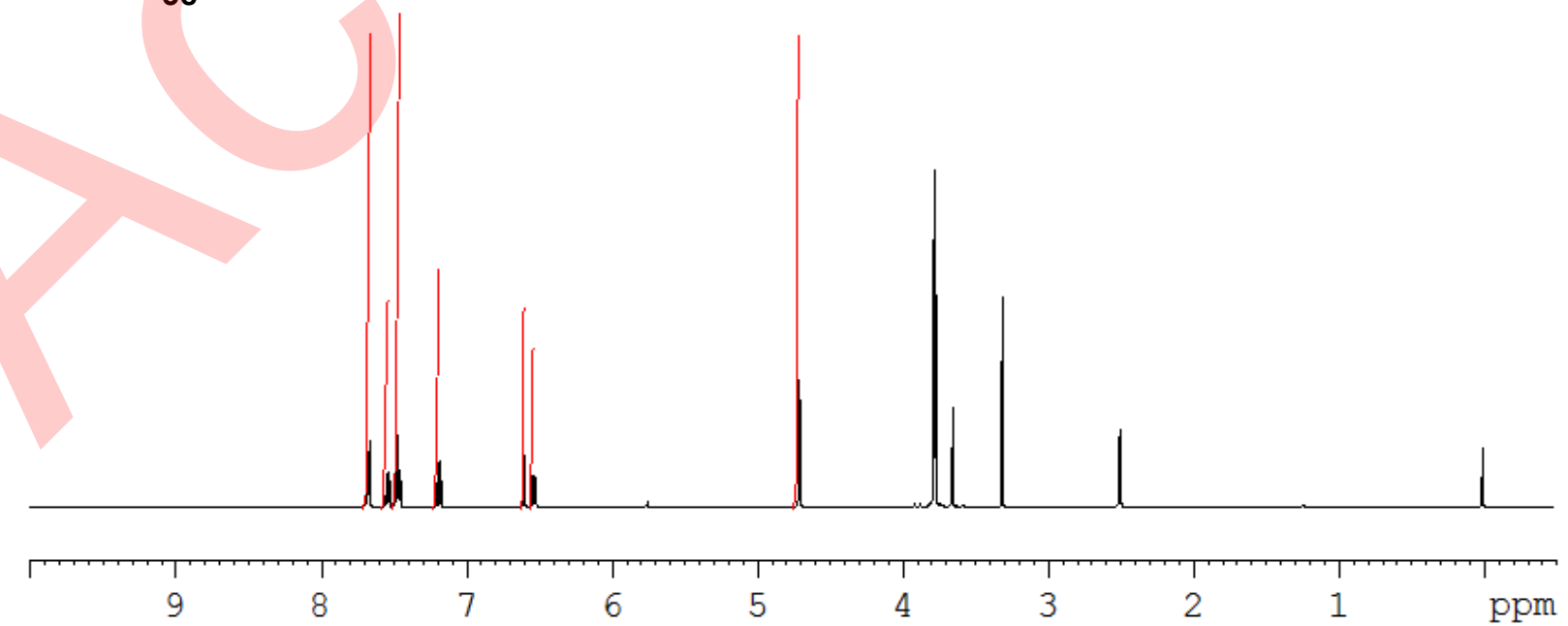

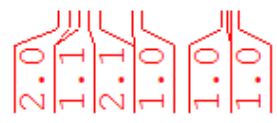

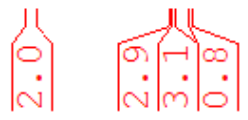



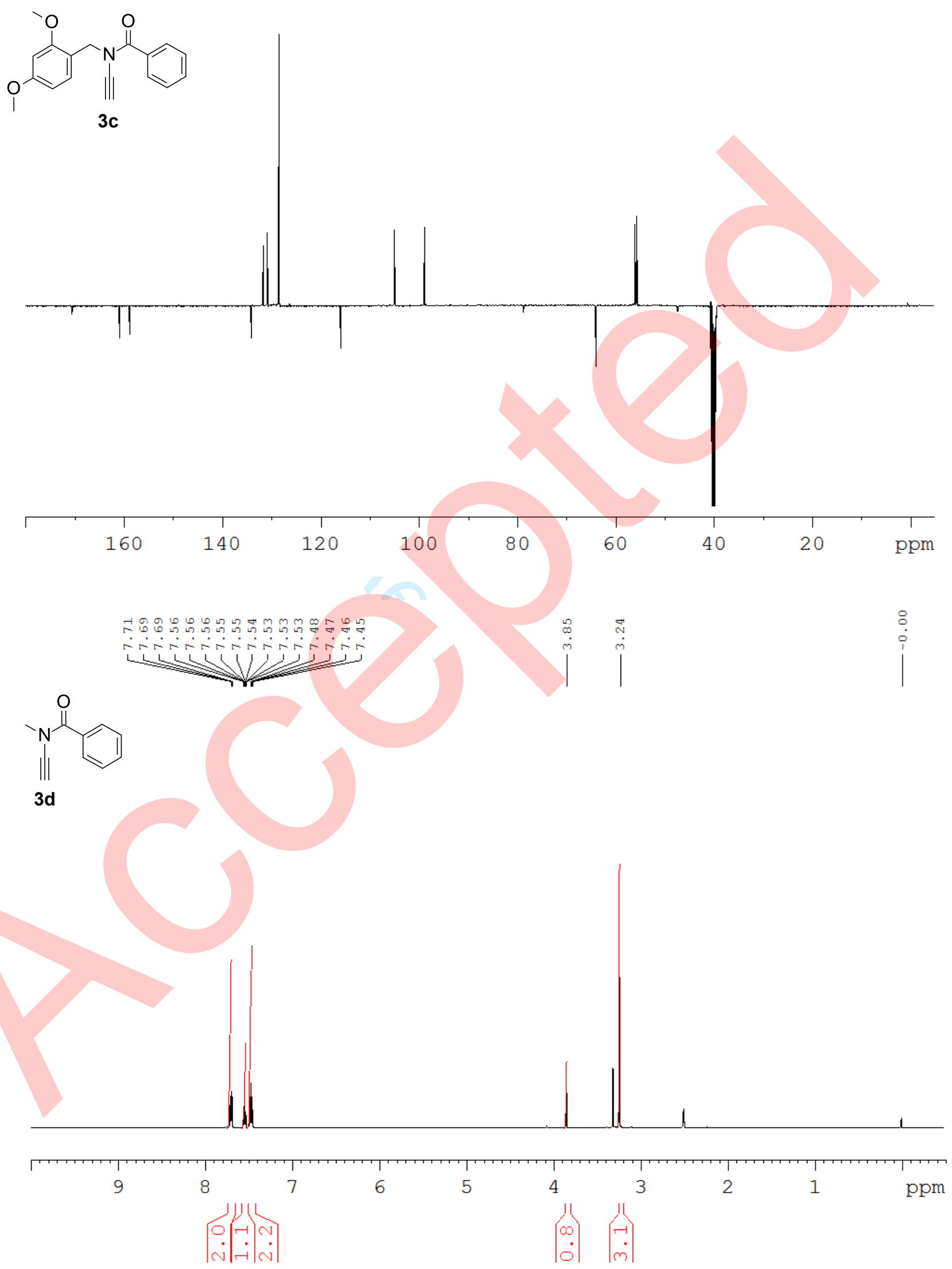

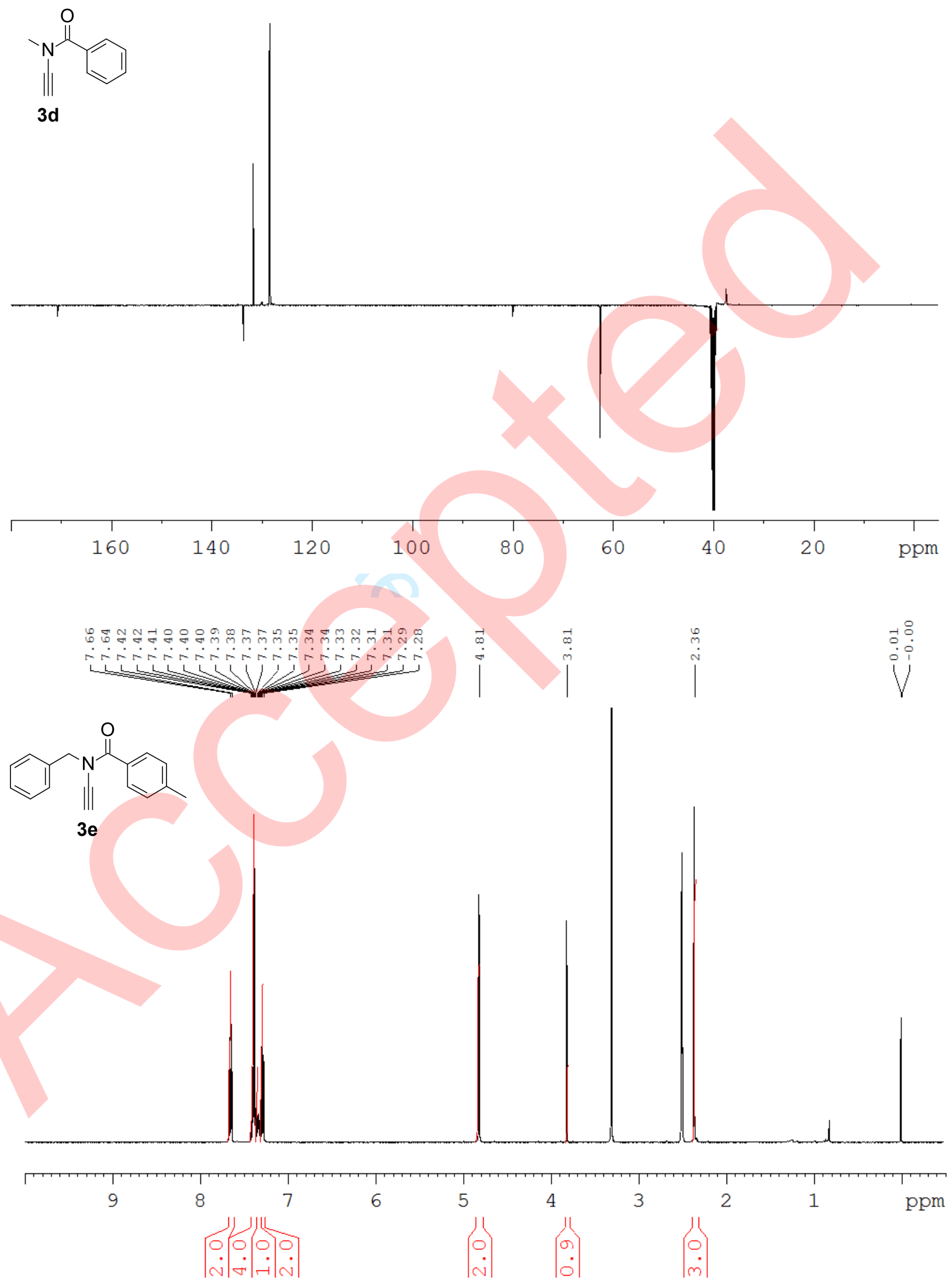

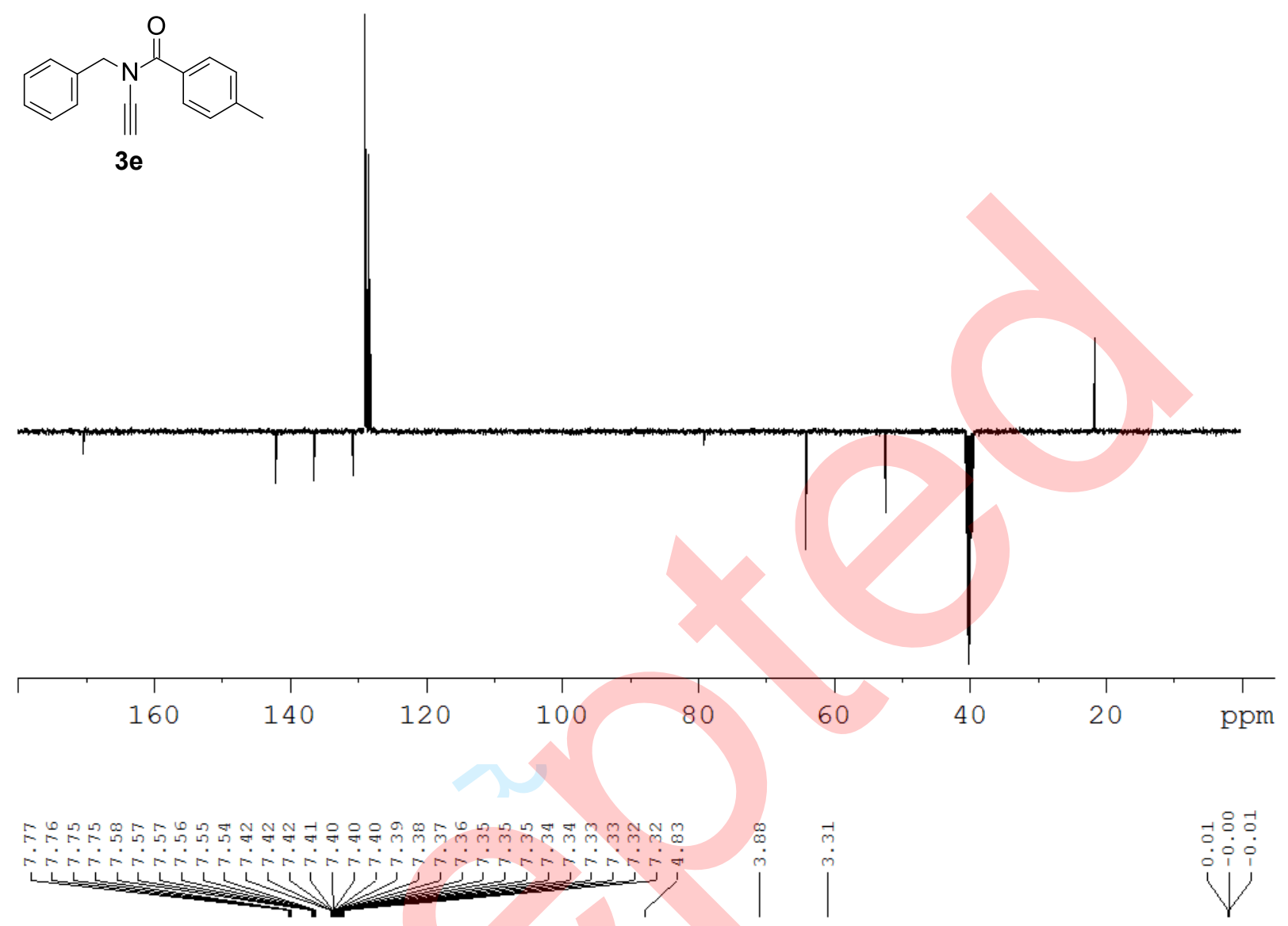<smiles>[13CH3]CN(Cc1ccccc1)C(=O)c1ccc(Cl)cc1</smiles>

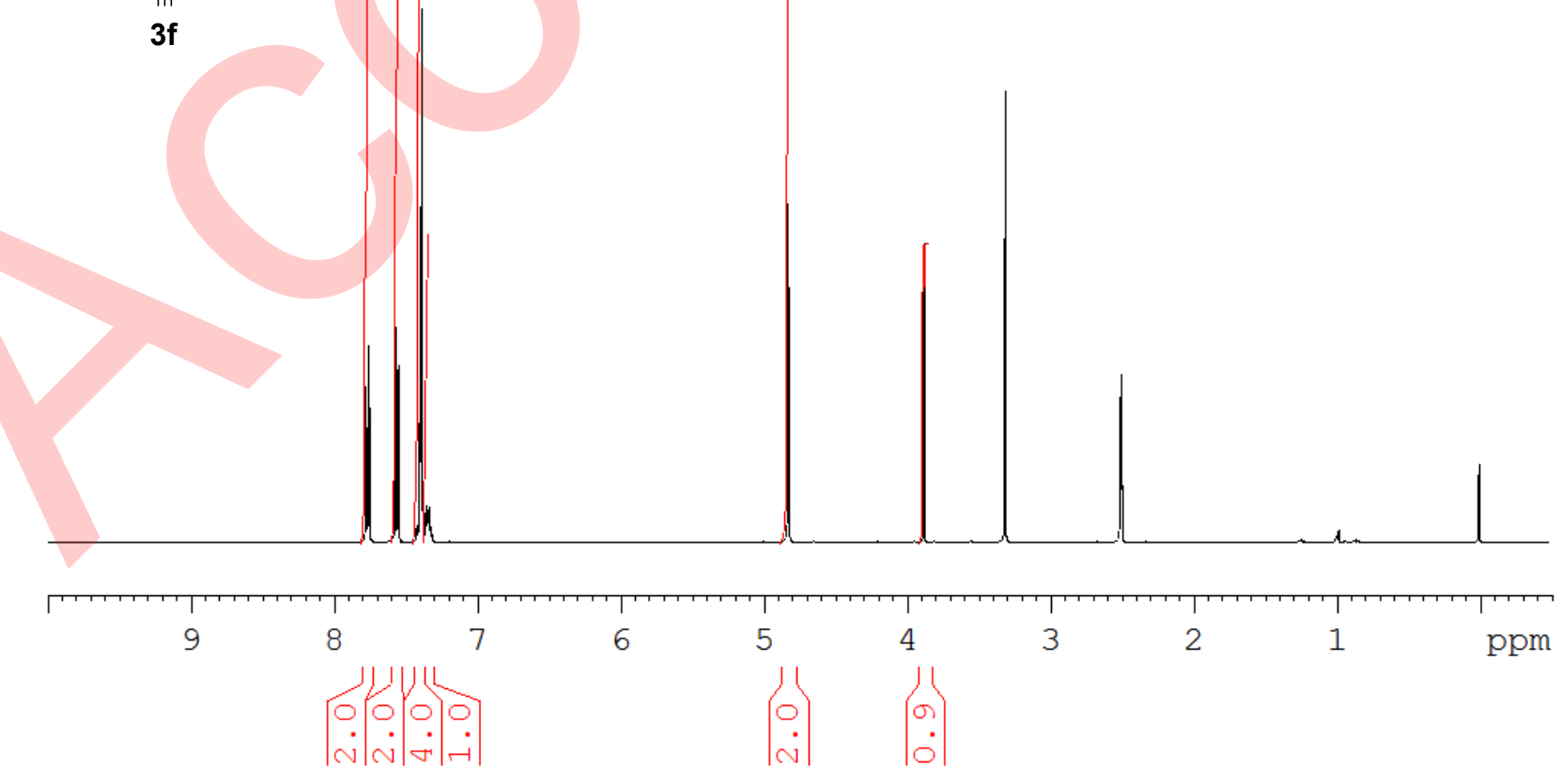



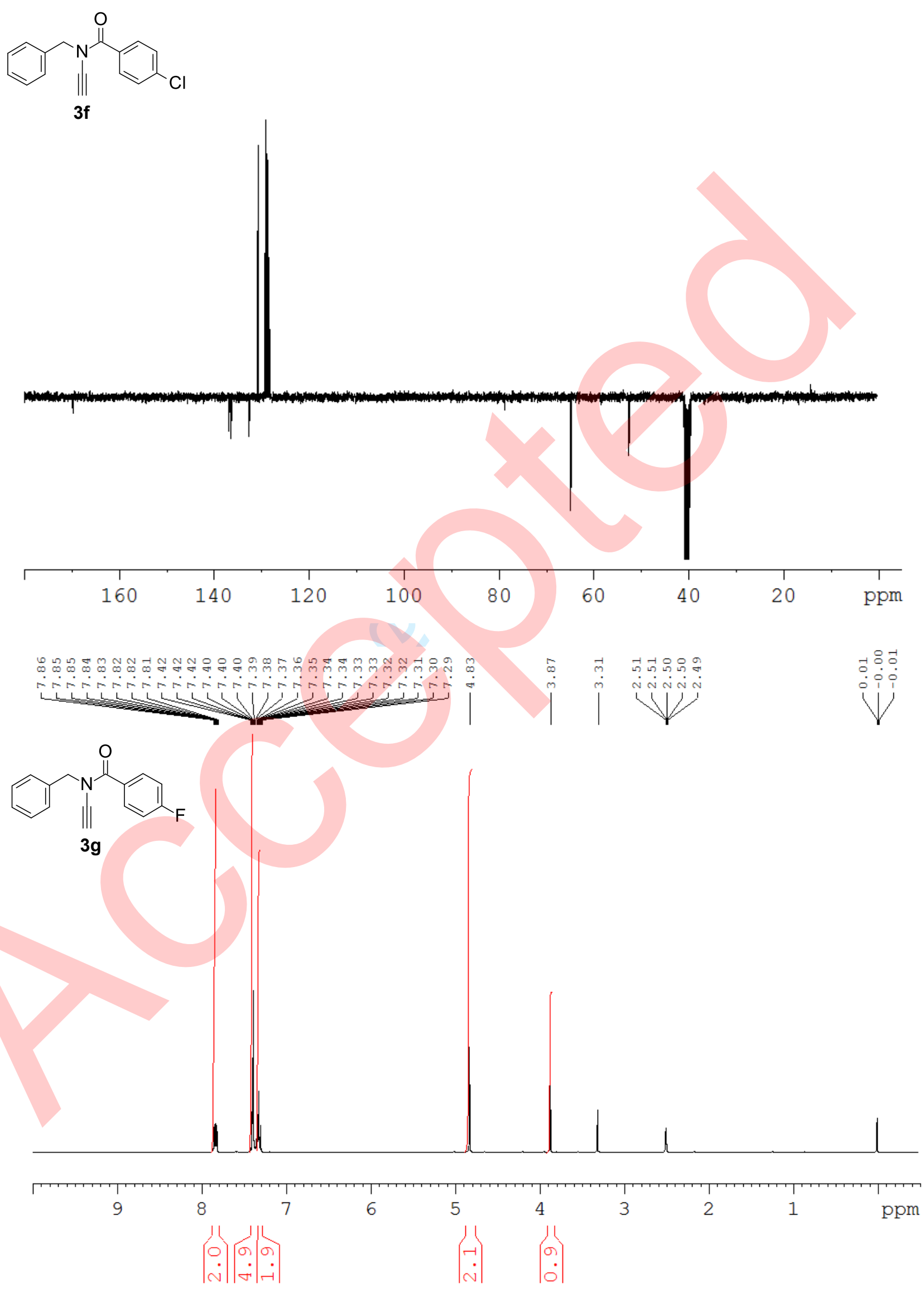

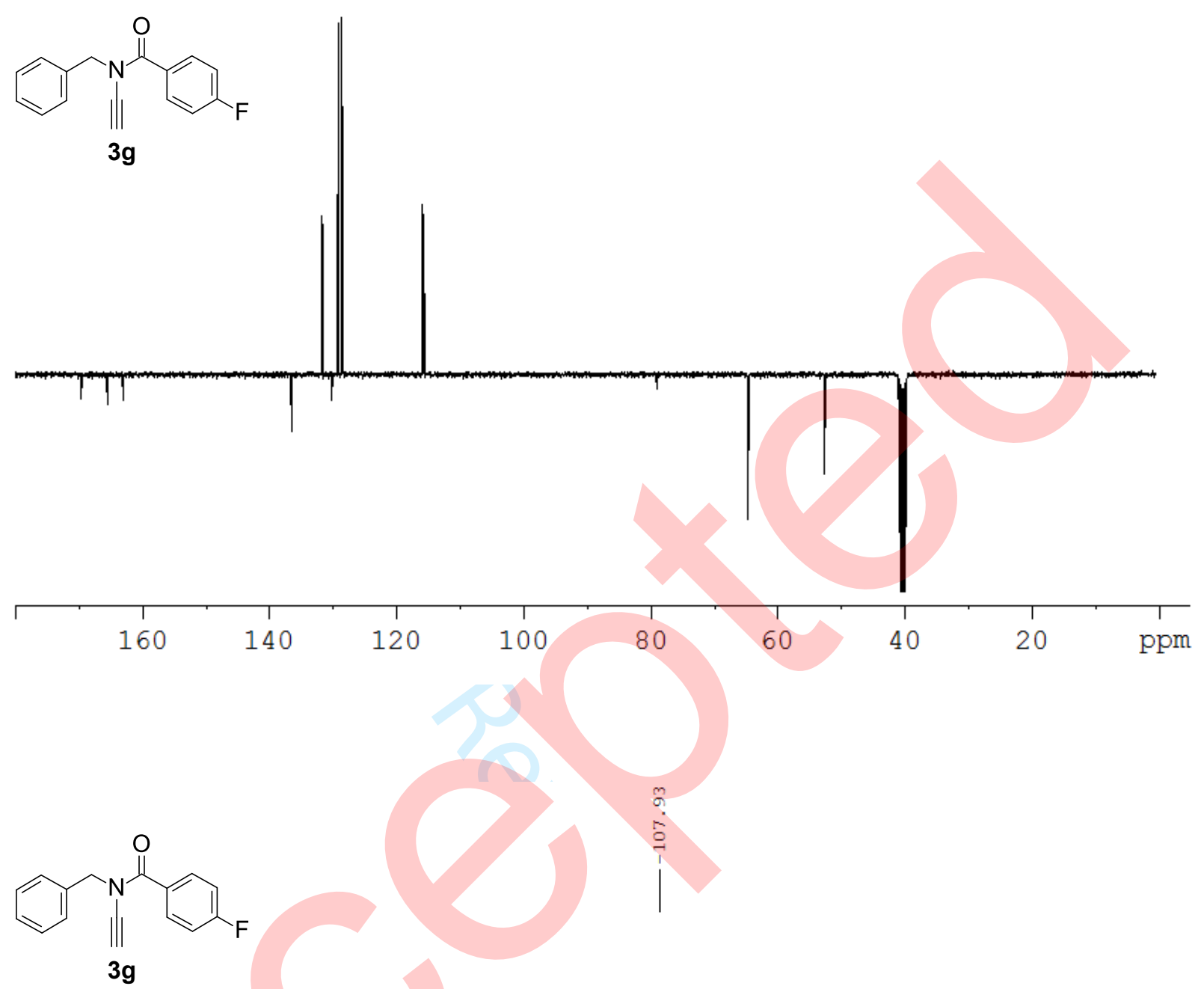

33 


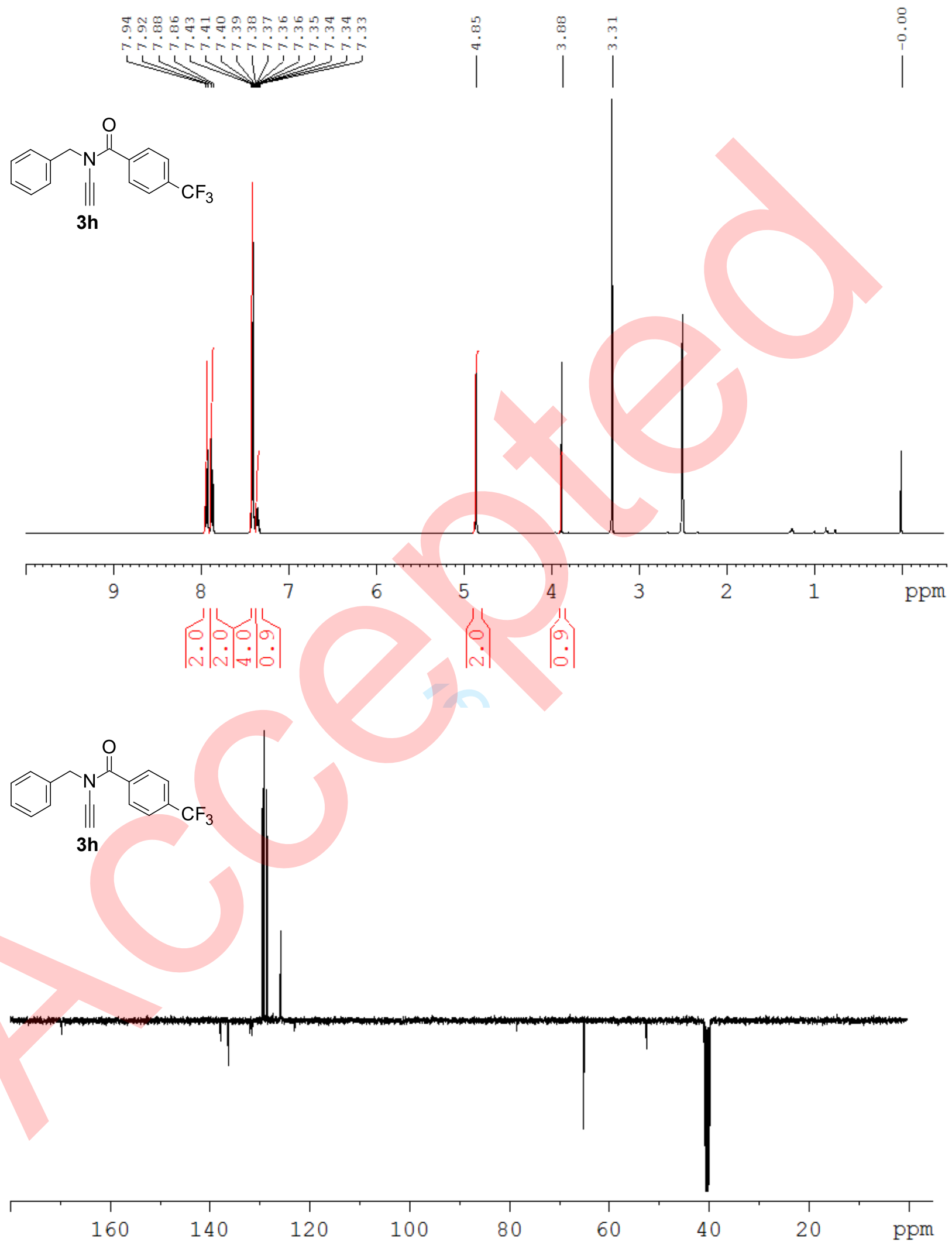



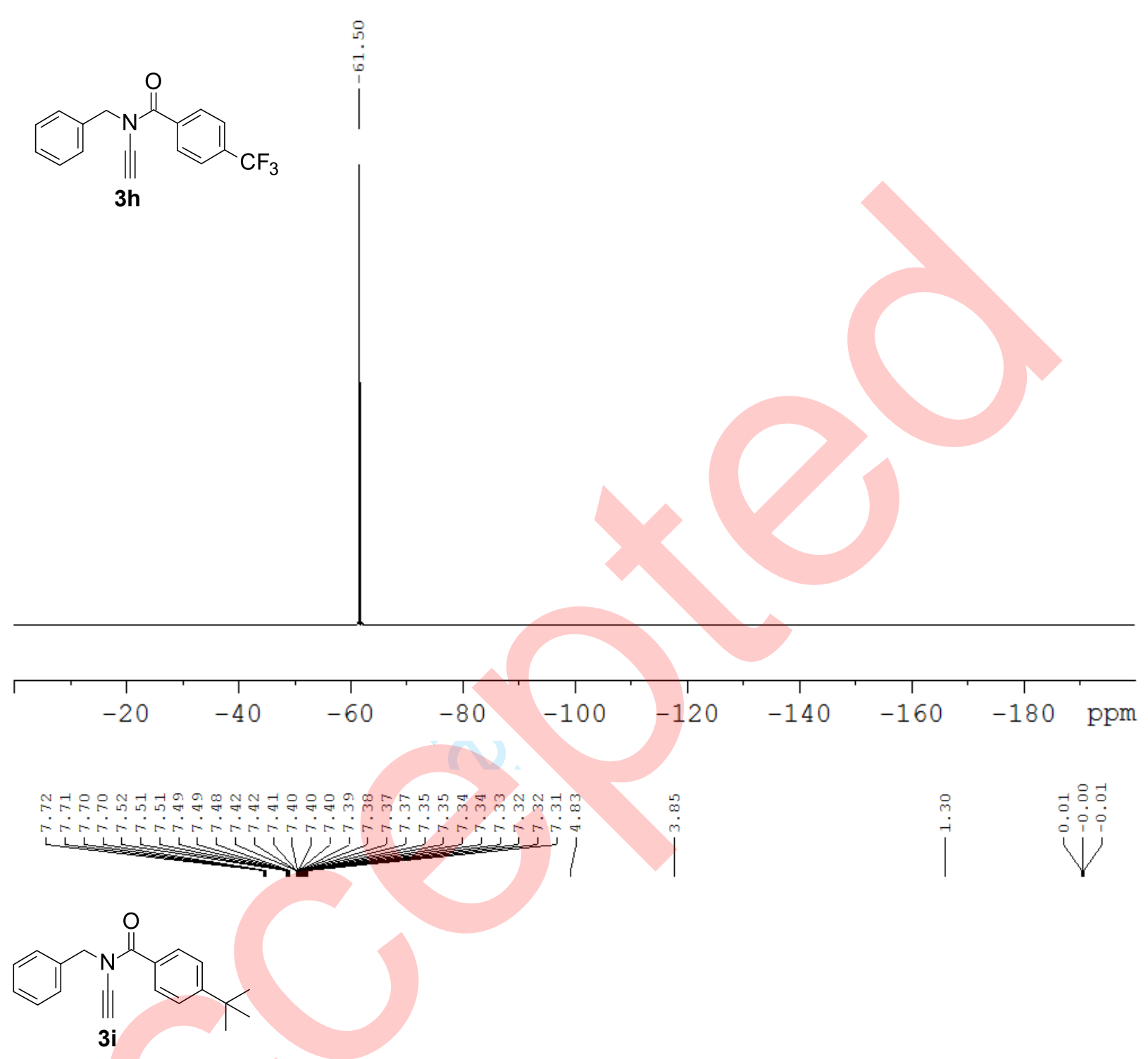

39 

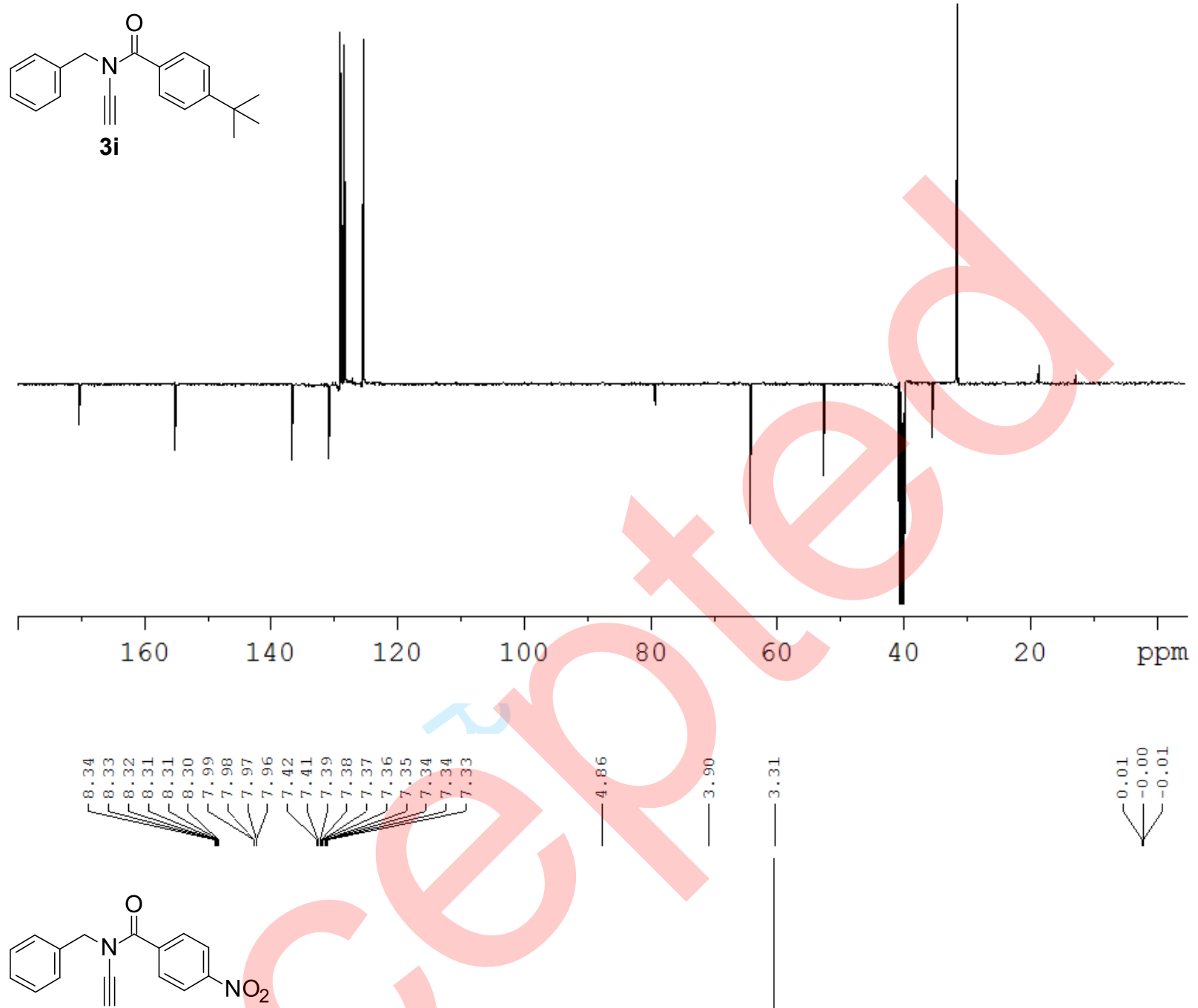

3j

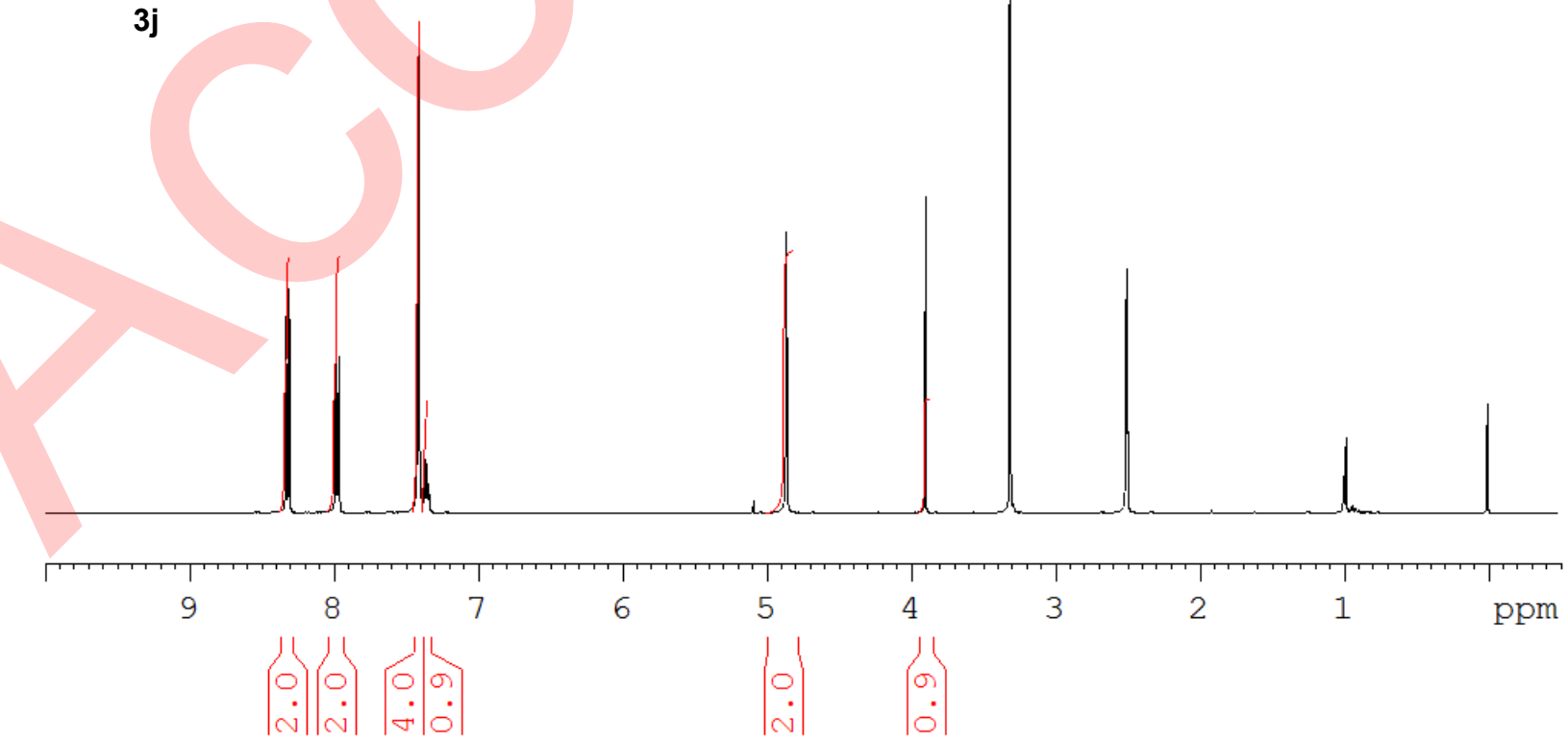



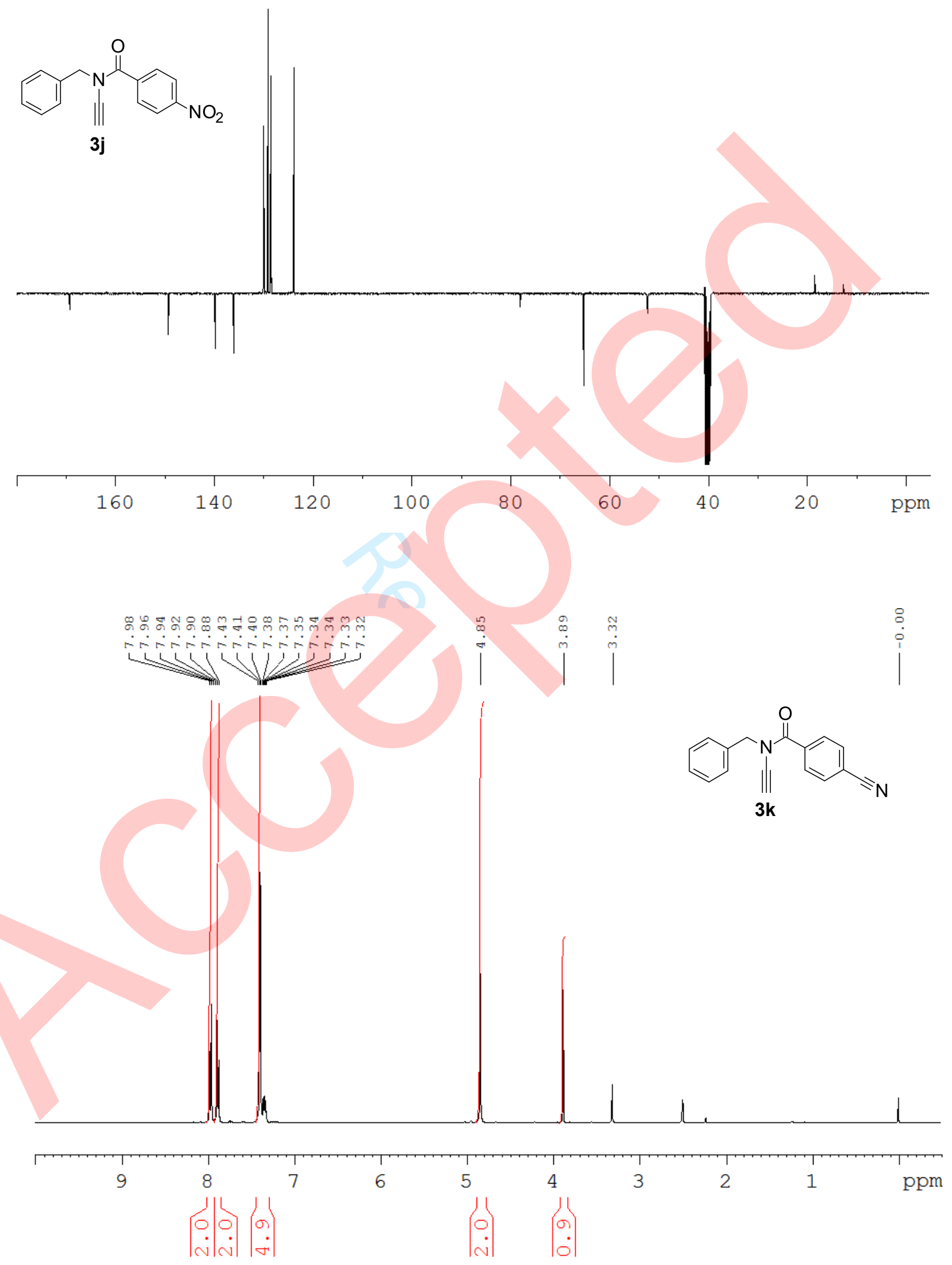

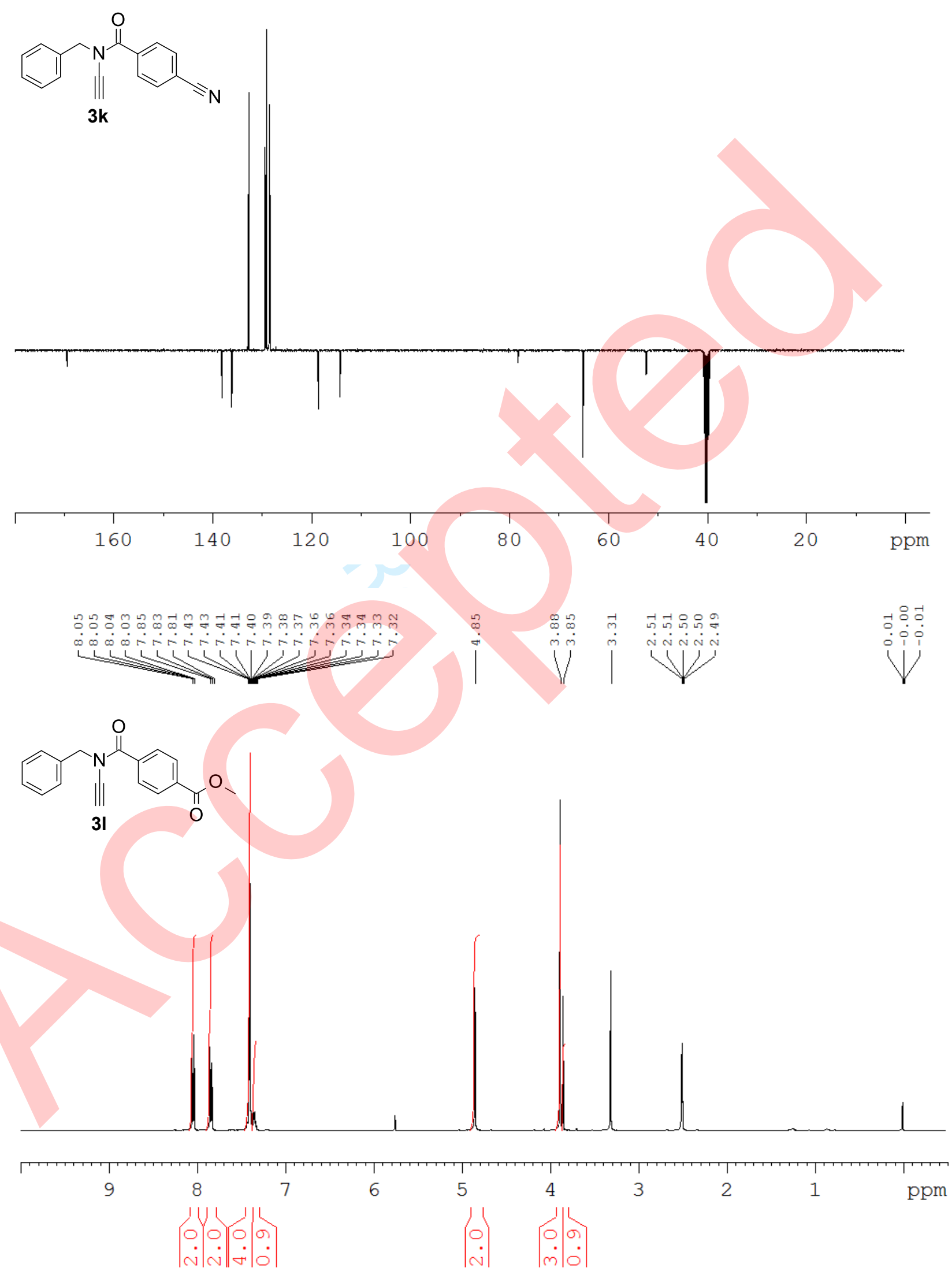

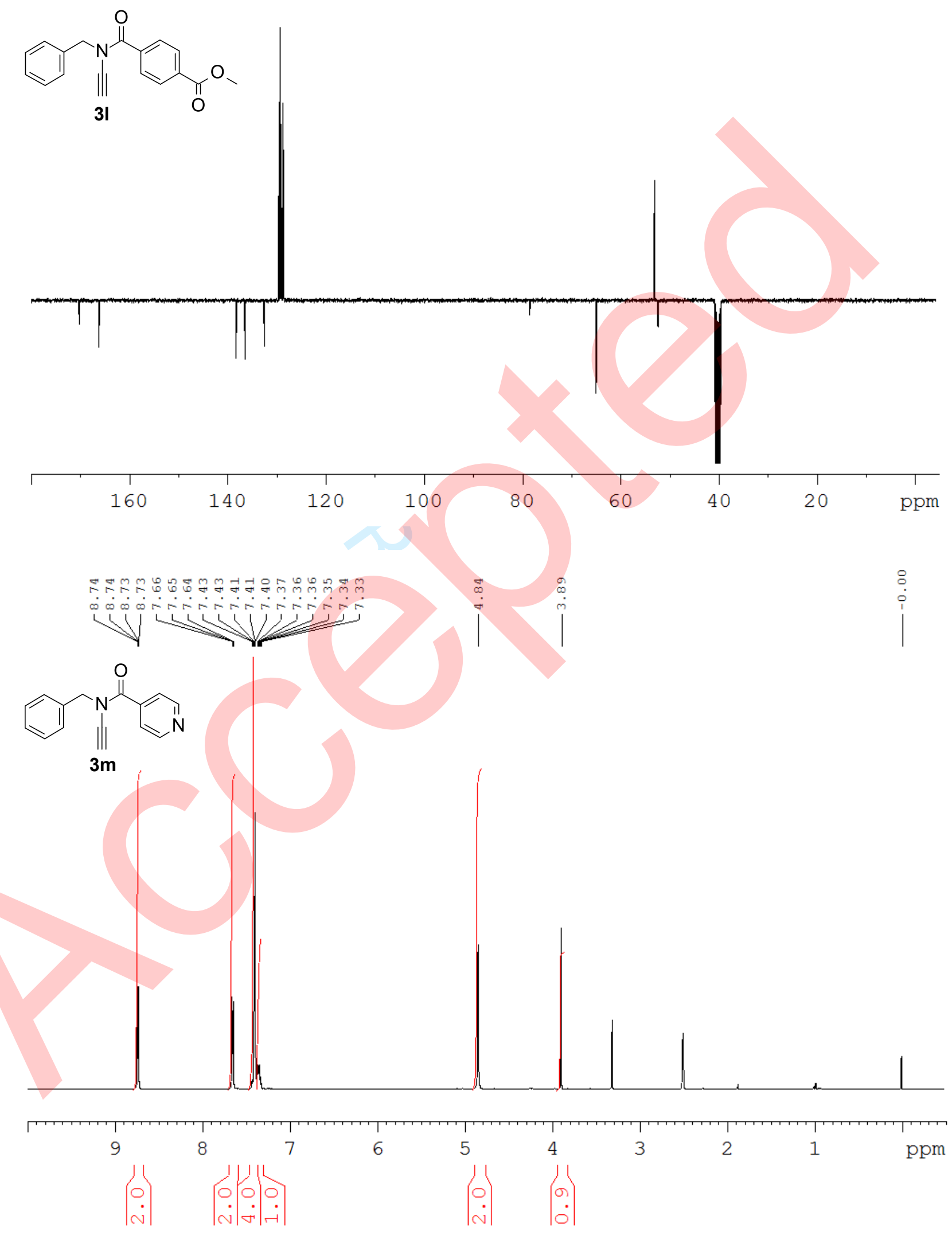

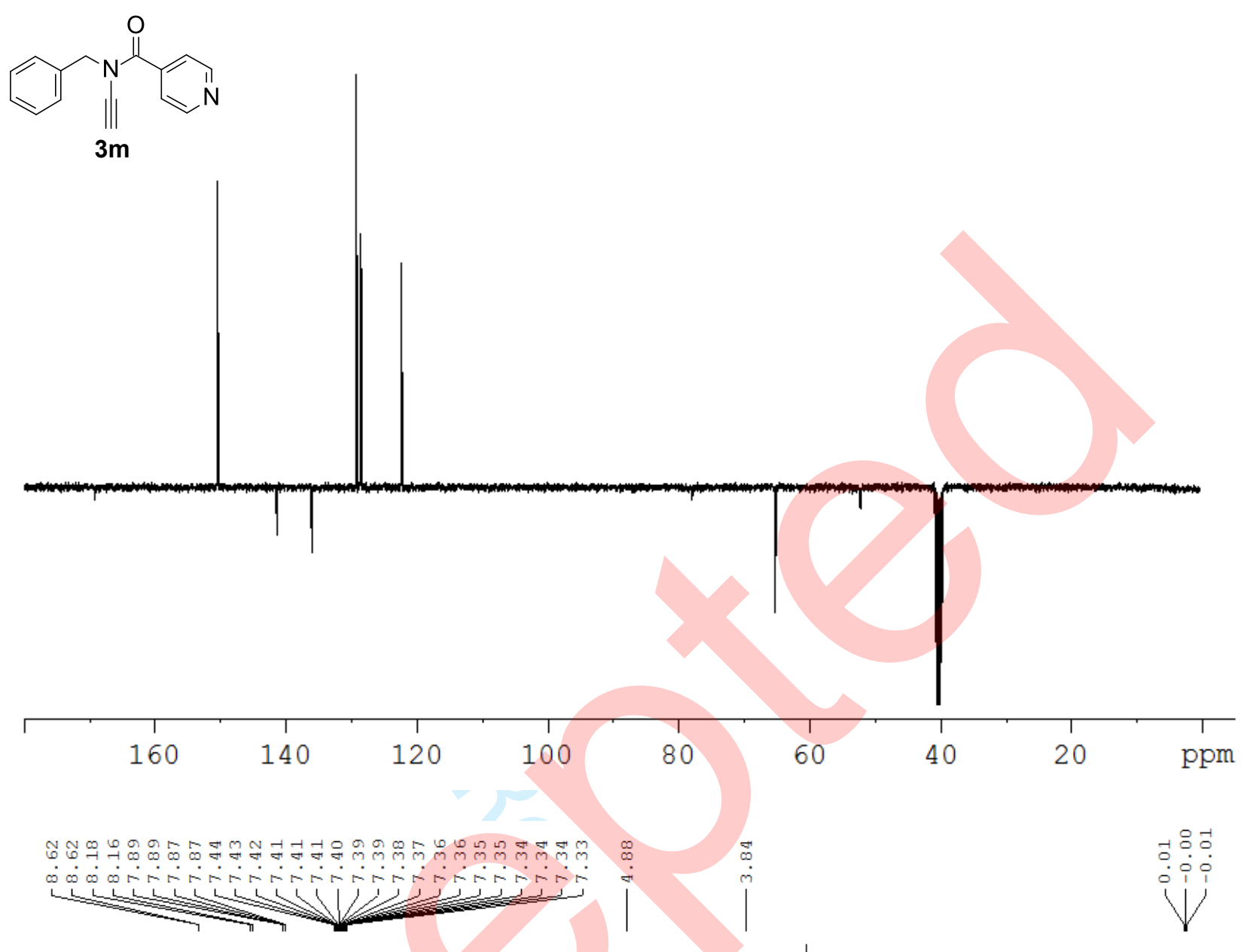<smiles>O=C(c1ccc2c(c1)SCN2)N(C#[Sn])Cc1ccccc1</smiles> 

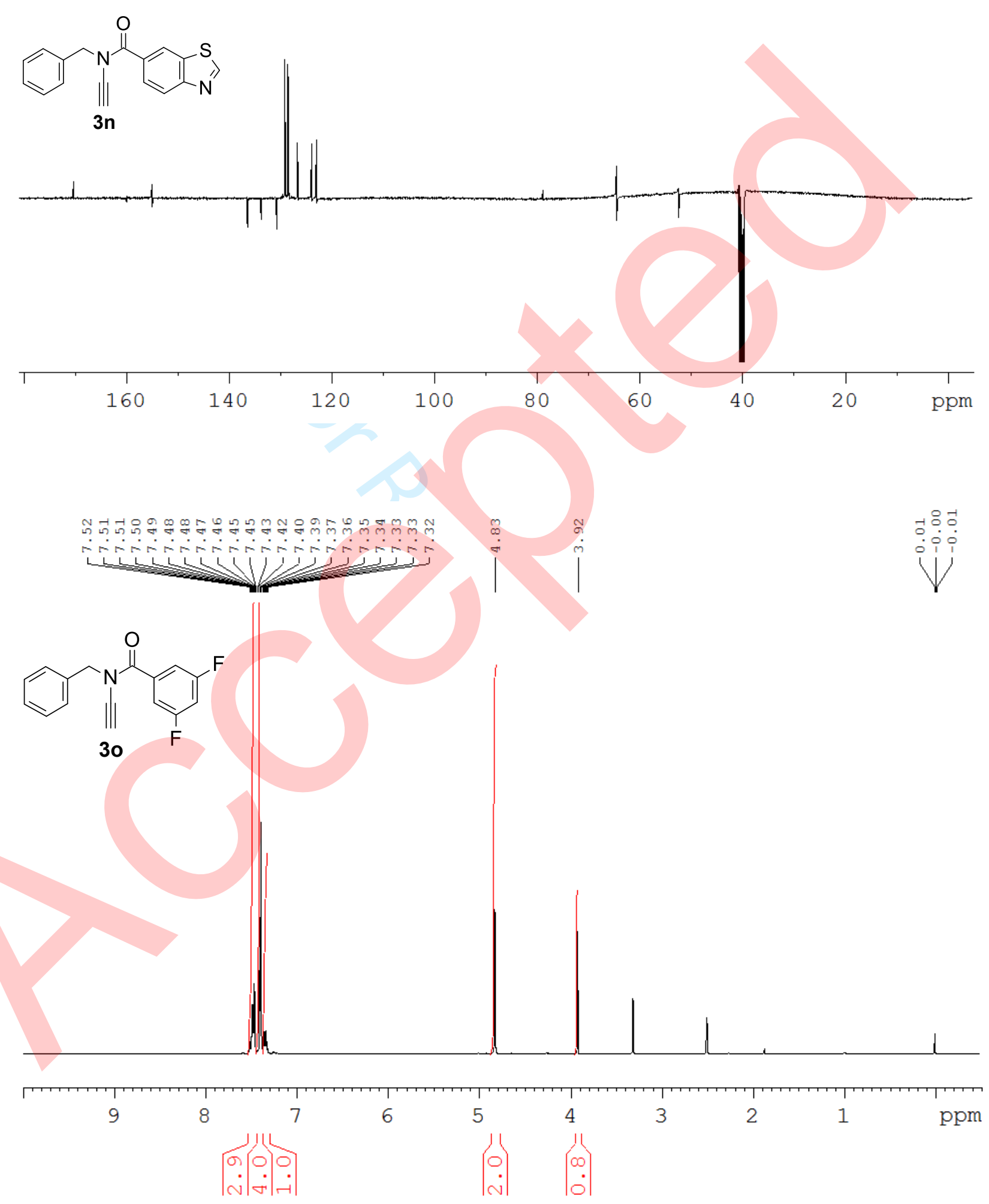

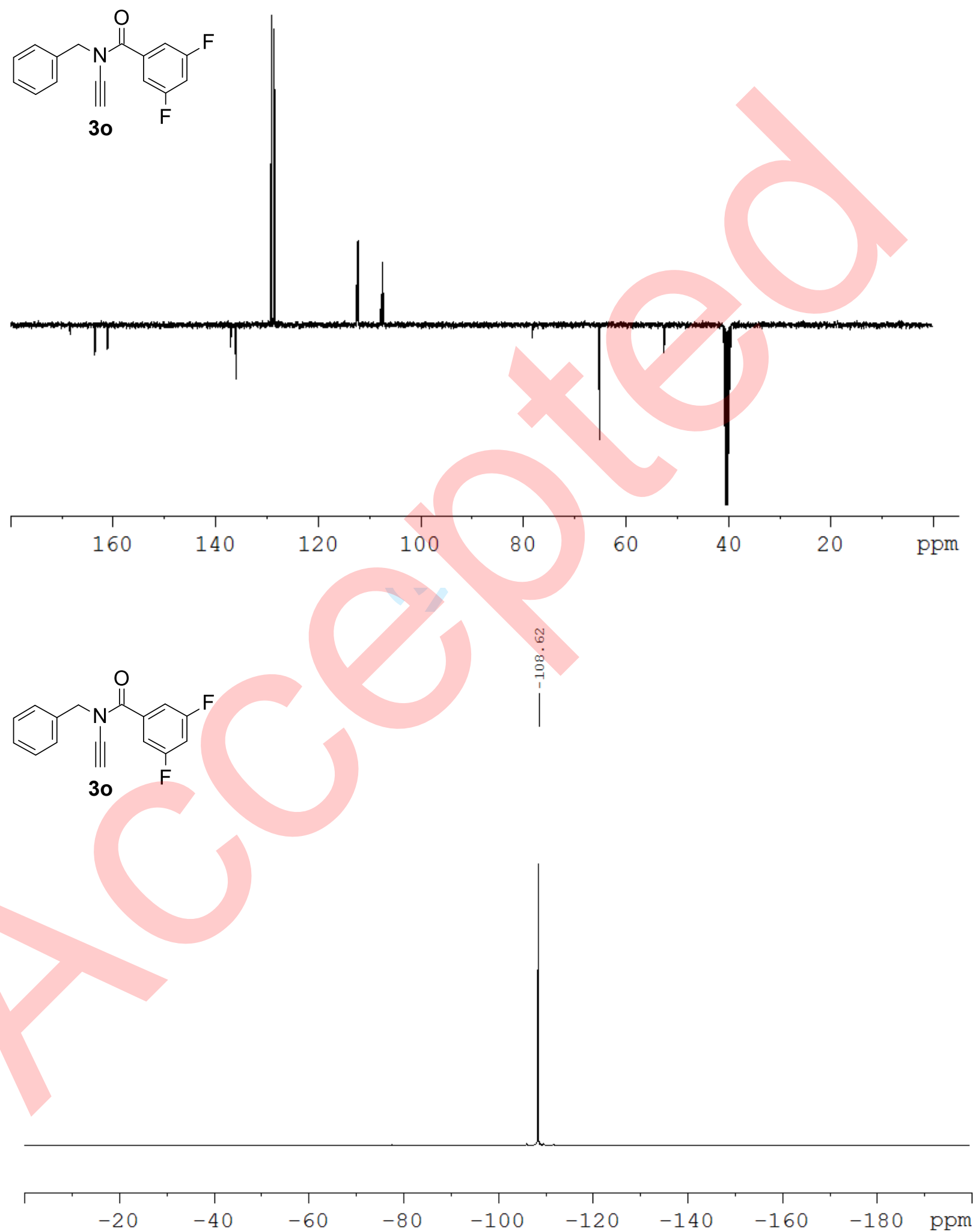

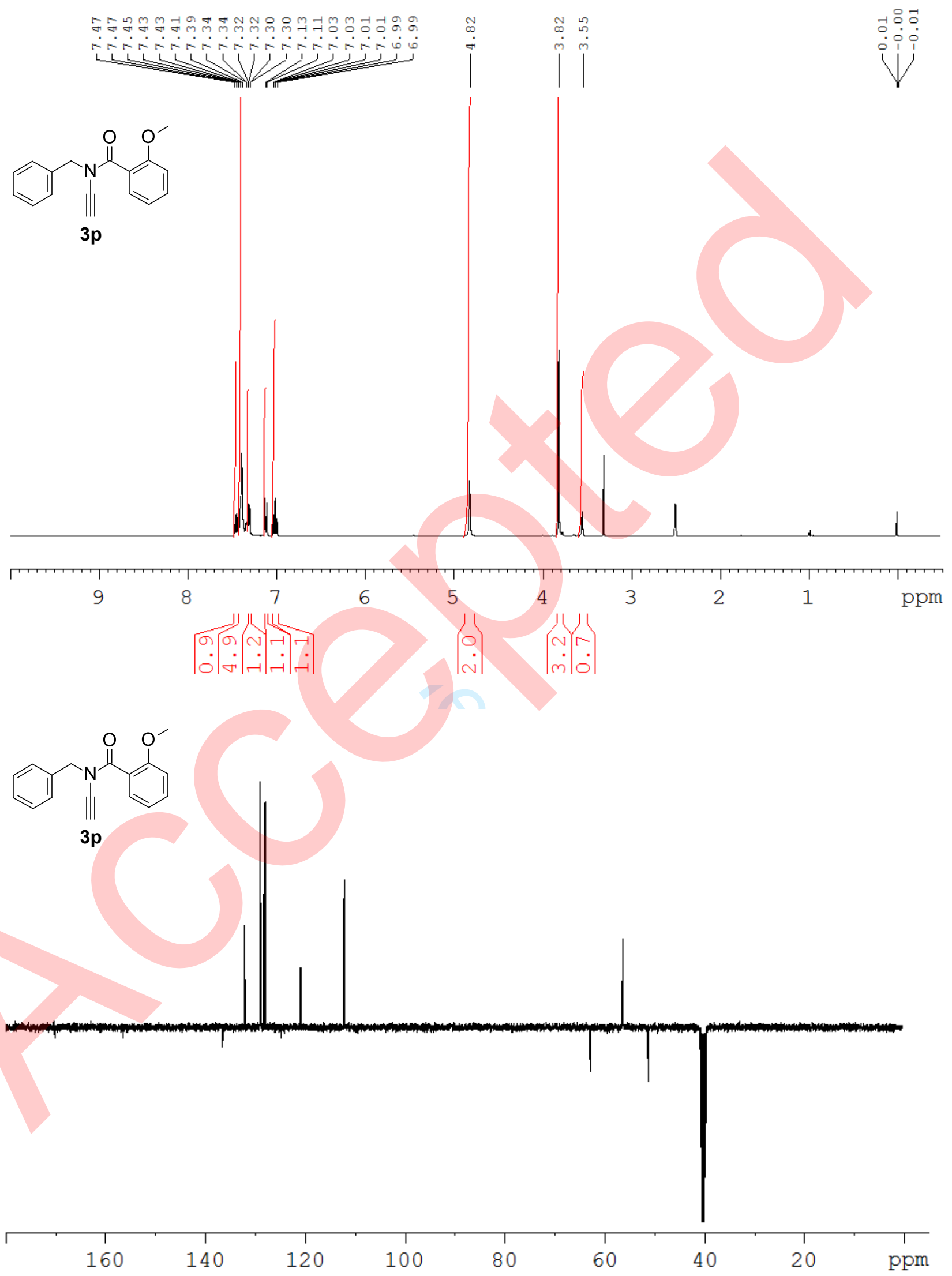


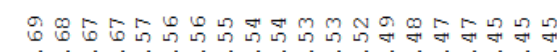

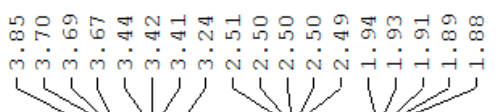

$\begin{array}{rlrl}-1 & 0 & -1 \\ 0 & 0 \\ 0 & 0 & 0 \\ 0 & 0 & 0\end{array}$

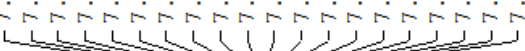

4<smiles>COCCCN(C#C[13C](=O)c1ccccc1)C(=O)c1ccccc1</smiles>
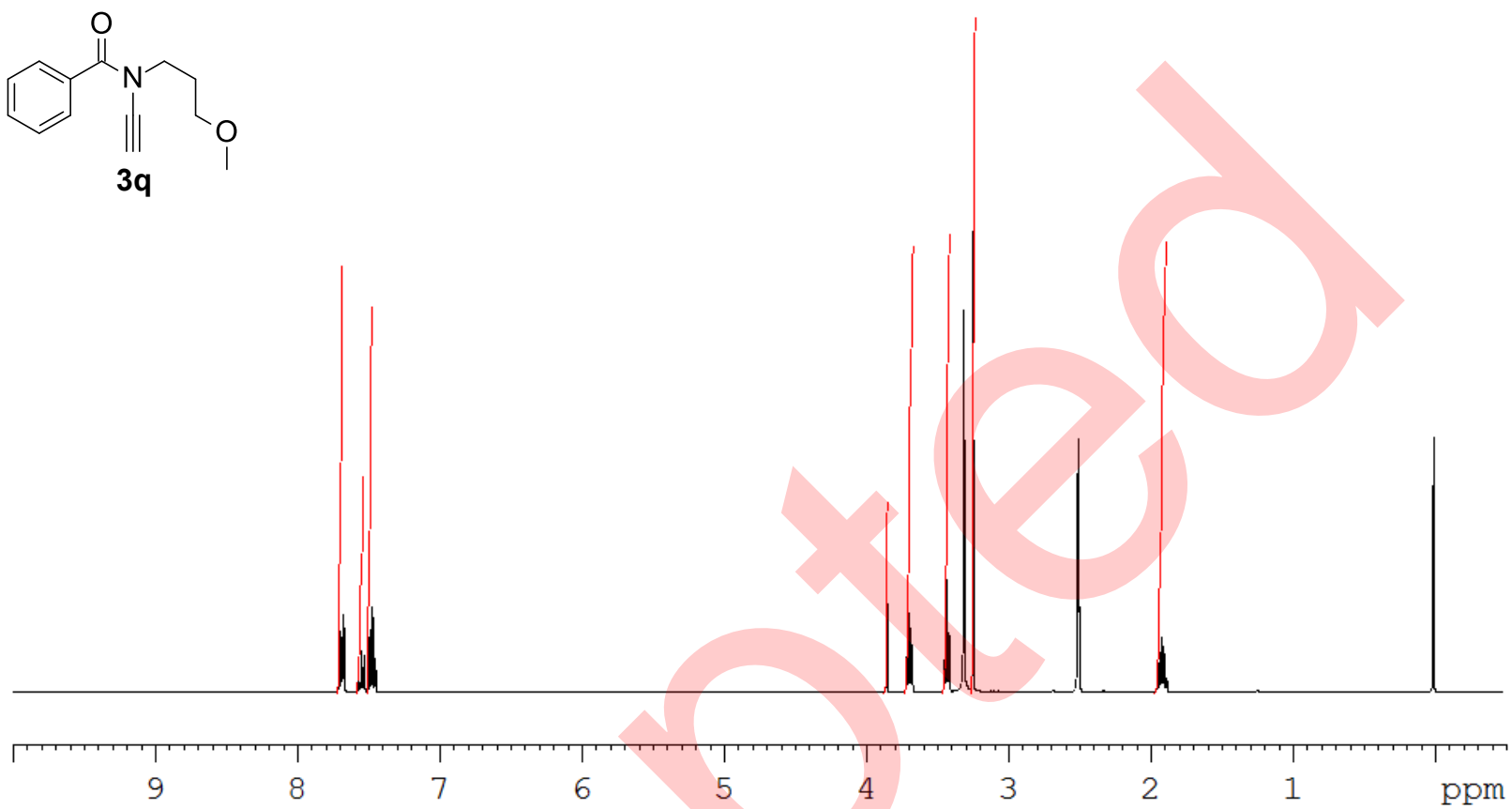

54

43

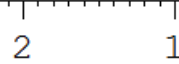

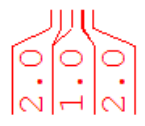

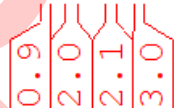

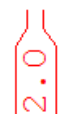

ppm
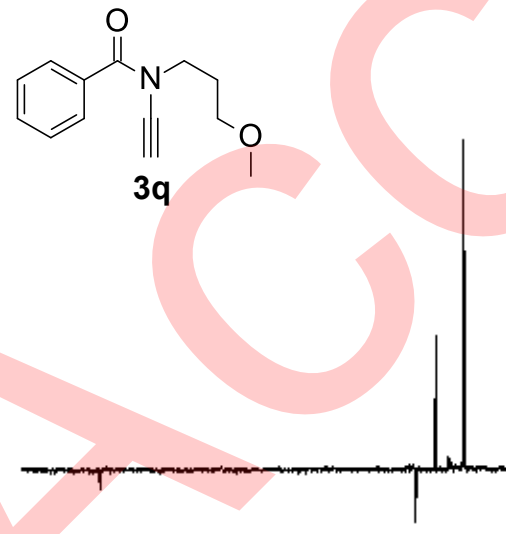

53
4
5
6
7
8
9

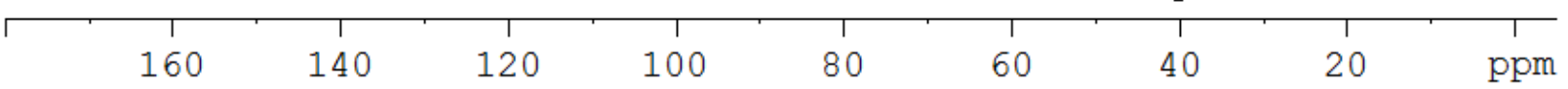




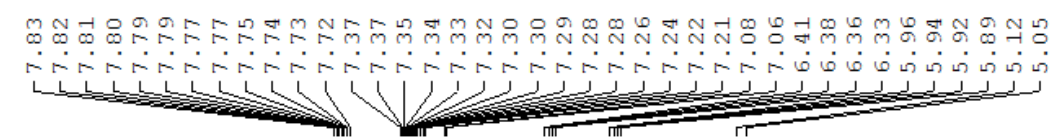<smiles>O=C1c2ccccc2/C(=C\C(F)(F)F)N1Cc1ccccc1</smiles>

$4 a$
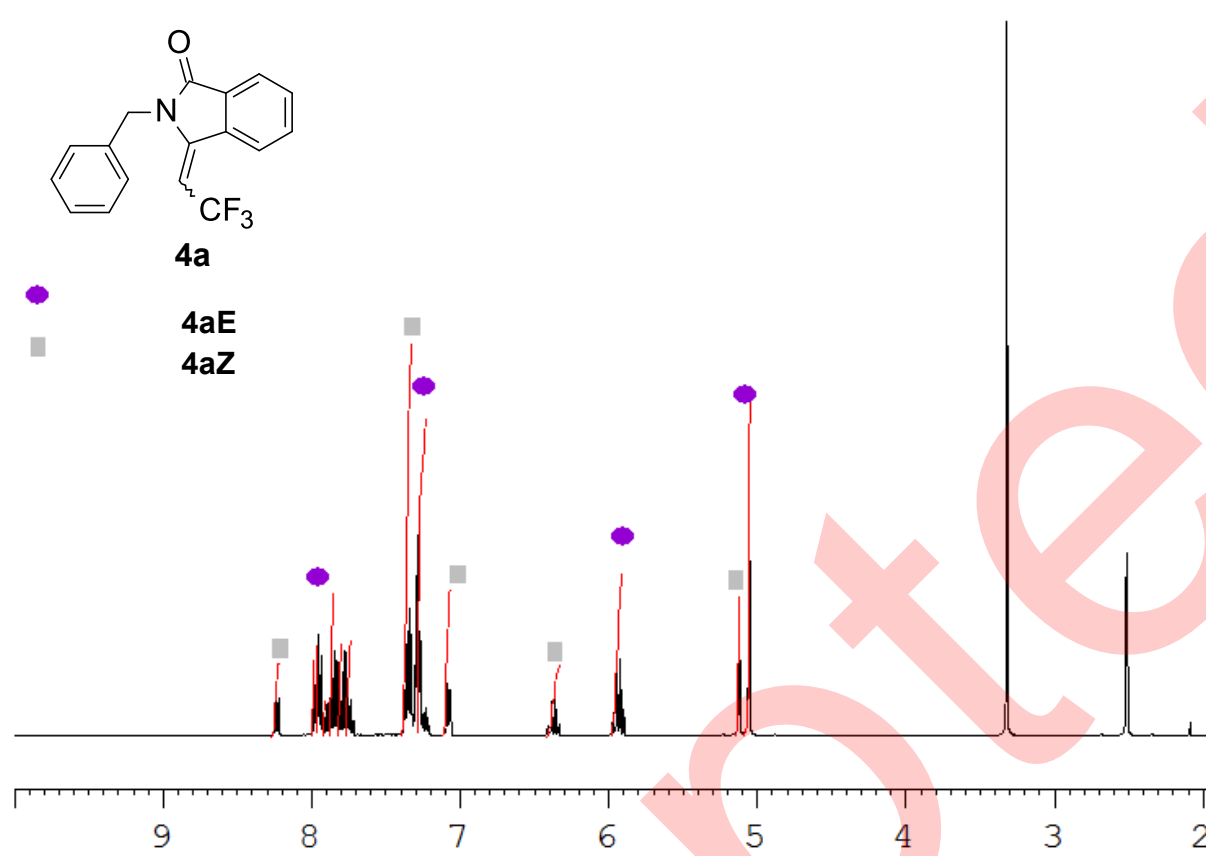

21

ppm

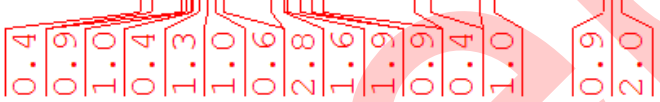<smiles>O=C1c2ccccc2/C(=C\C(F)(F)F)N1Cc1ccccc1</smiles>

$4 a$

- 4aE

$4 \mathrm{aZ}$

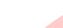

$4 a z$
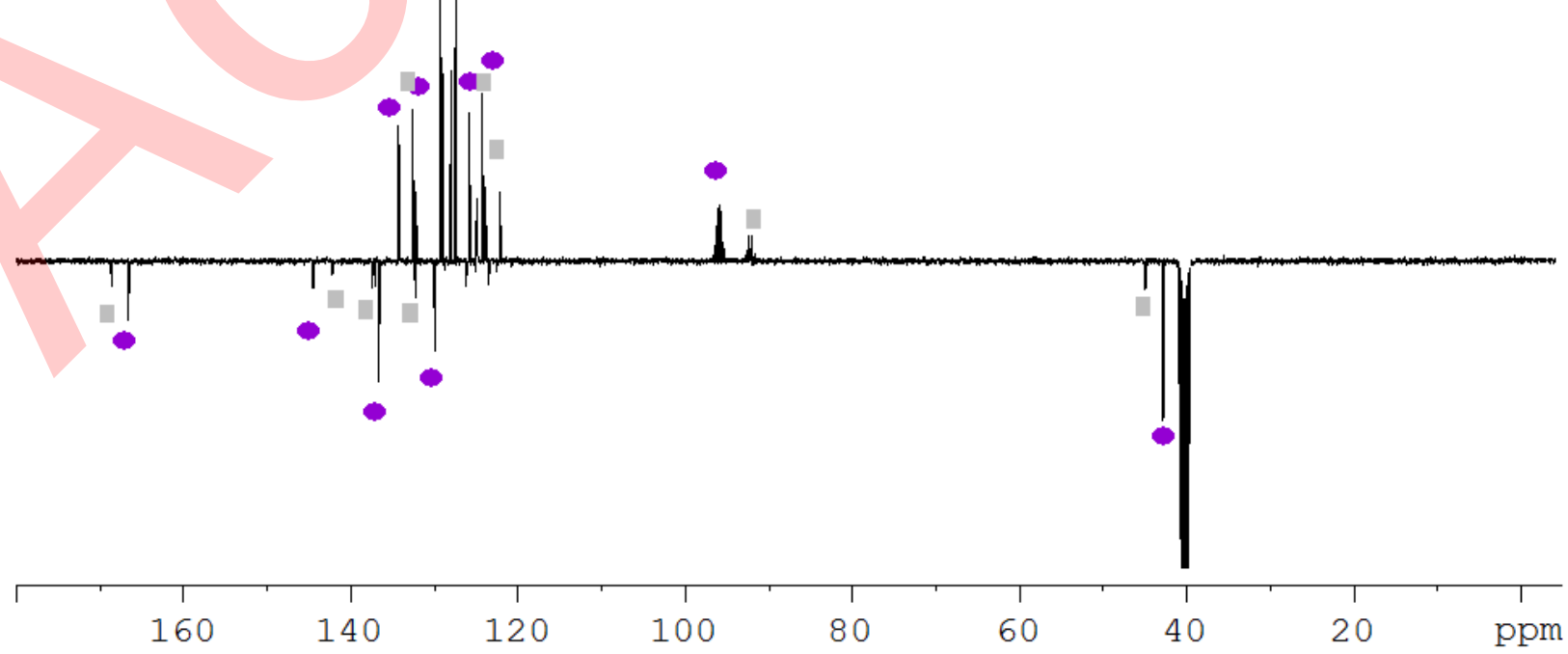


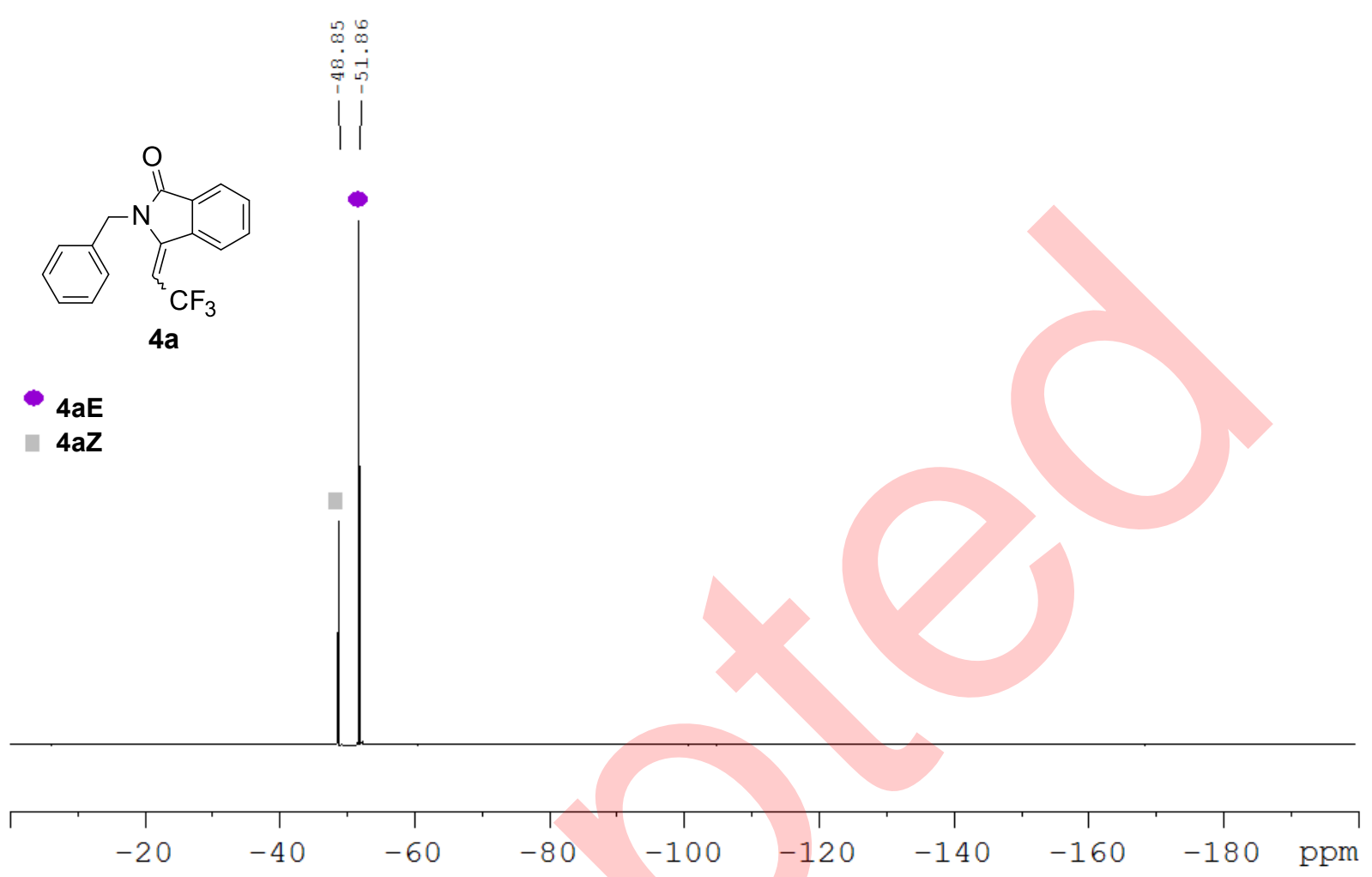

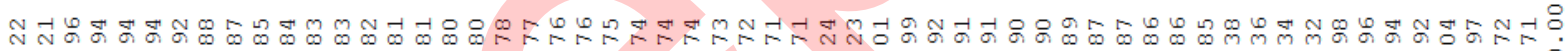
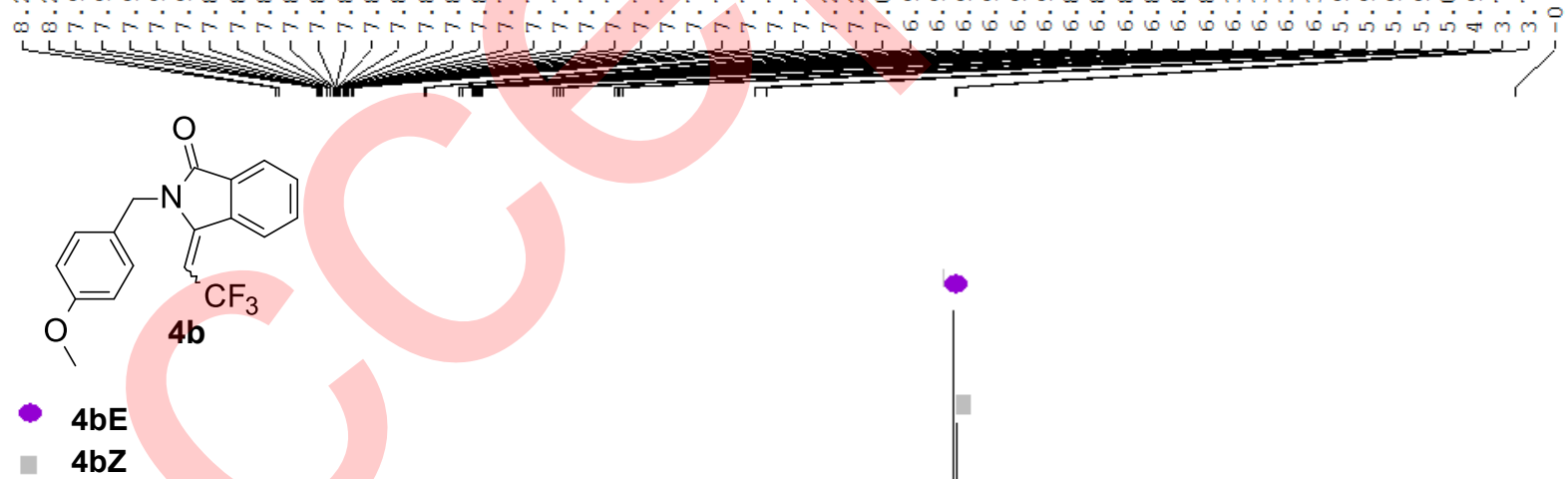

$4 b Z$

42
44
45
46
47
48
49
50
51
52
53
54
55
56
57
58
59

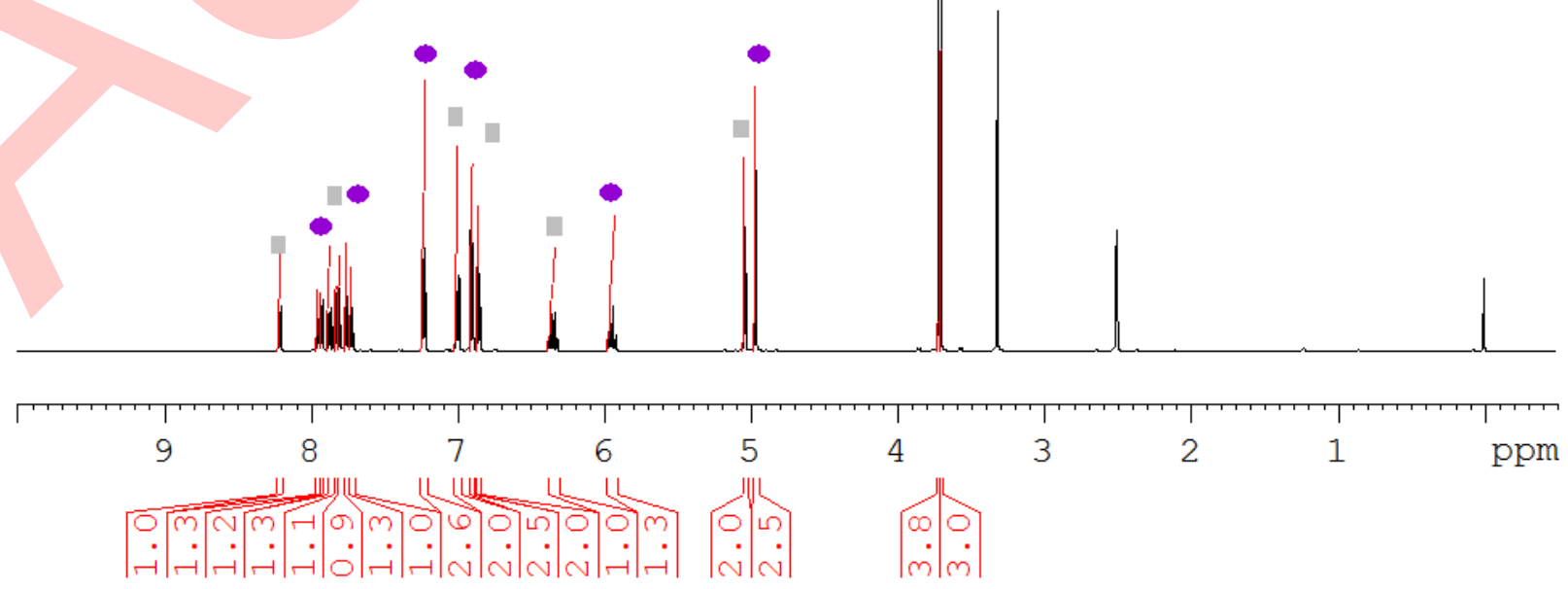



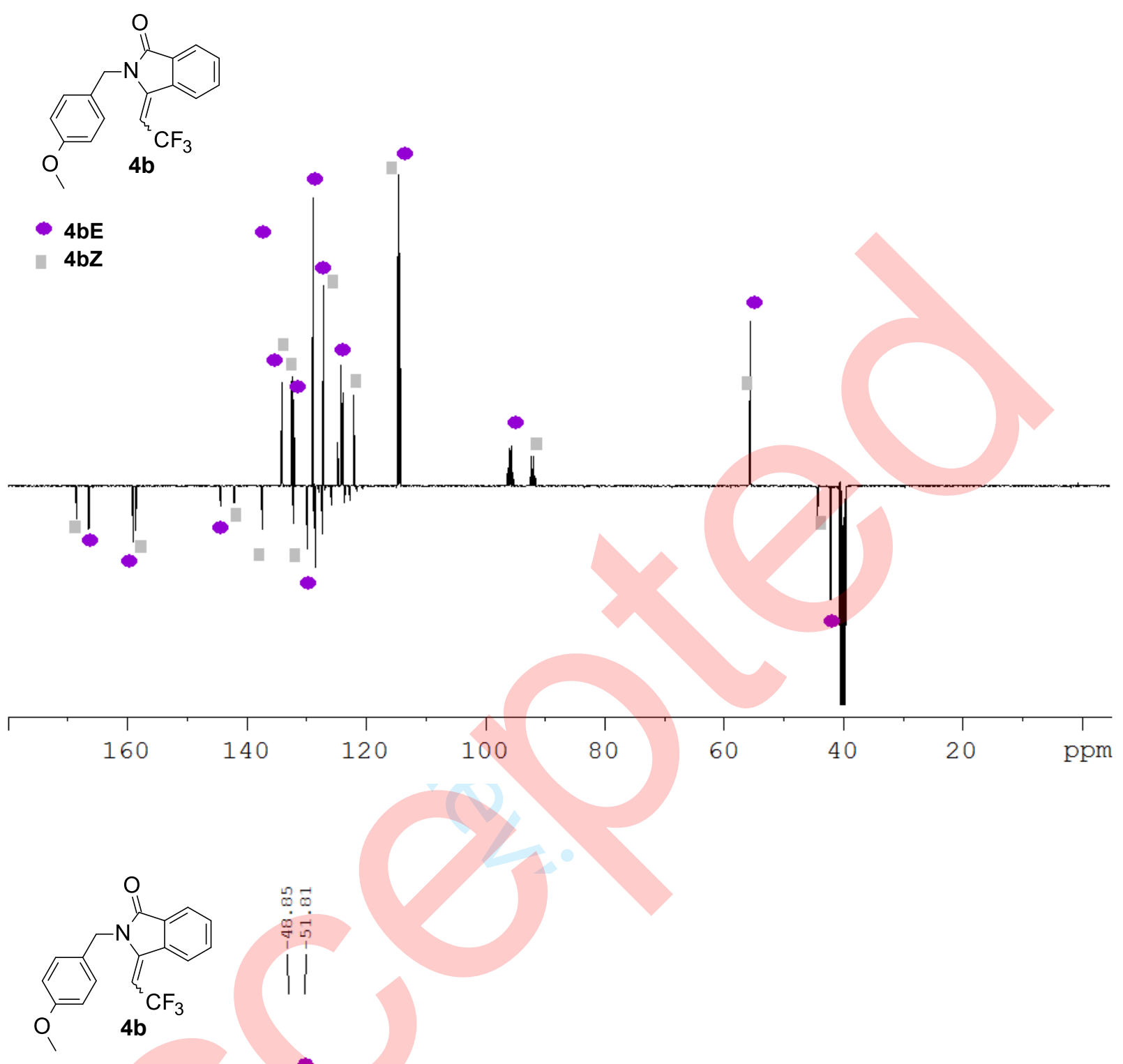

- 4bE

다 $4 \mathrm{bZ}$
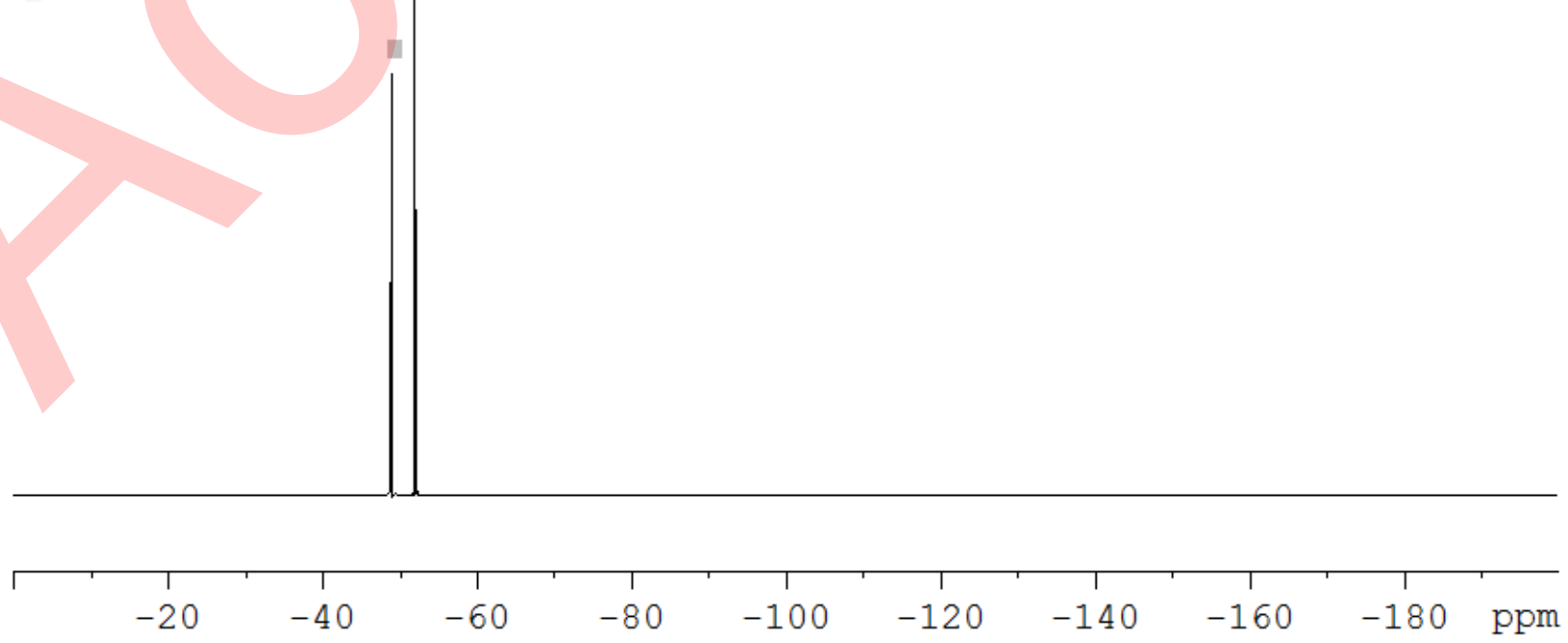


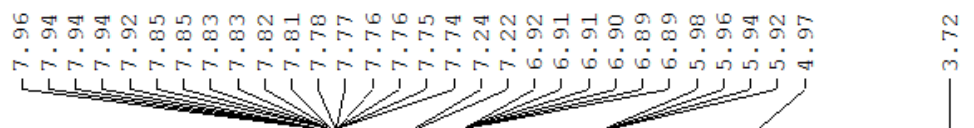<smiles>COc1ccc(CN2C(=O)c3ccccc3/C2=C\C=C\C(=O)[O-])cc1</smiles>
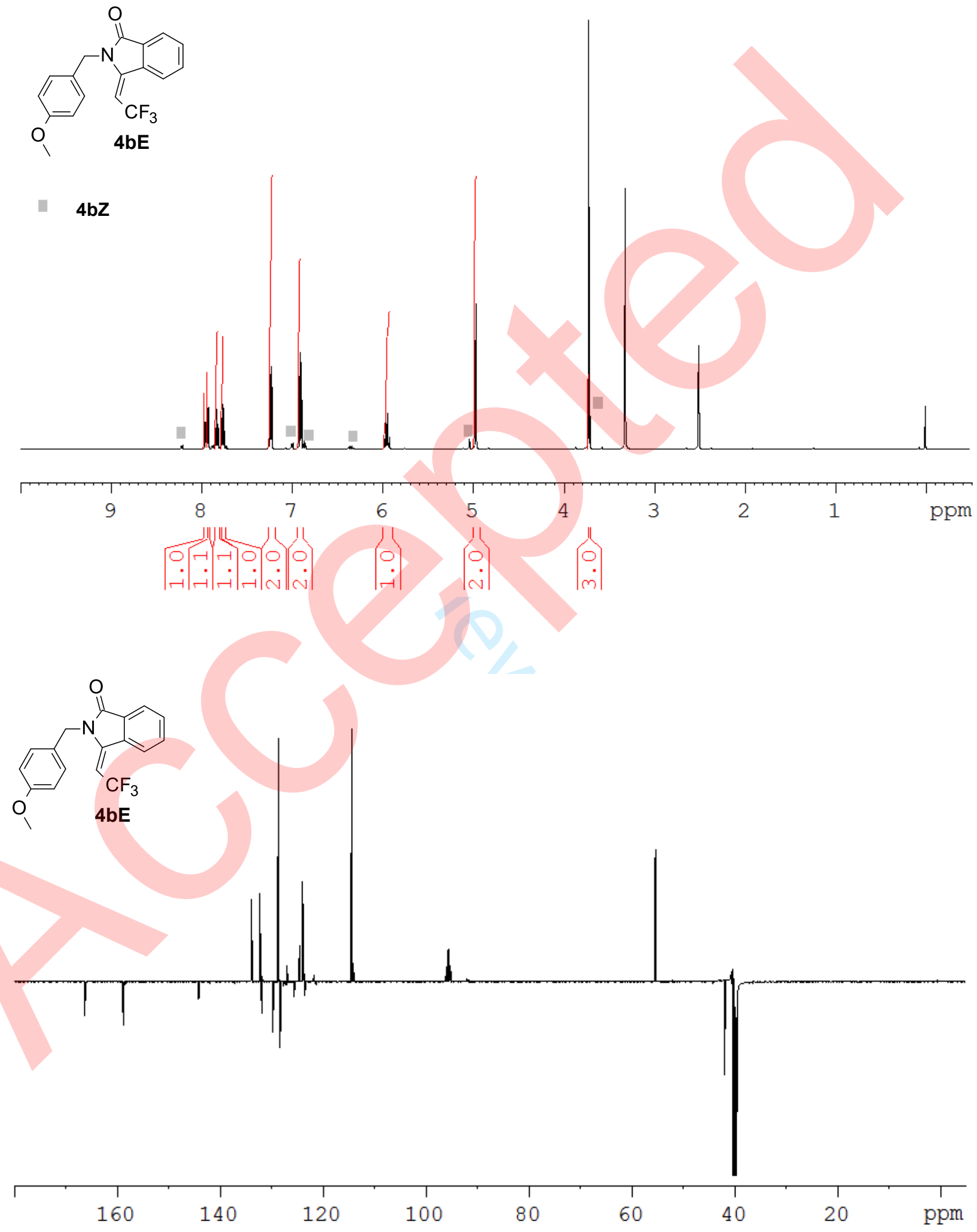


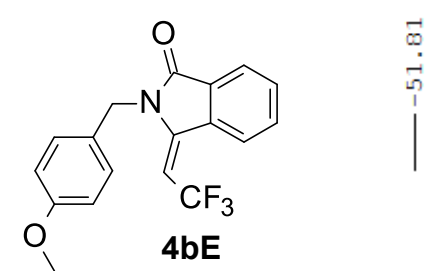
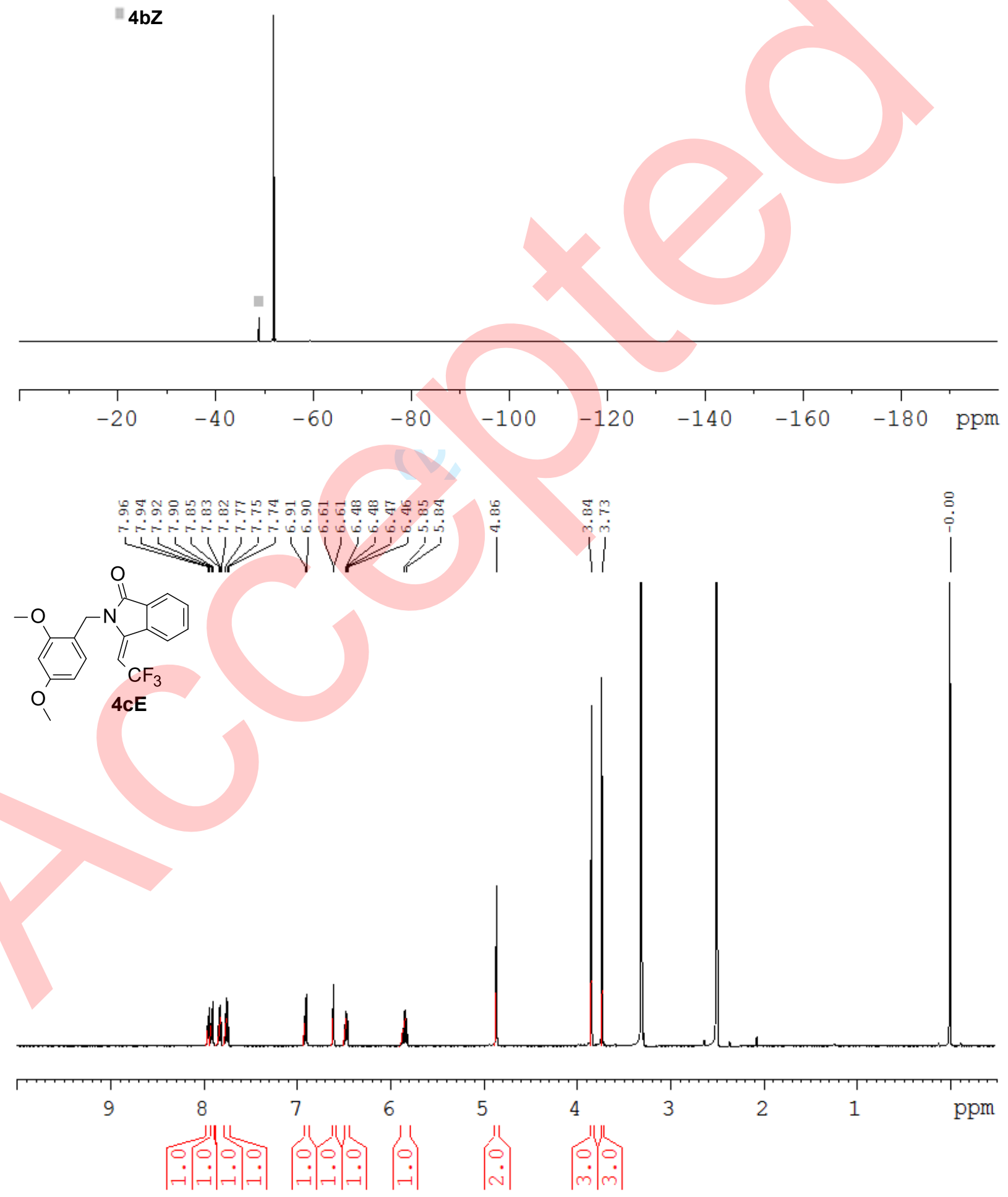

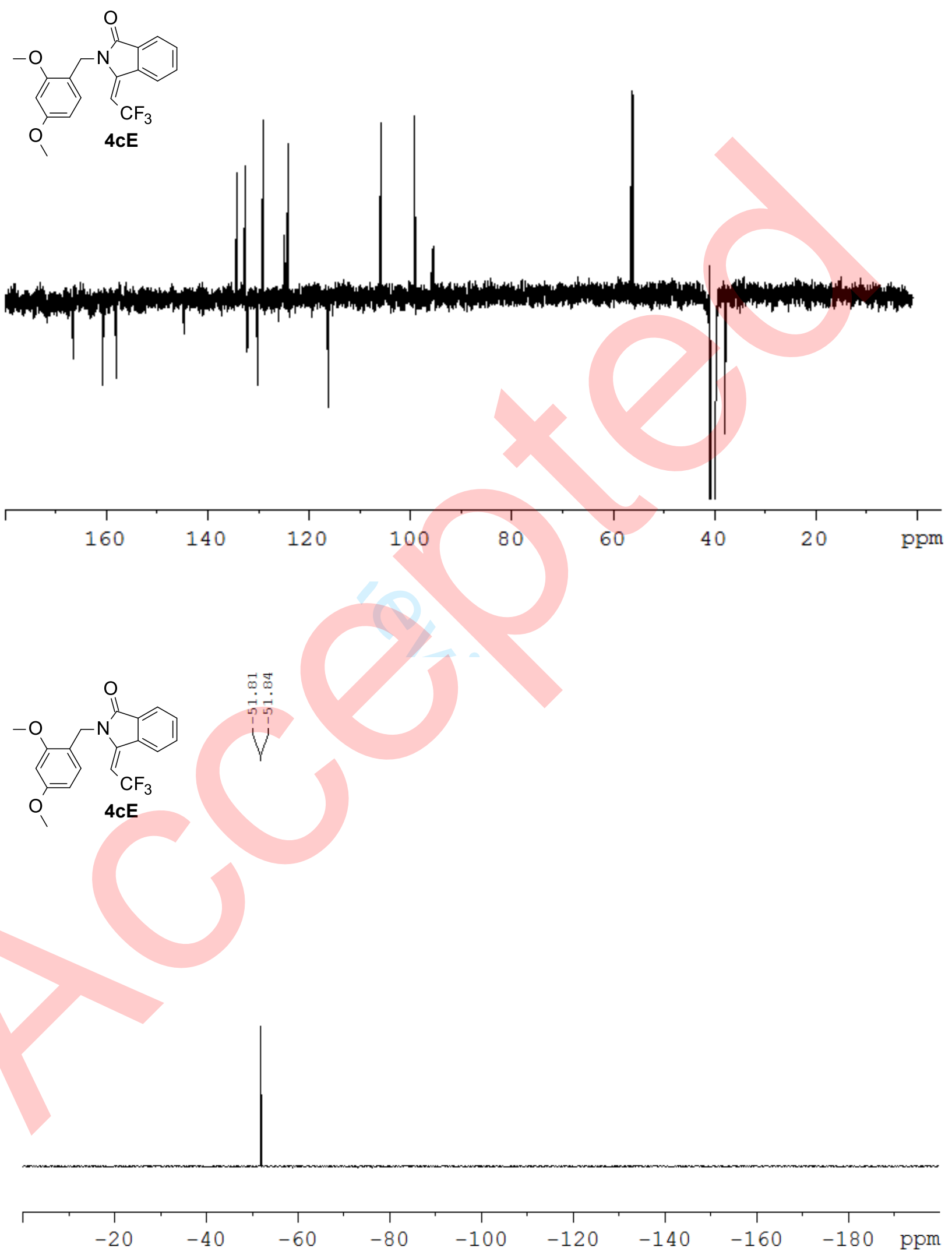


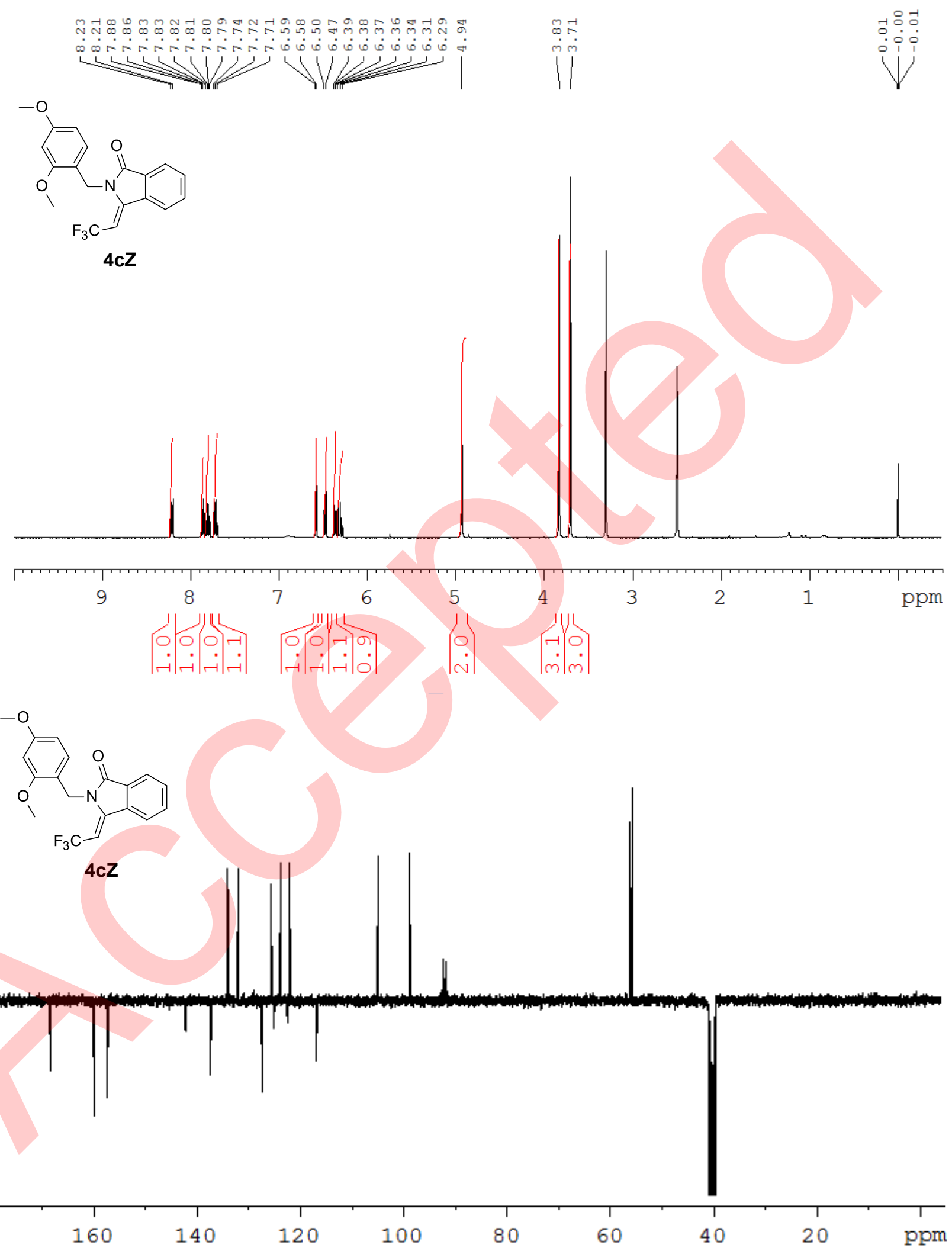



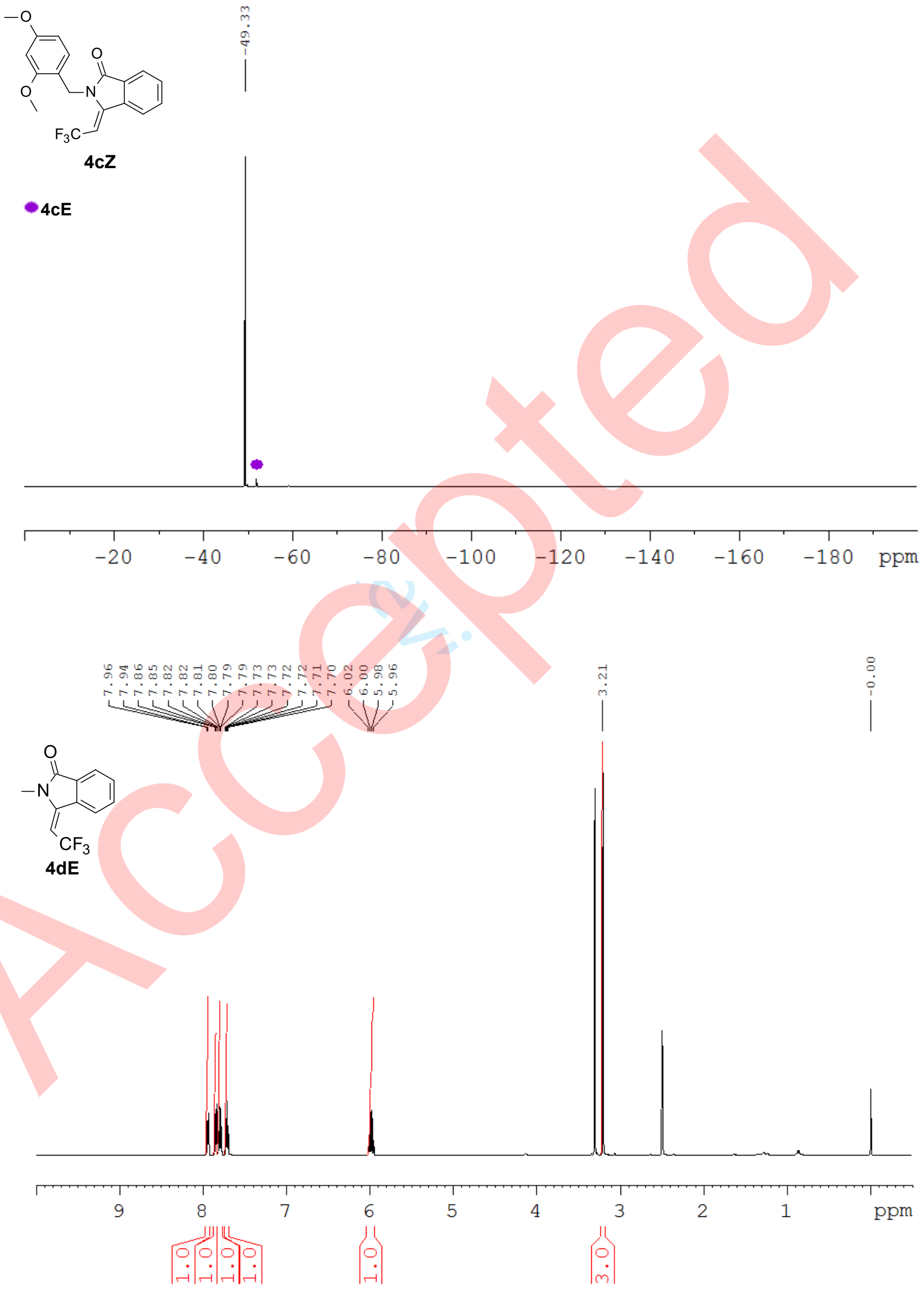

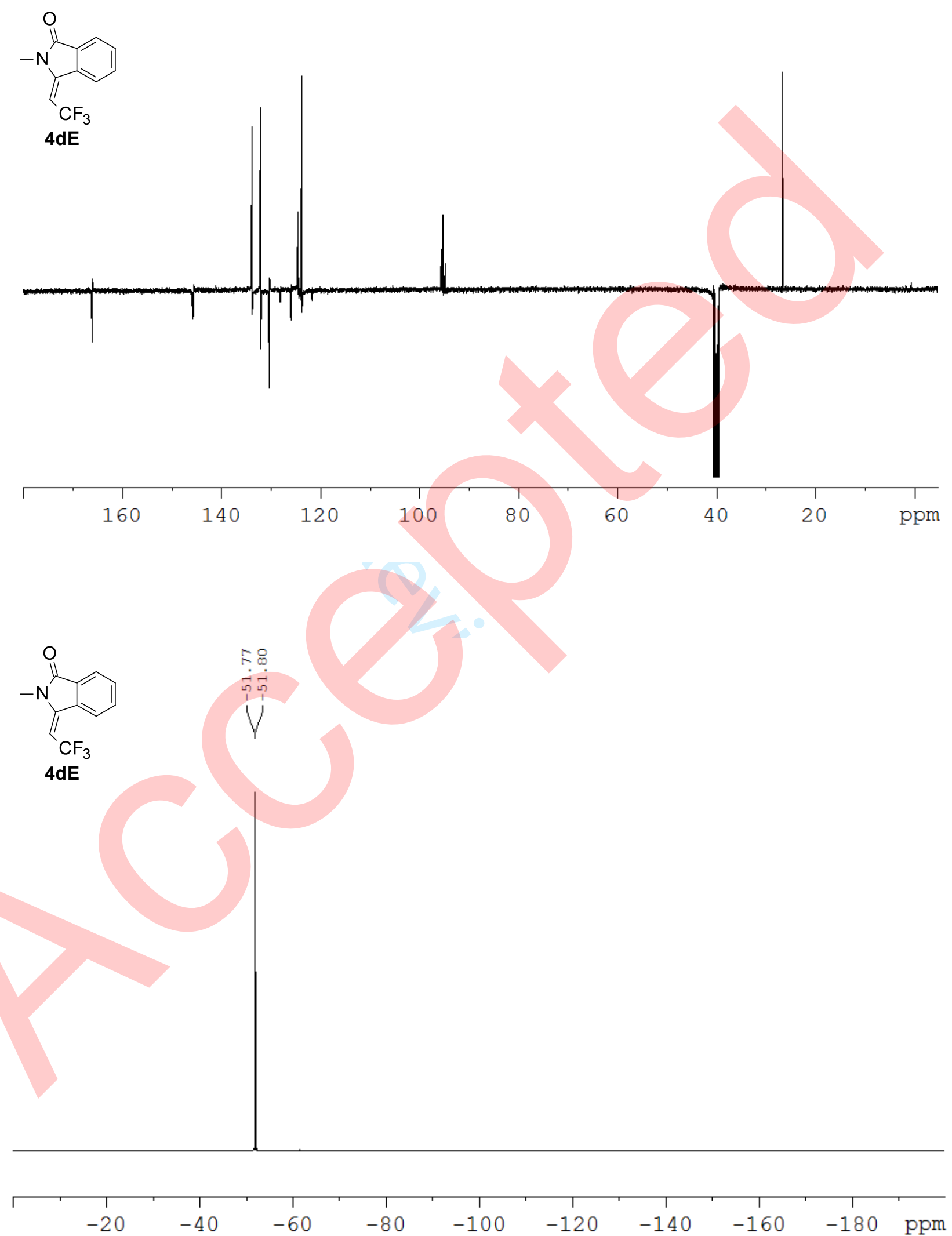

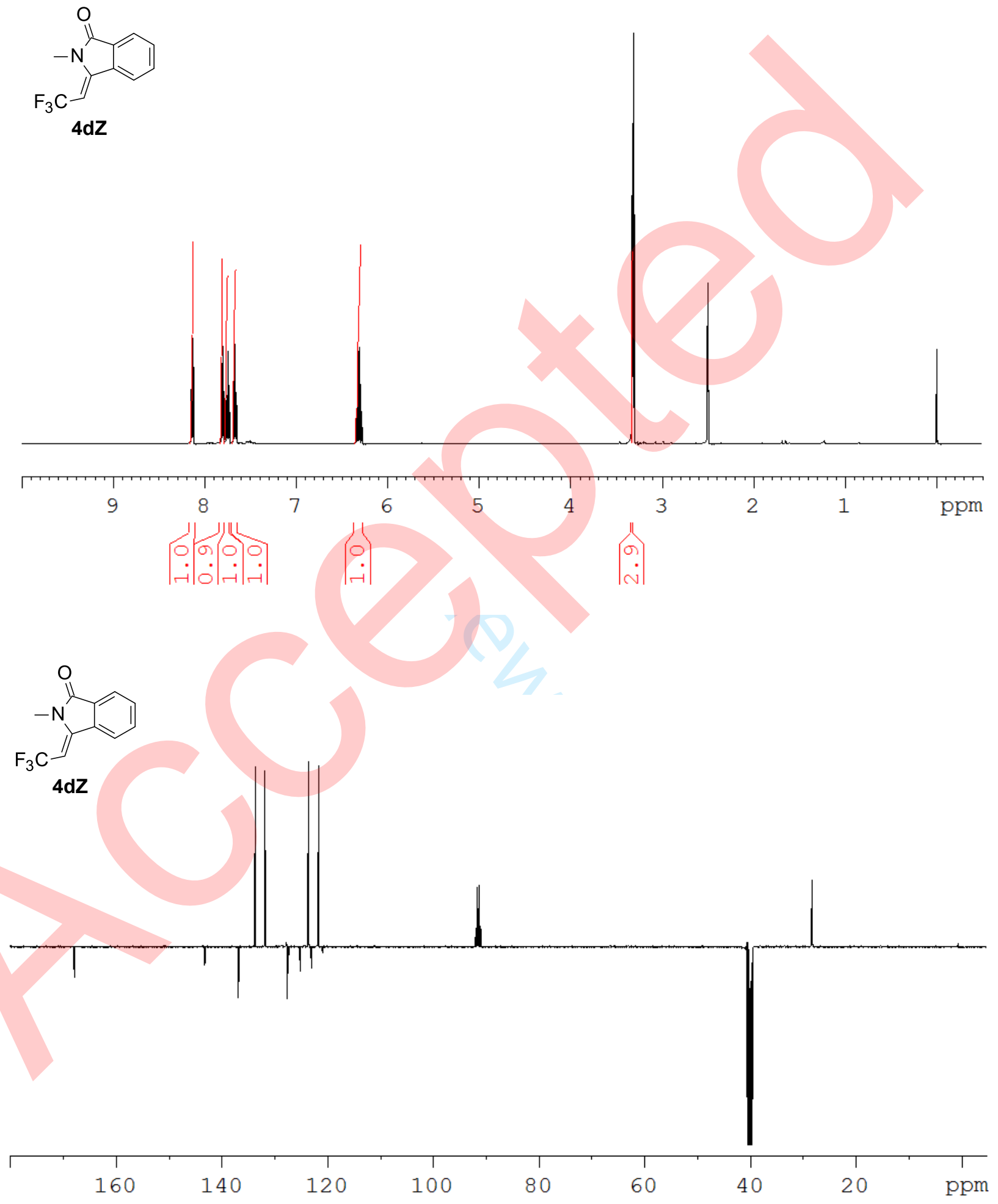


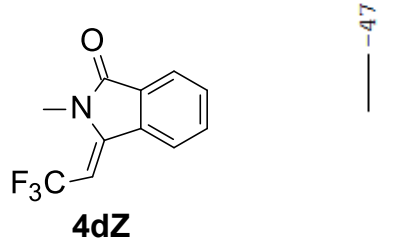
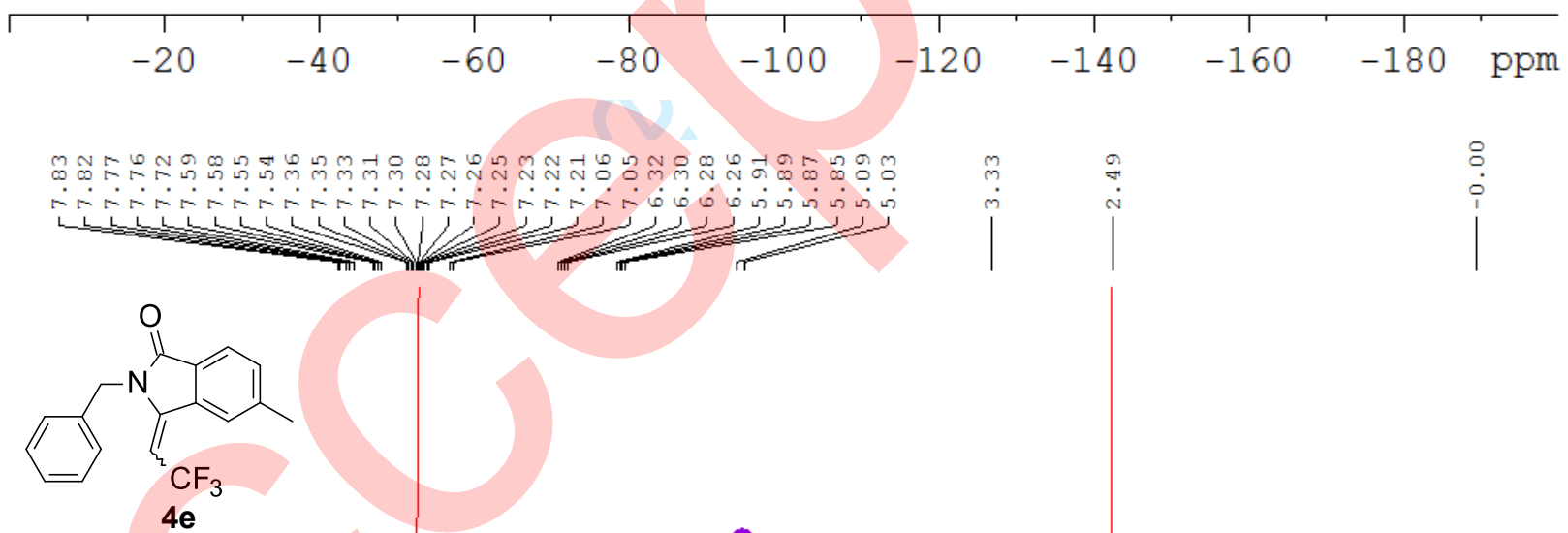

- 4eE

$4 \mathrm{eZ}$

47
4
5
6
7
8
9
0
1
2
5
4
5
6
57
58



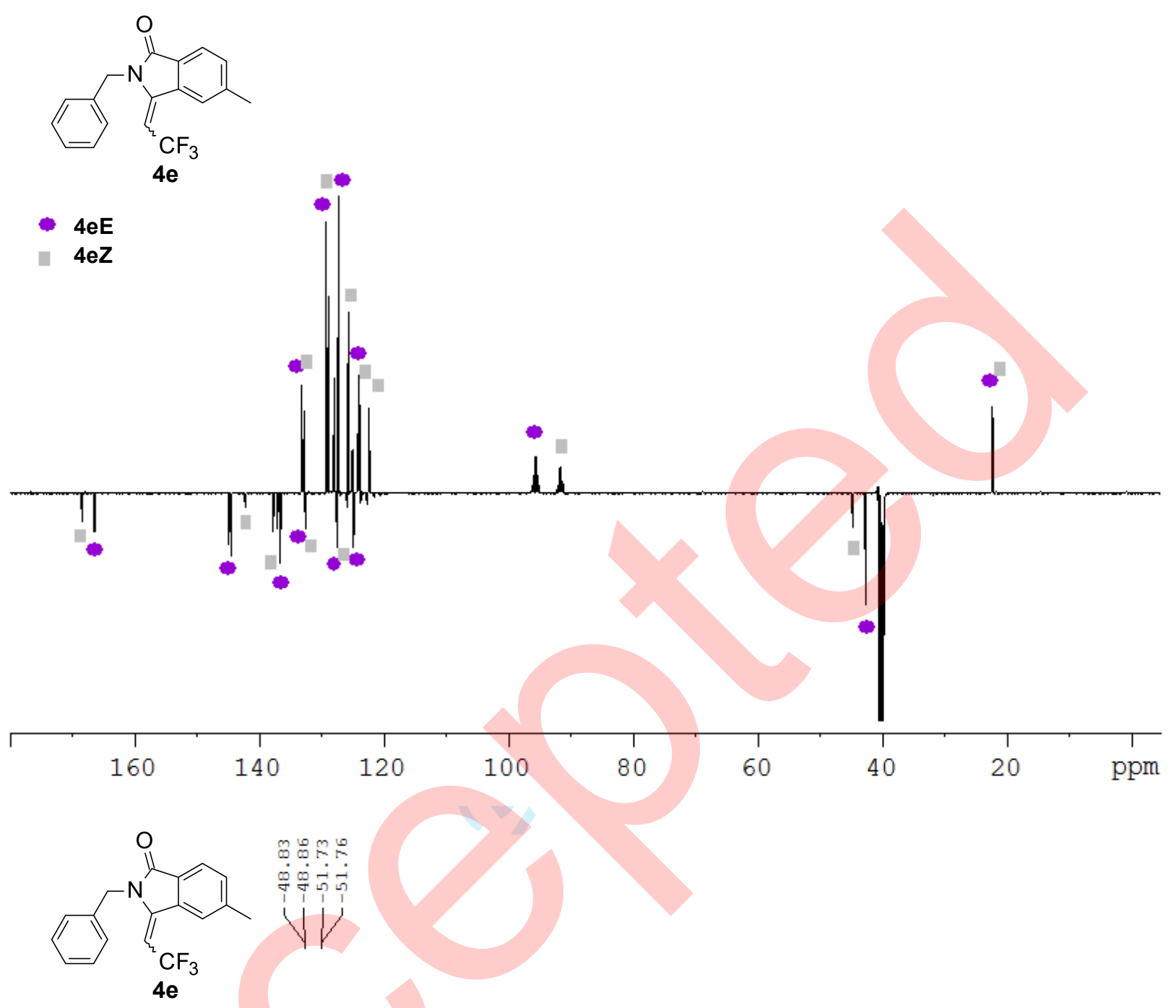

- 4eE

III $4 \mathrm{eZ}$

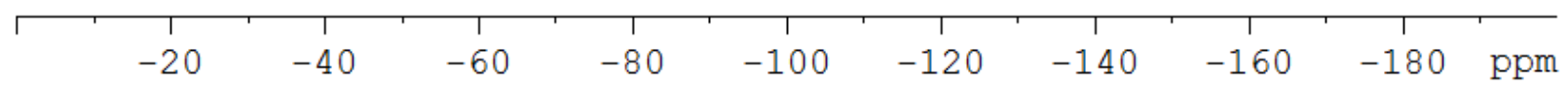




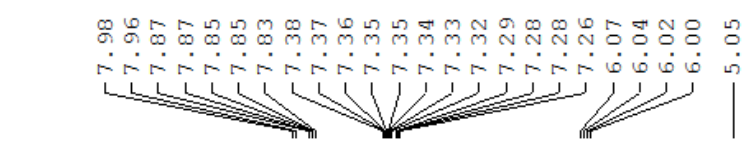<smiles>O=C1C(=O)N(Cc2ccccc2)/C(=C/C(F)(F)F)c2cc(Cl)ccc21</smiles>

1. $4 \mathrm{fZ}$

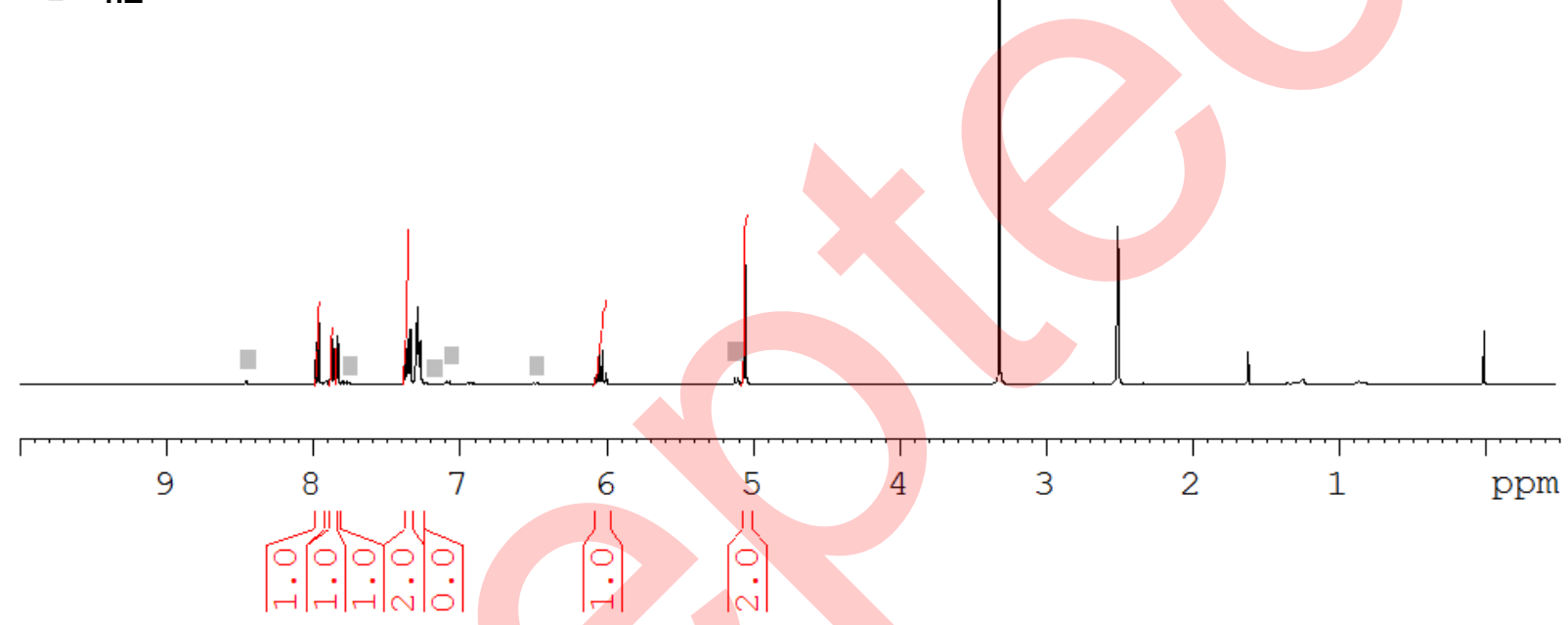<smiles>O=C1C(=O)N(Cc2ccccc2)/C(=C/C(F)(F)F)c2cc(Cl)ccc21</smiles>

$4 \mathrm{fE}$ 
<smiles>CC(F)(F)/C=C1\c2cc(Cl)ccc2C(=O)N1Cc1ccccc1</smiles>

III $4 \mathrm{fZ}$
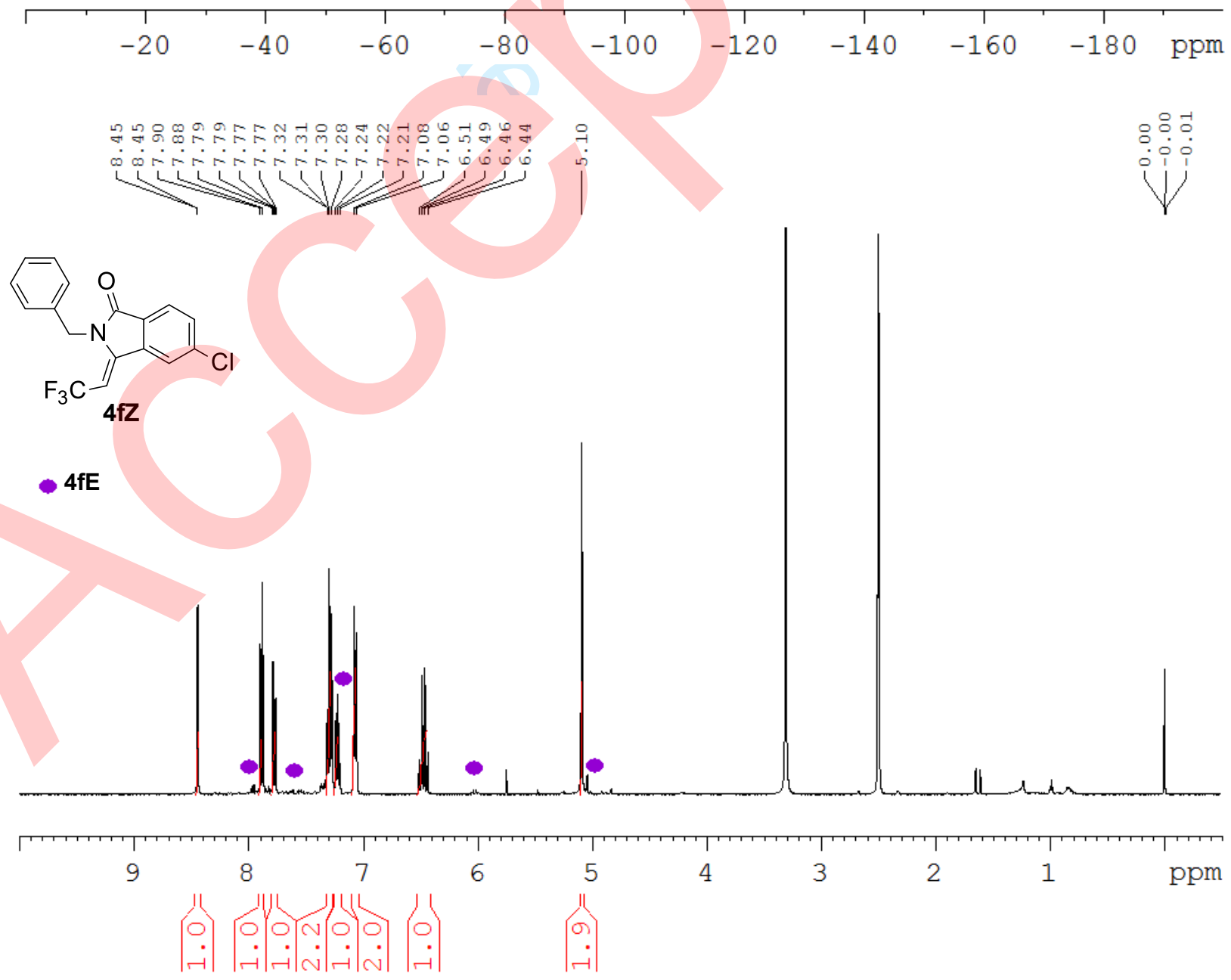

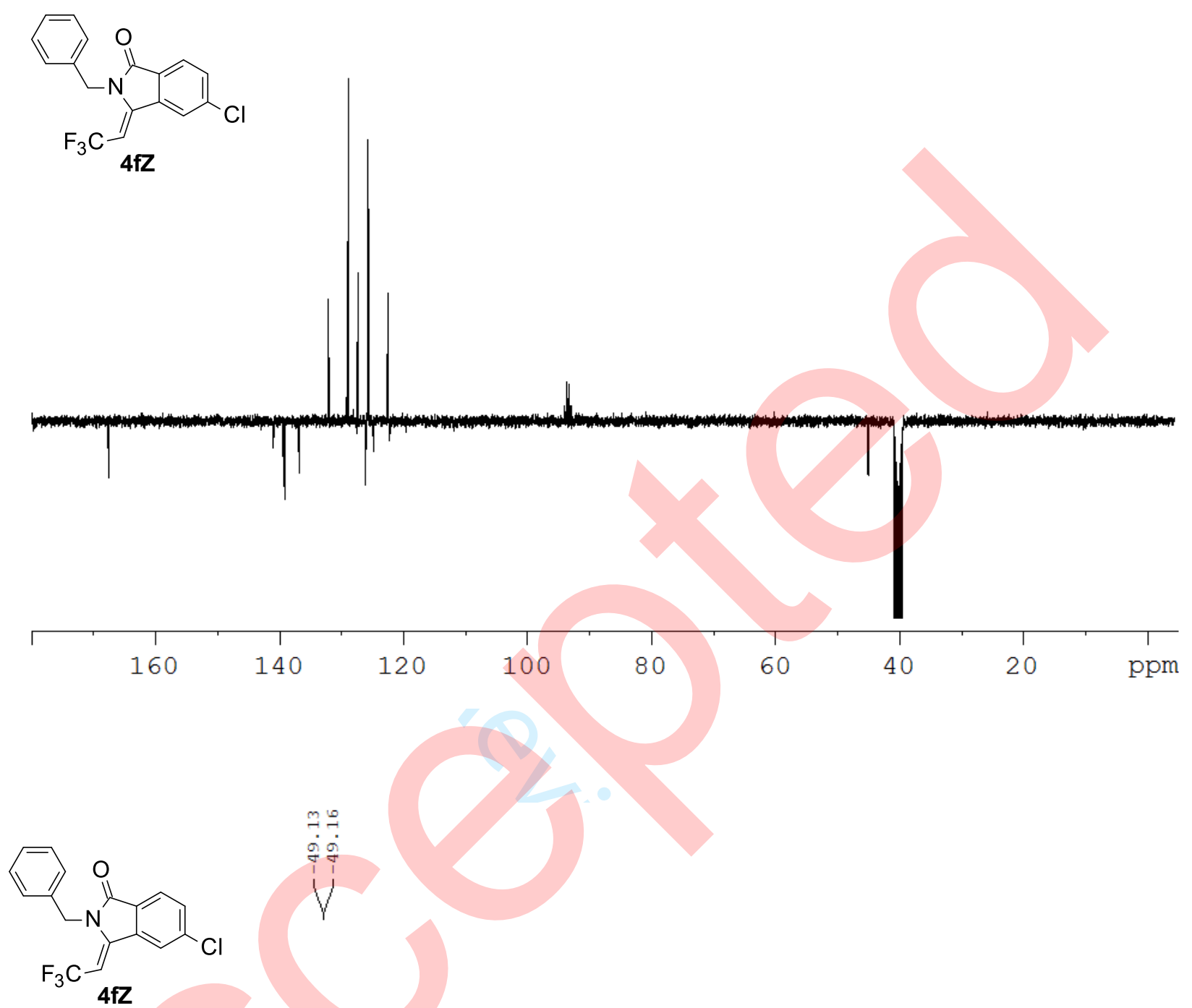

- 4fE
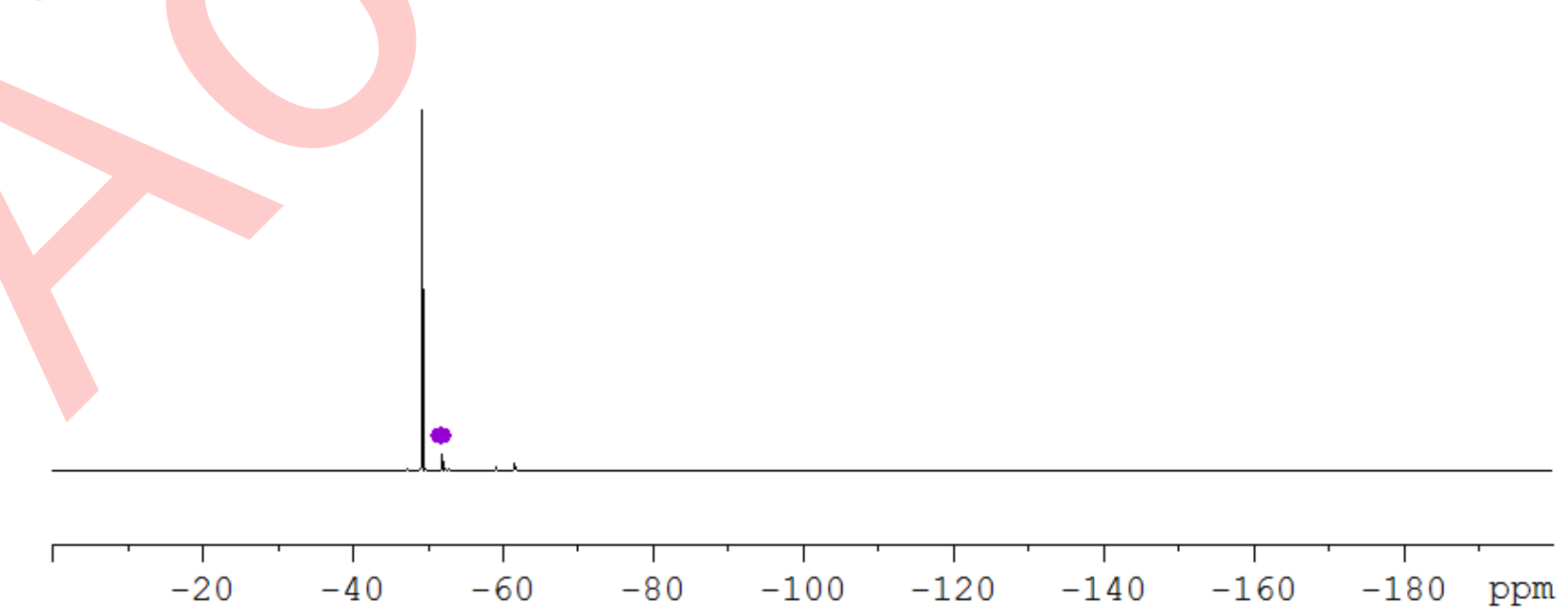
<smiles>O=C1c2ccc(F)cc2/C(=C\C(F)(F)C(F)(F)F)N1Cc1ccccc1</smiles>

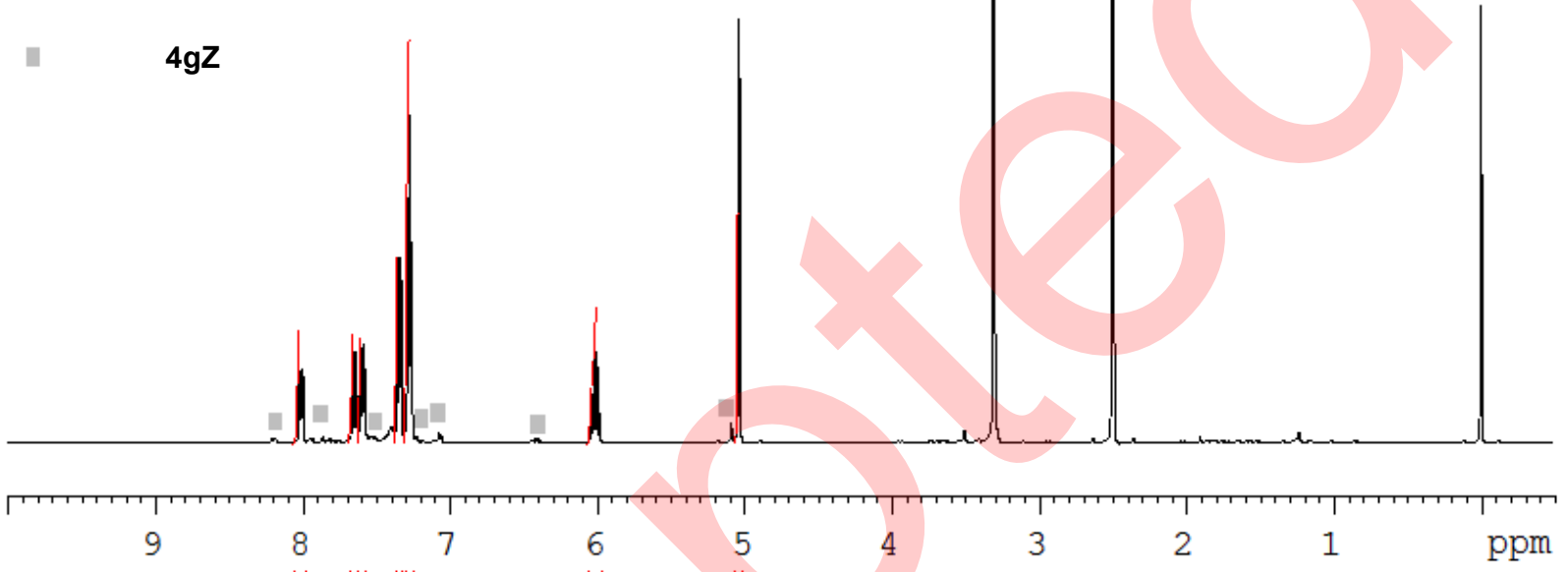

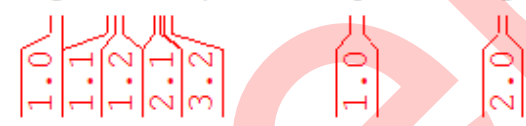<smiles>CC(C)(C)/C=C1\c2cc(F)ccc2C(=O)C(=O)N1Cc1ccccc1</smiles>

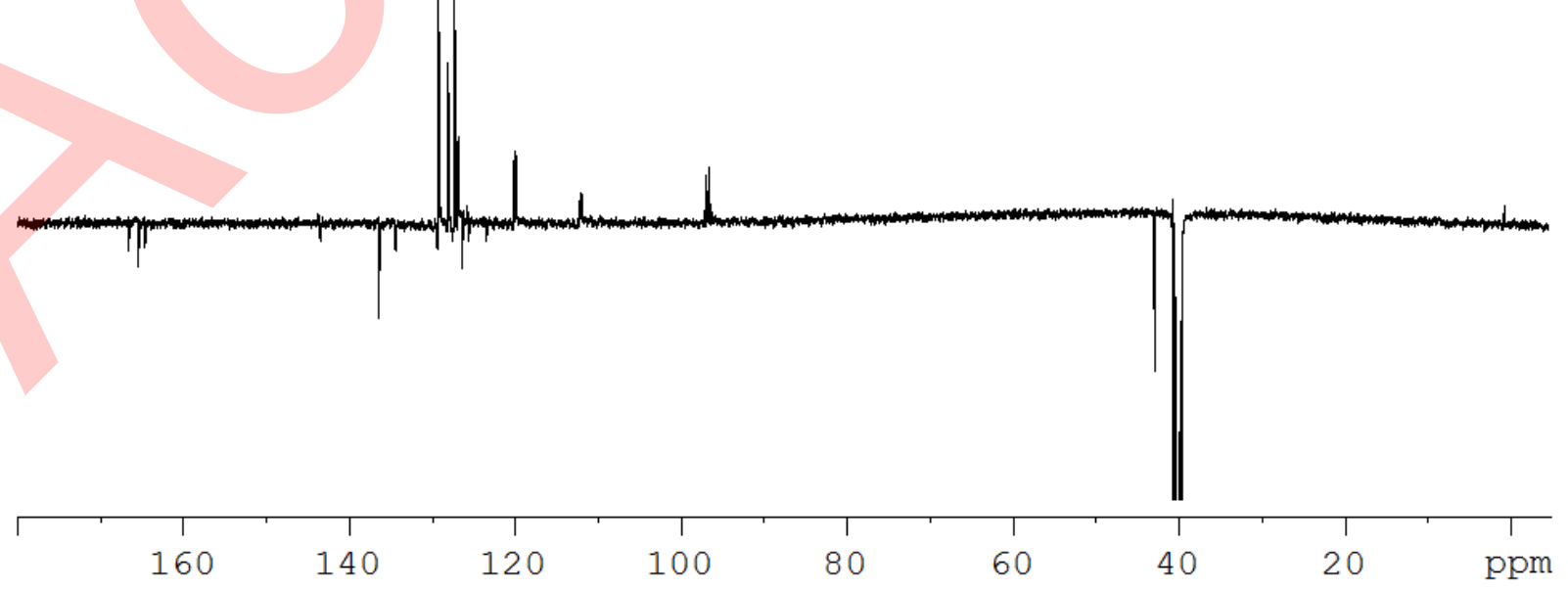



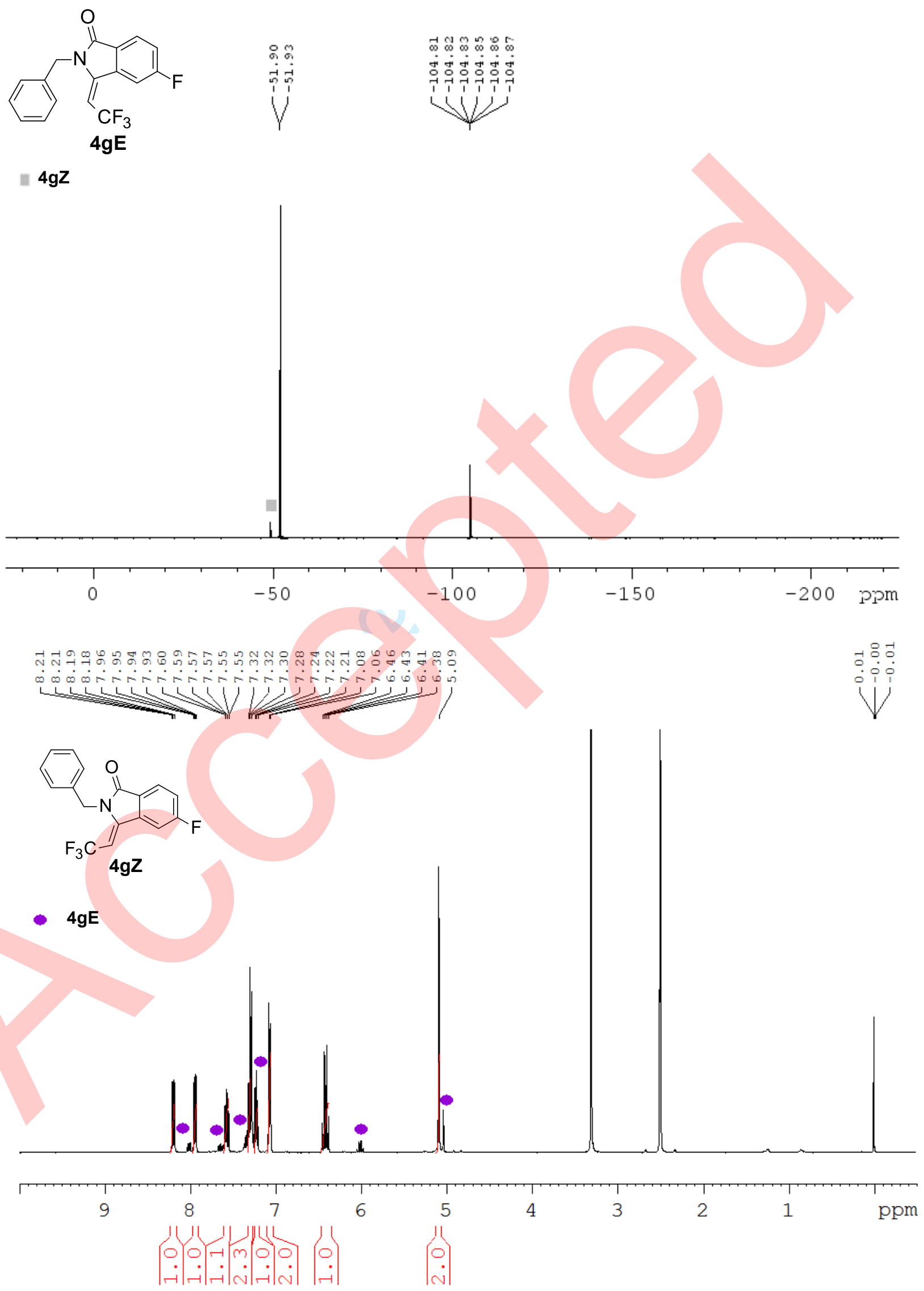

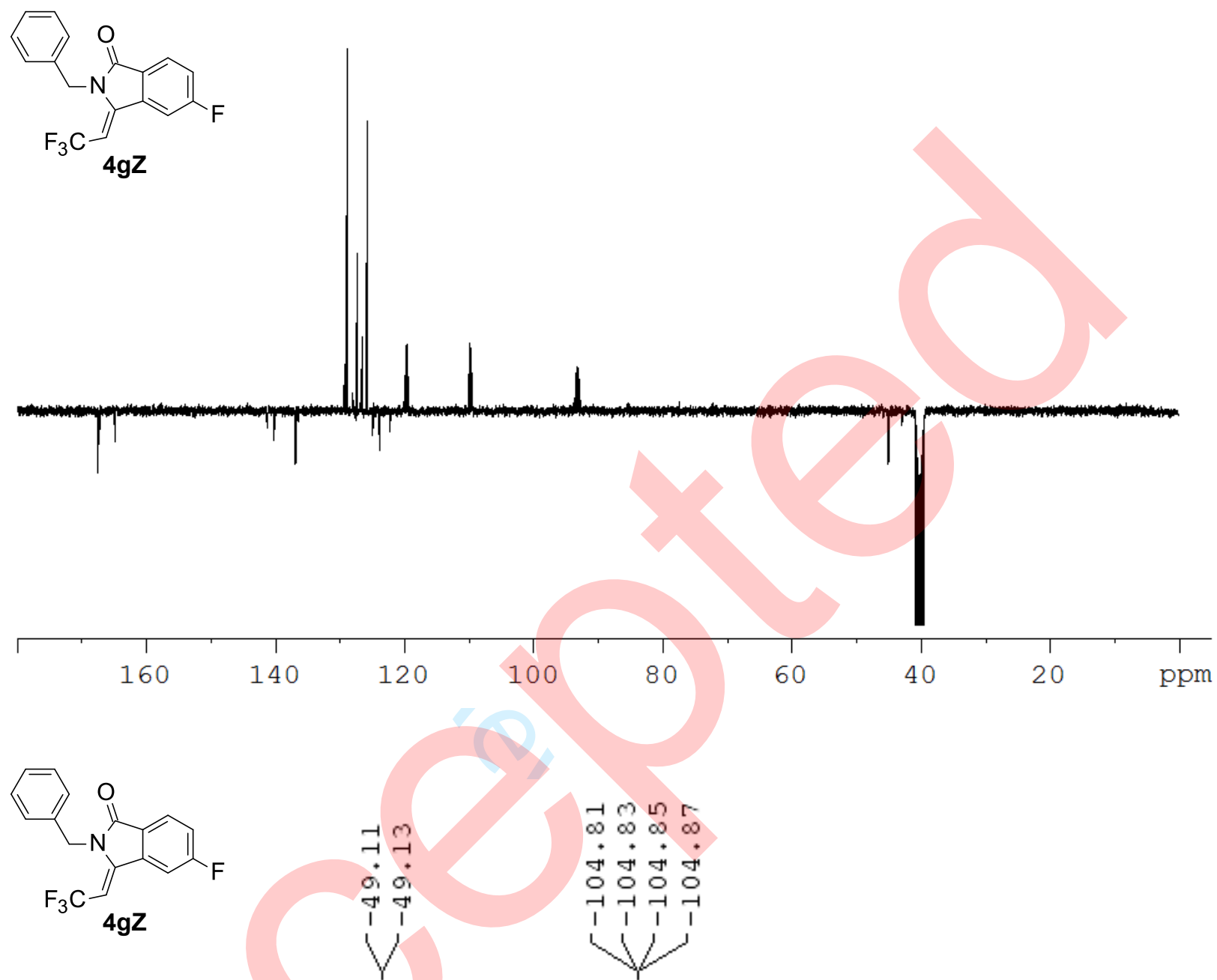

- 4aE 

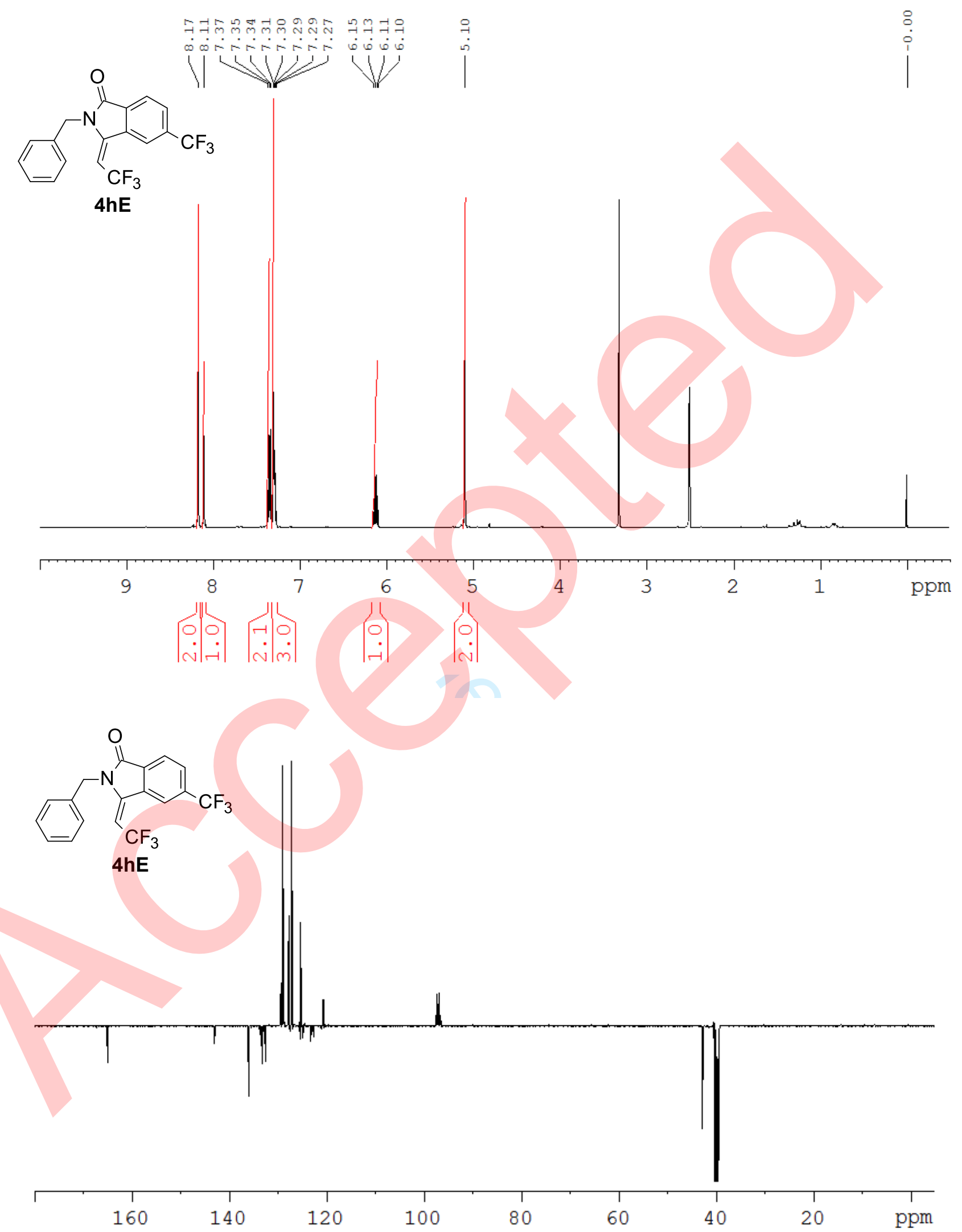


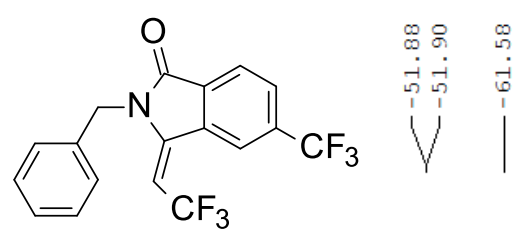

4hE
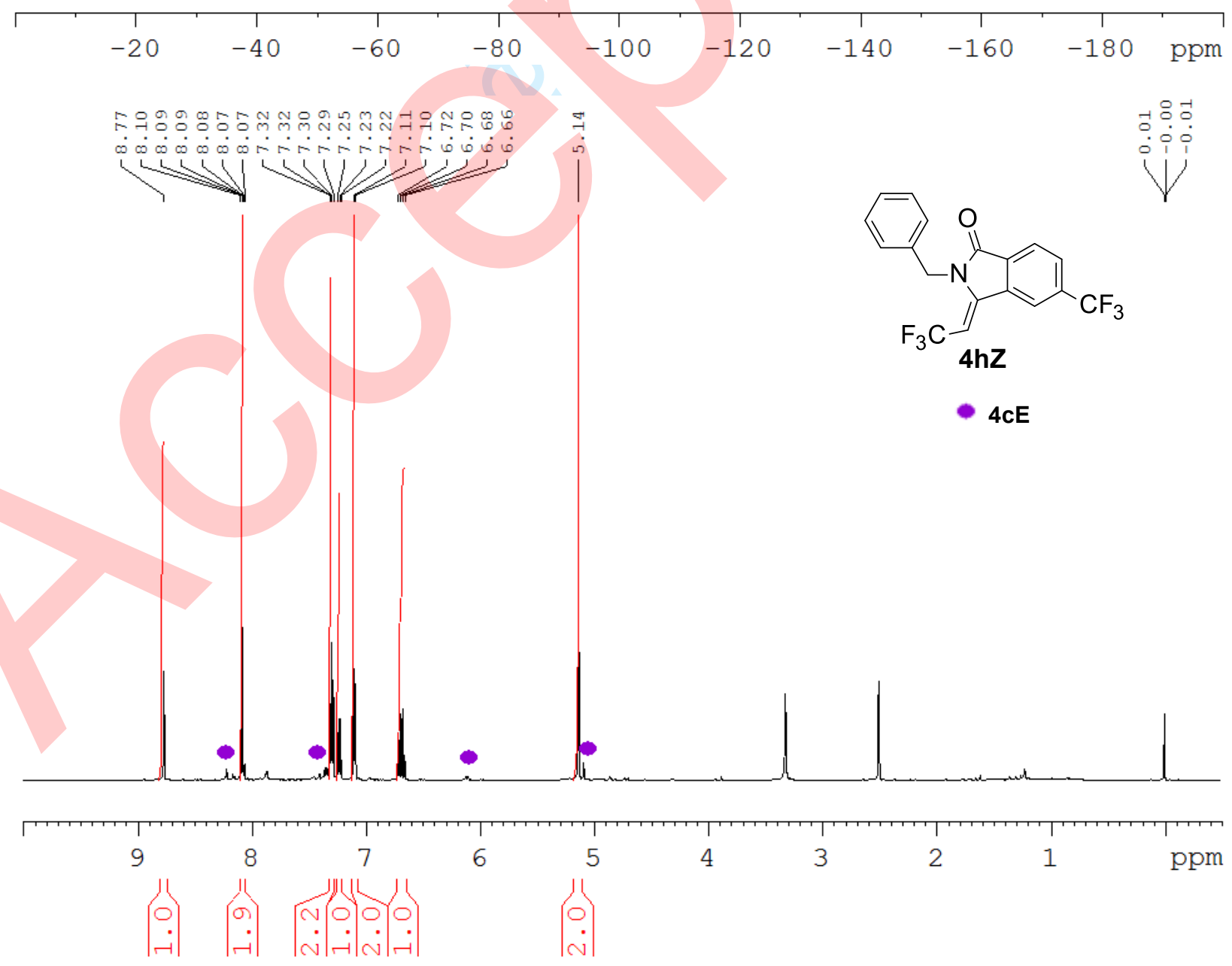

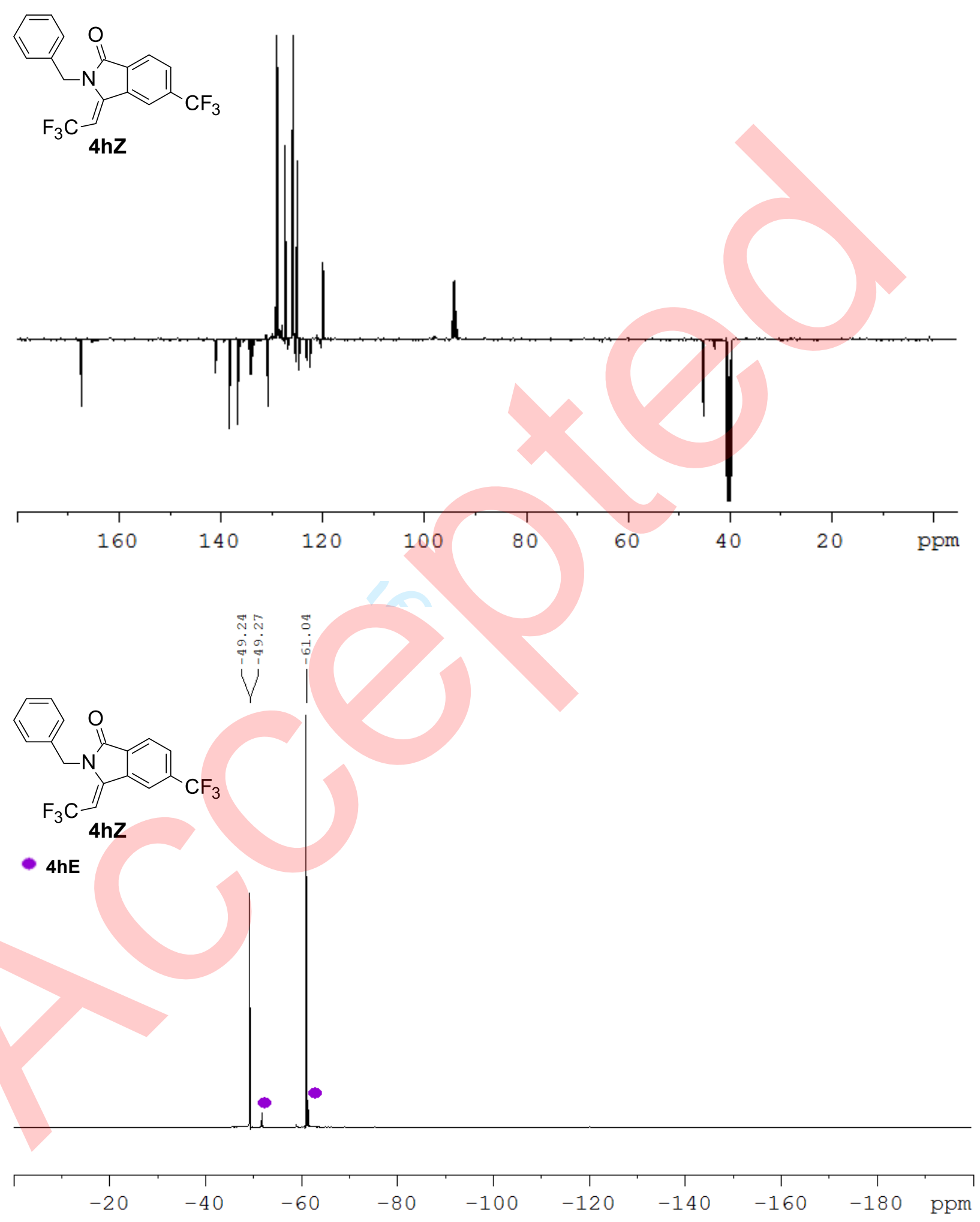


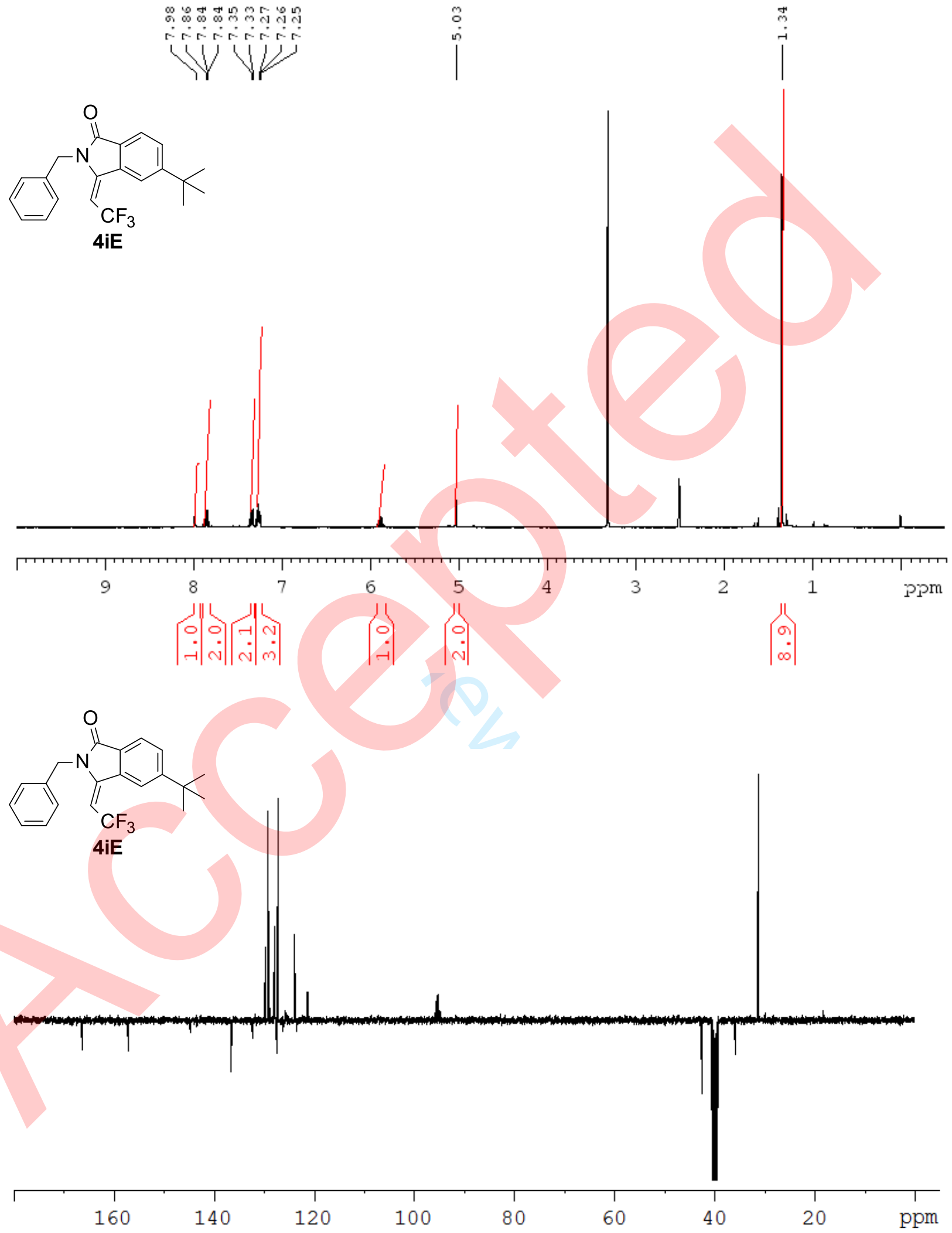




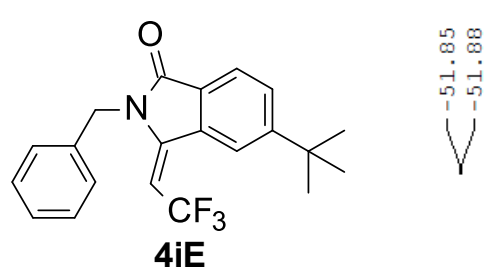

$\mathbf{4} \mathbf{c Z}$
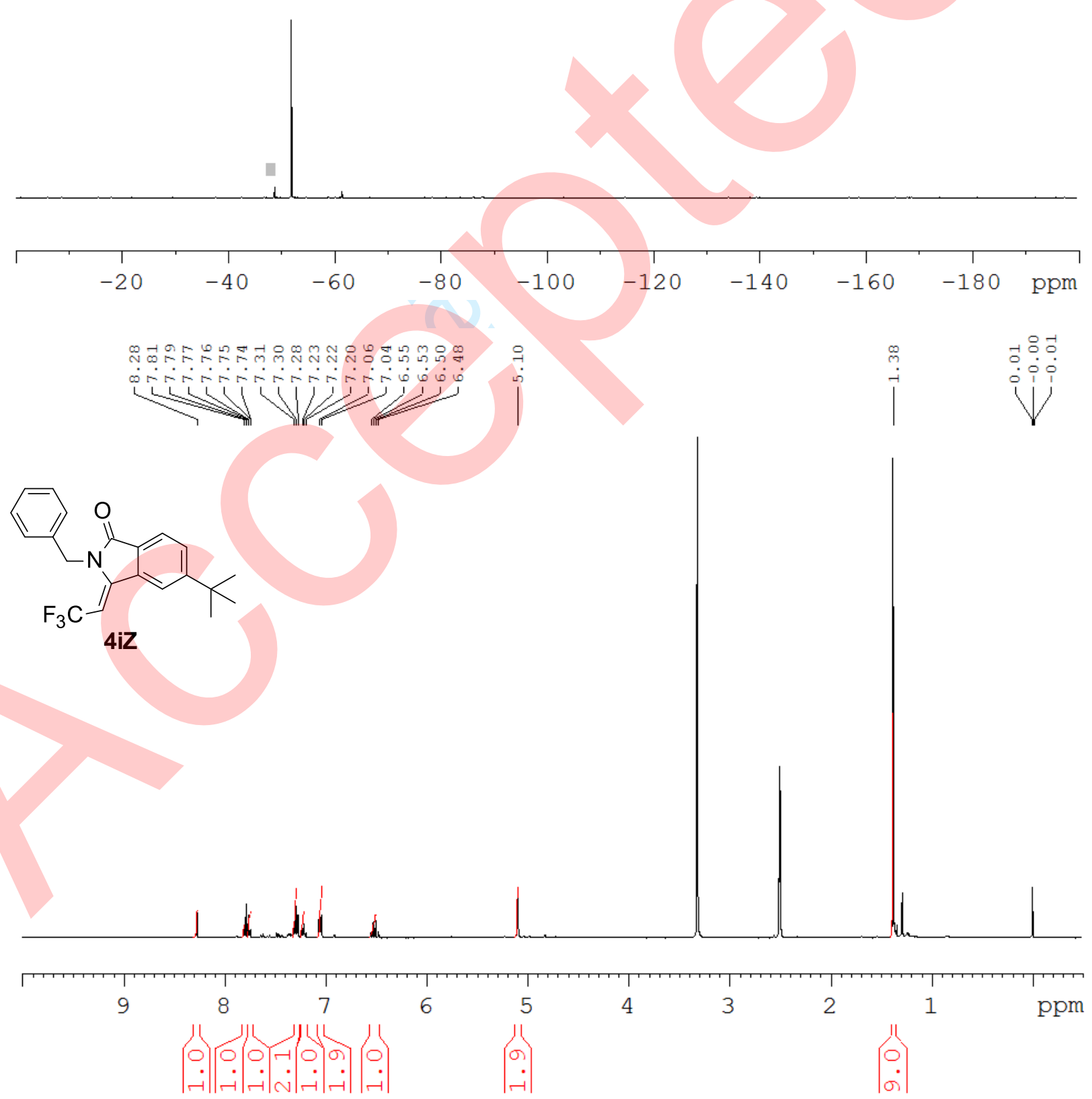

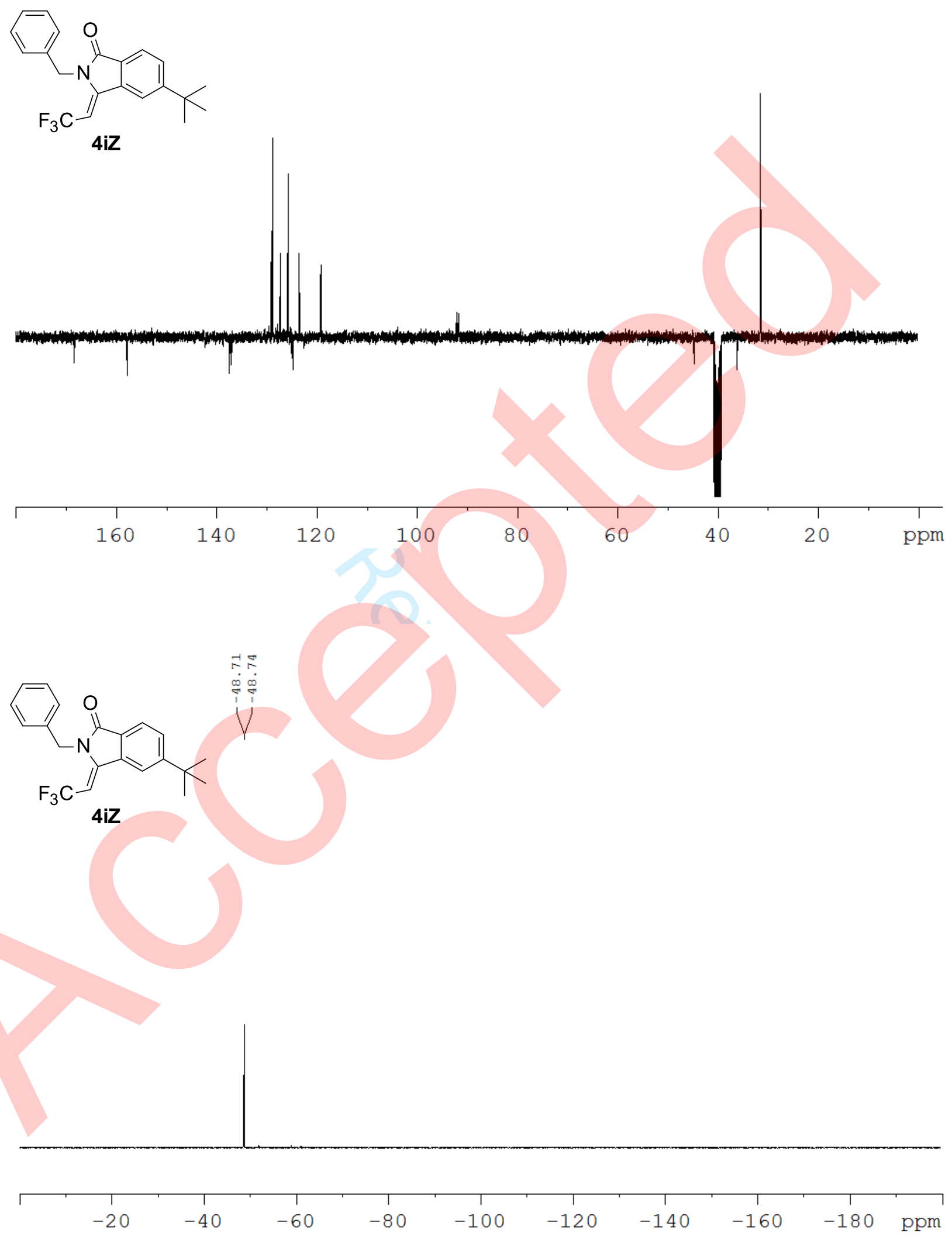
<smiles>O=C1c2ccc([N+](=O)[O-])cc2/C(=C\C(F)(F)F)N1Cc1ccccc1</smiles>

4j

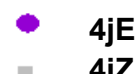

- $4 j Z$
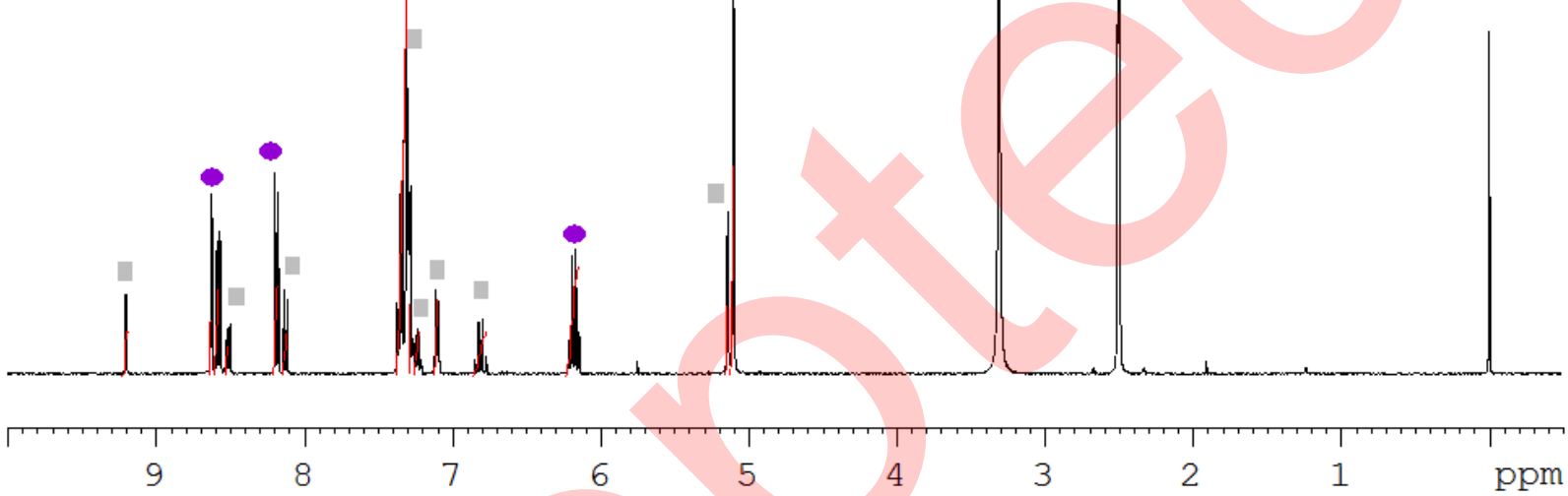

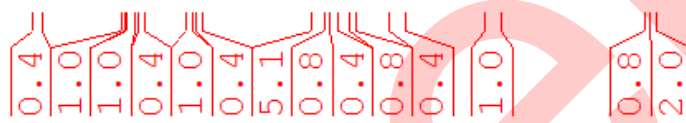

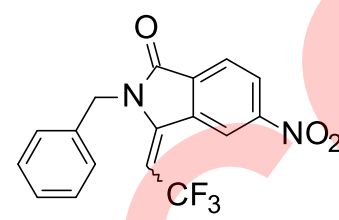

$4 \mathbf{j}$

- $4 \mathrm{bE}$ $4 b Z$

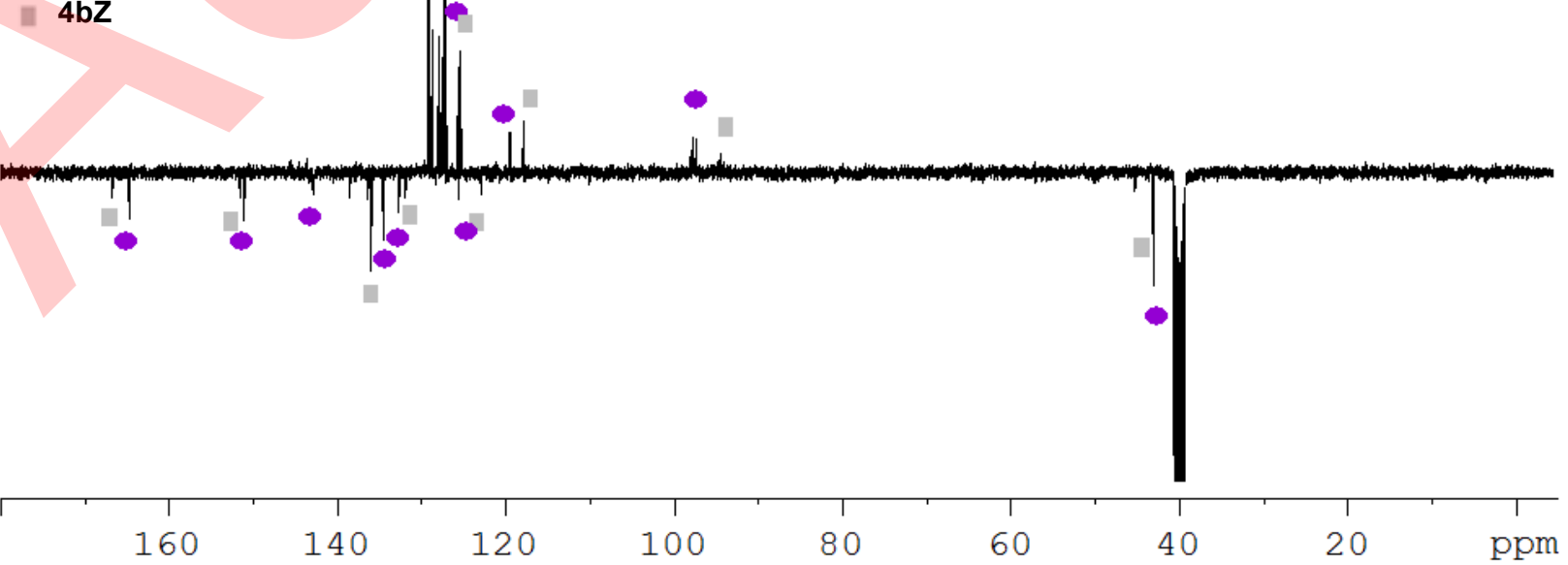




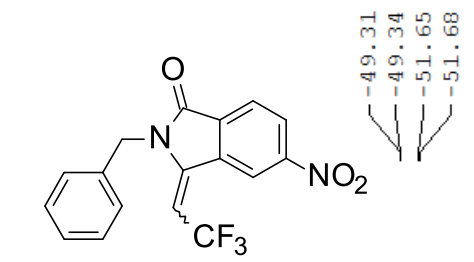

$4 \mathbf{j}$
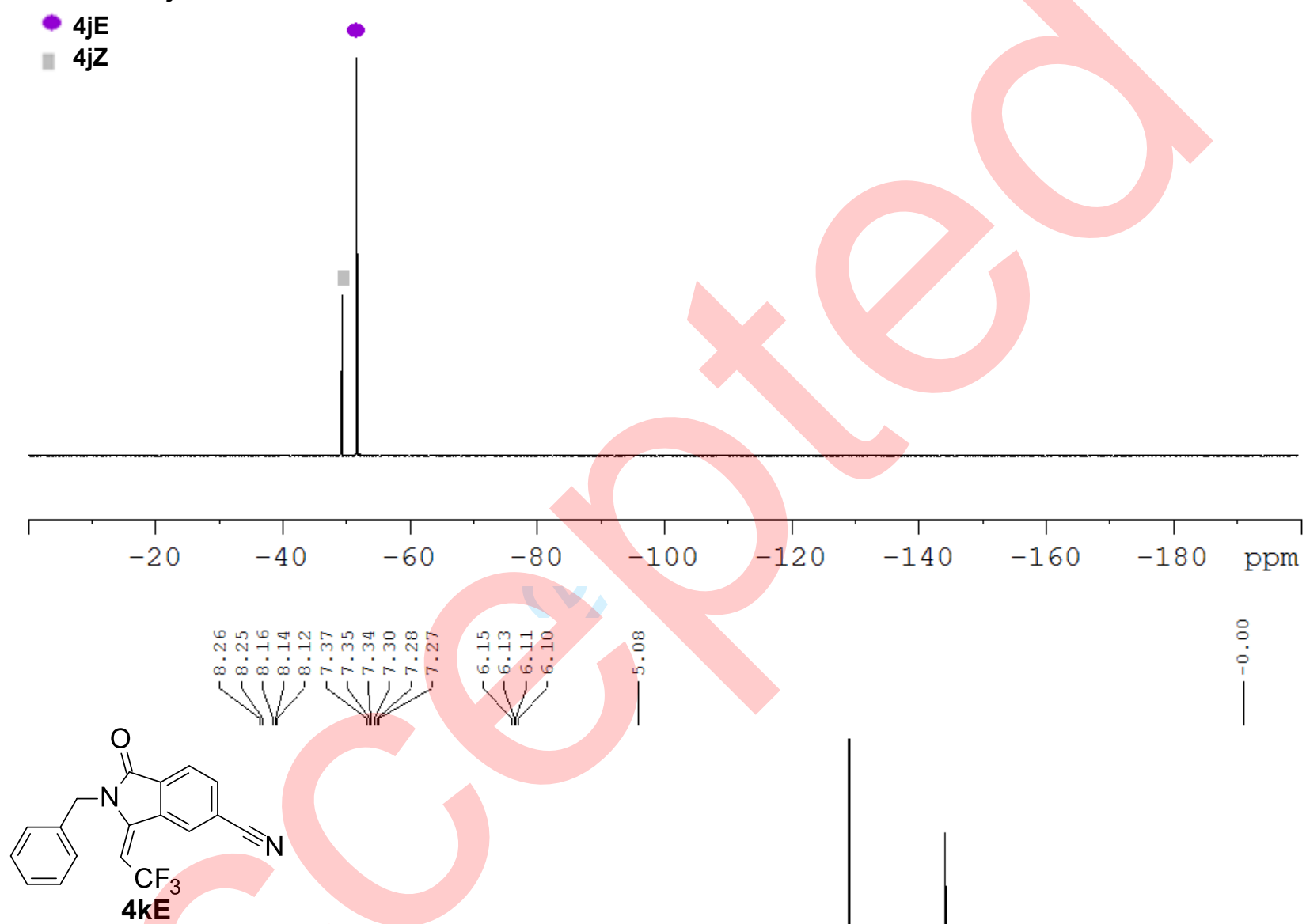

- $4 k Z$

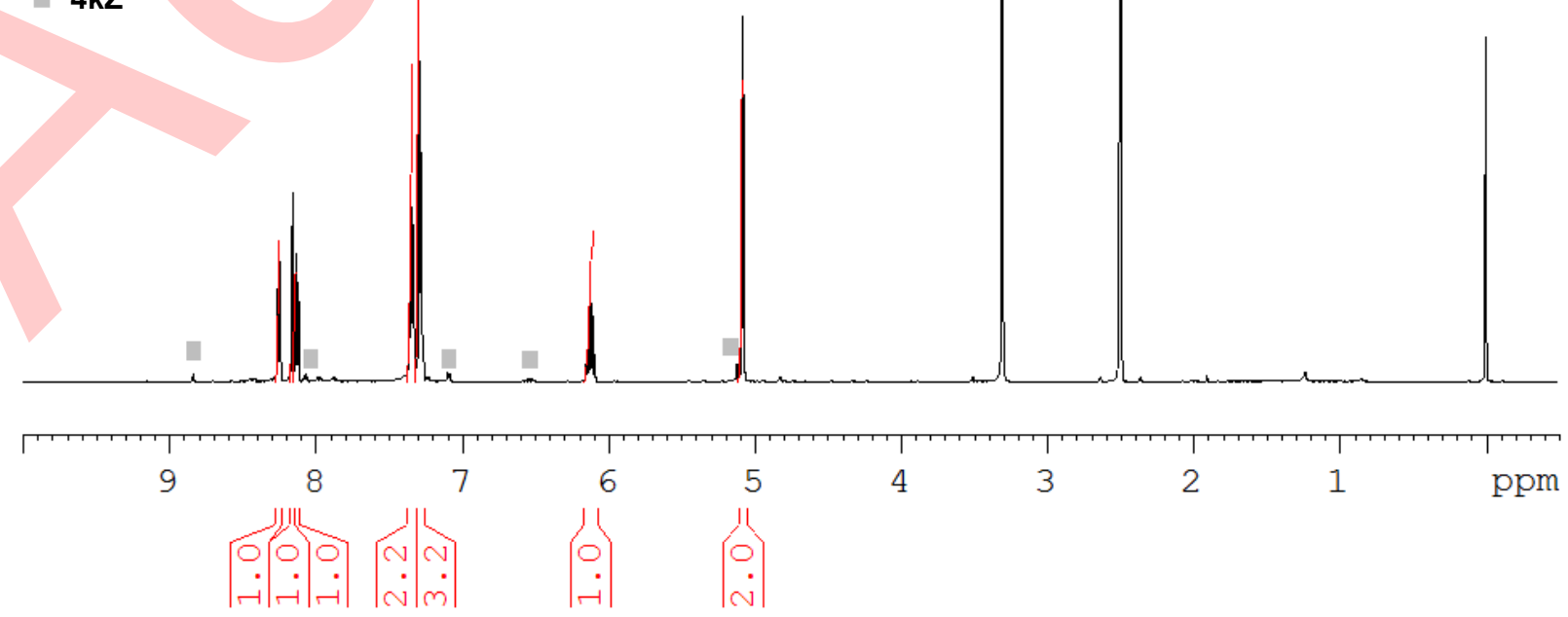



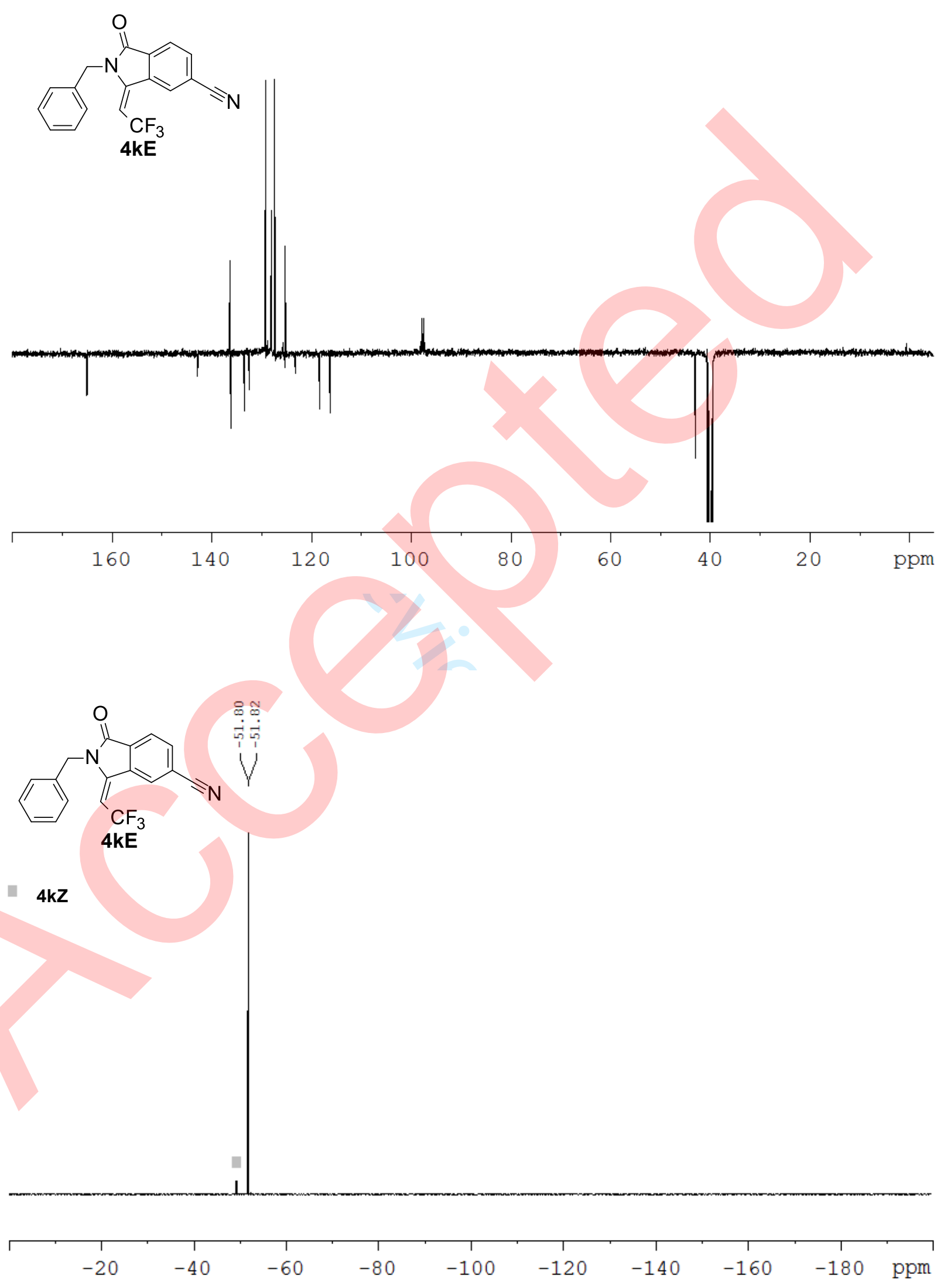

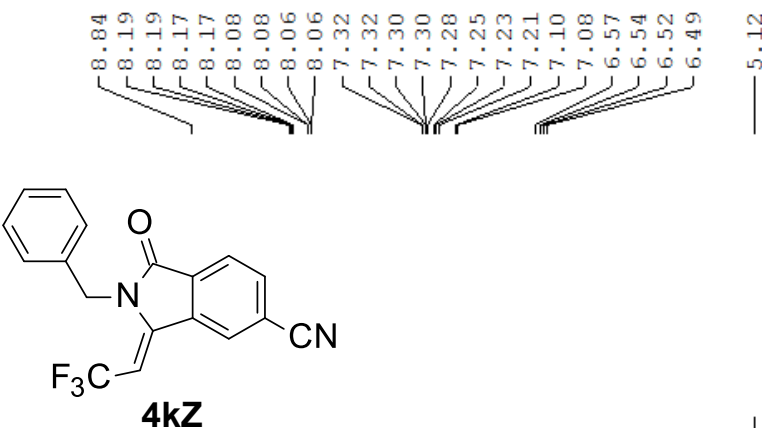

- 4kE
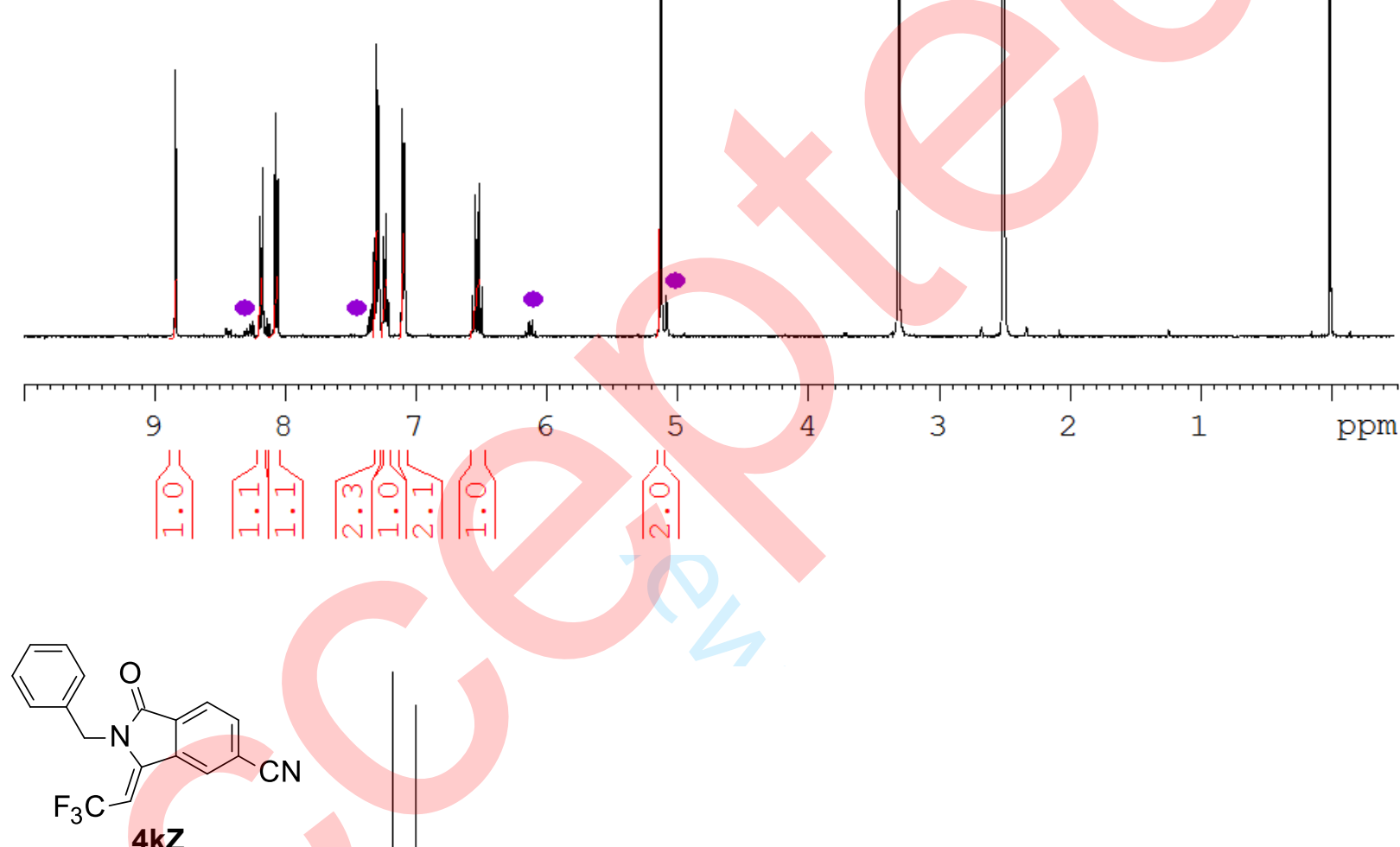

\section{$4 k Z$}

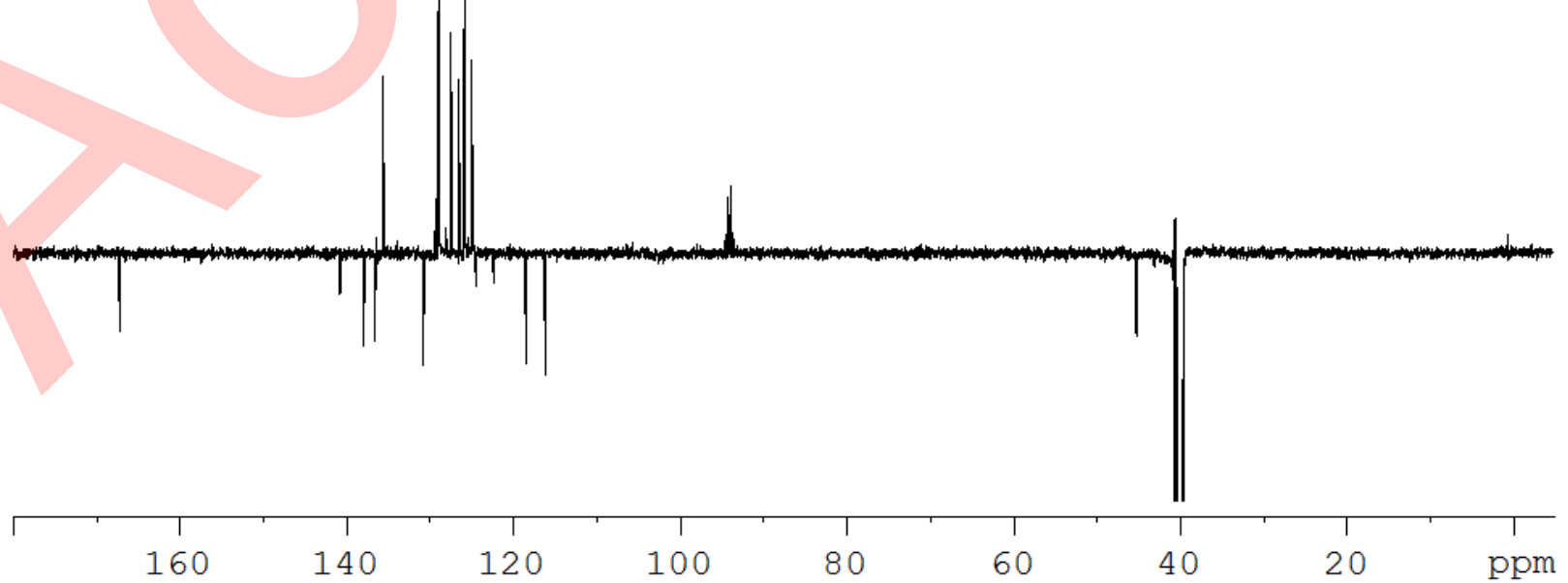



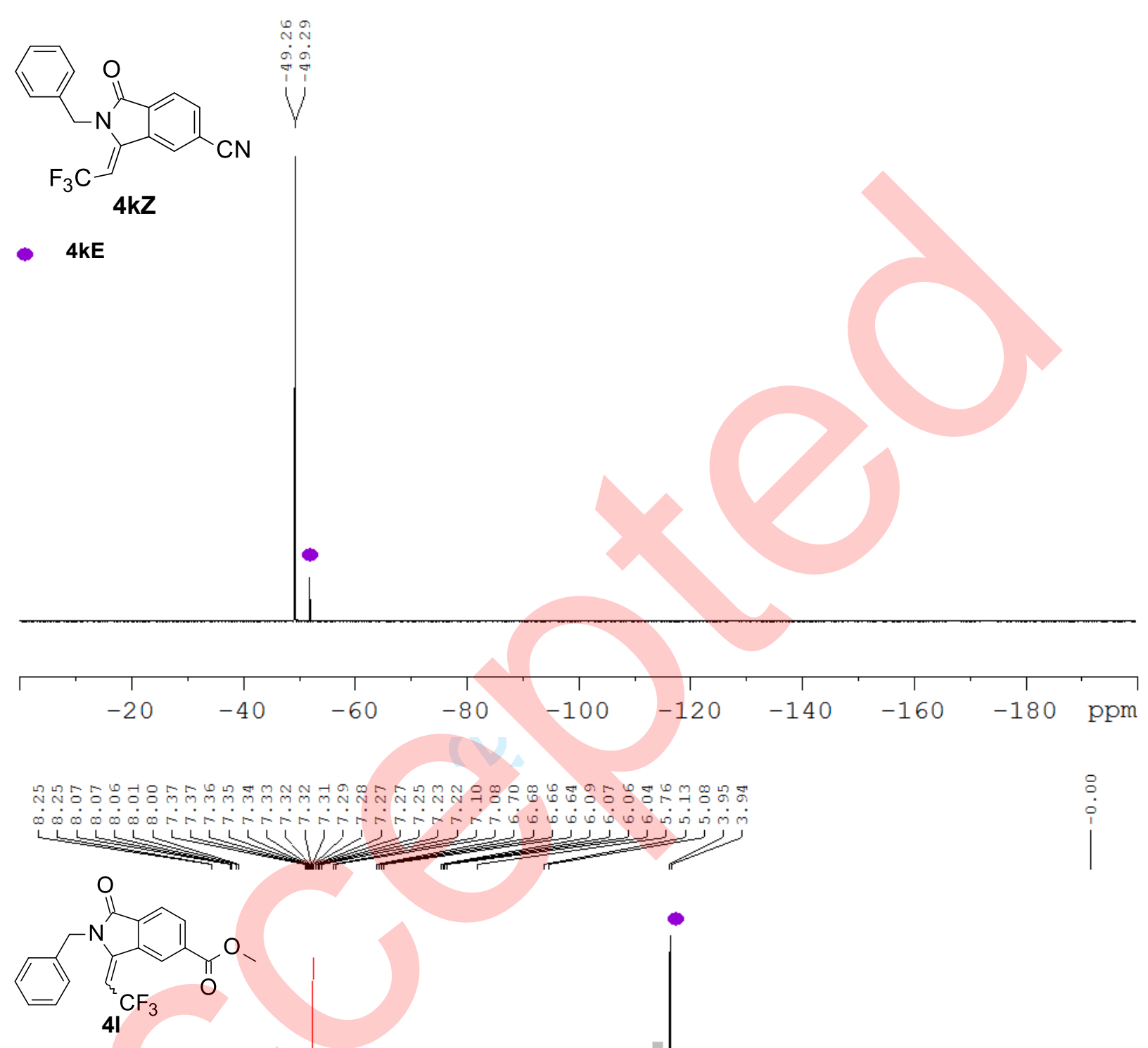

4IE
$-\quad 4 I Z$

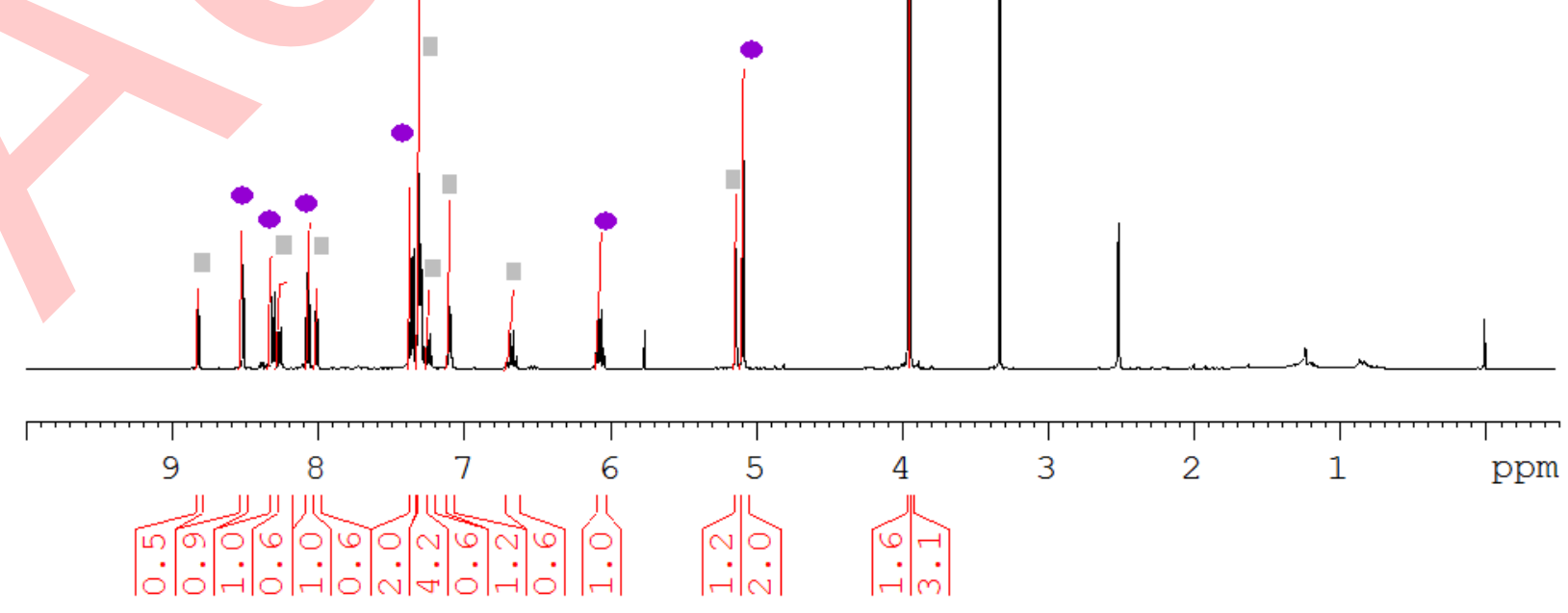



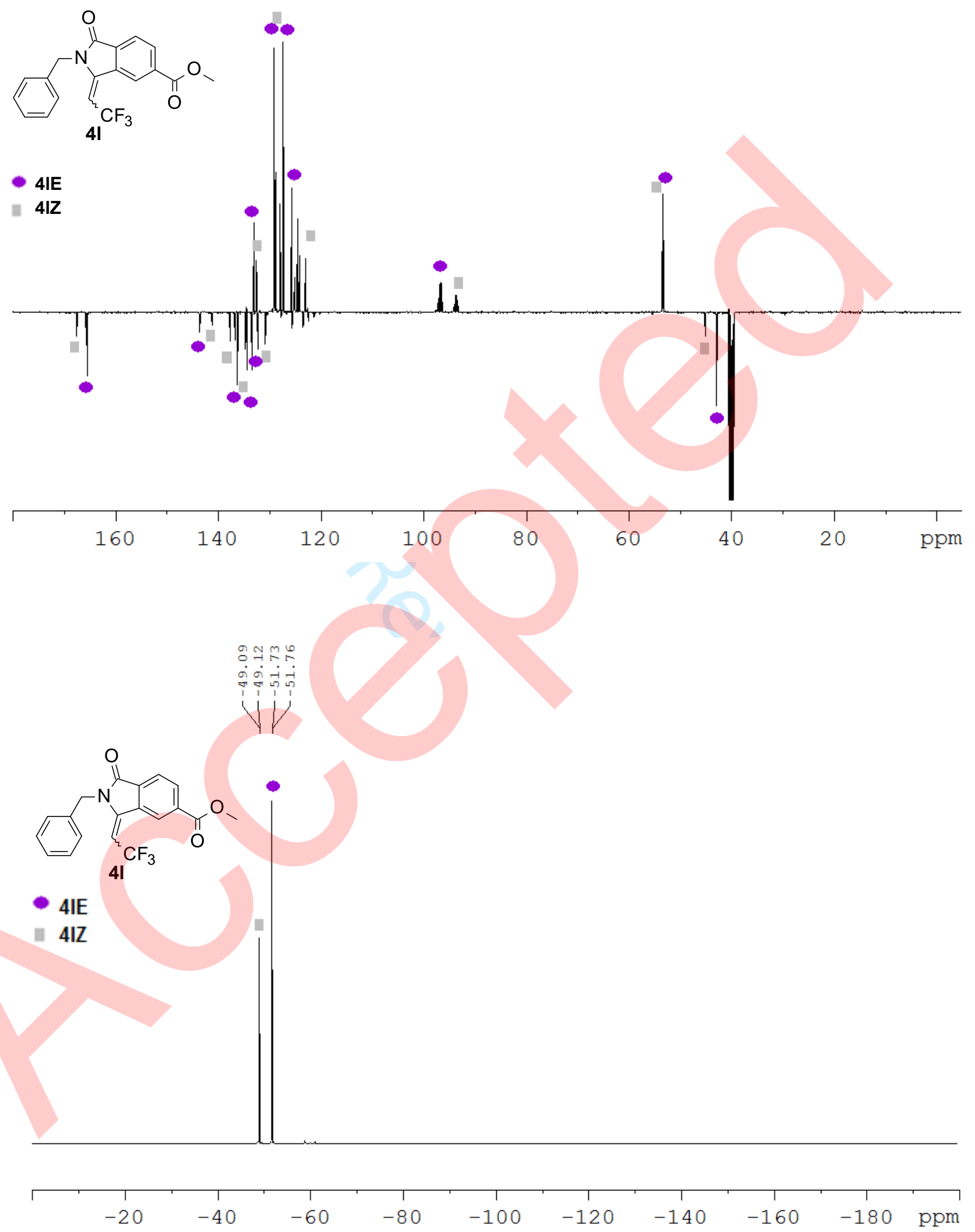


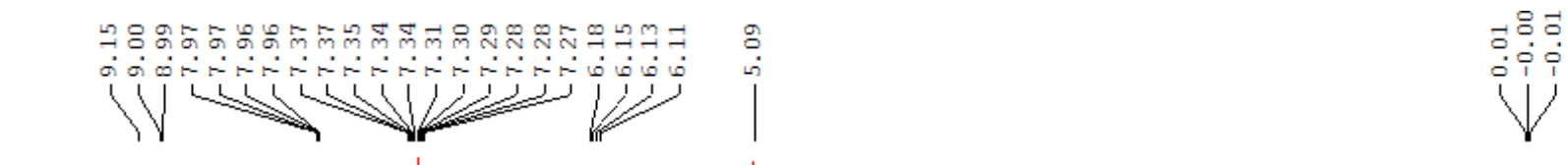<smiles>O=C1c2ccncc2C(=CC(F)(F)F)N1Cc1ccccc1</smiles>

$4 \mathrm{mE}$

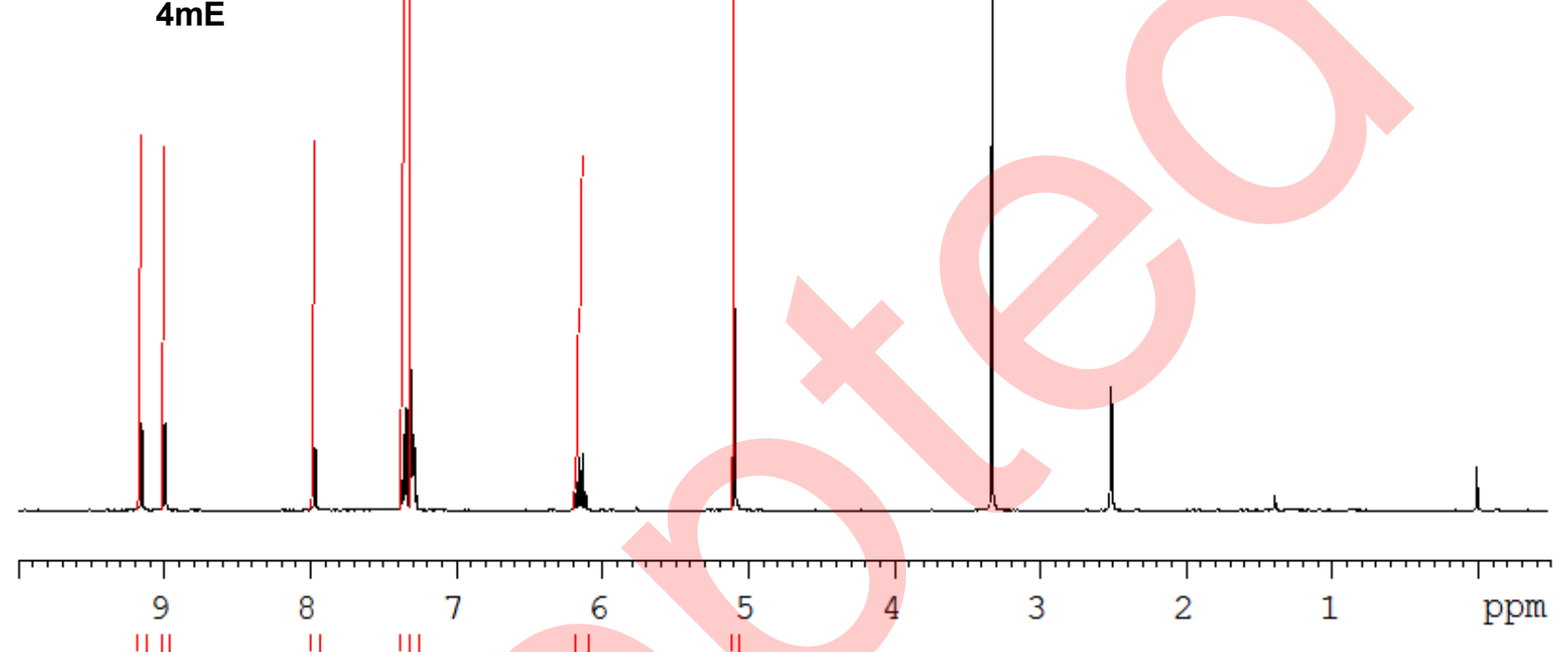

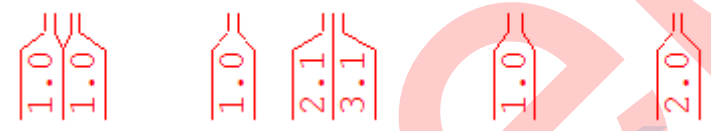<smiles>O=C1C(=CC(F)(F)F)/C(=C/C(F)(F)F)c2cnccc21</smiles>
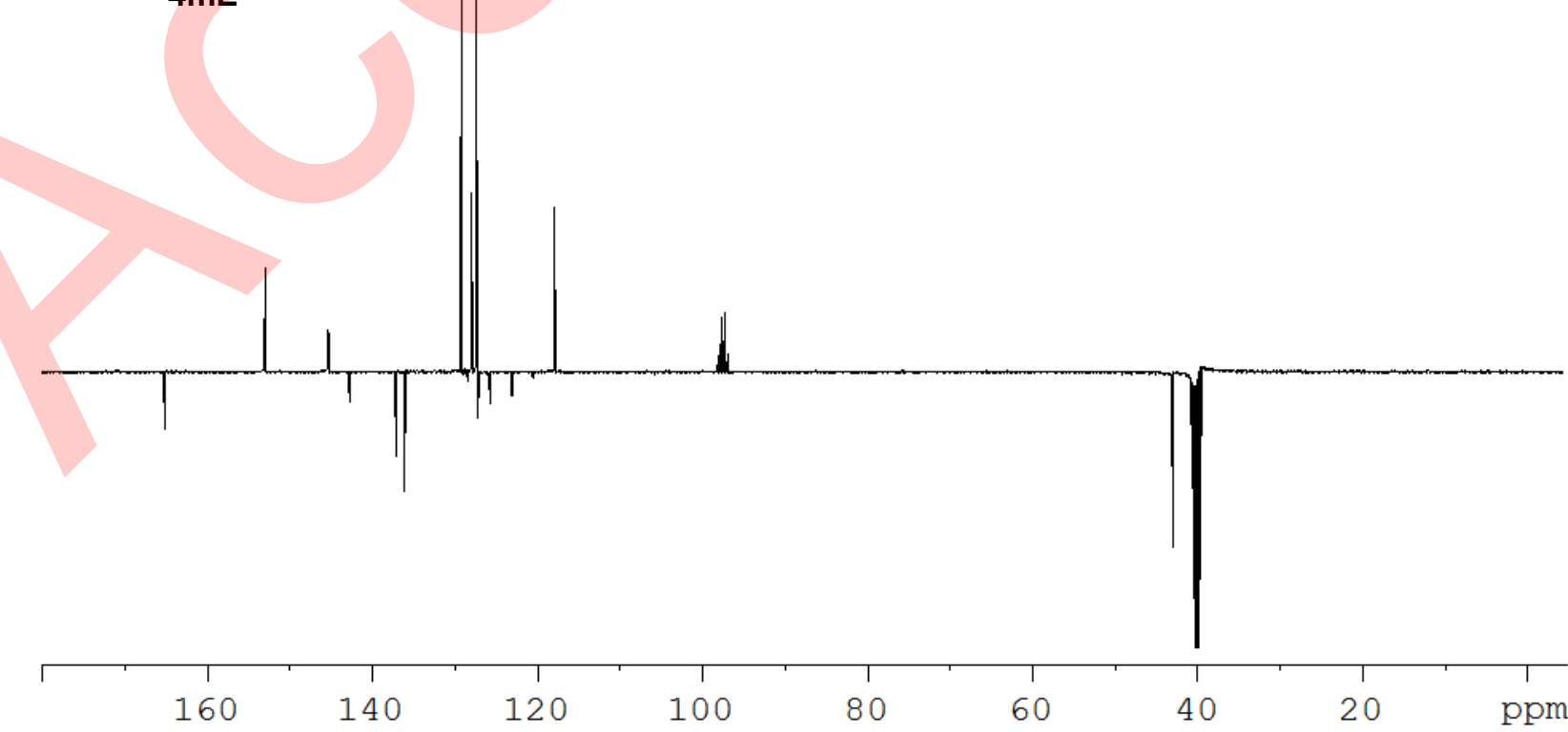


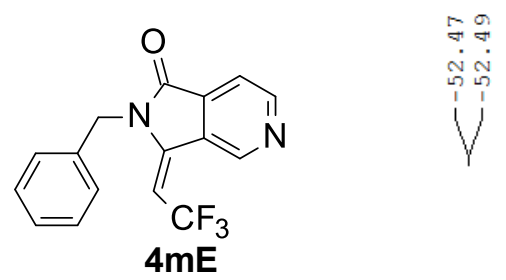

$4 \mathrm{mE}$
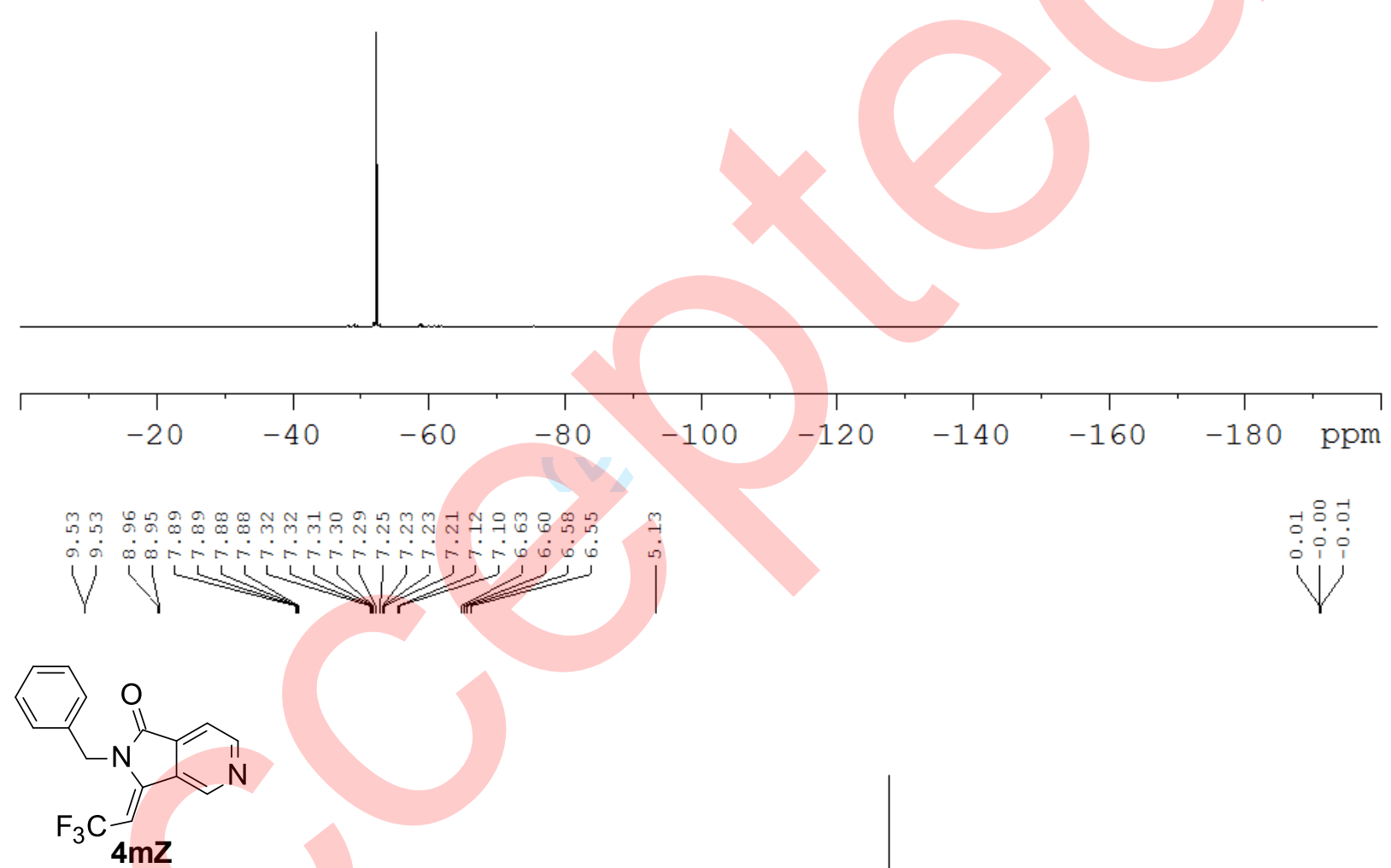

41

42

43 

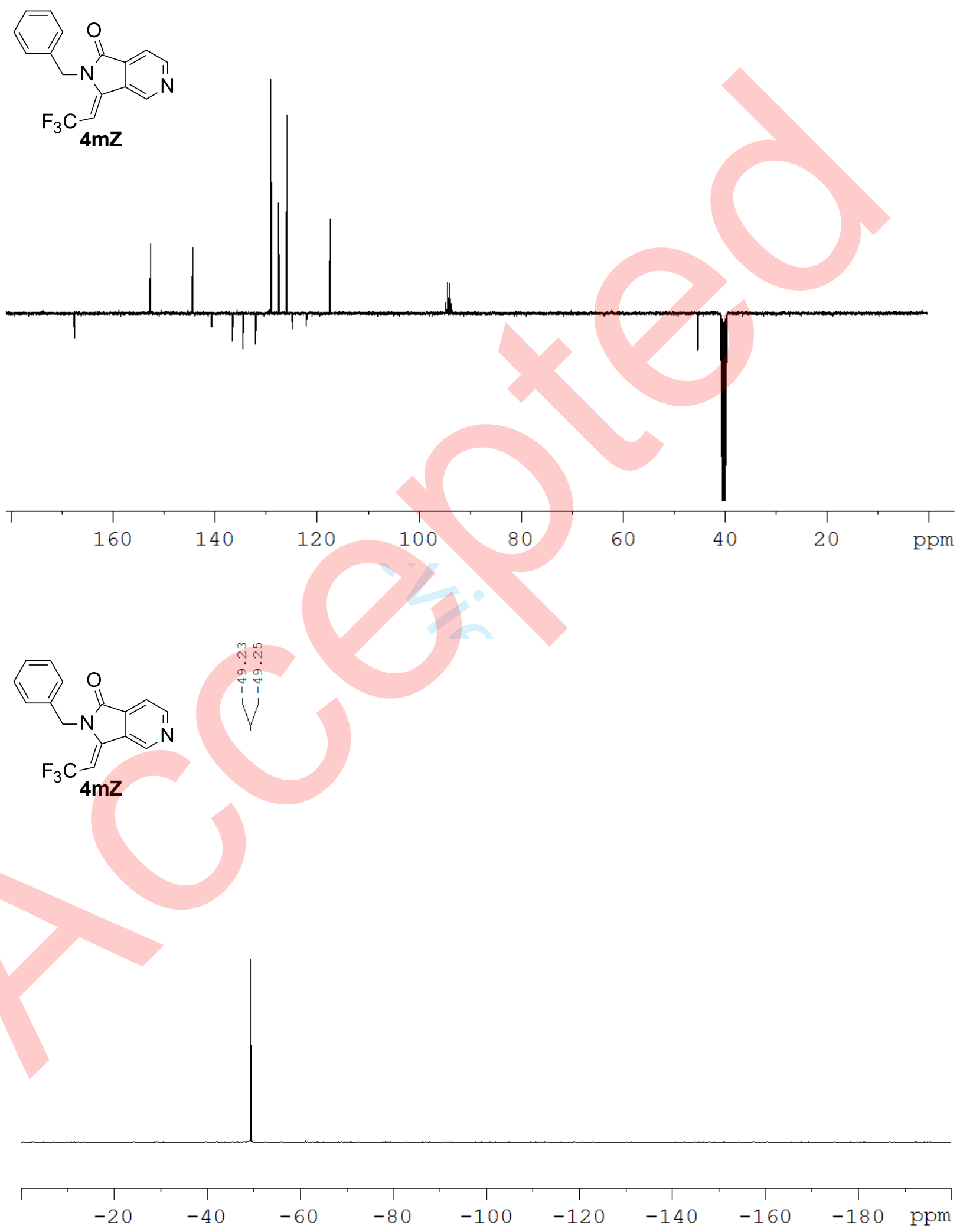


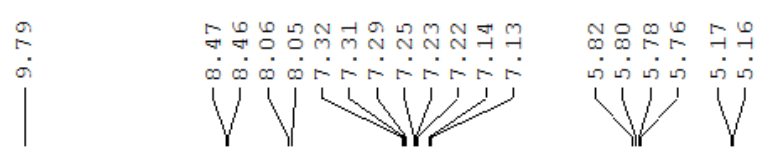<smiles>O=C1c2c(ccc3ncsc23)C(=CC(F)(F)F)N1Cc1ccccc1</smiles>

$4 n E$ II $4 \mathrm{nZ}$
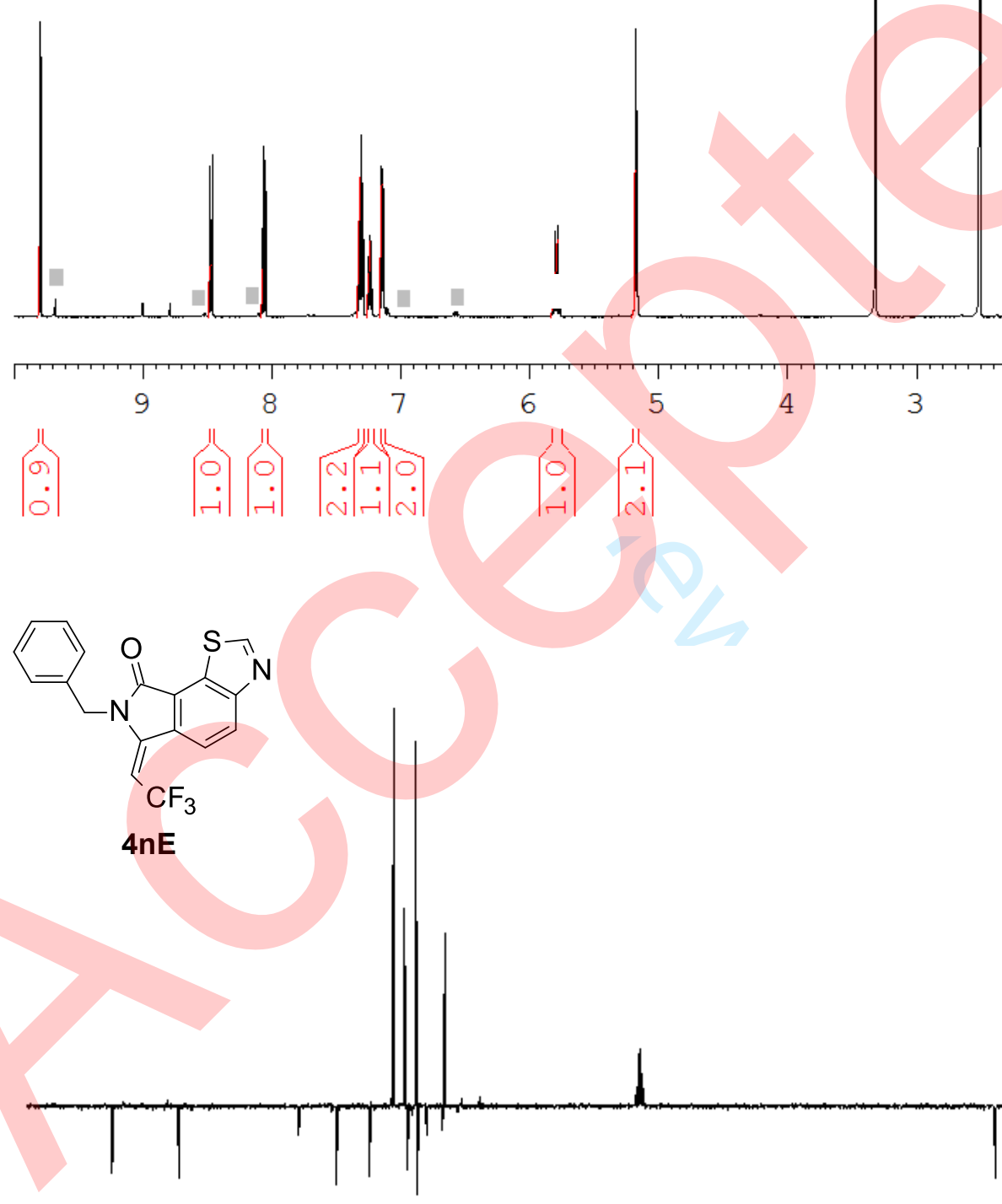

50 
Page 123 of 133

Science China Chemistry

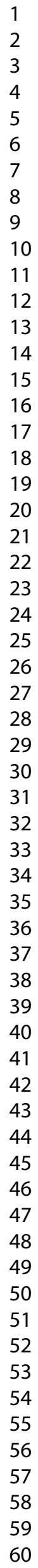<smiles>O=C1c2c(ccc3ncsc23)/C(=C\C(F)(F)F)N1Cc1ccccc1</smiles>

$\begin{array}{cc}0 \\ \infty & \infty \\ \infty & \infty \\ 0 & 0 \\ 7 & 1 \\ 1 & 1 \\ 1\end{array}$

$4 n Z$
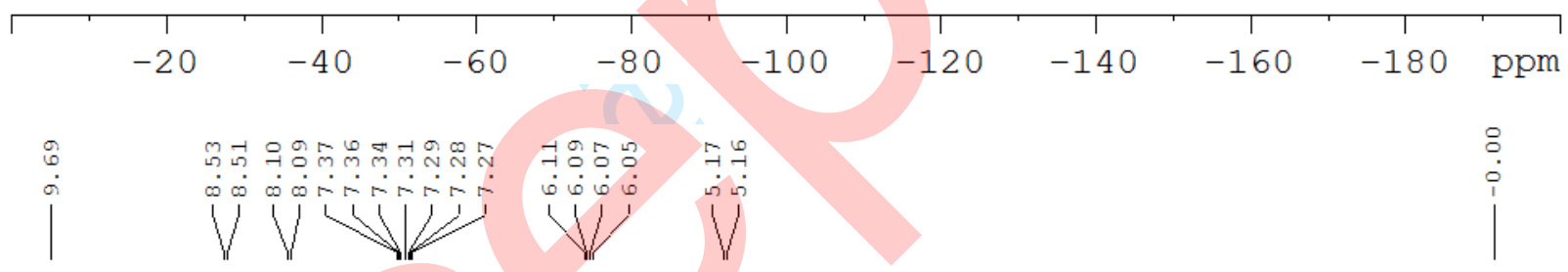

8
$\vdots$
1

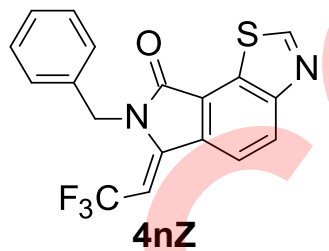

$4 \mathrm{nE}$
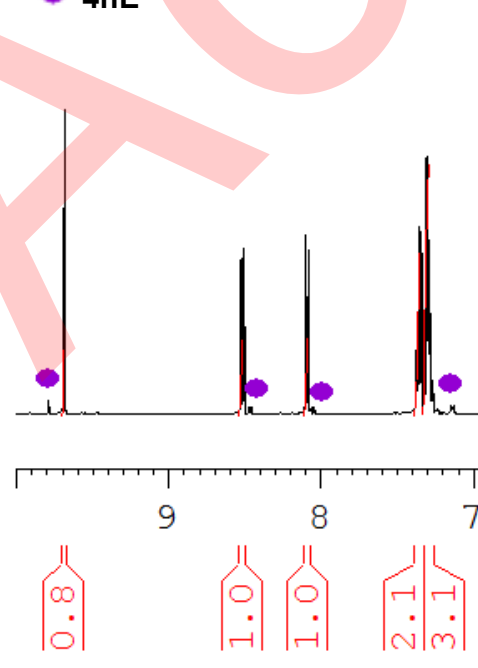

7

6

10

4

$\left|\begin{array}{l}0 \\ 0\end{array}\right|$

116

Downloaded to IP: 192.168.0.213 On: 2019-09-26 03:21:34 http://engine.scichina.com/doi/10.1007/s11426-019-9627-x http://chem.scichina.com/english 

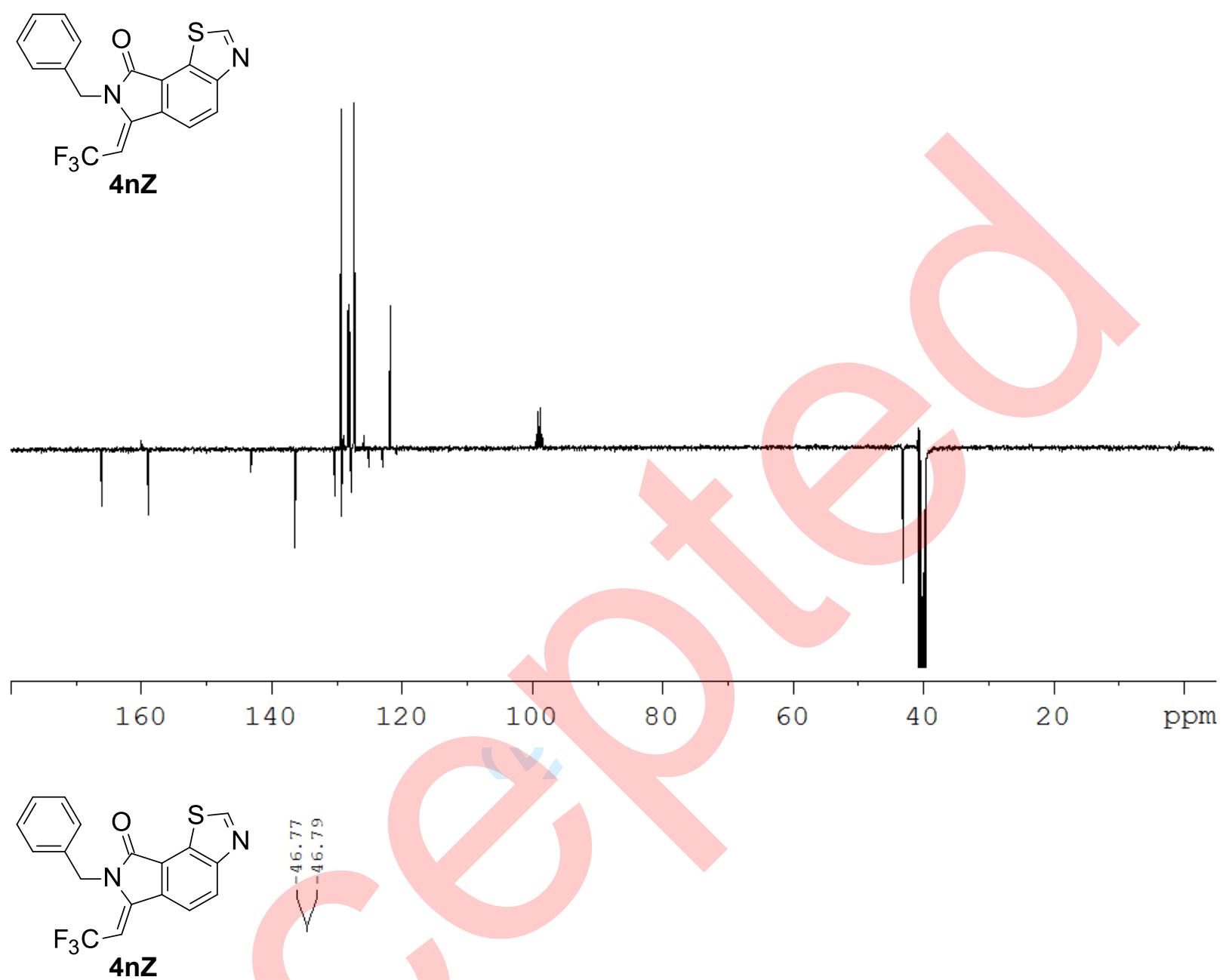

- 4nE 


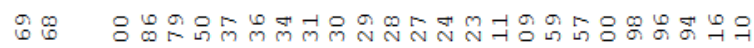

कं

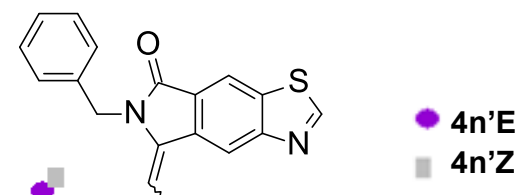

$4 n^{\prime} \mathrm{CF}_{3}$

$4 n ' Z$
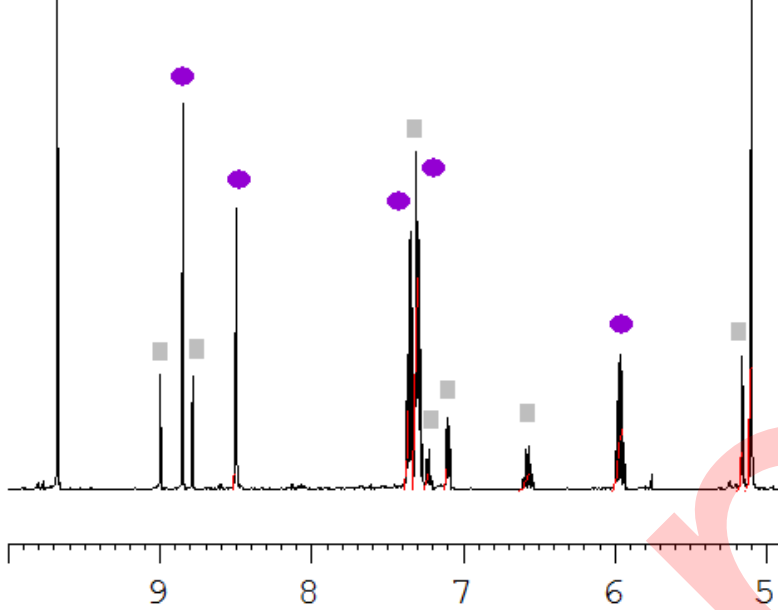

8

7

6

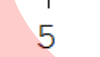

4

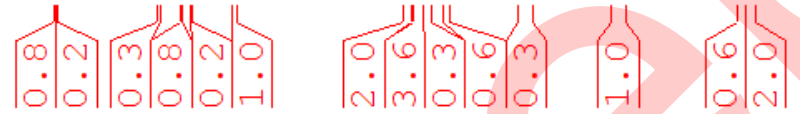

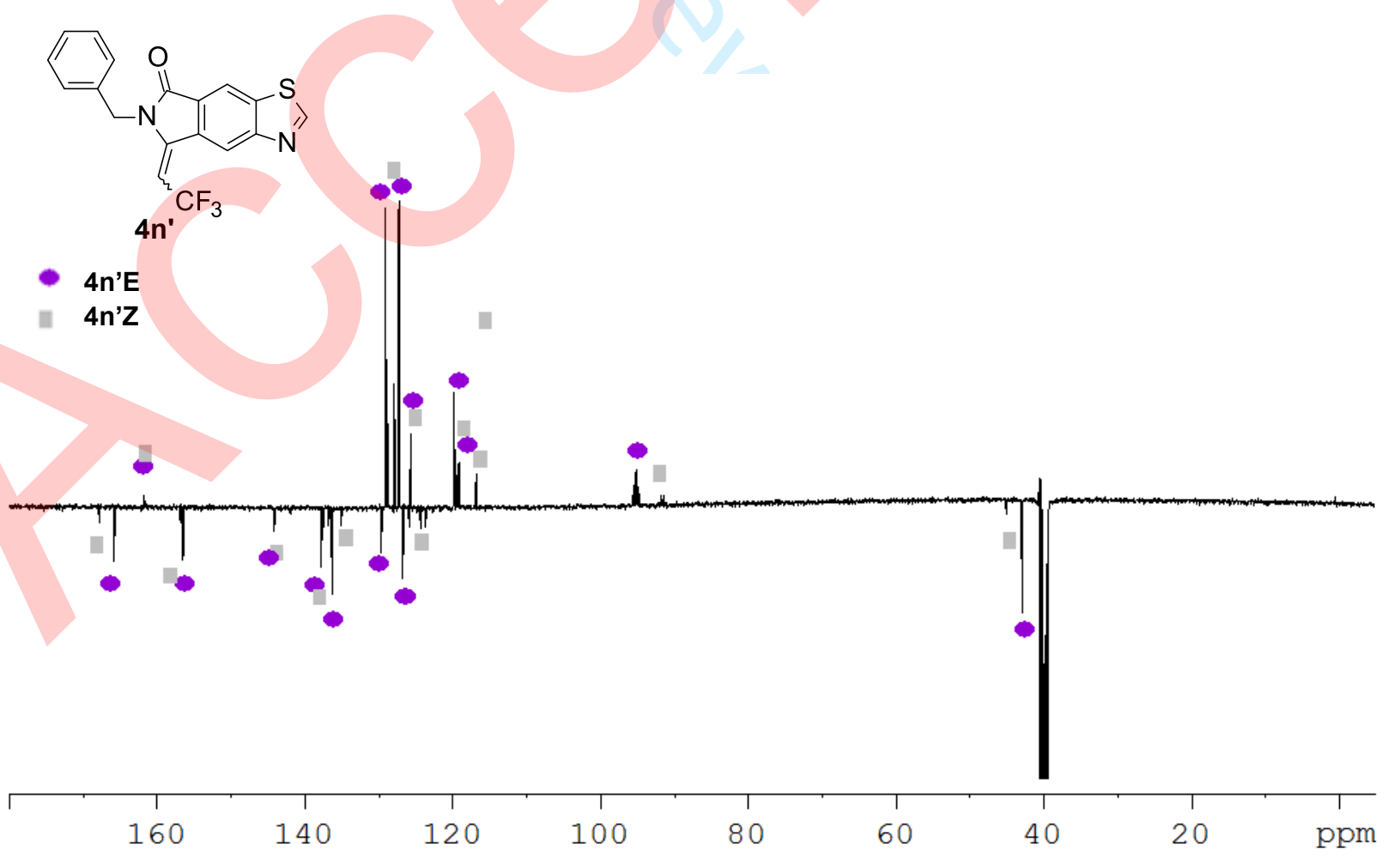




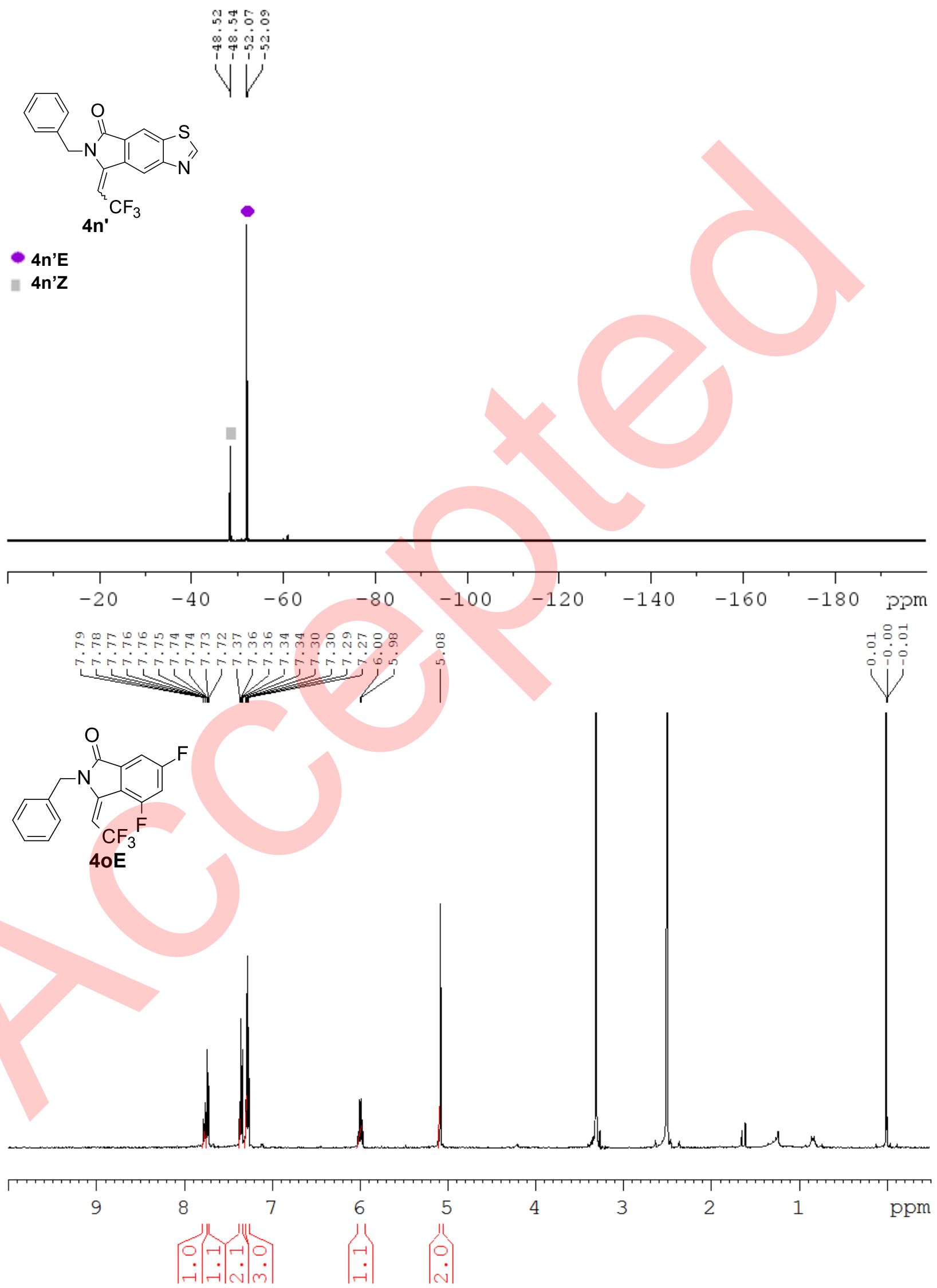



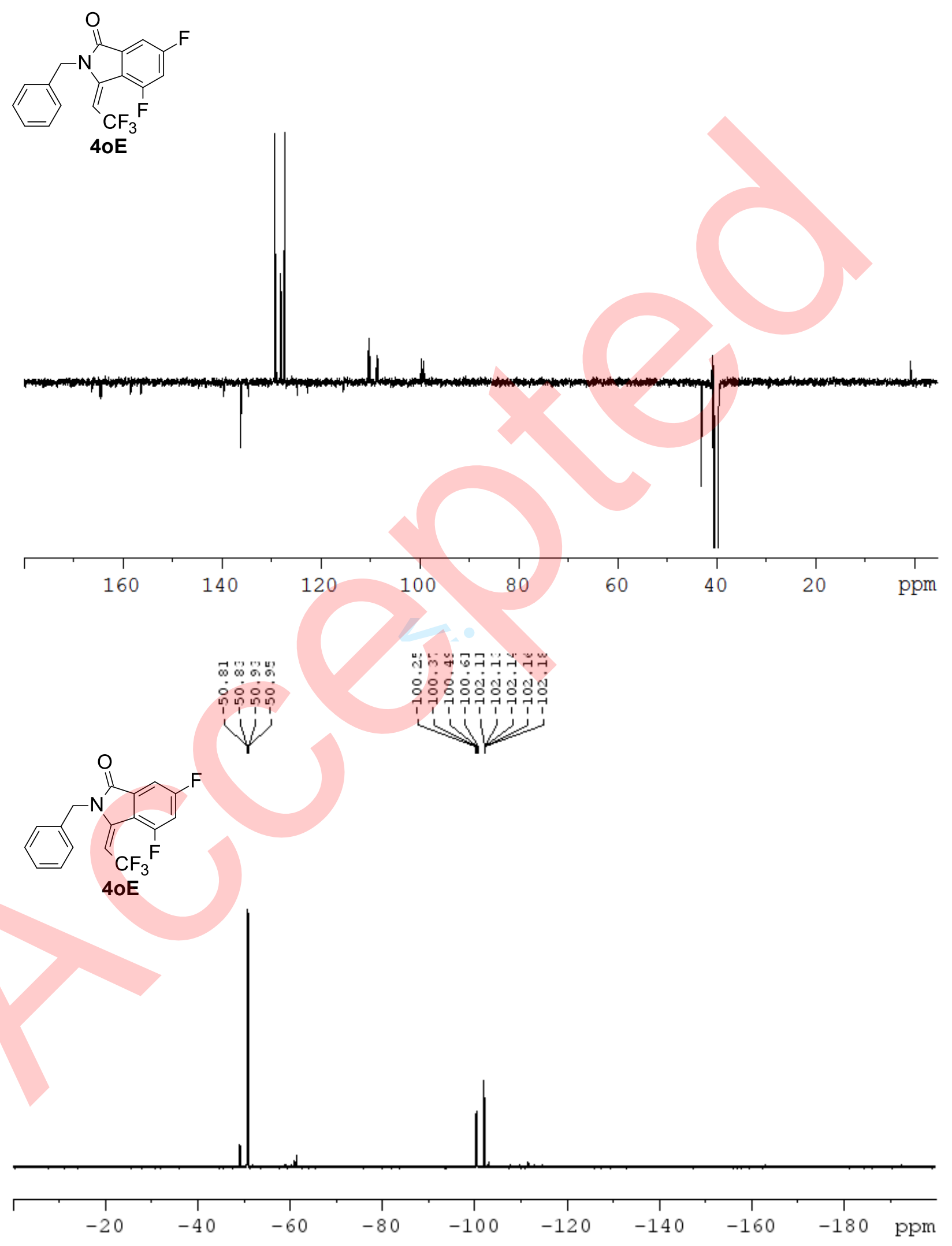


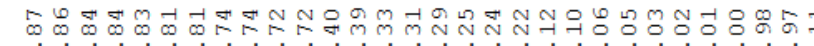

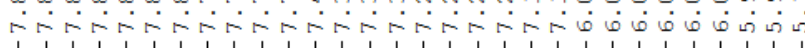<smiles>O=C(O)C=C1c2c(F)cc(F)cc2C(=O)N1Cc1ccccc1</smiles>

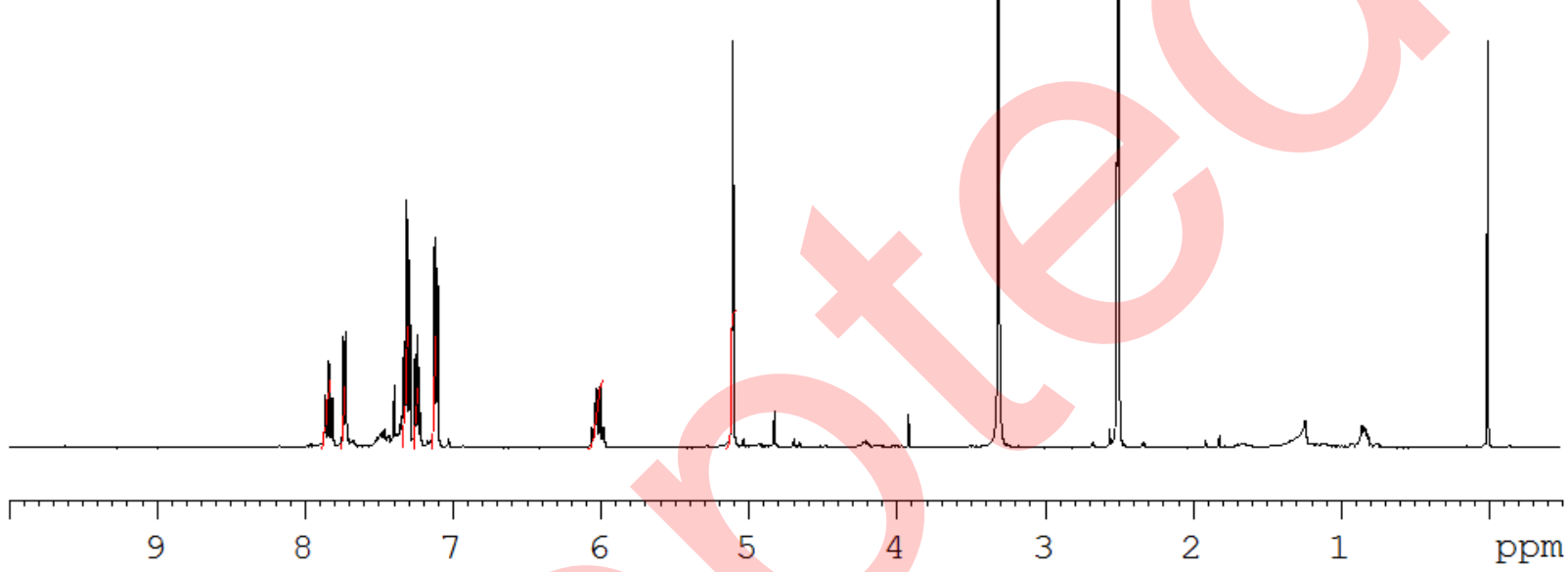

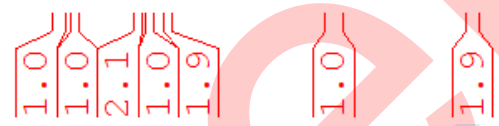
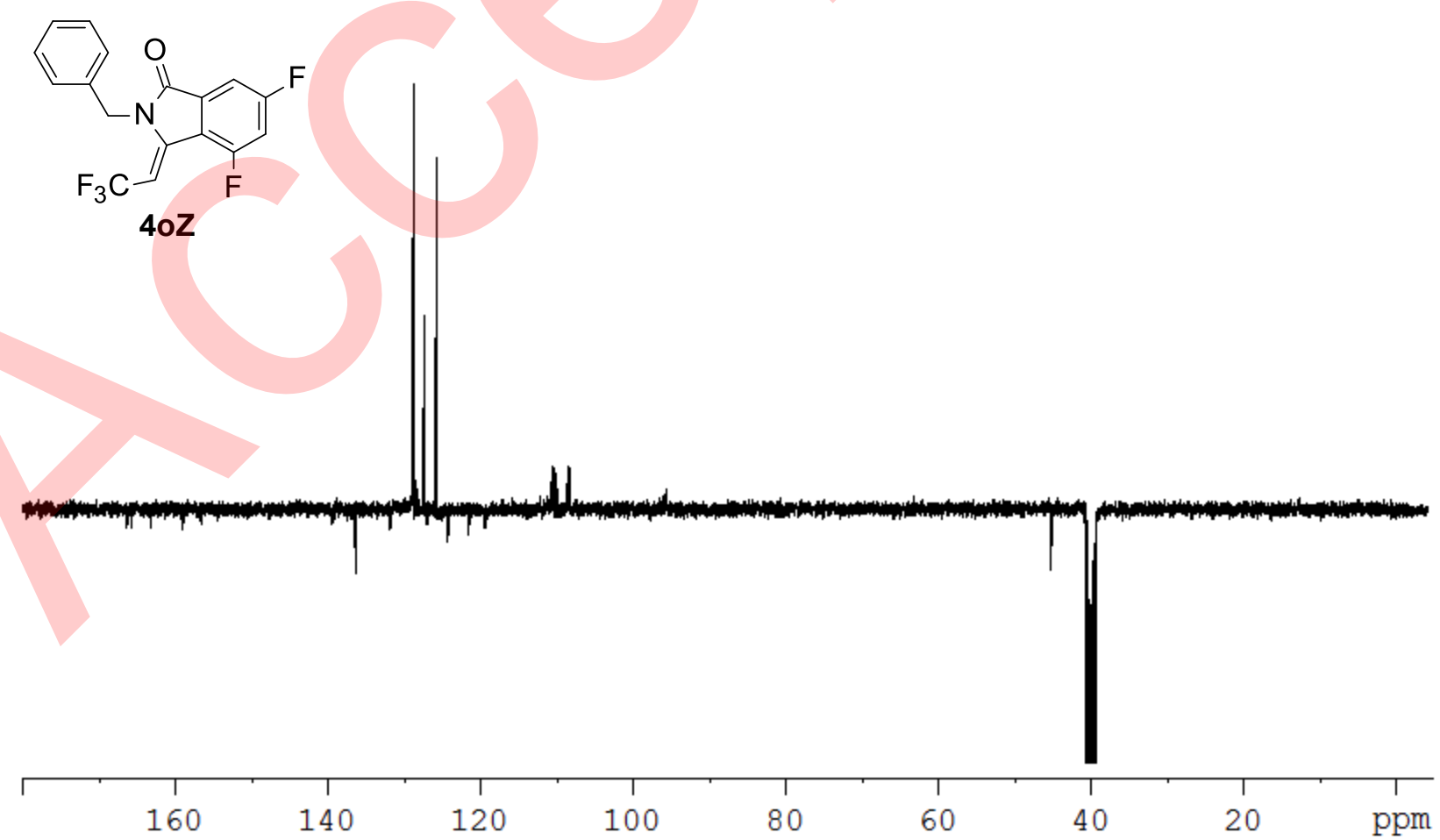

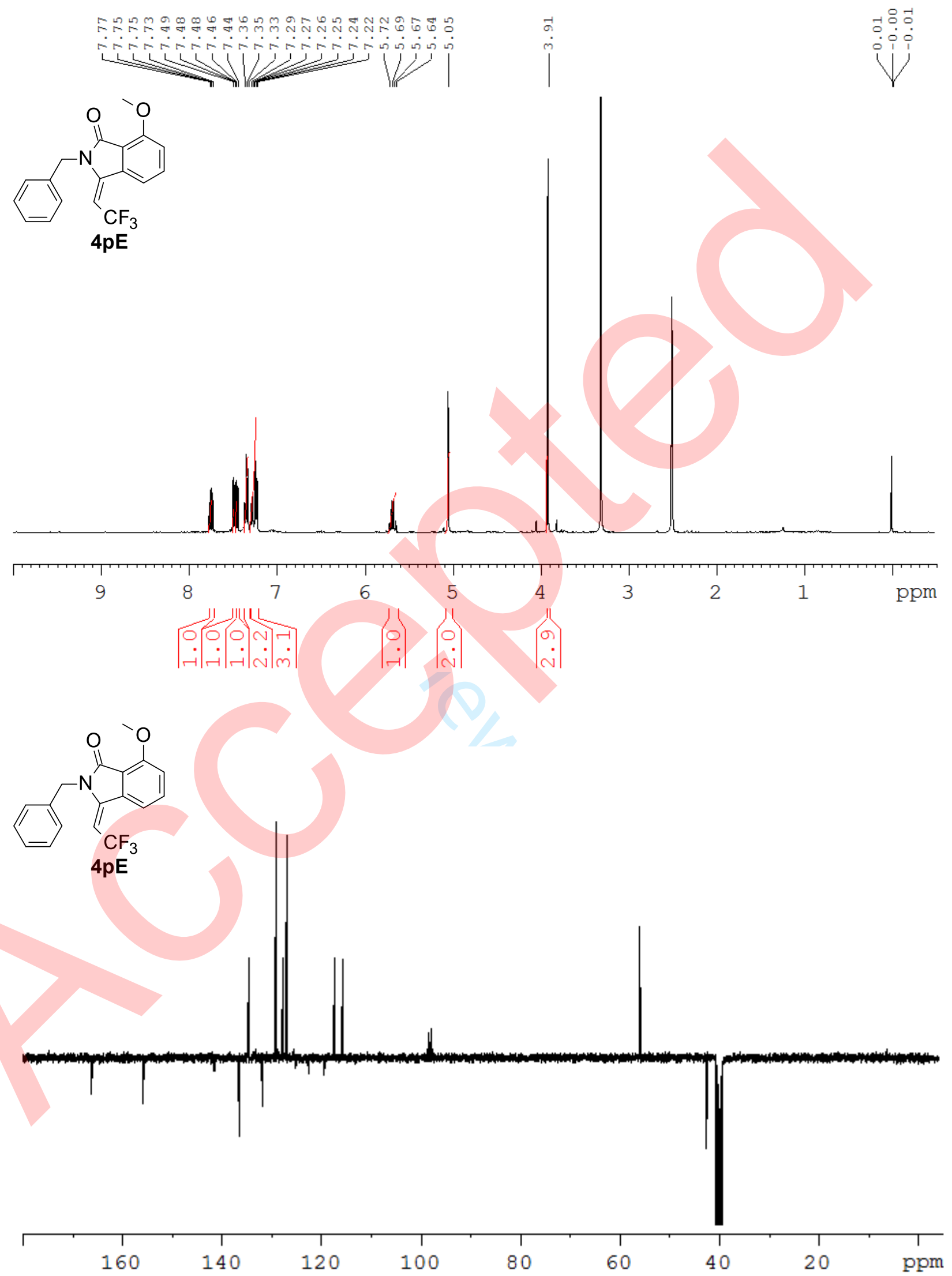

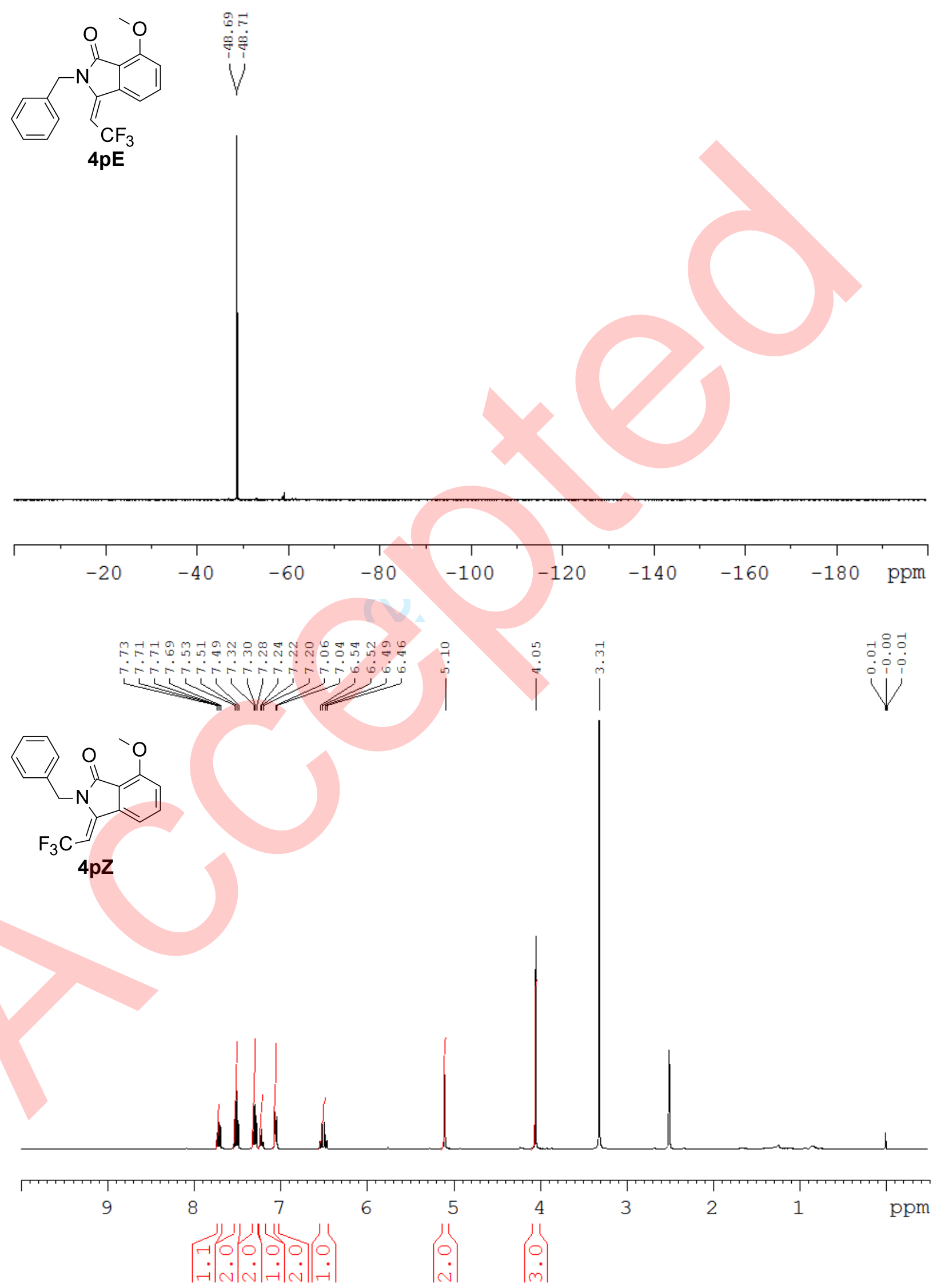

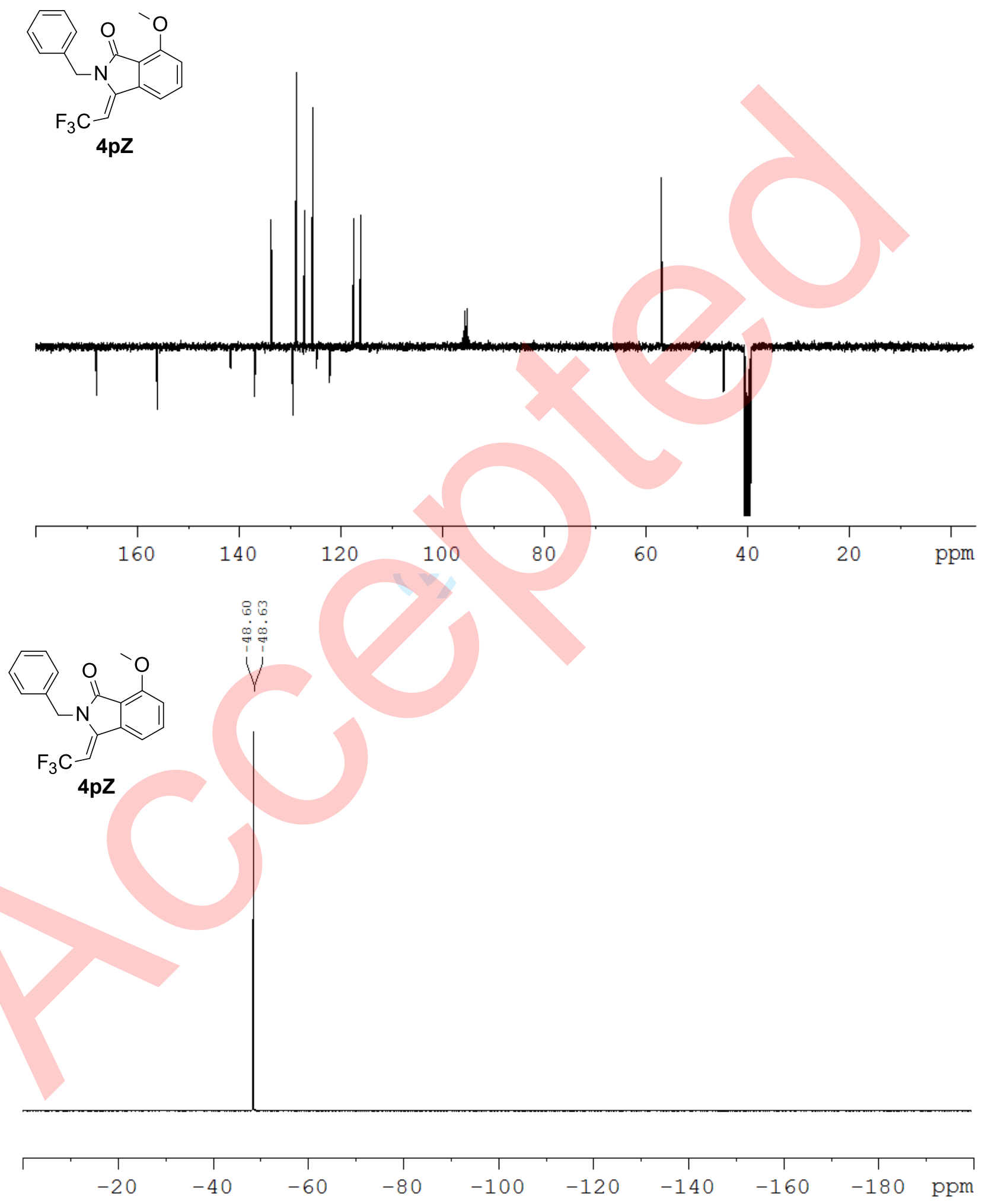
<smiles>COCCCN1C(=O)c2ccccc2/C1=C\C(F)(F)C(F)(F)F</smiles>

- 4qE

II $4 q Z$
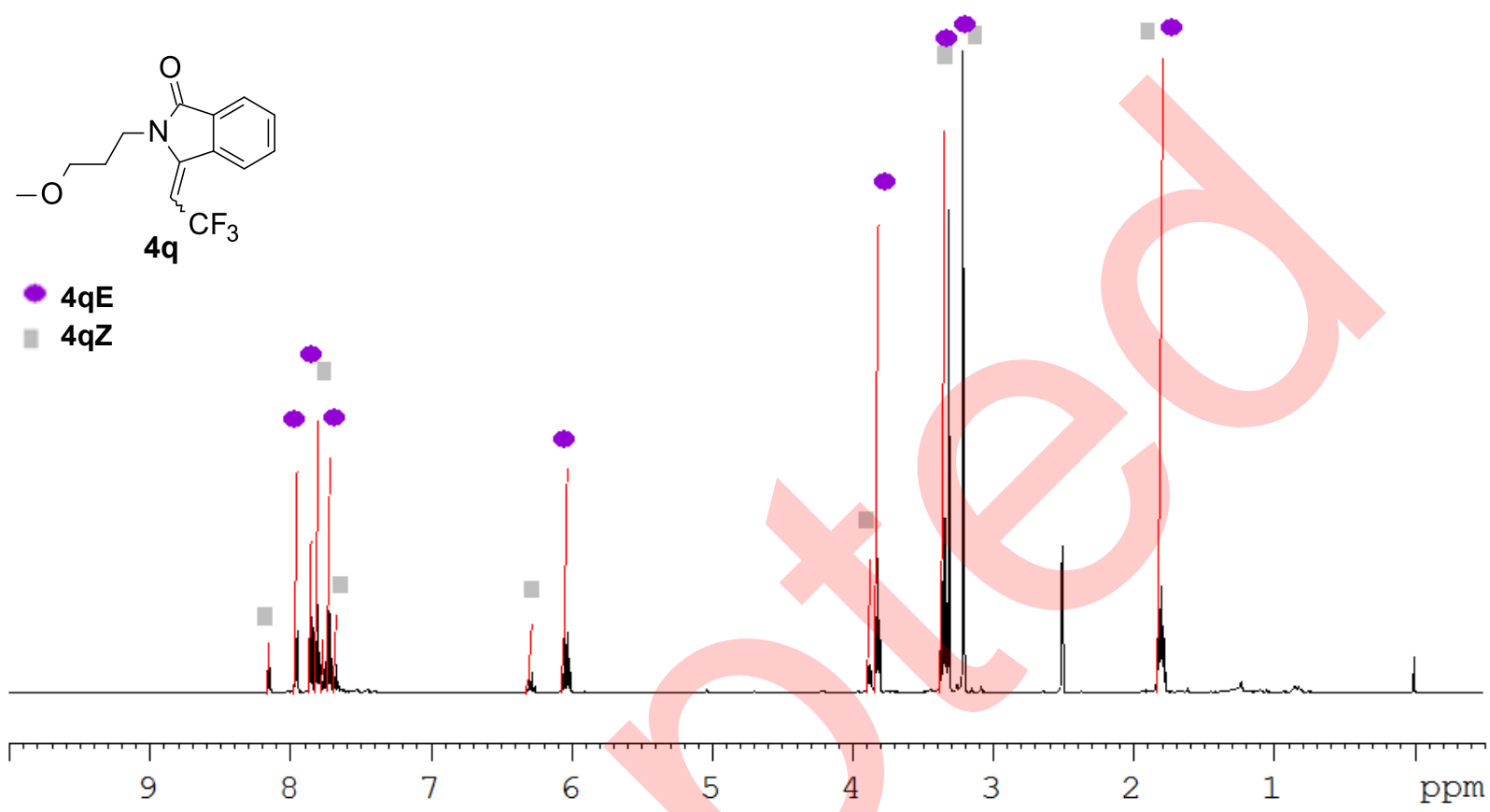

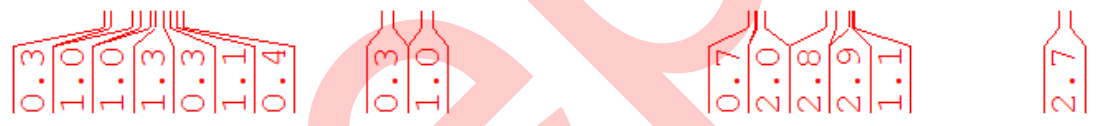
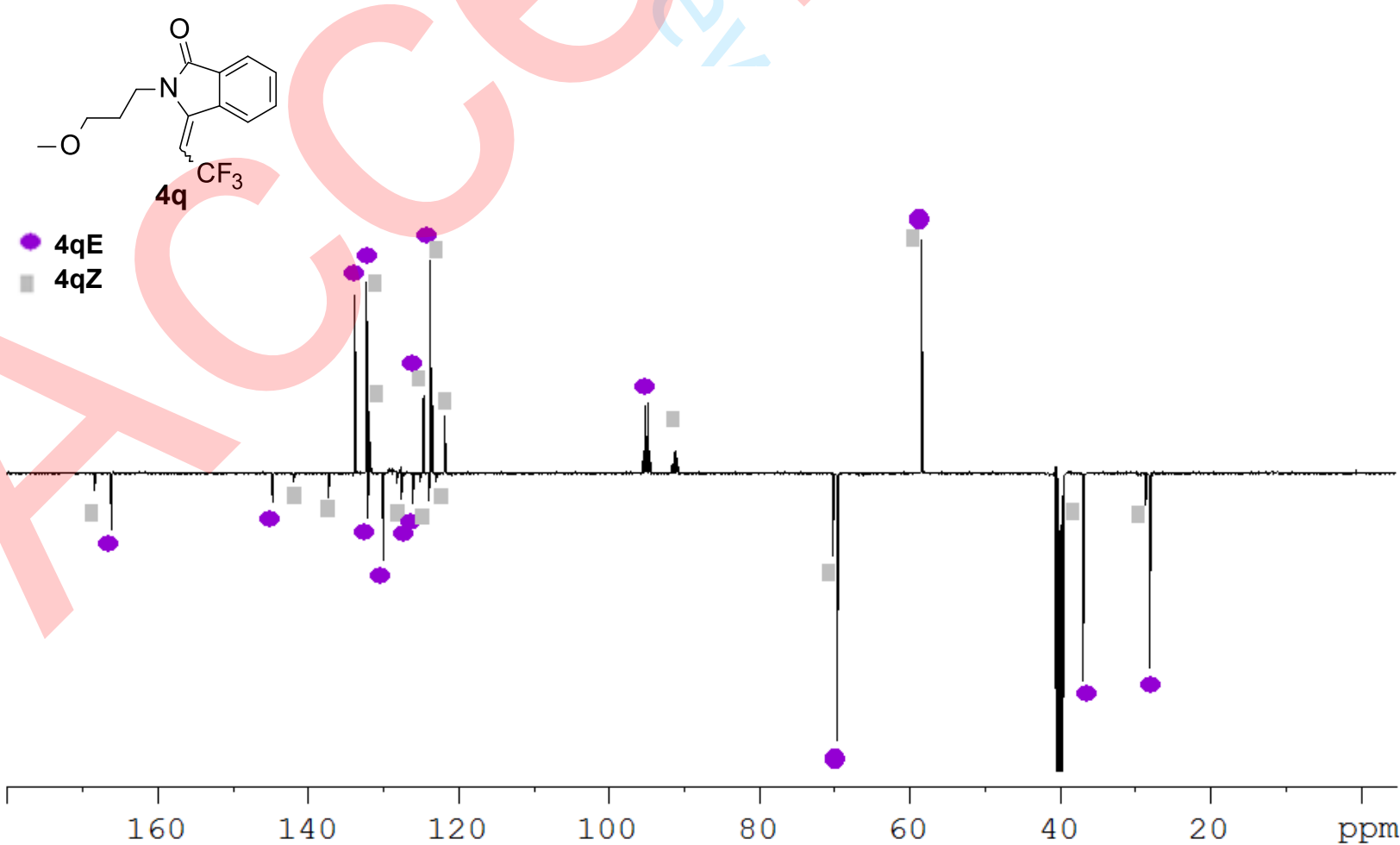


$$
\mathrm{c}^{\mathrm{c}^{\mathrm{e}}}
$$

